University of Tennessee Health Science Center UTHSC Digital Commons

\title{
$5-2006$
}

\section{Genetic Networks Modulating Retinal Injury}

Felix Vazquez-Chona

University of Tennessee Health Science Center

Follow this and additional works at: https://dc.uthsc.edu/dissertations

Part of the Genetic Processes Commons

\section{Recommended Citation}

Vazquez-Chona, Felix, "Genetic Networks Modulating Retinal Injury" (2006). Theses and Dissertations (ETD). Paper 286. http://dx.doi.org/10.21007/etd.cghs.2006.0337.

This Dissertation is brought to you for free and open access by the College of Graduate Health Sciences at UTHSC Digital Commons. It has been accepted for inclusion in Theses and Dissertations (ETD) by an authorized administrator of UTHSC Digital Commons. For more information, please contact jwelch30@uthsc.edu. 


\title{
Genetic Networks Modulating Retinal Injury
}

\begin{abstract}
Many advances have and are being made with the advent of modern sciences. One area that remains resistant to significant advances is the recovery of the mammalian central nervous system (CNS) after injury. Considerable efforts were placed on understanding the cellular and biochemical changes occurring after injury and during wound healing. Aside from cataloging the changes that occur, little progress is being made in understanding the molecular cascades associated with the control of the healing process. The present series of studies define the global changes that occur after injury. They also define CNS repair mechanisms by identifying genetic networks that underlie the temporal changes of retinal wound healing. Gene expression changes in the injured rat retina were analyzed with microarray technology, genetic linkage analysis of gene expression, and higher-level bioinformatic analyses. This work yielded three complementary insights. First, groups of functionally related genes underlie the early, delayed, and sustained responses of wound healing. For example, transcriptional factors such as Fos and Egr1 define the early response, whereas, glial reactive markers such as Gfap and Cd81 define the sustained response. Second, three specific genomic loci modulate coordinated changes in gene expression in mouse brains: regulatory loci on chromosomes 6,12 , and 14 . Of the three only the regulatory locus on chromosome 12 specifically modulates the expression of a group of genes involved in early wound-healing events (mainly, the regulation of transcription, differentiation, apoptosis, and proliferation). Third, candidate genes Id2 and Lpin 1 are current modulators for the network controlled by the chromosome 12 locus. Higher levels of Id2 and Lpin1 correlated with higher levels of the survival gene Crygd, and with lower levels of acute phase genes Fos and Stat3, reactive gliosis genes Gfap and Cd81, and apoptotic gene Casp3. In this body of work I have moved beyond cataloging changes in the transcriptome to identifying candidate genes modulating the retinal response to injury. During this process I developed an integrated approach of gene expression profiling and higher-level bioinformatic analyses to define genetic networks. This work not only advances our understanding of the molecular networks controlling the CNS response to injury, but may also form the basis for interventions that can rescue injured neurons and re-establish lost CNS connections.
\end{abstract}

\section{Document Type}

Dissertation

Degree Name

Doctor of Philosophy (PhD)

Program

Anatomy

Research Advisor

Eldone E. Geisert, Ph.D.

\section{Keywords}

retina, degeneration, regeneration, microarray, glia, gliosis, gene expression, gene regulation, quantitative trait locus (QTL) analysis, expression genetics, microarray

\section{Subject Categories}

Genetic Processes | Medical Sciences | Medicine and Health Sciences 


\title{
GENETIC NETWORKS
}

\section{MOdULATING RETINAL INJURY}

\author{
A Dissertation \\ Presented for \\ The Graduate Studies Council \\ The University of Tennessee \\ Health Science Center
}

In Partial Fulfillment

Of the Requirements for the Degree

Doctor of Philosophy

From The University of Tennessee

By

Félix Vázquez-Chona

May 2006 
Chapter $2 \square 2004$ by Association for Research in Vision and Ophthalmology

Chapter $3 \square 2005$ by Molecular Vision

All other material $\square 2006$ by Félix Vázquez-Chona 


\title{
Dedication
}

\author{
To my parents
}

\section{Rosa Carmina Chona-Hernandez \\ Feliciano Vázquez-Almaraz \\ Jenine R. Riddle \\ Herman R. Riddle}

and

To my wife

Sara R. Vázquez 


\section{Dissertation Abstract}

Many advances have and are being made with the advent of modern sciences. One area that remains resistant to significant advances is the recovery of the mammalian central nervous system (CNS) after injury. Considerable efforts were placed on understanding the cellular and biochemical changes occurring after injury and during wound healing. Aside from cataloging the changes that occur, little progress is being made in understanding the molecular cascades associated with the control of the healing process. The present series of studies define the global changes that occur after injury. They also define CNS repair mechanisms by identifying genetic networks that underlie the temporal changes of retinal wound healing. Gene expression changes in the injured rat retina were analyzed with microarray technology, genetic linkage analysis of gene expression, and higher-level bioinformatic analyses. This work yielded three complementary insights. First, groups of functionally related genes underlie the early, delayed, and sustained responses of wound healing. For example, transcriptional factors such as Fos and Egrl define the early response, whereas, glial reactive markers such as Gfap and Cd81 define the sustained response. Second, three specific genomic loci modulate coordinated changes in gene expression in mouse brains: regulatory loci on chromosomes 6,12 , and 14 . Of the three only the regulatory locus on chromosome 12 specifically modulates the expression of a group of genes involved in early wound-healing events (mainly, the regulation of transcription, differentiation, apoptosis, and proliferation). Third, candidate genes Id2 and Lpin1 are current modulators for the network controlled by the chromosome 12 locus. Higher levels of Id 2 and Lpinl correlated with higher levels of the survival gene Crygd, and with lower levels of acute phase genes Fos and Stat3, reactive gliosis genes Gfap and Cd81, and apoptotic gene Casp3. In this body of work I have moved beyond cataloging changes in the transcriptome to identifying candidate genes modulating the retinal response to injury. During this process I developed an integrated approach of gene expression profiling and higher-level bioinformatic analyses to define genetic networks. This work not only advances our understanding of the molecular networks controlling the CNS response to injury, 
but may also form the basis for interventions that can rescue injured neurons and re-establish lost CNS connections. 


\section{Table of Contents}

Chapter 1. Introduction 1

Retinal Wound Healing $\quad 2$

Gene Expression Profiling $\quad 8$

Genetics of Gene Expression $\quad 9$

Aims of the Dissertation 12

Chapter 2. Temporal Changes in Gene Expression After Injury in the Rat Retina 14

$\begin{array}{ll}\text { Abstract } & 15\end{array}$

$\begin{array}{ll}\text { Introduction } & 16\end{array}$

$\begin{array}{ll}\text { Methods } & 17\end{array}$

$\begin{array}{ll}\text { Results } & 21\end{array}$

$\begin{array}{ll}\text { Discussion } & 34\end{array}$

Chapter 3. Genetic Networks Controlling Retinal Injury 39

$\begin{array}{ll}\text { Abstract } & 40\end{array}$

$\begin{array}{ll}\text { Introduction } & 41\end{array}$

$\begin{array}{ll}\text { Methods } & 43\end{array}$

$\begin{array}{ll}\text { Results } & 48\end{array}$

$\begin{array}{ll}\text { Discussion } & 63\end{array}$

Chapter 4. Genetic Influences on Gene Expression and Retinal Wound Healing 69

$\begin{array}{ll}\text { Introduction } & 70\end{array}$

$\begin{array}{ll}\text { Methods } & 72\end{array}$

$\begin{array}{ll}\text { Results } & 79\end{array}$

$\begin{array}{ll}\text { Discussion } & 97\end{array}$

$\begin{array}{ll}\text { Chapter 5. Discussion } & 103\end{array}$

Cataloging Global Changes During Wound Healing Using Microarrays 104

Defining Regulatory Loci That Modulate Gene Expression 110 
Finding Regulators of Retinal Wound Healing

Conclusion

Appendix A. Minimum Information About a Microarray Experiment (MIAME) Report

Appendix B. Supplemental Material for Chapter 2

Appendix C. Supplemental Material for Chapter 4 


\section{List of Tables}

2.1. Experiments and distribution of animals 18

2.2. Microarray expression profiles after an experimentally induced retinal tear 25

2.3. Transcript changes confirmed with real-time RT-PCR 26

3.1. Data used to define acute phase genes $\quad 44$

3.2. Acute phase genes expressed in the injured CNS 49

4.1. Oligonucleotides used for RNA interference and real-time RT-PCR 77

B.1. Experimental conditions, samples, and GEO accession numbers 142

B.2. Oligonucleotides used for real-time RT-PCR 143

B.3. Correlation between normalized MAS 5.0 and RMA signals 145

B.4. Gene clusters in the injured retina 146

B.5. Gene functional clusters in injured retina $\quad 150$

C.1. Wound-healing genes modulated by chromosome 12 locus 154 


\section{List of Figures}

1.1. Cellular response to retinal injury $\quad 4$

2.1. The cellular response of the rat retina to injury 22

2.2. Gene expression profiles after retinal injury 28

2.3. Temporal distribution of genes and functional categories after retinal injury 30

2.4. The crystallins $\square, \square$, and $\square$ were dramatically upregulated after retinal injury 31

2.5. $C d 81$ clustered into the late-response profile along with tetraspanins and glial reactive $\begin{array}{ll}\text { markers } & 33\end{array}$

3.1. Acute phase genes share regulatory loci $\quad 51$

3.2. Genetic networks controlling acute phase transcripts 53

3.3. Evaluating candidate genes within chromosome 14 locus 55

3.4. Evaluating candidate genes within chromosome 6 locus 58

3.5. Evaluating candidate genes within chromosome 12 locus 61

4.1. Chromosome 12 locus modulates wound-healing mechanisms in the CNS 80

4.2. Chromosome 12 locus modulates transcription, differentiation, proliferation, and $\begin{array}{ll}\text { apoptotic mechanisms } & 84\end{array}$

4.3. Candidate genes for chromosome 12 network 86

4.4. Id2 and Lpin1 are differentially expressed in response to genetic differences and trauma 91

4.5. Higher levels of Id2 and Lpinl correlated with lower retinal stress markers 93

4.6. Id2 silencing decreases the mitotic activity and healing response of cultured astrocytes 96

5.1. Gene expression after injury is temporally regulated and gene product functions are consistent with known the biology of retinal wound healing 108

5.2. Locus on chromosome 12 modulates the expression of genes involved in differentiation, $\begin{array}{ll}\text { proliferation, and cell death } & 111\end{array}$

5.3. Id2 is a candidate gene modulating the chromosome 12 network 117

5.4. Model of Muller cell activation by chromosome 12 network 121 
B.1. Effect of expression level on coefficient of variation

C.1. Expression patterns for chromosome 12 network genes during retinal development and retinal injury

D.1. ID2 antibody control using retina from $I d 2$-null mice 
Chapter 1

Introduction 


\section{Retinal Wound Healing}

The retina responds to injury and chronic stress by activating a complex series of cellular events that collectively preserve the structure and function of the retina. This wound-healing response can result in permanent cellular changes that can lead to secondary complications and the loss of visual function. This protective mechanism may be a significant barrier to therapeutic measures designed to preserve visual function such as cell transplantation, gene therapy, and bioinic implants. Defining the molecular changes that underlie the retinal healing is key to finding pathways that can be targeted to modulate the complications of retinal repair.

The retina is a complex tissue made of different cell types segregated into distinct cellular layers with associated functional properties. The transduction of light to a neural signal occurs at the back of the retina within the photoreceptors. The signal is processed by bipolar, amacrine and horizontal cells. The signal is relayed to the brain by the retinal ganglion cells. The sensory and processing functions of the neural retina are guarded by the supporting glial cells. The two major retinal glial cells are the Muller cells and the retinal pigment epithelium (RPE). Retinal glial cells maintain homeostasis and the blood-retinal barriers. Muller cells extend radially spanning nearly the entire thickness of the neural retina from the internal limiting membrane to the external limiting membrane. As these cells span the retina they interact with virtually all the retinal cell types [Sarthy and Ripps, 2001]. RPE cells lie at the back of the eye between the neural retina and the choroid. This location allows the RPE to closely associate with the outer segments of photoreceptors in addition to serving as a barrier protecting the neural retina from the choroidal circulation [Zhao et al., 1997]. Together, Muller and RPE cells are well positioned to be exposed to degenerative changes in neural cells and to play a key role in the wound healing response.

\section{Wound-Healing Animal Models}

Studies in animal models have provided information about the cellular response to retinal injury. Stephen J. Ryan's group described the histological features of the inflammatory response to a 
penetrating injury that disrupts the sclera and the retina [Gregor and Ryan, 1986; Cleary and Ryan, 1979]. Others have described the neuronal and glial response after retinal breaks induced with scrapes [Miller and Oberdorfer, 1981; Maclaren, 1996] and laser photocoagulation lesions [Humphrey et al., 1993]. Steven K Fisher's group examined the cellular response to the separation of the neural retina from the RPE layer in a model of retinal detachment in cats [Anderson et al., 1983]. The breakdown of the blood-retinal barrier (BRB) using non-invasive mechanism was produced by Peter A. Campochiaro's group using intravenous sodium iodate or intravitreal injection of epinephrine [Campochiaro et al., 1986]. The response of glial cells was also studied when specific neuronal cells were damaged. Optic nerve crushes target the cell death of retinal ganglion cells which is preceded by the activation of retinal glia [Bahr, 1991]. Targeted cell death of photoreceptors in albino animals by fluorescent light also activates retinal glia. As a general survey of the retinal response to injury, this introduction describes the wound healing response to a mechanical disruption of the retina. The retinal glial cells undergo temporal and spatial changes that can be divided into three major phases: an early acute phase (minutes, hours), a delayed subacute phase (days) and late chronic phase (days, weeks) (Figure 1.1).

\section{Wound Healing Phases}

The immediate consequences of trauma include hemorrhage, the formation of a toxic environment, and the activation of glial cells. Direct mechanical trauma to the retina interrupts the blood-retinal barriers, intrudes on the vitreous, and disrupts the subretinal contents into the neural retina [Postel and Mieler, 1999; Gregor and Ryan, 1982]. The flow of fluid and metabolites into the retinal microenvironment creates a highly acidic and toxic environment [Liang and Godley, 2003; Sherry and Townes-Anderson, 2000]. The initial glial responses to injury are simply normal glial properties operating in an abruptly altered environment. For example, the Muller cells attempt to restore ionic and glutamate balance of the extracellular environment; and, RPE cells phagocytize debris and fragmented cells [Bringmann et al., 2000; Sherry and Townes-Anderson, 2000; Zhao et al., 1997]. The increased physiological demands on 


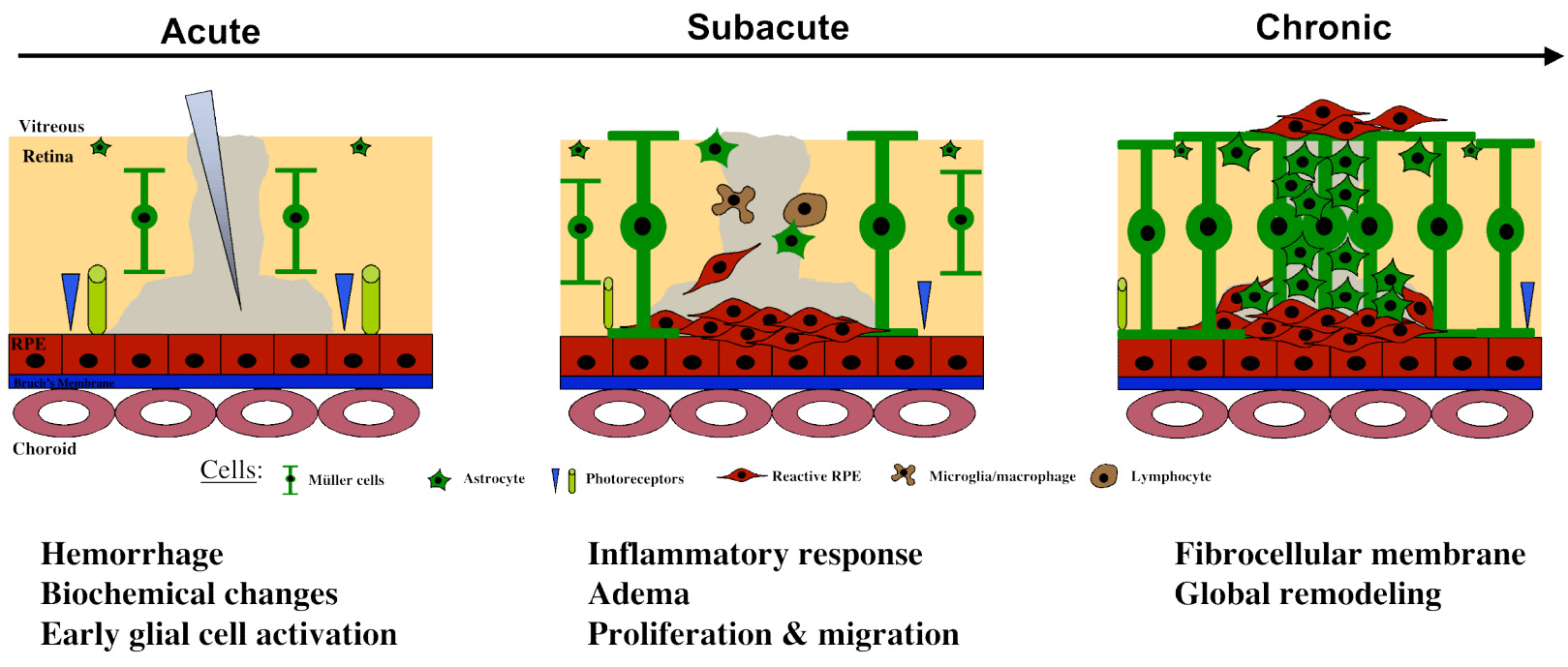

Figure 1.1.Cellular response to retinal injury. This schematic describes the wound healing response to a mechanical disruption of the retina (for example, a retinal tear). The retinal glial cells undergo temporal and spatial changes that can be divided into three major phases: an early acute phase (minutes, hours), a delayed subacute phase (days) and late chronic phase (days, weeks). 
glial cells result in the swelling of glial cells. Serum factors released from the blood stream and vitreous material are known to activate the release of pro-inflammatory cytokines from glia cells, and activate the early activation of signaling cascades involved in proliferation and survival [Geller et al., 2001; Takeda et al., 2002; Izumi et al., 2002]

The subacute phase is characterized by inflammatory responses and phenotypic changes in glial cells. The early release of cytokines by damaged cells and activated glial cells recruit inflammatory cells from the blood stream to the site of injury [Zhou et al., 2005; Zhou and Maren, 2003; Szabo et al., 1991]. Blood derived neutrophils and macrophages as well as endogenous microglia infiltrate the lesion as early as day 1 after injury and peak at day 3 [Zhou et al., 2005; Zhou and Maren, 2003]. Together the inflammatory cells clear fragmented cells and foreign material, and release additional inflammatory factors (cytokines, chemokines, and nitric oxide synthetase). The peak of inflammatory factors prolongs the wave of inflammatory cells and results in edema [Limb et al., 1991; Fisher et al., 1991]. The inflammation and edema can cause further breakdown of the blood-retinal barrier [Vinores et al., 2001; Behar-Cohen et al., 2000].

The loss of retinal cells and the exposure to components of the blood and the vitreous trigger a reactive state in glial cells that includes changes in cell shape, molecular content, and cellular behavior. The loss of retinal cells caused by the initial trauma and cell death activates morphological changes in glial cells. For example, Muller cells enlarge their cell body and extend their processes into the area of cell loss [Lewis et al, 2003; Marc et al., 2003]. RPE cells change shape from cuboidal to fibroblas-like and macrophage-like [Grierson et al., 1994]. One of the defining characteristics in the activation of glial cells in the retina is a reorganization of cytoskeletal content. The most obvious change is an elevation in intermediate filaments. For Muller cells the levels of glial fibrillary acidic protein (GFAP) and vimentin increase dramatically. In response to injury the RPE cells upregulate cytokeratins and vimentin [Lewis and Fisher, 2003; Stroeva and Mitashov, 1983; Zhao et al., 1997].

Glial cells can also migrate and proliferate. Migration can be activated by vitreal and blood components [Vidaurri-Leal et al., 1984; Gregor and Ryan, 1982; Campochiaro et al., 1986; 
Kim and Arroyo, 2002]. The collagen from the vitreous and the fibronectin from serum provide a rich substrate for migration. In addition, cytokines (specifically platelet-derived growth factor and transforming growth factor) released from the bloodstream and infiltrating cells are chemotactic and mitogenic for glial cells [Kim and Arroyo, 2002; Campochiaro et al., 1986; Vinores et al., 1992]. Blood contents also stimulate the proliferation of glial cells. Experimental models of retinal proliferation have demonstrated that hemoglobin and iron stimulate glial cell proliferation even in the absence of direct mechanical trauma to the retina [Burke and Smith, 1981]. Inflammation and cell proliferation resolve within the first week as the response to injury enters its chronic phase [Ryan et al., 1994; Fisher et al., 2001; Behar-Cohen et al., 2000].

During the late phase, glial cells remain reactive and participate in the local and global remodeling of the retina. Cells that migrate into the wound, vitreal space, and subretinal space transform the hemorrhage with fibrocellular membranes consisting of collagenous components and cellular components. With time, these membranes may contract, causing significant problems, including retinal detachment [Ryan et al., 1994; Fisher et al., 2001; Behar-Cohen et al., 2000; Grierson et al., 1994]. The formation of ectopic fibrocellular membranes in the retina is clinically referred as proliferative vitreoretinopathy [Chaum, 1995; Pastor et al., 2002]. There is also a change that occurs within the retina itself. Muller cells in the vicinity of the lesion activate expression of GFAP, extend their process, and increase in size. These changes continue in a late chronic activation of Muller cells that spreads distally to include eventually large extents of the retina [Lewis and Fisher, 2003; Bignami and Dahl, 1979; Humphrey 1993; Song et al., 2003; Berhar-Cohen et al., 2000; MacLaren, 1996; Miller and Oberdorfer, 1981]. Histologically this is seen by a sustained reactivity of GFAP in the cell bodies and process of Muller cells. The global upregulation of GFAP and hypertrophy in Muller cells are a common end point of the retinal response to various forms of injury including tears, toxic injuries and optic nerve damage. In addition to the cellular remodeling of glial cells display, neural cells neural cells activate a series of signaling pathways that culminate in neuronal migration, elaboration of new neurites and synapses, and rewiring of retinal circuits [Marc et al., 2003; Fisher et al., 2005; Jones et al., 
2003]. These structural changes are long lasting and represent at the present irreversible changes [Marc et al., 2003; Fisher et al., 2005; Grierson et al., 1994].

\section{Consequences of Retinal Healing and Remodeling}

As with many biological processes the activation of glial cells may be a two-edged sword. The activation of glial cells is important in limiting the damage and in protecting neurons and photoreceptors from the initial consequences of injury. In addition to maintaining homeostasis and restoring the blood-retina barrier, glial cell activation can mediate neural cell survival. Muller cell activation, induced by argon laser photocoagulation, slows photoreceptor cell death in the dystrophic retina of RCS rats [Chu et al., 1998]. Similarly, preconditioning with bright lights protects against light damage [Liu et al., 1998]. Injury-induced benefits are due to the release of neurotrophic factors from normal storage in glial cells [Mervin, 1999; LaVail, 1992]. In the therapy of retinal degenerations, injury-induced treatments have been proposed. Limited, shortlived, detachment of neural retina is part of a surgical procedure (macular translocation) for treating age-related macular degeneration [Fujii et al., 2002]. In traumatic injuries, however, the excessive and chronic activation of retinal glial cells contributes to negative remodeling of the retina with scar formation, neural cell death, and rewiring of the retina. These changes negatively alter the architecture and function of the retina. The clinical relevance of retinal healing and remodeling is substantial. The formation of fibrocellular membranes can lead to complications such as detachments, cell death, and ultimately visual impairment. The remodeling that occurs during wound healing can have unintended consequences affecting clinical strategies including surgical procedures, prosthetic implants, stem cell therapies, and gene rescue therapies [BeharCohen et al, 2000; Radner et al., 2001; Kinouchi et al., 2003].

While our knowledge of the morphological and cellular changes of reactive glial cells is extensive, a select few of the phenotypic changes has been related to molecular makers and regulatory mechanisms. Even when molecular markers have been identified we do not know (1) the context in which the molecular change occurs, (2) the relationship to global changes across 
the retina, and (3) the mechanisms that control these changes. Cataloguing the molecular changes that underlie the retinal healing response. Applying high level bioinformatics to these data can further reveal the mechanisms that control glial cell activation and cellular remodeling. This knowledge will be important in modulating glial cells during injury and therapeutic intervention, and perhaps in modulating glial cells in the survival of genes during retinal degenerations.

\section{Gene Expression Profiling}

One of the key factors in defining a cellular phenotype is the selective expression of specific genes as RNA transcripts. This collection of transcribed genes is known as the cell's transcriptome. Changes in gene expression levels contribute to specific phenotypic changes. Increased levels of Gfap mRNA in the retina, for example, precede and correlate with increased levels of GFAP immunoreactivity in Muller cells [Cao et al., 1997]. Defining the transcriptomewide changes that occur after retinal injury can reveal the molecular pathways that modulate retinal healing.

Transcriptome-wide analysis is feasible with DNA microarray technology. Microarray technology is a recent addition to the techniques exploiting a potent feature of complementary nucleic strands to form a duplex. Microarrays, like Southerns and Northerns, use complementary

oligonucleotides to probe the levels of specific RNA transcripts. In 1995, Schena and colleagues overcame the technical limitation of probing multiple gene targets by spotting complementary DNAs (cDNAs) into glass slides. A year later, Lockhart and colleagues monitored transcript expression using small, high-density arrays containing tens of thousands of synthetic oligonucleotides. They synthesized short nucleic probes on slides using a combination of photolithography and oligonucleotide chemistry. The advent of cDNA and oligonucleotide microarray opens the opportunity to profile transcriptome-wide changes that occur in the injured retina. 
The basic microarray technique involves RNA extraction, amplification, hybridization, and analysis. After RNA extraction, the RNA is copied, while incorporating either fluorescent nucleotides or a tag that is later stained with fluorescence [Butte, 2002]. The labeled, complementary RNA (cRNA) is then hybridized to a microarray for a period of time, after which the excess is washed off and the microarray is scanned under laser light. The fluorescence emitted from thousands of genes is recorded by a computer. Relative intensity units are placed into spreadsheets for comparisons among biological replicates and among experimental conditions. The analysis measures significant expression changes and extracts patterns of expression [Butte, 2002]. Significant expression changes are determined using fold changes (experimental condition versus control experiment) and statistical analyses (for example, Student's Test and Analysis of variance). The true power of microarray analysis comes from the analysis of many hybridizations to identify common patterns of gene expression. Statistical methods that identify similar patterns of expression are generally referred as "cluster analysis" [Quackenbush, 2001]. To interpret the results from the analysis of hundreds of genes across multiple experiments, it is helpful to generate intuitive visual representations. A commonly used approach relies on the creation of "dendograms" or "heat maps" in which each column of the matrix represents an experimental condition, and each row represents the expression pattern for a particular gene. In this dissertation, we use microarray technology to better understand the biology of retinal healing by defining the temporal changes in gene expression after retinal injury and by relating these changes to the inflammatory, proliferative, and remodeling phases of retinal healing.

\section{Genetics of Gene Expression}

Defining groups of genes with similar patterns of expression is not an endpoint in the analysis but a beginning. Gene expression profiles can be supplemented by higher-level analyses aiming at 
discovering the networks controlling transcript expression. One approach combines gene expression profiling with genetic linkage analysis to reveal the location of genetic elements that modulate expression levels in a genetically variable population [Davarsi, 2003; Broman, 2005]. Genetic analysis of gene expression is called "expression genetics" or "genetical genomics" [Broman, 2005]. Expression genetics has been used to identify genes underlying complex traits, including gene expression, disease, and behavior [Morley et al., 2004; Cheung et al., 2003; Hubner et al., 2005; Chesler et al., 2005; Bystrykh et al., 2005]. These studies showed that gene expression is highly variable and heritable and that the expression variability correlates to one or more regions of the genome. Some of these loci modulate genes within the interval (cis-acting) but most loci modulate genes at different genomic locations (trans-acting). The integrated gene expression profiling and linkage analysis has identified genes that predispose hypertension (Cd36), asthma (C5), and atherosclerosis (ABCG5 and $A B C G 8)$ [Aitman et al., 1999; Karp et al., 2000; Berge et al., 2000]. These studies illustrate the combined power of gene expression profiling and linkage analysis to study gene expression regulation at a greater scale and depth than is possible using either technique alone.

Of interest to the vision community is the regulatory loci that control gene expression in the CNS. Chesler and colleagues [2005] defined genome-wide regulatory loci that modulate gene expression in mouse forebrains. For their expression genetics study, Chesler and colleagues relied on a genetically variable population derived from the C57BL/6 and DBA/2J mouse strains, the $\mathrm{BXD}$ recombinant inbred $(\mathrm{RI})$ mouse strains. This reference population is of interest because the parental and BXD RI strains have considerable phenotypic differences related to CNS development and pathology. Chesler and colleagues documented that loci modulating geneexpression phenotypes are also related to neurological phenotypes, and that distinct regulatory loci modulate functionally related genes. For example, a group of genes involved in synaptic regulation forms a network modulated by distinct regulatory loci. Chesler and colleagues made their regulatory locus data publicly available at GeneNetwork (www.genenetwork.org). They also integrated their expression genetics data with the mapping for more than 650 phenotypes 
previously obtained from the BXD RI mouse strains. The GeneNetwork data can be valuable in identifying unknown molecular interactions. Based on gene expression covariance and the sharing of loci, the GeneNetwork data predicted an association between the nuclear protein $\mathrm{P} 2 \mathrm{P}-4$ and nuclear repressor PUM2. PUM2 pull-down methods combined with RT-PCR confirmed that PUM2 does indeed bind P2P-R mRNA [Scott et al., 2005]. In regards to retinal healing, the GeneNework data are of interest because the BXD RI mouse strains segregate phenotypic differences in audiogenic seizures, hearing loss, and neurogenesis in the adult hippocampus [Willott and Erway, 1998; Kempermann and Gage, 2002; Neumann and Collins, 1991]. Moreover, brains and spinal cords of C57BL/6 are resistant to neurotoxicity, whereas the brains and spinal cords of DBA/2J are more susceptible to neurotoxicity [Schauwecker and Steward, 1997; Inman et al., 2002]. Together these studies suggest that genetic variations between the C57BL/6 and DBA/2J alter the expression and function of master regulators of the CNS healing response. Mining the expression genetics data at GeneNetwork is expected to identify geneexpression phenotypes and regulatory loci that modulate the early events of the CNS healing response.

The ultimate goal of expression genetics is to identify the genes underlying polygenic traits and to gain a better understanding of their physiology and biochemistry. The BXD RI strains are an ideal genetic system to identify candidate genes. Each BXD RI strain was derived by inbreeding for over 20 generations an F1 cross between C57BL/6 and DBA/2J strains [Taylor et al., 1999]. The genome for each BXD RI strain is a unique recombination of the C57BL/6 and DBA/2J genomes. Since the parental genomes are sequenced and the segregation of BXD RI strains chromosomes is mapped, we have a complete map of all the candidate genetic variants responsible for the regulatory locus. A standard procedure to identify the best candidate gene is to define the polymorphic genes whose function is relevant to the phenotype [Abiola et al., 2003]. For example when the age-related maculopathy susceptibility locus was found, Hageman and colleagues [2005] focused on immune-associated genes to explain the aberrant complement activation seen in patients with macular degeneration. Higher resolution linkage mapping defined 
the complement factor $H$ gene as the polymorphic gene that predisposes individuals to agerelated macular degeneration. In our case, a candidate gene must be relevant to the biology of CNS healing, namely, is the gene known to be involved in CNS healing events and/or does its transcript change after trauma? The search for master regulators of CNS healing is facilitated by the growing collection of gene expression data that survey the CNS during development and after injury. These analyses can be further complemented with computational tools that examine the promoter sequence and the protein sequence of candidate modulators to determine if there are potential direct interactions between the candidate modulators and downstream targets [Segal et

al., 2005]. The convergence of gene expression profiling, expression genetics, and bioinformatics offers a novel and comprehensive approach to discover molecular pathways controlling retinal healing.

\section{Aims of the Dissertation}

The goal of this research is to define the molecular mechanisms that control the complex cellular events of retinal healing. The hypothesis is that the phenotypic changes that retinal cells undergo as part of the healing process are controlled by changes in their gene expression profile. This hypothesis is addressed by implementing an experimental design that integrates gene expression profiling, expression genetics, and bioinformatics. The experimental design to define master genes of retinal healing is outlined in the following aims:

Aim 1 (Chapter 2): Define the temporal profiles of gene expression after retinal injury using microarray technology, and relate these profiles to the known cellular changes of retinal healing.

Aim 2 (Chapter 3) Define regulatory loci that modulate wound-healing genes in mouse brains. We will mine expression genetics data by Chesler and colleagues (2005) to identify the genomic 
region that modulates genes expression in forebrains from mouse strains derived from the C57BL/6 and DBA/2J strains, the BXD recombinant inbred strains.

Aim 2 (Chapters 3 and 4). Define candidate upstream modulators within a regulatory loci by an integrated bioinformatic approach that combines databases that catalogue single-nucleotide polymorphisms, gene expression profiling of developing and injured CNS, and functional domains within promoters and proteins.

Aim 4 (Chapter 4): Test the effect of candidate gene dosage on wound healing using animal and cell culture systems. 


\section{Chapter 2}

\section{Temporal Changes in Gene Expression After Injury in the Rat Retina}

Permission to reproduce by Association for Research in Vision and Ophthalmology

Vázquez-Chona F, Song BK, Geisert EE. Temporal changes in gene expression after injury in the rat retina. Invest Ophthalmol Vis Sci. 2004; 45:2737-46. 


\section{Abstract}

Purpose: The goal of this study was to define the temporal changes in gene expression after retinal injury and to relate these changes to the inflammatory and reactive response. A specific emphasis was placed on the tetraspanin family of proteins and their relationship with markers of reactive gliosis.

Methods: Retinal tears were induced in adult rats by scraping the retina with a needle. After different survival times (4 hours, and 1, 3, 7, and 30 days), the retinas were removed; mRNA was isolated, prepared, and hybridized to Affymetrix RG-U34A chips. Microarray results were confirmed by using RT-PCR and correlated to protein levels.

Results: Of the 8,750 genes analyzed, approximately 393 (4.5\%) genes were differentially expressed. Clustering analysis revealed three major profiles. 1) The early response was characterized by the upregulation of transcription factors. 2) The delayed response included a high percent of genes related to cell cycle and cell death. 3) The late, sustained profile clustered a significant number of genes involved in retinal gliosis. The late, sustained cluster also contained the upregulated crystallin genes. When we examined the tetraspanins $C d 9, C d 81$, and $C d 82$, they were also associated with the late, sustained response.

Conclusion: The use of microarray technology allows us to define complex genetic changes underlying distinct phases of the cellular response to retinal injury. The early response clusters genes associated with the transcriptional regulation of the wound-healing process and cell death. Most of the genes in the late, sustained response appear to be associated with reactive gliosis. 


\section{Introduction}

In response to local injury, the retina responds with a characteristic series of changes at the site of injury. A secondary series of changes then spreads to involve the entire retina, often resulting in progressive degenerative changes and the formation of scar tissue [Ryan et al., 1994; Postel and Mieler, 1999; Gregor and Ryan, 1982; Fisher et al., 2001; Humphrey et al., 1993; Behar-Cohen et al., 2000]. These responses of the retina to injury can be divided into an early acute phase, a delayed subacute phase, and a late chronic phase [Ryan et al., 1994; Fisher et al., 2001; BeharCohen et al., 2000].

The early phase, which occurs within the first few hours after injury, is characterized by hemorrhage [Ryan et al., 1994; Gregor and Ryan, 1982; Humphrey et al., 1993], alterations in the glutamatergic system [Sherry and Townes, 2000], changes in ionic balance [Szabo et al., 1991], and the beginning of cell-death cascades [Grimm et al., 2000; Yoshimura et al., 2003]. It is during this early phase that the first changes in the transcriptome occur with upregulation of the immediate early genes [Grimm et al., 2000; Yoshimura et al., 2003]. The retina then undergoes a series of delayed cellular responses that last for days. Among these responses is a generalized inflammatory reaction in which damaged cells release pro-inflammatory cytokines that recruit peripheral blood components [Ryan et al., 1994; Gregor and Ryan, 1982; Behar-Cohen et al., 2000]. Many retinal cells experience cell-type-specific responses: dedifferentiation, degeneration, migration, hypertrophy, and proliferation [Fisher et al., 2001; Grimm et al., 2000; Bringmann and Reichenbach, 2001; Sahel et al., 1991]. For example, Müller glial cells and the retinal pigmented epithelium (RPE) enter a reactive state in which they change protein expression, proliferate, and migrate into the wound and vitreal space [Fisher, et al., 2001; Behar-Cohen et al., 2000; Bringmann and Reichenbach, 2001; Grierson et al., 1994]. Inflammation and cell proliferation resolve within the first week as the response to injury enters its chronic phase [Ryan et al., 1994; Fisher et al., 2001; Behar-Cohen et al., 2000]. During the late phase, RPE and Müller cells remain reactive and participate in structural remodeling of the retina. Cells that migrate into the wound and 
vitreal space replace the hemorrhage with fibrocellular membranes. With time, these membranes may contract, causing significant problems, including retinal detachment [Ryan et al., 1994; Fisher et al., 2001; Behar-Cohen et al., 2000; Grierson et al., 1994].

Progress in understanding the mechanisms controlling secondary injury has been significant. Gains have come about by focusing on individual molecules [Humphrey et al., 1993; Bignami and Dahl, 1979; Clarke and Geisert, 1998; Song et al., 2003] or groups of molecules [Sherry and Townes, 2000; Szabo et al., 1991; Grimm et al., 2000; Geller et al., 2003] and their participation in specific processes of the retinal healing response. However, many of the molecular events associated with activation of this response remain unknown. In the present study, we used microarray technology to catalogue the expression of thousands of genes after retinal injury. Our first approach was discovery-driven using the power of microarray to define the global patterns of gene expression changes. The second approach was hypothesis-driven focusing on the role of $C d 81$ (whose product is involved in proliferation and gliosis [Clarke and Geisert, 1998; Song et al., 2003; Dijkstra et al., 2000; Geisert et al., 1996; Sullivan and Geisert, 1998]) and markers of reactive gliosis, including the cytoskeletal protein glial fibrillary acidic protein (GFAP, [Ryan et al., 1994; Humphrey et al., 1993; Bringmann and Reichenbach, 2001; Bignami and Dahl, 1979; MacLaren, 1996; Miller and Oberdorfer, 1981]).

\section{Methods}

\section{Animals and Surgery}

We used a total of 59 male Sprague-Dawley (albino) rats (270-330 gm) and 2 Long Evans (pigmented) rats (350-400 gm), the latter to examine the RPE response to retinal injury (Table 2.1). We anesthetized 47 albino rats and 2 pigmented rats by intraperitoneal injection of a mixture of xylazine $(13 \mathrm{mg} / \mathrm{kg})$ and ketamine $(87 \mathrm{mg} / \mathrm{kg})$, then induced a retinal tear in both eyes of each rat. For this purpose, we used a 27-gauge needle to penetrate the pars plana, then scraped the superior 
Table 2.1. Experiments and distribution of animals.

\begin{tabular}{lll}
\hline Experiment & Rats & Survival time \\
\hline Gene expression & Albino: 30 injured, 6 control & 4 hours, and 1, 3, 7, and 30 days \\
Protein expression & Albino: 6 injured, 6 control & 7 days \\
Histology & Albino: 8 injured, 3 control & $1,3,7$, and 30 days \\
RPE response & Pigmented: 2 injured & 7 days \\
\hline
\end{tabular}

For the gene expression study, we used six animals per experimental condition to obtain three independent retinal samples with four retinas per sample. For each experimental condition in the protein expression study, we processed 3 independent retinal samples with two animals per sample. For histology of injured retina, we used two animals per experimental condition. 
temporal retina medially to laterally, as previously described [Clarke and Geisert, 1998; Song et al., 2003; MacLaren, 1996; Miller and Oberdorfer, 1981]. Care was taken to prevent lens and sclera damage. Twelve control animals received no injury. All animals were sacrificed by intraperitoneal injection of a mixture of xylazine $(26 \mathrm{mg} / \mathrm{kg})$ and ketamine $(174 \mathrm{mg} / \mathrm{kg})$. All protocols used in this study were approved by the Animal Care and Use Committee of the University of Tennessee Health Science Center and were in accordance with the ARVO Statement for the Use of Animals in Ophthalmic and Vision Research.

\section{Microarray Expression Profiles}

Arrays, Sample Preparation, and Hybridization. Using the rat RG-U34A oligonucleotide array (Affymetrix, Santa Clara, CA), we examined gene expression levels in the normal rat retina, and injured rat retinas with 4 hours and 1-, 3-, 7-, and 30-day survival time (Table 2.1). Retinas used for expression studies were dissected and examined under a stereomicroscope, SXZ12 (Olympus, Japan), and immediately processed. Nissl-stained sections of the eyecups showed that our retinal dissections included both the neural retina and RPE. We collected retinas from 36 animals -6 animals per experimental condition. For each experimental condition, we processed 3 independent retinal samples with 2 animals per sample. Thus, we collected 18 biological replicates and performed 18 hybridizations (Appendix B, Table B.1). For total RNA extraction, we used the TRIzol method (Life Technologies, Carlsbad, CA) and confirmed the integrity of RNA with the Agilent 2100 bioanalyzer (Agilent Technologies, Palo Alto, CA). We synthesized, labeled, and hybridized cRNA onto arrays at Genome Explorations (Memphis, TN) according to standard Affymetrix methods previously described by Rogojina et al. [2003].

Data Extraction and Normalization. We measured gene expression levels with Microarray Suite 5.0 (MAS 5.0; Affymetrix) and Robust Multi-array Average (RMA, [Irizarry et al., 2003]) algorithms. Signals were log transformed (base 2) and the mean intensity for each microarray was normalized to 8 ( $\log _{2}$ scale, [Rogojina et al., 2003]). 
Statistic and Clustering Analyses. We determined statistical significance of expression changes using Student's $t$-test statistics. To determine the expected proportion of significant false-positives, we used the false discovery rate of Benjamini and Hochberg [Reiner et al., 2003]. Significantly expressed genes were clustered with CLUSFAVOR 6.0 [Peterson, 2002] using principal component analysis (PCA). Averages are expressed as standard error means (SEM).

Minimal Information to Annotate a Microarray Experiment (MIAME) Compliance. For further discussion on experimental design and other details of methods, see Appendix A, MIAME report. The raw MAS 5.0 data set is publicly available at Gene Expression Omnibus (GEO, www.ncbi.nlm.nih.gov/geo) as the group series GSE1001 (for individual accession numbers see Appendix B, Table B.1).

\section{One-Step Reverse Transcription-Polymerase Chain Reaction}

Using fluorescent, one-step reverse transcription-polymerase chain reaction (RT-PCR), we verified microarray expression changes for early-response genes Fos and Fosll, delayed-response genes $I l l b$ and IrfI, and delayed-persistent response genes Cryab, Crygd, Cd81, and Gfap (nu-

cleotide sequences shown in Appendix B, Table B.2). We performed primer design, one-step RTPCR reaction, and RT-PCR analysis as previously described by Rogojina et al. [2003]. To eliminate genomic contamination, we treated total RNA with RQ1 RNase-Free DNase (Promega, Madison, WI). We performed RT-PCR reactions in the iCycler (BioRad, Richmond, CA) using the reagents in the SYBR Green RT-PCR kit (Applied BioSystems, Warrington, UK). To determine the relative change in gene expression, we compared the number of cycles $(\mathrm{Ct})$ needed to reach the midpoint of the linear phase using the iCycler analysis software. All observations were normalized to the housekeeping gene Rps 18 .

\section{Protein Expression and Localization}

To determine the levels of protein expression in normal retinas and those at 7 days after injury, we used rabbit polyclonal antibody against GFAP (Thermo Shandon, Pittsburgh, PA) mouse 
monoclonal antibody AMP1 that recognizes CD81 [Geisert et al., 1996], and rabbit polyclonal antibodies against crystallins $\square, \square$, and $\square$ (provided by Dr. Sam Zigler, National Institute of Health). The secondary antibodies were peroxidase-conjugated goat anti-rabbit IgG, goat antimouse IgG, and goat anti-mouse IgG (Jackson ImmunoResearch Laboratories, Inc., East Grove, PA), all of which had minimal cross-reactivity with rat serum proteins. We compared protein expression in injured retinas to control retinas using a previously described immunoblot method [Clarke and Geisert, 1998; Song et al., 2003; Geisert et al., 1996].

Indirect immunohistochemical methods were used to define the cellular localization of GFAP and crystallins in sections of normal and injured retinas. Injured rats had survival times of 1 day, 3 days, 7 days, and 30 days after retinal injury (Table 2.1). We anesthetized 8 injured rats and 3 control rats, processed tissue, and followed immunohistochemical methods as previously described [Clarke and Geisert, 1998; Song et al., 2003; Geisert et al., 1996]. For fluorescent microscopy, we used fluorescein-conjugated donkey anti-rabbit IgG (Jackson ImmunoResearch Laboratories, Inc.). To identify RPE cells, we used a rhodamine-conjugated lectin from Phaseolus vulgaris (PHA-E; Sigma-Aldrich, St. Louis, MO), and mouse monoclonal anticytokeratin antibodies (CBL 234 and MAB 3412; Chemicon, Temecula, CA). We labeled nuclei with a fluorescent Nissl counterstain, Hoechst 33342 (Sigma-Aldrich), and examined the sections with the Bio-Rad MRC-1024 confocal laser scanning microscope.

\section{Results}

\section{Glial and RPE Response to Retinal Injury}

To characterize the local and global response, we examined the distribution of retinal glia and RPE cell markers. In normal retinas, anti-GFAP labeled astrocytes and Müller cell endfeet at the ganglion cell layer (GCL; Figure 2.1A). PHA-E lectin stained the normal RPE, choroid, and outer segments (OS, Figure 2.1B) - a pattern similarly described by Cho et al. [2003]. 

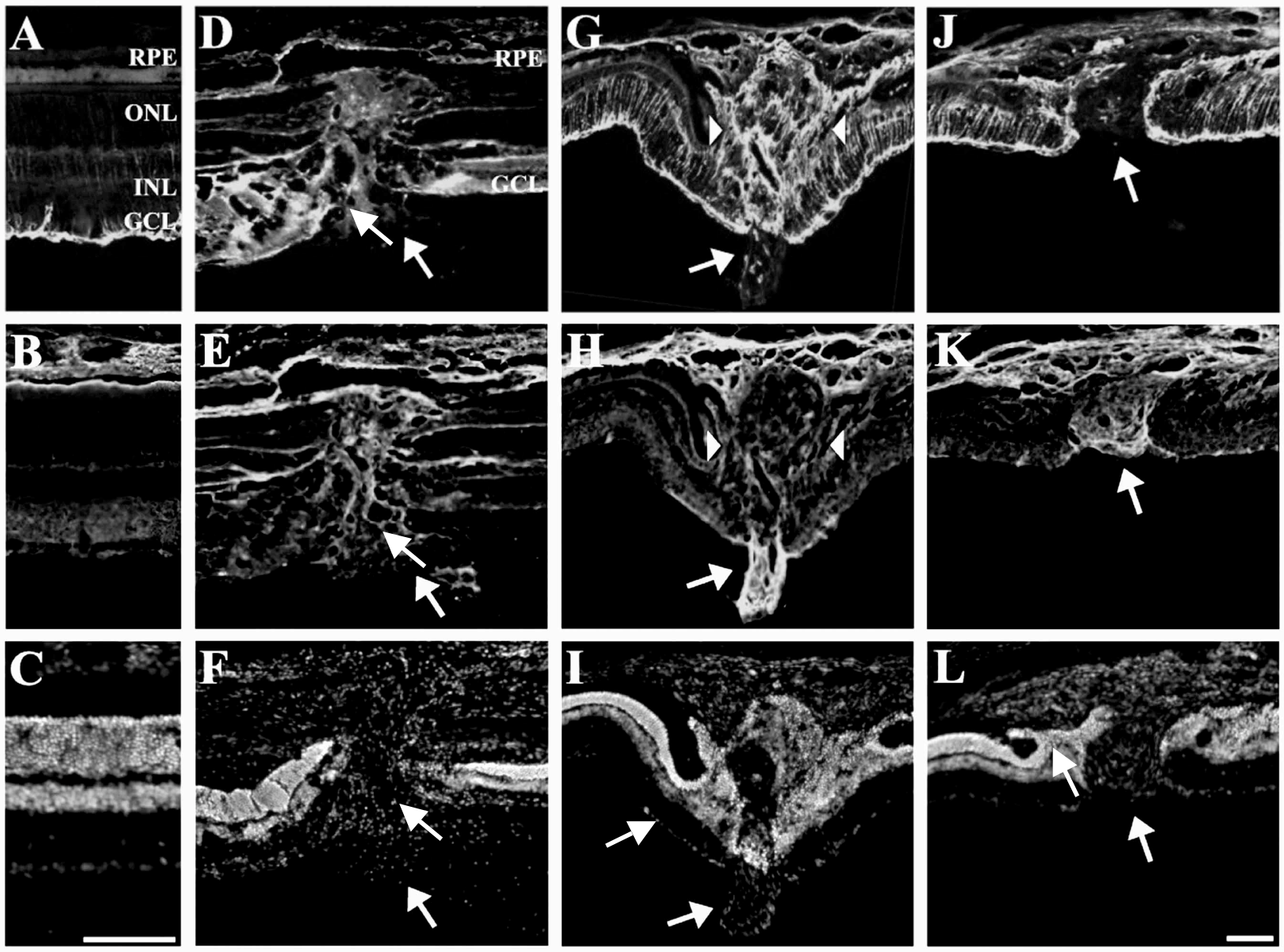

Figure 2.1. The cellular response of the rat retina to injury. Retinas labeled with anti-glial fibrillary acidic protein (GFAP; A, D, G, and $\mathbf{J}$ ), lectin from Phaseolus vulgaris (PHA-E; B, E, H, and $\mathbf{K})$, and Hoechst nuclear staining $(\mathbf{C}, \mathbf{F}, \mathbf{I}$, and $\mathbf{L})$. The figure illustrates 4 regions of retina: normal retina (A - C), retina 3 days after injury $(\mathbf{D}-\mathbf{F})$, and two different retinas 30 days after injury ( $\mathbf{G}$ - I and $\mathbf{J}$ - L). In normal retinas, anti-GFAP labeled astrocytes and Müller cell endfeet at the ganglion cell layer (GCL; A). PHA-E lectin stained the normal RPE, choroid, and outer segments (OS, B). At 3 days after injury, cells invading the retinal tear (F, arrow) labeled with anti-GFAP (D) arrow) or PHA-E lectin (E, arrow); whereas, near the injury anti-GFAP labeled mainly Müller cells and astrocytes at the GCL (D). At 30 days after injury, two cellular responses were observed at the site of injury. In one response, the cells filling the tear (I, arrows) were mainly glia cells ( $\mathbf{H}$, arrow heads). In the second response, RPE cells mainly filled the tear (K and $\mathbf{L}$, arrows). In some instances, epiretinal membranes with glia $(\mathbf{G}$, arrow) and RPE cells $(\mathbf{H}$, arrow) formed over the retinal surface. Panels $\mathbf{A}$ - $\mathbf{C}$ are of the same magnification with scale bar in $\mathbf{C}$ representing $50 \mu \mathrm{m}$. Panels $\mathbf{D}$ - $\mathbf{L}$ are of the same magnification with the scale bar in $\mathbf{L}$ representing $50 \mu \mathrm{m}$. 
After injuiry, the local response involved glia and RPE cells. As early as 3 days after injury, cells invading the retinal tear were labeled with anti-GFAP (Figure 2.1D, arrow) or PHA-E lectin (Figure 2.1E, arrow). Levels of GFAP and PHA-E labeling continued to increase so that by 30 days after injury, two cellular responses were observed at the site of injury. In one response, the cells filling the tear (Figure 2.1I, arrow heads) were mainly GFAP-positive glia cells (Figure 2.1H, arrow heads). In the second response, RPE cells mainly filled the tear (Figure $2.1 \mathrm{~K}$ and $1 \mathrm{~L}$, arrows) and glia cells formed a well-defined membrane separating the scar from the neural retina (Figure 2.1J). RPE cell response following retinal injury was consistent with the distribution of pigmented cells in injured Long Evans rats (Data not shown). In some instances, epiretinal membranes with glia (Figure 2.1G, arrow) and RPE cells (Figure 2.1H, arrow) formed projecting into the vitreous. Thus, local response to retinal injury involved glia and RPE cells in a manner consistent with previous studies [Song et al., 2003; Loiu et al., 2002].

In addition to the local response, we observed the spread of GFAP immunoreactivity throughout the retina. During the first 3 days after injury, GFAP labeling was limited to Müller cells adjacent to the injury and to astrocytes at the ganglion cell layer (Figure 2.1D). At 7 and 30 days after injury, we observed a global GFAP labeling of the retina localized to astrocytes at the ganglion cell layer, and to Müller cell processes (Figure 2.1G and 2.1J). Near the site of injury, GFAP labeling showed that the Müller cell processes became thicker and extended into the subretinal space, whereas GFAP labeling of peripheral Müller cells extended from the ganglion cell layer to the photoreceptor layer.

\section{Microarray Expression Profiles}

In our analysis of microarray data, we used two different tactics: a discovery-driven approach and a hypothesis-driven approach. In the discovery-driven approach, we defined different patterns of gene expression across the data set, clustering genes that might be functionally related. The hypothesis-driven approach focused on the gene expression of $C d 81$ and its relationship with reactive gliosis markers, particularly Gfap. This approach allowed us to concentrate on one aspect of 
the injury response to define the relationships within the tetraspanin family and markers of the reactive glial response.

Quality Controls. The expression profiles ( $\mathrm{n}=18$ arrays) met a strict set of quality-control parameters (Table 2.2). Housekeeping genes displayed consistent values and 5' to 3' ratios of less than 3. The average percentage of "Affymetrix present" calls across all arrays was $40.0 \pm 1.1 \%$. The intensity profiles ( $\log _{2}$ scale) for all the arrays showed a normal distribution with a mean at $6.59 \pm 0.04$ and an average standard deviation $\left(\square^{2}\right)$ of $2.32 \pm 0.04$. Comparison of expression profiles among arrays from independent replicates of the same experimental group $(n=3$ arrays/group) showed a within-group average correlation of $0.92 \pm 0.01$. Our quality controls are consistent with published microarray data [Yoshimura et al., 2003; Matzilevich et al., 2002; Di Giovanni et al., 2003]. Together, these results indicate integrity of starting RNA, and efficiency of first-strand cDNA synthesis and hybridization.

Data Extraction and Normalization. For temporal analysis of the data and comparison between data extraction methods, the mean intensity for each microarray was normalized to 8 ( $\log _{2}$ scale). This transformation yielded MAS 5.0 signal intensities ranging from 1 to 18 relative units of fluorescence. To determine a "present" signal threshold in our system, we plotted the coefficient of variation versus the averaged signal value and determined the threshold signal yielding stable coefficients of variation. From this analysis, the "present" signal threshold was set at 8.64. Below this threshold, signals displayed coefficients of variation greater than $10 \%$, while their variance increased exponentially (Appendix B, Figure B.1). Using this criterion, 4,480 $(50.9 \%)$ of the genes met the "present" criterion in at least one condition and were considered for further analysis. We compared the "present" expression signals to RMA values and found an average correlation of $0.92 \pm 0.01$ (Appendix B, Table B.3).

To further analyze the reliability of our "present" signals, we compared fold changes obtained with normalized MAS 5.0 and RMA to fold changes obtained with RT-PCR (Table 2.3). Using RT-PCR, we measured expression changes for Fos, Fosl1, Illb, Irf1, Cryab, Crygd, Cd81, and Gfap at 4 hours, 3 days, and 30 days after injury. For these genes, fold changes measured 
Table 2.2. Microarray expression profiles after an experimentally induced retinal tear.

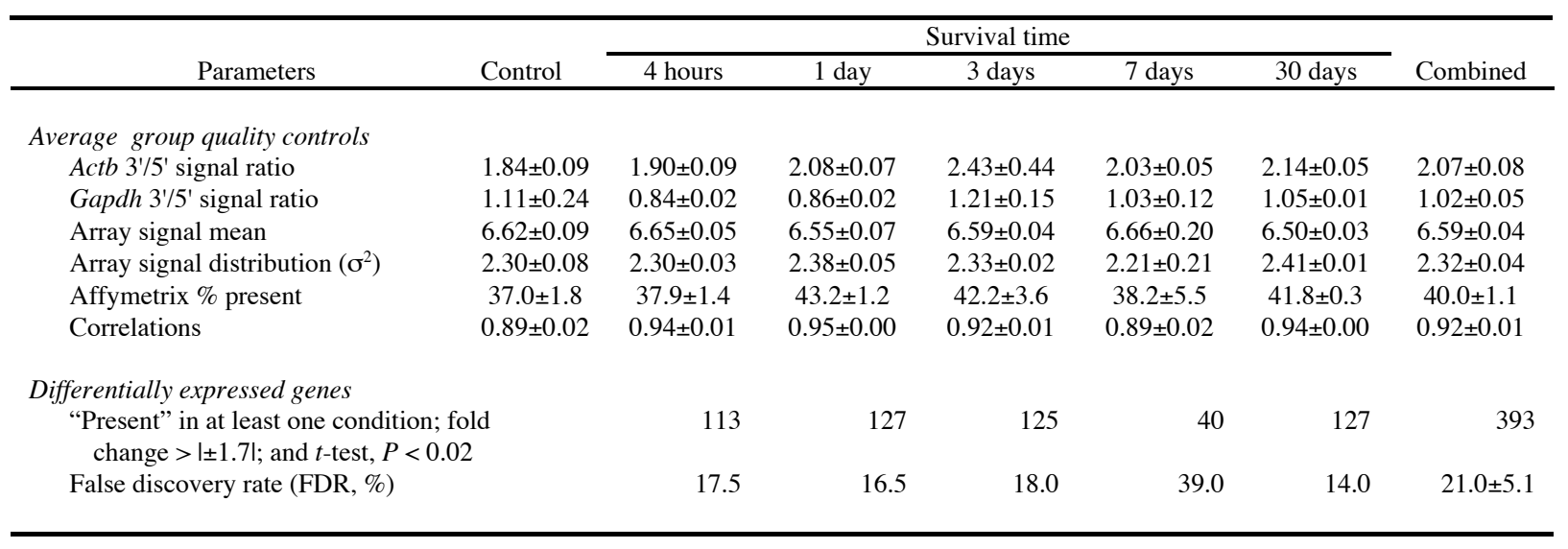

Three biological replicates were used per group. Averages are expressed as means \pm SEM. MAS 5.0 signals were transformed into the $\log _{2}$ scale. To determine temporal changes in gene expression, mean intensities were normalized to $8\left(\log _{2}\right.$ scale) and a signal "present" threshold was set at $8.64\left(\log _{2}\right.$ scale). The false discovery rate (FDR) of Benjamini and Hochberg [Reiner et al., 2003] was used to determine the expected proportion of false positives. While array expressions at 7 days post-injury displayed lower number of genes with significant expression changes and higher FDR, the three 7-day arrays met the quality controls, and the expression of house keeping genes (e.g., Gapdh, Actb, Ubc, Hprt, Sdha, and Rpl41) was consistent with expression found in the other experimental conditions. Thus, there was no empirical data (technical nor biological) to exclude any data from the 7-day experiments. 
Table 2.3. Transcript changes confirmed with real-time RT-PCR.

\begin{tabular}{lcccc}
\hline \multirow{2}{*}{ Gene } & Time & \multicolumn{3}{c}{$\log _{2}$ Fold Change } \\
& point & RT-PCR & MAS 5.0 & RMA \\
\hline Fos & $4 \mathrm{~h}$ & $1.9^{\dagger}$ & $2.1^{\dagger}$ & $1.2^{\dagger}$ \\
& $3 \mathrm{~d}$ & 0.3 & 0.1 & $(0.2)$ \\
& $30 \mathrm{~d}$ & 0.1 & 0.2 & 0.0 \\
Fosl1 & $4 \mathrm{~h}$ & $6.5^{\dagger}$ & $5.4^{\dagger}$ & $2.3^{\dagger}$ \\
& $3 \mathrm{~d}$ & $2.7^{\dagger}$ & 0.5 & 0.5 \\
& $30 \mathrm{~d}$ & $1.3^{\dagger}$ & $(0.3)$ & $(0 .)^{\dagger}$ \\
Il1b & $4 \mathrm{~h}$ & $2.1^{\dagger}$ & $2.0^{\dagger}$ & $1.4^{*}$ \\
& $3 \mathrm{~d}$ & $2.0^{*}$ & $1.2^{*}$ & $1.2^{*}$ \\
& $30 \mathrm{~d}$ & 0.5 & 0.1 & 0.7 \\
Irflb & $4 \mathrm{~h}$ & $1.0^{*}$ & $1.9^{*}$ & 1.0 \\
& $3 \mathrm{~d}$ & $1.2^{\dagger}$ & $2.3^{*}$ & $1.5^{*}$ \\
& $30 \mathrm{~d}$ & $0.1^{*}$ & $1.1^{*}$ & 0.2 \\
Cd81 & $4 \mathrm{~h}$ & 0.2 & 0.1 & $(0.1)$ \\
& $3 \mathrm{~d}$ & $1.0^{*}$ & $1.0^{*}$ & $1.2^{*}$ \\
& $30 \mathrm{~d}$ & $1.3^{\dagger}$ & $0.9^{*}$ & $0.7^{*}$ \\
Cryab & $4 \mathrm{~h}$ & $0.5^{*}$ & 0.8 & $0.9^{*}$ \\
& $3 \mathrm{~d}$ & $2.0^{\dagger}$ & $2.7^{*}$ & $2.6^{*}$ \\
& $30 \mathrm{~d}$ & $0.5^{*}$ & $2.4^{*}$ & $2.0^{*}$ \\
Crygd & $4 \mathrm{~h}$ & $(0.4)^{*}$ & $(0.7)$ & $(0.5)$ \\
& $3 \mathrm{~d}$ & $2.7^{\dagger}$ & $2.7^{\dagger}$ & $1.8^{\dagger}$ \\
& $30 \mathrm{~d}$ & $1.9^{*}$ & $3.1^{*}$ & $1.5^{*}$ \\
Gfap & $4 \mathrm{~h}$ & $1.6^{\dagger}$ & 0.4 & $0.4^{*}$ \\
& $3 \mathrm{~d}$ & $2.1^{\dagger}$ & $3.5^{\dagger}$ & $2.8^{*}$ \\
& $30 \mathrm{~d}$ & $2.3^{\dagger}$ & $4.1^{\dagger}$ & $3.8^{\dagger}$ \\
\hline \multirow{6}{*}{} & & & &
\end{tabular}

Fold changes measured with real-time RT-PCR were compared to those measured with the microarray U34A. Microarray signals were analyzed using Microarray Suite (MAS) 5.0 and robust multiarray average (RMA). Fold change represents $\log _{2}$ (injured/normal expression). Values in parentheses represent negative fold changes. Statistical significance compared population means between the control and each survival time (4h, 3d, and 30d): ${ }^{*} P<0.05$ and ${ }^{\dagger} P<0.01$. 
with normalized MAS 5.0 and RMA values were highly similar $(r=0.89)$. Both MAS 5.0 and RMA fold changes were similar in magnitude and direction to fold changes obtained with RTPCR. However, RT-PCR fold changes had higher correlations with normalized MAS 5.0 ( $r=$ 0.76) than with RMA $(r=0.58)$. Together, the high correlation between normalized MAS 5.0 values and RMA values and the similarity between microarray fold changes and RT-PCR fold changes confirm the reliability of our "present" signals, which eliminate genes with low expression signals. Thus, we have used the normalized MAS 5.0 values for our subsequent analysis.

\section{Discovery-Driven Analysis}

The overall goal of our discovery-driven approach was to define groups of genes with similar expression patterns. The first step in the analysis defined 393 genes with significant changes in expression levels (fold change $>1.7$ and $P<0.02$; Table 2.2). These differentially expressed genes represented an average false discovery rate of $21.0 \pm 5.1 \%$. Collectively, these genes displayed a range of dynamic expression changes across survival time. The second step involved clustering the 393 genes into groups according to the similarity of their expression profiles. Using principal component analysis, we identified 194 upregulated genes having transcription changes that conformed to one of three major profiles: an early response (Figure 2.2A), a delayed response (Figure 2.2B), and a late, sustained response (Figure 2.2C). Early response genes displayed a transient surge in expression following the retinal tear, whereas delayed response genes showed moderate upregulation at 4 hours and a delayed transient peak at either 1 or 3 days after injury. In contrast, late, sustained genes displayed peak upregulation at either 3 or 7 days and sustained over-expression at 30 days after injury. A complete list of upregulated genes organized by clusters is found in Appendix B, Table B.4.

The distribution of genes by functional category revealed that genes within clusters were functionally related (Figure 2.2D). For instance, $75 \%$ of the upregulated transcription genes clustered in the early response profile. Additional functional groups represented in the early response included cell proliferation, apoptosis, and cell-survival mechanisms. Genes 

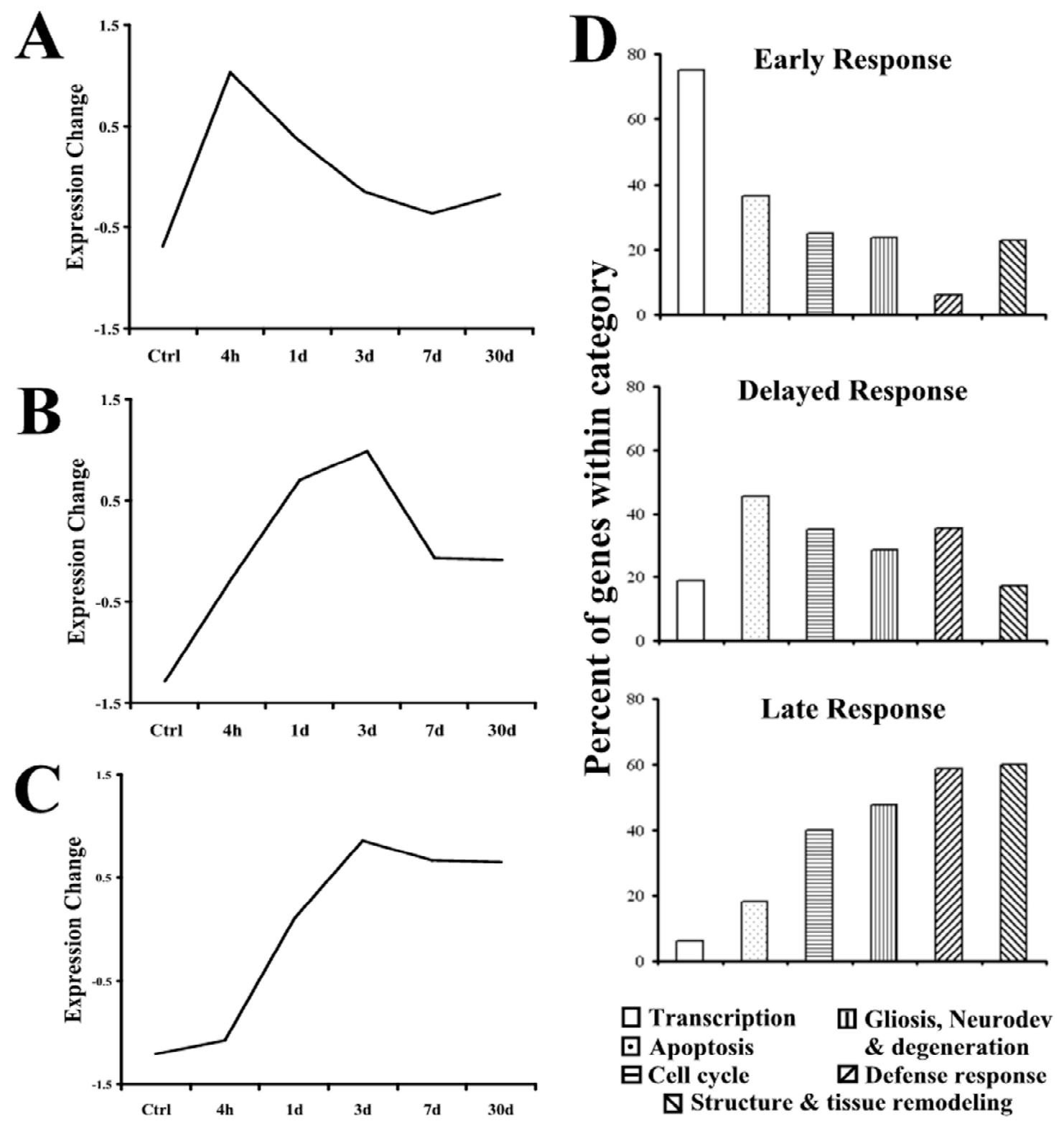

Figure 2.2. Gene expression profiles after retinal injury. Differentially expressed genes $(n=194)$ were clustered into 3 major profiles: an early response (A), a delayed response (B), and a late, sustained response $(\mathbf{C})$. Genes were clustered using principal component analysis. Early-response genes displayed a transient surge in expression after injury. Genes fitting the delayed-response profile had a transient peak in expression at either 1 or 3 days after injury. Genes within the third expression profile had a late expression peak (i.e., at either 3 or 7 days) and sustained overexpression at 30 days after retinal injury. A complete list of upregulated genes organized by clusters is found on Appendix B, Table B.3. The distribution of genes by functional category reveals that those within each expression profile are functionally related $(\mathbf{D})$. 
highly represented in the delayed response profile included those regulating cell cycle, cell death and survival, neural development, and gliosis. A high percentage of late, sustained genes were associated with the reactive responses of the retina: gliosis, inflammation, stress response, neuronal degeneration, and tissue remodeling. A summary and expanded version of the distribution of genes by clusters and functional category are shown in Figure 2.3 and Appendix B, Table B.5, respectively.

One unexpected finding was the clustering of the crystallin genes with the retinal reactive marker Gfap. All the crystallins in the microarray-Cryaa, Cryab, Cryba1, Cryab4, Crybb2, Crybb3, Crygc, Crygd, and Cryge-displayed strong, sustained upregulation (Appendix B, Table B.5, category 7). To confirm our findings, we examined the mRNA and protein expression of selected crystallins. We measured the mRNA expression of Cryab and Crygd, using RT-PCR. Cryab mRNA levels showed a moderate fold increase of $1.42 \pm 0.11$ at 4 hours after injury, a robust upregulation of $4.13 \pm 0.12$ at 3 days, and a moderate fold change of $1.46 \pm 0.04$ at 30 days (Figure 2.4A). Crygd mRNA showed an initial down-regulation of $1.34 \pm 0.03$, followed by significant changes to $6.36 \pm 0.25$ at 3 days and $3.64 \pm 0.41$ at 30 days (Figure 2.4A). Our microarray and RT-PCR measurements showed agreement in the direction of change of Cryab and Crygd (Figure 2.4A). Moreover, the mRNA expression of Cryab and Crygd across time displayed a pattern of expression that was similar to that of genes in our late, sustained profile, with a delayed peak at 3 days and strong, sustained upregulation even after 30 days.

To extend our crystallin gene findings, we measured the protein levels of the crystallins $\square$, $\square$, and $\square$ using a quantitative immunoblot method (Figure 2.4B). The immunoreactivity of the crystallins significantly increased to $7.1 \pm 0.39,6.8 \pm 0.66$, and $11.1 \pm 2.4$, respectively, at 7 days after injury. Taking this analysis one step further, we defined the localization of the reactivity of crystallins $\square, \square$, and $\square$ in normal and injured retinas. In normal retinas, we observed labeling of crystallins $\square, \square$, and $\square$ mainly at the ganglion cell layer (Figure 2.4C, 4E, and 4G). The crystallins had well-defined patterns of immunoreactivity at the site of injury and within the layers of the retina. At 7 and 30 days following injury, crystallin immunoreactivity was seen throughout 


\title{
Early Delayed Late \\ Transcription \\ Fos, Fosl1, Egrl, Nr4al, Cebpd, \\ Cebpd, Nfkb1,Stat3, Irf1, and Zfp36 \\ Isl1, Nfkb1,Stat3, Irfl, Zfp36, \\ Atf3, Cbfb, Crem, and Rnf4
}

\section{Cell cycle \& growth}

\author{
Akap12, Pak3, Btg2, Ssecks322, Arhgap7, Igf1, Igfals, and Insig1 \\ Akap11, D123, Dlg1, Itgb1, Lcn2, Ptn, Ywhae, \\ Ywhaq, Abi2, Nap1l1, and $V h l$ \\ Cd82, Ppap2b, and Prkr
}

\section{Apoptosis \& neurodegeneration}

Bax, Casp3, Ctsl, Litaf, Tnfrsf21, Mx3, Apobec1, Vdac1, A2m, and Spinc2

Stress response \& cell survival

Btg2, Eefla2, Parp, Snca, Apoe, Mt1a, Sod2, Dnajb9, Hspala,Cp, Clu, Cryab, and Gsta1

\section{Inflammatory response}

Cxcl10, Il2r, Ptgs2, RT-BM1, Il6r, Cish3, Cst4,

Ctsc, Illb, Lman1, and Pros1,

Fcgr3, Gtpi, Ifitm3l, RT1Aw2, Clqb, Clr, C1s, C3, Cd48, Cd74, Edn2, RT1.C/E, RT1.Da u, RT1.Mb, Scyb13, and Snn

\author{
Gliosis \& Neural development \\ Cstb, Jag1, Ania4, Cntn1, Vcam1, and Ninj1 \\ A2m, Anxa5, Apoe, Cd81, Cp, Clu, Cntf, Cryab, Dpysl4, \\ Gfap, mrf-1, Pea15, Pmp22, S100al, and Sparc \\ Structure \& tissue remodeling \\ Krt1-14, Plat, Tpm3, IMPG2, and Lim2 \\ Tubb1, Fuca, Lip1, Arpclb, Bfsp1, Ctsh, Ctss, Fmod, Grifin, \\ $L y z, M g p$, Myo5b, Nedd4a, and Tpm3
}

Figure 2.3. Temporal distribution of genes and functional categories after retinal injury. Location of genes describes relative gene expression peaks: early expression corresponds to peak expression at 4 hours; delayed expression corresponds to peak expression at 1 or 3 days; and late expression corresponds to peak expression at 7 or 30 days. For specific expression profiles see Appendix B, Table B.4. 

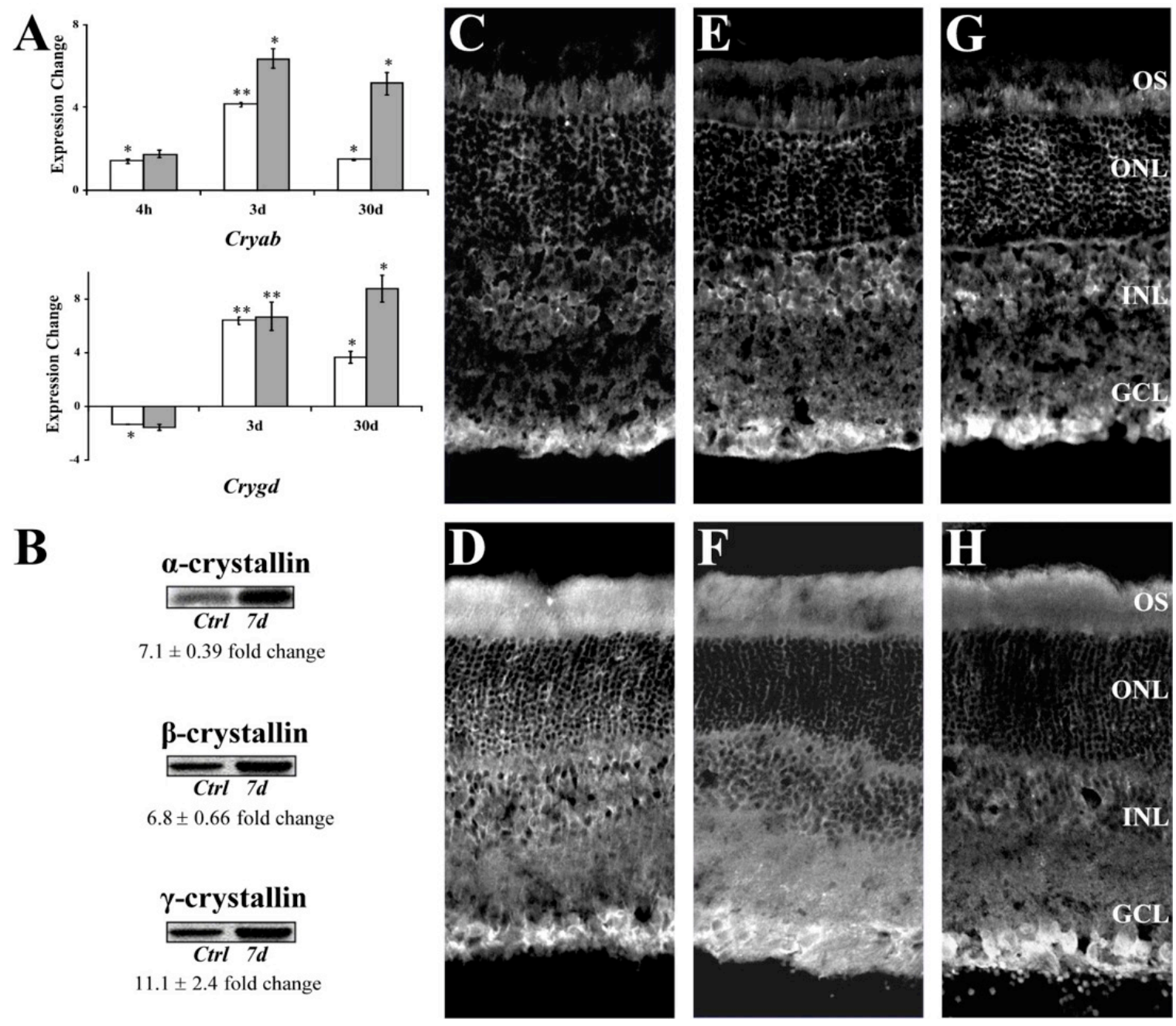

Figure 2.4. The crystallins $\square$, $\square$, and $\square$ were dramatically upregulated after retinal injury. The microarray expression changes for Cryab and Crygd (A, gray bars) were confirmed with RT-PCR (A, white bars). Immunoblots show increased levels of crystallin $\square$, $\square$, and $\square$ proteins (B). Crystallin $\square, \square$, and $\square$ expression in normal retinas $(\mathbf{C}, \mathbf{E}$, and $\mathbf{G}$, respectively) and injured retinas 7 days after injury (D, F, and $\mathbf{H}$, respectively) was localized by immunohistochemistry. In normal retina, crystallin immunoreactivity was present mainly at the ganglion cell layer (GCL; $\mathbf{C}, \mathbf{E}$, and G). Seven days after injury, there was a strong crystallin immunoreactivity throughout the scar spanning the retinal tear and protruded into the vitreal space (data not shown). High immunoreactivity levels for crystallins $\square(\mathbf{D}), \square(\mathbf{F})$, and $\square(\mathbf{H})$ were found immediately adjacent to the retina at GCL and outer segment layer (OS). No staining was seen when secondary antibody control was used (data not shown). Asterisks denote significant changes from the normal $(t$-test, $* P<$ $0.05, * * P<0.01)$. 
the scar that spanned the retinal tear and protruded into the vitreal space. Immediately adjacent to the injury, strong crystallin immunostaining was present at the ganglion cell layer and the photoreceptor layer (Figure 2.4D, 4F, and 4H; 7-day retinas shown). Moderate levels of immunoreactivity were also observed throughout the inner plexus layer, the inner nuclear layer, and the RPE. The well-defined pattern of crystallin mRNA upregulation by microarray and RT-PCR as well as the increase of crystallin protein and crystallin localization in the retina by immunohistochemical methods validate our discovery-driven observations that crystallins displayed strong, sustained transcription in response to injury in the rat retina.

\section{Hypothesis-Driven Approach}

The primary interest of our laboratory is the role of CD81 in the healing response of the retina. We specifically examined the relationships between $C d 81$, other tetraspanins, and markers of reactive glia. Our microarray data showed that $C d 81$ was moderately upregulated at 4 hours, to $1.05 \pm 0.1$, and significantly increased to $1.99 \pm 0.2$ and $1.88 \pm 0.1$ at 3 and 30 days, respectively (Figure 2.5A, gray boxes). RT-PCR confirmed the sustained upregulation of $C d 81$ mRNA after retinal injury (Figure 2.5A, white boxes). Our analysis showed that $C d 81$ clustered in the late, sustained profile, along with the tetraspanins and several glial reactive markers (see Appendix B, Table B.4, genes 126-194). We used a second round of PCA to define further the associations between $C d 81$ and the late, sustained profile genes. The subclusters were visualized by plotting the first two components (Figure 2.5B). Cd81 clustered tightly with tetraspanins $C d 9$ and $C d 82$, as well as with the genes for associated proteins MHC class I (RT1Aw2 and RT1.Dau) and MHC class II $(R T 1 . C / E)$. Together, these genes displayed persistent changes after retinal injury, with peak expression occurring at 3 days after injury. Tetraspanins $C d 37, C d 53$, and $C d 151$ were present in the normal retina, but their expression remained constant following injury. Among the glial reactive markers, Anxa5, Cntf, Pea15, Pmp22, S100a1, and Sparc clustered tightly with Cd81, whereas Clu, Cryab, Gfap and $A 2 m$ formed a separate cluster within the late sustained profile (Figure 2.5B). 

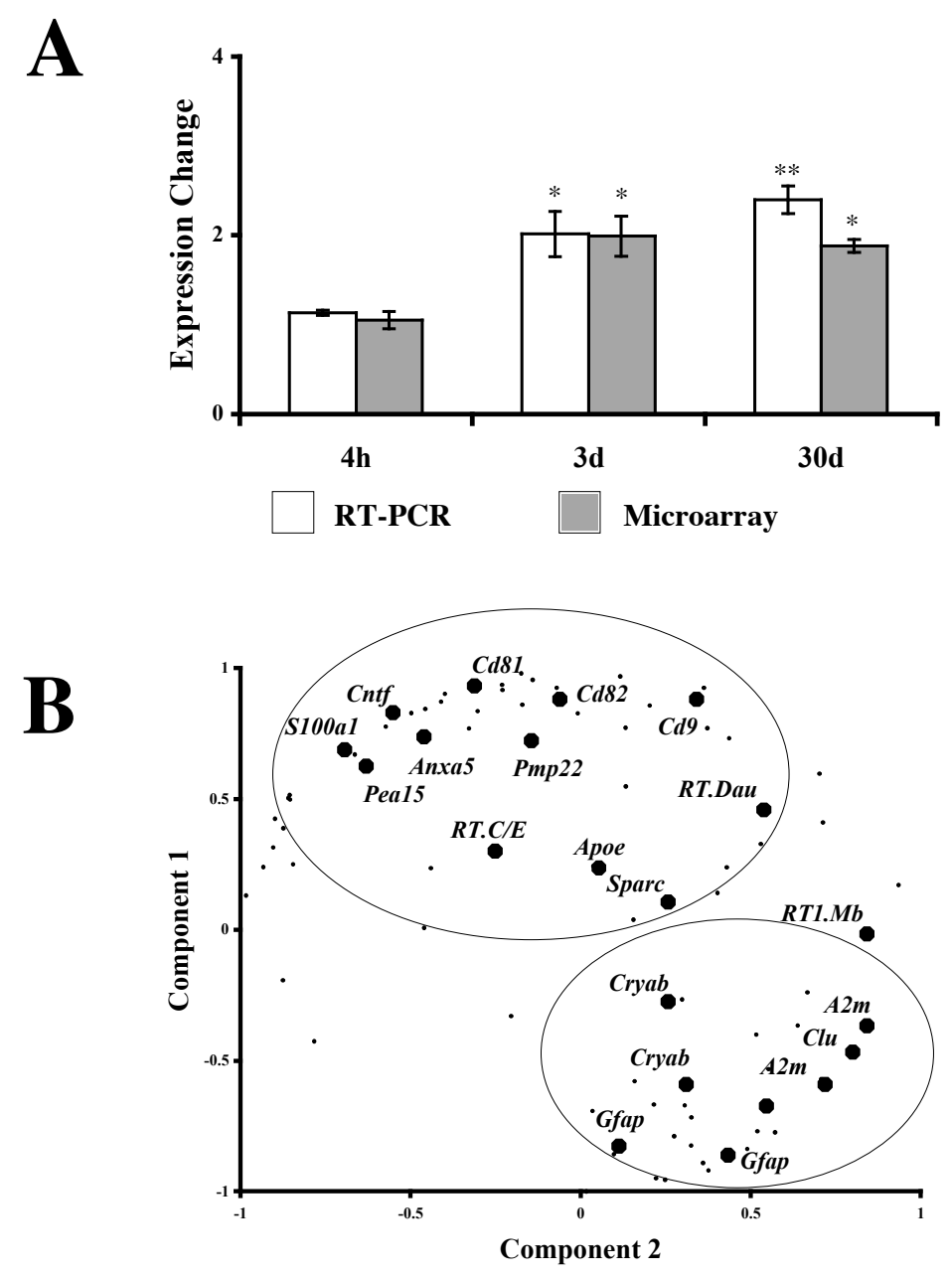

Figure 2.5. $C d 81$ clustered into the late-response profile along with tetraspanins and glial reactive markers. RT-PCR confirmed the sustained upregulation of $C d 81$ after injury (A). A second round of principal component analysis was used to define further the associations between $C d 81$ and the genes within the late-response profile. The subclusters were visualized by plotting the first two components (B). $C d 81$ clustered tightly with tetraspanins $C d 9$ and $C d 82$, as well as with the genes for associated proteins MHC class I (RT1Aw2 and RT1.Dau) and MHC class II $(R T 1 . C / E)$. Asterisks denote significant changes from the normal $(t$-test, $* P<0.05, * * P<0.01)$. 


\section{Discussion}

Our microarray analyses describe both a local reactive response at the site of retinal injury as well as changes occurring across the entire retina. As with injuries in other parts of the central nervous system (CNS), a local glial response is identified by the dramatic upregulation of the cytoskeletal protein GFAP [Ridet et al., 1997]. In the retina, this occurs in both Müller cells and astrocytes [Fisher et al., 2001; Humphrey et al., 1993; Bringmann and Reichenbach, 2001; 21]. Reactive Müller glia cells hypertrophy, proliferate, and form a protective barrier around the damaged area [Humphrey et al., 1993; Bringmann and Reichenbach, 2001; MacLaren, 1996]. Studies done in our laboratory [Song et al., 2003] and by others [Humphrey et al., 1993; MacLaren, 1996] have identified the spread of GFAP reactivity over the entire retina even after a modest local insult. This global GFAP response has been observed in association with various types of injury, including retinal detachment [Fisher et al., 2001], mechanical injury [Clarke and Geisert, 1998; Song et al., 2003; MacLaren, 1996; Miller and Oberdorfer, 1981] laser lesions [Humphrey et al., 1993], and optic nerve injury [Bignami and Dahl, 1979], as well as after transplantation of retinal cells [Behar-Cohen et al., 2000]. Widespread spatial changes have also been reported for CD81 [Song et al., 2003], heat shock protein 27 [Strunnikova et al., 2001], clusterin [Wong et al., 2001], and NF- $\square$ B [Geller et al., 2003; Wu et al., 2002]. Therefore, by examining changes over the entire retina, we measured in a consistent way the local and global response to the retinal tear.

The initial set of differentially expressed genes included 393 genes, $4.5 \%$ of the genes in the RG-U34A chip. Using clustering analysis [Peterson, 2002; Di Giovanni et al., 2003; Heyer et al., 1999], we discovered three major expression profiles: an early response, a delayed response, and a late, sustained response. Genes within each of the profiles were functionally related. The early response profile reflects rapid transcription of the immediate early genes as well as genes involved in cell proliferation, apoptosis, and cell survival. Genes highly represented in the delayed profile included those with functions relating to the cell cycle, cell death and survival, neural development, and gliosis. Within the late, sustained profile, a high percentage of genes were 
associated with the reactive response of the retina: gliosis, inflammation, stress response, neuronal degeneration, and tissue remodeling. The clustering of functionally related genes during retinal wound healing suggests that genes within a profile may be co-regulated. Others have identified transcriptional networks controlling clusters of genes expressed during the cell-cycle progression in yeast Saccharomyces cerevisiae [Heyer et al., 1999, Spellman et al., 1998]. Spellman and associates also found that genes in a given cluster share common regulatory motifs in the promoter region [Spellman et al., 1998]. Ongoing work in our laboratory reveals that selected genes within a cluster may be under the control of a common regulatory network [Geisert EE, et al. IOVS 2003 44: E-Abstract 2967].

The temporal regulation of genes and functional groups we observed is consistent with previous studies of gene expression in the retina [Grimm et al., 2000; Yoshimura et al., 2003], brain [Matzilevich et al., 2002], and spinal cord [Di Giovanni et al., 2003]. For example, early gene expression of the transcription factors c-Fos, Fosl1 (Fra-1), Nfkb1, Stat3, Egrl (Krox24/NGFI-A), Cebpd, Atf3, and Irfl has been reported in response to retinal ischemia [Yoshimura et al., 2003], photopic injury [Grimm et al., 2000], and retinal detachment [Fisher et al., 2001; Geller et al., 2003]. Moreover, the progression of gene expression changes in our study reflects the biochemical, cellular, and morphologic changes previously reported [Ryan et al., 1994; Fisher et al., 2001; Humphrey et al., 1993; Sherry and Townes, 2000; Szabo et al., 1991; Grimm et al., 2000; Yoshimura et al., 2003; Bringmann and Reichenbach, 2001; Grierson et al., 1994; Sahel et al., 1991; Geller et al., 2003]. An initial inflammatory response [Ryan et al., 1994; Humphrey et al., 1993; MacLaren, 1996] and change in ionic balance [Sherry and Townes, 2000; Szabo et al., 1991] are followed by a series of cellular responses that include RPE and Müller cell reactivity and proliferation, and cell death [Fisher et al., 2001; Grimm et al., 2000; Bringmann and Reichenbach, 2001; Grierson et al., 1994; Sahel et al., 1991]. During the late response, the reactive RPE and Müller cells participate in structural remodeling of the retina, including the formation of fibrocellular membranes [Ryan et al., 1994;Fisher et al., 2001; Behar-Cohen et al., 2000; Grierson et al., 1994]. Our analysis demonstrates that microarray clustering analysis can identify 
components of biochemical complexes and cellular pathways involved in the response of the retina to injury.

Given the importance of Müller cells, astrocytes, and microglia in the response of the retina to injury, genes associated with reactive gliosis are of particular interest. In this study, a significant group of gliosis-related genes clustered with Gfap in the late, sustained profile (Figure 2.3). Several of these gliosis-related factors, including Cp [Chen et al., 2003], CNTF [Wang et al., 2002], ApoE [Anderson et al., 2001], CLU [Wong et al., 2001] Anxa5 [Yoshimura et al., 2003], S100 [Sahel et al., 1991], and SPARC [Gilbert et al., 1999], are known to be expressed by reactive Müller and RPE cells. Whereas $m r f-1$, Pea15, and Pmp22 genes were unexpectedly upregulated in the retina, although they are expressed by reactive glia elsewhere in the nervous system [Tanaka et al., 1998; Trencia et al., 2003; Atanasoski et al., 2002]. Potential factors controlling glial activation in the retina include cytokines and inflammatory transcription factors. Cytokines and their receptors that are upregulated in our microarray data and are thought to play a key role in the modulation of glial cell function include IL-1 1 , IL-6R, and CNTF [Wang et al., 2002; Acarin et al., 2001]. The actions of these cytokines in glia cells are mainly mediated by the transcription factors STAT3 and NF- $\square$ B [Acarin et al., 2001]. For example, intravitreal injections of CNTF or IL-6 activate STAT3 in Müller cells [Wang et al., 2002]. Among the reactive glia genes targeted by STAT3 include Gfap, A2m, Fos, Irf1, and Stat3 [Wang et al., 2002; Acarin et al., 2001; Horvath, 2001]. In addition, Müller cells show NF- $\square$ B activation within hours after retinal detachment [Geller et al., 2003]. Noteworthy, NF- $\square$ B also modulates the expression of Gfap as well as $I l 1 b$ and Il6 [Acarin et al., 2001]. Together, these data suggest that STAT3 and NF- $\square$ B mediate signaling pathways involved in the glial response to retinal injury.

One novel revelation in our analysis was the profound upregulation of crystallins $\square, \square$, and $\square$ (Figure 2.2). Traditionally, crystallins have been thought to be lens-rich proteins involved in lens development and maintenance. However, recent studies suggest that crystallins have broader roles and are expressed beyond lens tissue [Yoshimura et al., 2003; Horwitz, 2003; Piatigorsky, 1998; Alge et al., 2002; Sakaguchi et al., 2003; Xi et al., 2003]. Gene-sequence homol- 
ogy studies of crystallins show that members of this family display considerable homology with genes involved in the stress response [Horwitz, 2003; Piatigorsky, 1998]. For instance, the $\square-$ crystallins belong to the small heat-shock protein family and may act as molecular chaperones [Horwitz, 2003; Piatigorsky, 1998]. In cultured RPE, —B-crystallin upregulation confers resistance to stress-inducible apoptosis [Alge et al., 2002]. A recent gene expression study confirmed the increased expression of selected $\square, \square$, and $\square$ crystallin transcripts in the rat retina after ischemia-reperfusion injury [Yoshimura et al., 2003]. Similarly, we found that the mRNA of all ten crystallins represented in the microarray displayed strong, sustained upregulation after retinal injury. These results are consistent with the increased crystallin protein expression confirmed by Sakaguchi and colleagues using mass spectrometry after exposing rat retina to light injury [Sakaguchi et al., 2003]. Furthermore, the temporal and spatial expression of crystallins is similar to that of GFAP, implicating this family of proteins in both the local and global response of the retina to trauma. Notably, studies of gene expression in the brain [Matzilevich et al., 2002] and spinal cord [Di Giovanni et al., 2003] using the identical microarray platform (RG-U34A) did not find a similar upregulation in crystallins $\square, \square$, and $\square$ in response to trauma. While crystallin expression in normal mouse retina displays animal-to-animal variation [Xi et al., 2003], studies after retinal detachment [Yoshimura et al., 2003], light injury [Sakaguchi et al., 2003], and our retinal tear consistently report the upregulation of crystallins. The coordinated upregulation of crystallins after injury is a potentially important part of the retinal wound-healing process that merits further investigation. Defining the response of the crystallin family to injury illustrates the power of microarray technologies and the discovery-driven approach.

Microarray technology, combined with gene expression profiling, offers the potential to identify cellular pathways and molecular complexes that are important in the regulation of retinal responses to trauma and disease [Grimm et al., 2000; Yoshimura et al., 2003]. Our laboratory is defining the role of CD81 in reactive gliosis in the retina [Clarke and Geisert, 1998; Song et al., 2003], brain [Sullivan and Geisert, 1998], and spinal cord [Dijkstra et al., 2000]. CD81 is part of a molecular complex within the plane of the membrane that links adhesive interactions into sec- 
ond-messenger cascades [Boucheix et al., 2001; Hemler, 2001]. Using microarray technology, we monitored gene expression changes for $C d 81$ and the entire family of tetraspanins (e.g., $C d 9$, Cd37, Cd53, Cd63, Cd81, Cd82, and Cd151) [Boucheix et al., 2001; Hemler, 2001]. In addition, we specifically mined the data to define changes in molecules known to associate with tetraspanins to determine if the members of the tetraspanin complex are co-regulated. Our analysis indicates that similar patterns of expression are shared by the tetraspanin genes $C d 9, C d 81$, and $C d 82$, as well as genes for the major histocompatibillity complex (MHC) receptors $R T 1 A w 2$, RT1.Dau, and RT1.C/E (Figure 2.3). Tetraspanins CD81, CD9, and CD82 are known to associate with MHC receptors in a number of different cell types, including lymphocytes, endothelial cells, and oocytes [Boucheix et al., 2001; Hemler, 2001; Szollosi et al., 1996]. For instance, CD81 and CD82 interact with MHC class I and II in antigen-presenting cells to modulate signaling through MHC molecules and antigen presentation [Szollosi et al., 1996]. Defining the temporal expression of tetraspanins and molecules to which they complex allows us to focus on the specific role of CD81 in the retina, its potential molecular associations, and its role in the reactive response.

The present study catalogued the time course of expression changes in response to a retinal injury. By surveying the expression patterns of 8,750 genes, we defined an early, a delayed, and a late, sustained response to retinal injury and demonstrated that within each profile, genes participate as functional units involved in different aspects of the healing response. Within the late response profile, we identified a host of glial reactive genes as well as crystallins, a previously overlooked family of proteins in the injured retina. In profiling the expression of tetraspanins, we identified CD9, CD81, and CD82 as potential organizers of molecular complexes regulating proliferation and the glial activation response. Our finding that crystallins and tetraspanins can act in functional units highlights the fact that the function, or therapeutic intervention, of a gene can be regulated at the gene expression level. However, we are limited in our ability to link crystallins or tetraspanins to specific cell populations or specific pathways. Future research using single-cell studies and genetically-altered animals will help dissect the molecular pathways and networks regulating gene expression. 


\title{
Chapter 3
}

Genetic Networks

\section{Controlling Retinal Injury}

\author{
Permission to reproduce by Molecular Vision
}

Félix R. Vázquez-Chona, Amna N. Khan, Chun K. Chan, Anthony N. Moore, Pramod K. Dash,

M. Rosario Hernandez, Lu Lu, Elissa J. Chesler, Kenneth F. Manly, Robert W. Williams, and Eldon E. Geisert, Jr. Mol Vis. 2005;11:958-970. 


\section{Abstract}

Purpose: The present study defines genomic loci underlying coordinate changes in gene expression following retinal injury.

Methods: A group of acute phase genes expressed in different nervous system tissues was defined by combining microarray results from injury studies from rat retina, brain and spinal cord. Genomic loci regulating the brain expression of acute phase genes were identified using a panel of $\mathrm{BXD}$ recombinant inbred (RI) mouse strains. Candidate upstream regulators within a locus were defined using single nucleotide polymorphism databases and promoter motif databases.

Results: The acute phase response of rat retina, brain, and spinal cord was dominated by transcription factors. Three genomic loci control transcript expression of acute phase genes in brains of BXD RI mouse strains. One locus was identified on chromosome 12 and was highly correlated with the expression of classic acute phase genes. Within the locus we identified the inhibitor of DNA binding $2(I d 2)$ as a candidate upstream regulator. $I d 2$ was upregulated as an acute phase transcript in injury models of rat retina, brain, and spinal cord.

Conclusion: We defined a group of transcriptional changes associated with the retinal acute injury response. Using genetic linkage analysis of natural transcript variation, we identified regulatory loci and candidate regulators that control transcript levels of acute phase genes. 


\section{Introduction}

To understand the global consequences of retinal injury, several laboratories monitored the transcriptome with microarray techniques [Vazquez et al., 2004; Yoshimura et al., 2003; Ahmed et al., 2004; Wilson et al., 2003; Chen et al., 2004]. Changes in transcripts were analyzed to define the key regulatory events following retinal injury. In an earlier study, we defined a temporal grouping of changes related to the biochemical and cellular response to retinal injury [Vazquez et al., 2004]. This type of analysis, defining gene clusters that occur after injury, is not an endpoint in the analysis but a beginning. The growing number of bioinformatic resources provides an opportunity to further analyze and interpret the changes in the transcriptome after retinal injury. We can now examine the transcriptome data from injured retina by using not only various injury models [Vazquez et al., 2004; Yoshimura et al., 2003; Ahmed et al., 2004; Wilson et al., 2003; Chen et al., 2004] but also combining data from different regions of the central nervous system (CNS) [Matzilevich et al., 2002; Dash et al., 2004; Di Giovanni et al., 2003; Chesler et al., 2005].

Our previous analysis of rat retina identified three major transcript phases after injury: an early acute phase (within hours), an intermediate subacute phase (within 1 to 2 days), and a late chronic phase (within 3 days to weeks; [Vazquez et al., 2004]). The early acute phase is characterized by the transient upregulation of immediate-early genes, mainly transcription factors and activating cytokines. The intermediate subacute phase is characterized by the expression of cellcycle and cell-death genes. The late chronic phase is characterized by the expression of genes involved in neuronal and glial structural remodeling. Similar changes were described in injury models in the rat retina [Yoshimura et al., 2003; Ahmed et al., 2004; Wilson et al., 2003; Chen et al., 2004], brain [Matzilevich et al., 2002; Dash et al., 2004], and spinal cord [Di Giovanni et al., 2003]. For example, the early acute genes Fos, Jun, Egrl, and Nfkbl, as well as the late chronic genes Gfap, A2m, Apoe, and $H s p 27$, display transcript changes resulting from virtually any trauma or stress throughout the CNS [Vazquez et al., 2004; Yoshimura et al., 2003; Ahmed et al., 2004; Wilson et al., 2003; Chen et al., 2004; Matzilevich et al., 2002; Dash et al., 2004; Di Gio- 
vanni et al., 2003]. The similarities of coordinate changes in the retina, brain, and spinal cord indicate the presence of common transcriptional networks controlling the CNS response to injury.

GeneNetwork (www.genenetwork.org, [Chesler et al., 2005; Bystrykh et al., 2005]) is a powerful analytical tool to define transcriptional networks and regulators in CNS tissues. At the heart of this analysis is a unique population, the recombinant inbred (RI) strains of mice. One group of mice were derived by inbreeding for over 20 generations the F1 crosses between C57BL/6 (B) and DBA/2J (D) mouse strains. Each BXD RI strain has a shuffled genome consisting of a unique recombination of the C57BL/6 and DBA/2J genomes, forming a segregating population of mice. These RI mouse strains, the associated microarray databases, and the geneticanalysis tools at GeneNetwork allow one to explore regulatory loci and networks built around any transcript [Chesler et al., 2005; Bystrykh et al., 2005]. The best way to think about the genetic linkage mapping is at the transcript level. If there is specific variability in transcript abundance among members of a segregating population, then it is possible to map transcript abundance to a specific chromosomal locus (for methodology, see [Chesler et al., 2005; Bystrykh et al., 2005]). One example of this type of analysis was presented by Chesler and colleagues [Chesler et al., 2005] where a group of genes involved in synaptic vesicle regulation forms a network with distinct regulatory loci. The concept of genetic analysis of transcript data is called "expression genetics" or "genetical genomics" (for an overview see [Broman, 2005]). Of interest to the vision community is the comprehensive analysis of regulatory loci controlling gene expression of the mouse brain. Both retina and brain are tissues derived from the neural tube. Thus, they share similar cell types with similar transcriptional regulation. For example, the transcription factor STAT3 plays an important role in activating cortical astrocytes [Acarin et al., 2001] and retinal Muller glial cells [Wang et al., 2002]. Thus, it may be possible to use available genetic analyses of brain transcript data to define genetic networks and regulatory loci for the retina. To identify genomic loci and potential candidate genes controlling changes in the rat retina, we turned to our publicly available database. The GeneNetwork database was generated using RI mouse strains and a similar data set is not currently available for the rat. Since the rat is closely 
related to the mouse and since transcriptional expression in the normal and injured CNS is highly conserved across rodent species [Natale et al., 2003], we have chosen to use this mouse database to further analyze the changes in gene expression that occur following injury to the rat CNS.

Here we describe a method to define regulators of retinal wound healing that uses published and publicly available microarray data, transcript regulatory loci databases, and bioinformatic resources. As a starting point, we define regulators that control the expression of acute phase genes after a mechanical injury to the rat retina [Vazquez et al., 2004]. First to eliminate retina-specific genes and to increase the power of our analysis, we extract a group of acute phase genes that are common to injured rat retina, brain, and spinal cord. Second, we determine the regulatory loci controlling the expression of acute phase genes in mouse brains using GeneNetwork. Third, we use computational tools to predict the candidate upstream regulator. This unique combination of analytical tools provides, for the first time, an analysis of retinal microarray data that predicts genetic networks and their candidate upstream regulators.

\section{Methods}

\section{Meta-analyses of Microarray Data Sets}

We performed meta-analyses of previous experimental data (Table 3.1) to select a group of candidate genes for a common response to injury in neural tissues. We previously published CNS microarray analyses that examined the transcriptome response of injured rat retina and brain, the transcriptome of cultured human and rat astrocytes, and the regulatory loci controlling transcript expression in the mouse brain [Vazquez et al., 2004; Matzilevich et al., 2002; Dash et al., 2004; Chesler et al., 2004; Yang et al., 2004]. To complement our analysis of reactive CNS genes, we obtained publicly available data that examined the transcriptome response of injured rat spinal cord, the transcriptome of developing mouse retina, as well as the transcriptome of cultured mouse neurons, mouse astrocytes, and rat microglia [Di Giovanni et al., 2003; Duker et al., 
Table 3.1. Data used to define acute phase genes.

\begin{tabular}{|c|c|c|c|c|c|}
\hline Tissue & $\begin{array}{c}\text { Reactive response } \\
\text { inducer }\end{array}$ & Species & $\begin{array}{l}\text { Microarray } \\
\text { Platform }\end{array}$ & Experimental conditions & GEO number or reference \\
\hline Injured retina & Retinal tear & Rat & U34A & $4 \mathrm{~h}$; and $1,3,7, \& 30 \mathrm{~d}$ & Vazquez et al., 2004 \\
\hline Injured brain & Cortical impact & Rat & $\mathrm{U} 34 \mathrm{~A}$ & $3 \mathrm{~h}$ & Matzilevich et al., 2002 \\
\hline Injured spinal cord & Cord contusion & Rat & $\mathrm{U} 34 \mathrm{~A}$ & $4 \mathrm{~h}$ & Di Giovanni et al., 2003 \\
\hline Optic nerve head astrocyte & Cell culture & Human & U95Av2 & Cell culture & Yang et al., 2004 \\
\hline Cortical astrocyte & Cell culture & Rat & U34A & Cell culture & GSM34300 \\
\hline Brain microglia & Cell culture & Rat & U34A & Cell culture & Duke et al., 2004 \\
\hline Neuron & Cell culture & Mouse & U74Av2 & Cell culture & Kraft et al., 2004 \\
\hline Developing retina & Development & Mouse & U74Av2 & P0, P4, P8, P10, P12, P14,P21, \& adult & Dorell et al., 2004 \\
\hline Adult forebrain & Genetic variation & Mouse & U74Av2 & C57BL/6, DBA2/J, F1, \& 32 BXD RI & Chesler et al., 2005 \\
\hline
\end{tabular}

We performed a meta-analysis of previous experimental data to select a group of acute phase genes that are part of a common response to injury in neural tissues. Previously, we examined transcript levels in injured retina, injured brain, cultured astrocytes, and in forebrains from a panel of 35 BXD RI strains [Vazquez et al., 2004; Matzilevich et al., 2002; Dash et al., 2004; Chesler et al., 2004; Yang et al., 2004]. Microarray data sets describing transcript levels in injured spinal cord, cultured microglia, cultured neurons, and developing retina were obtained from publicly available published studies [Di Giovanni et al., 2003; Duker et al., 2004; Moran et al., 2004; Kraft et al., 2004; Dorell et al., 2004]. All datasets are available online either at Gene Expression Omnibus (GEO,www.ncbi.nlm.nih.gov/geo) or at the author's or journal's website. 
2004; Moran et al., 2004; Kraft et al., 2004; Dorell et al., 2004]. Table 3.1 makes clear the source and availability of each microarray data set. The use of microarray datasets involved three different meta-analyses. First, a group of acute phase genes commonly expressed throughout the CNS was defined by directly comparing microarray data from injured rat retina, brain, and spinal cord. For this analysis, we obtained the raw data (CEL files), determined signal values using Microarray Suite 5.0 (MAS 5.0; Affymetrix, Santa Clara, CA), transformed signals to a log scale (base 2), and normalized microarray mean intensity to 8 as described previously [Vazquez et al., 2004]. These transformations yielded signal intensities ranging from 1 to 18 relative units of fluorescence. Further analyses included only transcripts with medium to high abundance (that is, signals greater than 8.64 [Vazquez et al., 2004]). To determine significant differences, we used Student's t-test $(\mathrm{P}<0.05)$ and changes greater than 2 -fold. The second meta-analysis determined if an acute phase gene was expressed in cultured astrocytes, cultured microglia, and cultured neurons, as well as in the developing retina. An acute gene was considered present in a tissue if it displayed

medium to high abundance [Vazquez et al., 2004]. In the third meta-analysis, we used GeneNetwork to examine transcript regulation in mouse forebrains. Transcriptome analyses with Affymetrix RG-U34A and MG-U74Av2 survey about nine and twelve thousand targets (about 30 and $40 \%$ of the rodent transcriptome; [Affymetrix NetAffex, June 2005 Annotation]).

\section{Online Regulatory Locus Analyses}

To define regulatory loci that control gene expression in mouse brain, we used GeneNetwork [www.genenetwork.org], which is maintained by members of our group (E.J.C, L.L, K.F.M, and R.W.W). Details on the methods, data, and analyses are available at GeneNetwork [Chesler et al., 2005; Bystrykh et al., 2005]. Briefly, GeneNetwork is a suite of databases and analysis software that identifies regulatory loci that control transcript abundance of nearly 12,000 genes in a panel of 32 BXD RI mouse strains derived from C57BL/6 (B) and DBA/2J (D) mice, both parental strains, and the F1 hybrid (a total of 35 isogenic lines [Chesler et al., 2005; Bystrykh et al., 
2005]). Strain means for transcript abundance were generated from three to five Affymetrix U74Av2 microarrays, and each individual array was hybridized to a pool of forebrain tissue taken from three adult animals [Chesler et al., 2005]. To maximize strain-dependent expression variance or heritability, probe-set signals were measured by weighting the perfect-match (PM) probes by their heritability [Manly et al., 2005]. Using quantitative genetic analysis and a genetic map consisting of 779 fully genotyped markers, GeneNetwork correlates the mean abundance of each transcript to genotypes at locations throughout the mouse genome. The average distance between adjacent markers is approximately 4 megabases $(\mathrm{Mb})$. Here, we examined the regulation of acute phase genes in mouse brains using GeneNetwork and the weighted-by-heritability transforms [UTHSC Brain mRNA U74Av2 HWT1PM, December 2003]. Variability across strains was measured using analysis of variance (ANOVA) testing the between-strain variance compared with the total variance for 100 arrays from 35 mouse strains. The degrees of freedom for the between-group and total variance are 34 and 99. Strain-specific variation is significant $(\mathrm{p}<0.05)$ when $\mathrm{F}_{34,99}>1.5$. Whole genome maps for all transcripts are replicated and recomputed using a variety of transforms and analytical methods in GeneNetwork, including simple and composite interval mapping, multiple-trait clustering, and principal component analysis. We also confirmed the strength of a candidate upstream regulator using the cerebellum GeneNetwork database [SJUT Cerebellum mRNA M430 MAS5, January 2004]. Since gene names may differ across species and multiple probe sets may be available in the U74Av2 microarray, we include the probe set identifier when referring to genes analyzed with GeneNetwork. We confirmed gene identity of a probe set using Affymetrix NetAffex [June 2005 Annotation] and Ensembl AffyProbe [v33] databases.

\section{Online Bioinformatic Resources}

To identify candidate genes within a regulatory locus, we located genes within the appropriate genomic interval using the Genome Browser [Mouse May 2004 assembly]. Single nucleotide 
polymorphisms (SNPs) were identified by comparing genome sequences in the Celera SNP (Celera Discovery Systems, CA; [July 2003 Assembly]), Ensembl Mouse SNPView [v33], and Entrez SNP databases. Once the SNPs were identified, a computational approach was used to determine if the SNP within the $I d 2$ promoter (rs4229289, for sequence variation and genomic context see Ensembl Mouse SNPView) was within a transcription factor binding site. First, we determined whether the rs4229289 SNP was located within a highly conserved region using Genome Browser Conservation tool [Mouse May 2004 Assembly]. Second, we obtained a highly conserved sequence around the rs4229289 SNP from the alignment of mouse, rat, and human sequences using Genome Browser Conservation tool [May 2004 Assembly]. Alignments with other species were not available. Third, we located putative transcription factor binding sites within the conserved region using the vertebrate TRANSFAC database available through MOTIF database and using the default cut-off score [Knuppel et al., 1994].

Several online databases provide transcript data or transcript distribution in the CNS. Gene Expression Omnibus (GEO) is a high-throughput gene expression abundance data repository, as well as a curated, online resource for gene expression data browsing, query, and retrieval [www.ncbi.nlm.nih.gov/geo]. Retina Developmental Gene Expression describes the gene expression profile of thousands of genes in the developing postnatal mouse retina that were analyzed by hybridization to Affymetrix Mu74 Av2 [www.scripps.edu/cb/friedlander/gene_expression/; Dorell et al., 2004]. Mouse Retina SAGE Library provides serial analysis of gene expression tags or in situ hybridization images for a limited number of transcripts expressed in the developing and adult mouse retina [https://bricweb.partners.org/cepko/default.asp; Blackshaw et al., 2004]. Gene Expression Nervous System Atlas (GENSAT) maps the transcript distribution in the mouse CNS using in situ hybridization [www.ncbi.nlm.nih.gov/gensat]. 


\section{Results}

\section{Acute Phase Genes}

In our continuing efforts to understand the retinal response to injury, we concentrated on the transcriptome changes that occur within hours after retinal injury: the acute phase genes. We know that there is a sequence of changes leading to reactive gliosis and remodeling of the retina [Vazquez et al., 2004]. To make our analysis more robust and generalized for CNS injury, we combined our injured rat retina data with results from injured rat brain and spinal cord. In these three data sets, 30 genes demonstrated a significant change in expression level (Table $3.2 ; P<$ 0.05; change $>2$-fold; 4 h postinjury). All of the common changes in the three data sets were upregulated after injury. No common down-regulated transcripts were observed in injured retina, brain, and spinal cord. These acute phase genes can be expressed in a wide range of neural cells including astrocytes, microglia, and neurons (Table 3.2). These results suggested a generalized group of transcript changes that occurs in the CNS after injury.

The acute phase genes belong to several transcript categories including transcription, cytokines, cell adhesion, extracellular proteases, and metabolism. The major transcript group $(46.6 \%)$ was related to the regulation of gene expression including transcription factors and transcription modulators. Based on gene expression dynamics, acute phase genes displayed either transient or chronic expression changes after retinal injury (Table 3.2). For example, transcription factors such as Crem, Egrl, Fos, Fosl1, Junb, Egr1, Ier3, Btg2, Atf3, and Nr4al were upregulated, with a transient surge at $4 \mathrm{~h}$. Other transcription factors such as Irf1, Stat3, Nfkb1, Id2, and Cebpd displayed an upregulation at $4 \mathrm{~h}$, followed by a smaller, but sustained, expression out to 30 days after retinal injury (Table 3.2). 
Table 3.2. Acute phase genes expressed in the injured CNS.

\begin{tabular}{|c|c|c|c|c|c|}
\hline Symbol & Name (alias) & $\begin{array}{l}\text { Astrocytic } \\
\text { Expression }\end{array}$ & $\begin{array}{l}\text { Microglial } \\
\text { Expression }\end{array}$ & $\begin{array}{c}\text { Neuronal } \\
\text { Expression }\end{array}$ & $\begin{array}{c}\text { Chronic } \\
\text { Expression }\end{array}$ \\
\hline \multicolumn{6}{|l|}{ Transcription } \\
\hline Atf3 & Activating transcription factor 3 & + & + & - & - \\
\hline Btg2 & B-cell translocation gene 2 & + & - & + & - \\
\hline Crem & cAMP responsive element modulator & - & - & - & - \\
\hline Egrl & Early growth response 1 (Krox-24/NGFI-A) & + & - & + & - \\
\hline Fos & FBJ osteosarcoma oncogene & + & + & + & - \\
\hline Fosll & Fos-like 1 & + & - & - & - \\
\hline Junb & Jun-B oncogene & + & - & + & - \\
\hline Ier3 & Immediate early response 3 (PRG1) & + & + & + & - \\
\hline Nr4al & Nuclear receptor subfamily 4a1 (NGFI-B/nurr7) & + & - & + & - \\
\hline Cebpd & CCAAT/enhancer binding protein delta & + & - & + & + \\
\hline $\operatorname{Id} 2$ & Inhibitor of DNA binding 2 (Idb2) & + & + & + & + \\
\hline Irf1 & Interferon regulatory factor 1 & + & + & - & + \\
\hline Nfkbl & Nuclear factor kappa B p105 & + & + & - & + \\
\hline Stat3 & Signal transducer and activator of transcription 3 & + & - & + & + \\
\hline \multicolumn{6}{|l|}{ Cytokine } \\
\hline Cxcl10 & Chemokine (C-X-C motif) ligand 10 & + & + & - & + \\
\hline Illa & Interleukin 1 alpha & - & + & - & - \\
\hline$I l l b$ & Interleukin 1 , beta & - & + & - & + \\
\hline Scya2 & Small inducible cytokine A2 (Ccl2) & - & + & - & - \\
\hline Scya3 & Small inducible cytokine A3 (Ccl3) & + & - & - & - \\
\hline \multicolumn{6}{|c|}{ Cell adhesion/ECM } \\
\hline Cd44 & Cd44 antigen & + & + & - & + \\
\hline Icam 1 & intercellular adhesion molecule-1 & + & + & - & + \\
\hline Plat & Plasminogen activator tissue & + & + & - & + \\
\hline Serpinel & Serine proteinase inhibitor-1 (Pai1) & + & - & - & + \\
\hline Timpl & Tissue inhibitor of metalloproteinase-1 & + & + & - & + \\
\hline \multicolumn{6}{|l|}{ Metabolism } \\
\hline Hmoxl & Heme oxygenase & + & + & + & + \\
\hline Mat $2 a$ & Methionine adenosyltransferase IIa & + & - & - & - \\
\hline Ptgs 2 & Prostaglandin synthase 2 (Cox-2) & + & - & - & - \\
\hline \multicolumn{6}{|l|}{ Other } \\
\hline Ptpn16 & Tyrosine phosphatase non-receptor type 16 (Dusp1/Ptp) & + & - & + & - \\
\hline Adfp & Adipose differentiation related protein & + & + & - & - \\
\hline Ifrdl & interferon-related developmental regulator 1 & + & - & + & - \\
\hline
\end{tabular}

Acute phase genes displayed transcript changes in injured retina, brain, and spinal cord. Astrocytic, microglial, and neuronal expressions refer to moderate to high transcript levels in cultured astrocytes [Yang et al., 2004; Kraft et al., 2004], microglia [Duker et al., 2004; Moran et al., 2004], neurons [Kraft et al., 2004]. Chronic expression describes expression in rat retina from 3 to 30 days after injury [GEO data set GSE1001; Vazquez et al., 2004]. 


\section{Regulatory Locus Analyses}

The grouping of genes with similar transcript expression changes suggested the presence of common upstream modulators. To begin defining regulatory mechanisms controlling the retinal injury response, we tested the hypothesis that acute phase genes share common regulatory loci (Figure 3.1). Transcriptome-wide regulatory loci for the mouse brain were mapped previously by our group [Chesler et al., 2005] and can be replicated and recomputed using the databases and genetic-analysis tools at GeneNetwork. We used the mouse brain transcriptome and genetic analysis at GeneNetwork to define common regulatory loci for acute phase genes.

A genome-wide scan estimates the association of transcript abundance variability against genetic markers across the mouse genome. For example, the abundance variability of $I d 2$ was high in brains from BXD RI mouse strains $\left(\mathrm{F}_{34,99}=5.2\right.$; probe set 93013_at for $\left.I d 2 \mathrm{mRNA}\right)$. In the genome-wide scan (Figure 3.1A), the correlation of $I d 2$ variability across the mouse genome is indicated by the likelihood ratio statistic curve (LRS, blue line). At chromosome 12, 10-30 Mb distal to the centromere, Id2 variation correlated highly with genetic markers D12Mit209, D12Nyu7, Rrm2, D12Mit234, and D12Mit242 (Figure 3.1A; LRS = 44). In a second example, Fos displayed variation $\left(\mathrm{F}_{34,99}=1.5\right.$; probe set 160901_at) that also correlated with genetic markers that span a region of chromosome 12 at 10-30 Mb (Figure 3.1A, LRS = 13). The correlation of Fos and $I d 2$ expression to a chromosomal interval on chromosome 12 suggested that this locus contains one or more polymorphic genes that affect the levels of both transcripts.

In the group of acute phase genes, a number of transcripts had a common regulatory locus on chromosome 12. These included Fos, Nr4al, Id2, Egrl, Crem, Junb, Ccl3, and Ptpn16, all of which shared a strong correlation to the locus on chromosome 12, 10-30 Mb (Figure 3.1B; LRS > 10; probe sets 160901_at, 102371_at, 93013_at, 98579_at, 160526_at, 102362_at, 102424_at, and 104598_at, respectively). By aligning genome-wide scans, we identified a strong band of regulatory loci (Figure 3.1B). Most of the genes (Fos, Nr4al, Egrl, Crem, Junb, Ccl3, and Ptpn16) regulated by this genomic location are found outside the regulatory locus, termed 


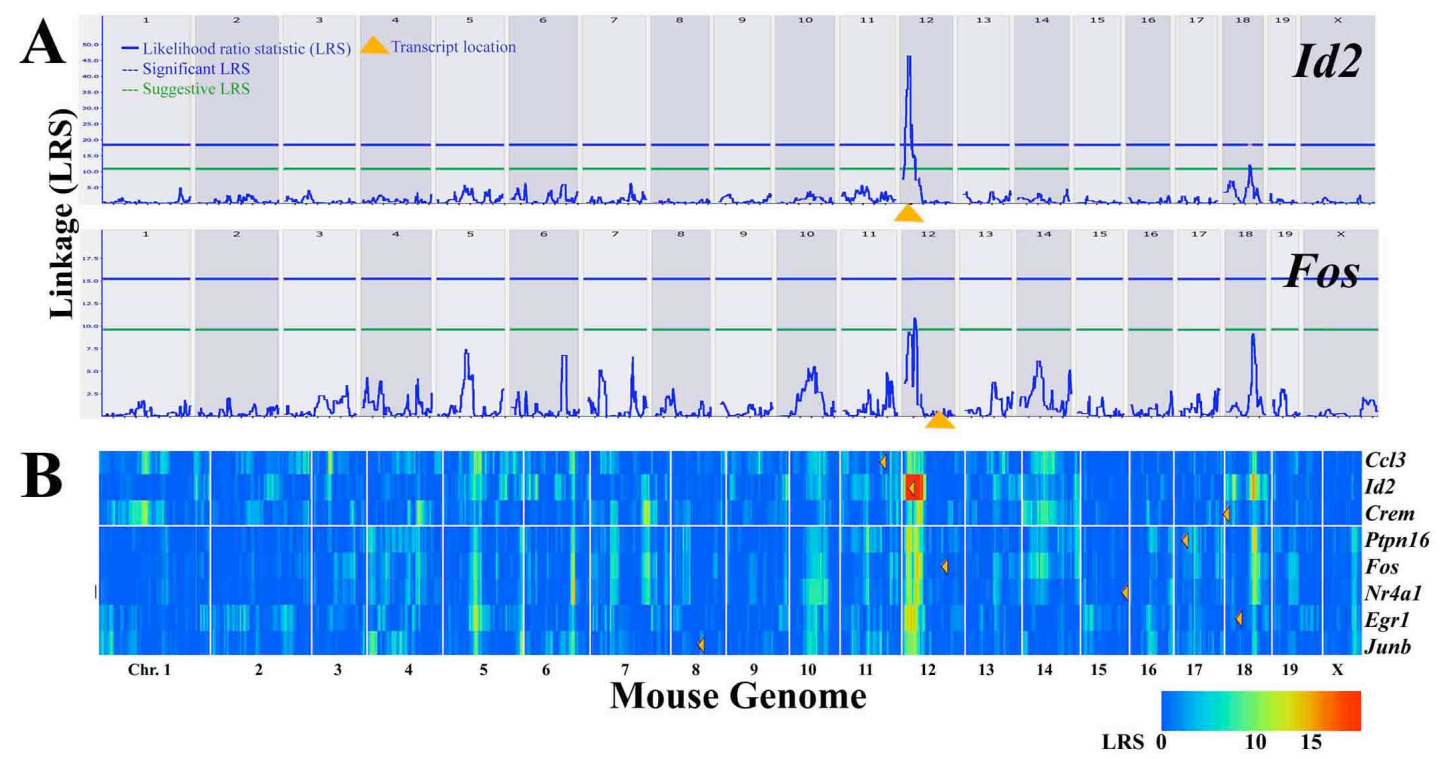

Figure 3.1. Acute phase genes share regulatory loci. We used the genetic analysis of transcript expression at GeneNetwork [www.genenetwork.org] to define genomic loci that control transcript abundance variability in mouse brains. At the heart of this analysis is a unique population, the recombinant inbred (RI) strains of mice. One group of mice were derived by inbreeding for over 20 generations the F1 crosses between C57BL/6 (B) and DBA/2J (D) mouse strains. Each BXD RI strain has a shuffled genome consisting of a unique recombination of the C57BL/6 and DBA/2J genomes, forming a segregating population. These RI mouse strains, the associated microarray databases, and the genetic-analysis tools at GeneNetwork allow one to explore regulatory loci and networks built around any transcript [Chesler et al., 2005; Bystrykh et al., 2005]. The best way to think about the genetic linkage mapping is at the transcript level. If there is specific variability in transcript abundance among members of a segregating population, then it is possible to map transcript abundance to a specific chromosomal locus. Genetic linkage maps (AC) show that a group of acute phase genes is controlled by a regulatory locus on chromosome 12 . A: Individual genome-wide maps for $I d 2$ and Fos display linkage of transcript variability (y-axis) to loci across the mouse genome (x-axis). Genetic analysis of transcript data from brain tissue was determined in a panel of BXD RI mouse strains [Chesler et al., 2005]. Maps were generated by linking transcript variability against 779 genetic markers that are interspaced along the mouse genome by an average of 4 Megabase pairs (Mb). The alternating white and blue columns represent chromosomes. The linkage between transcript variation and genetic differences at a particular genetic locus is measured in terms of likelihood ratio statistic (LRS, solid blue line). Dashed horizontal lines mark transcript-specific significance thresholds for genome-wide $\mathrm{P}<0.05$ (significant, blue) and genome-wide $\mathrm{P}<0.63$ (suggestive, green). Orange triangle indicates gene location. B: Multiple parallel genome-wide maps display linkage using color hues with yellow and red bands representing suggestive and significant LRS. A common regulatory locus on chromosome 12 is identified for selected acute phase genes. Within this locus, there is a genomic element modulating the expression of acute phase genes such as Fos, Junb, and Egrl. Individual and multiple genome-wide maps were generated using tools at GeneNetwork. Orange triangles indicate gene location. Linkage maps were generated using the Interval Mapping and Cluster Tree tools at GeneNetwork [UTHSC Brain mRNA U74Av2 HWT1PM, December 2003]. 
"trans-regulatory locus." One of the genes, $I d 2$, lies within the regulatory locus (orange arrowhead, chromosome 12 at $20.1 \mathrm{Mb}$; Figure 3.1A and 3.1B). This is termed a "cis-regulatory locus" for Id2. Transcripts genetically linked to the same regulatory locus are part of a genetic network [Chesler et al., 2005]. In this case, Fos, Nr4al, Id2, Egr1, Crem, Junb, Ccl3, and PtpnI6 are part of a genetic network that is controlled by the regulatory locus on chromosome $12,10-30 \mathrm{Mb}$. In addition to the regulatory locus on chromosome 12, we identified two additional loci that may regulate transcript levels of acute phase genes (Figure 3.2). These regulatory loci were located on chromosomes 6 and 14. In some cases, transcripts correlated to multiple regulatory loci. For example, Fos and Nr4al were genetically linked to regulatory loci on chromosomes 6 and 12, whereas, $N f k b 1$ and $S c y a 2$ correlated with regulatory loci on chromosomes 6 and 14. The genetic relationships between transcript variation and genomic loci defined three genetic networks controlling the expression of acute phase genes in mouse forebrains (Figure 3.2). The regulators for these genetic networks are located within the regulatory loci on chromosomes 6,12 , and 14 .

\section{Evaluating Candidate Upstream Regulators}

Once a regulatory locus was identified, the third step in our analysis refined the genomic location responsible for the mapping to this chromosomal interval. Within each regulatory locus, a genomic element generates the variation in expression of acute phase genes. Ultimately, the genomic differences between the C57BL/6 and DBA/2J mice underlie the transcript abundance variability observed in BXD RI mouse strains. Since the BXD RI mouse strain haplotypes are finely mapped and the parental genomes are sequenced, we have thorough SNP maps for the 35 strains [Chesler et al., 2005]. SNPs affecting the expression of acute phase genes may be found in a number of genomic elements including promoters, enhancers, exons, or introns. These genomic elements may affect the transcript levels of the upstream regulator. Hundreds or even thousands of SNPs may lie within a regulatory locus. Simple rules help select a candidate gene. A candidate gene lies within the regulatory locus, displays SNPs, and is functionally relevant to the genes correlating to the regulatory locus. Biological significance of SNPs can be queried using 


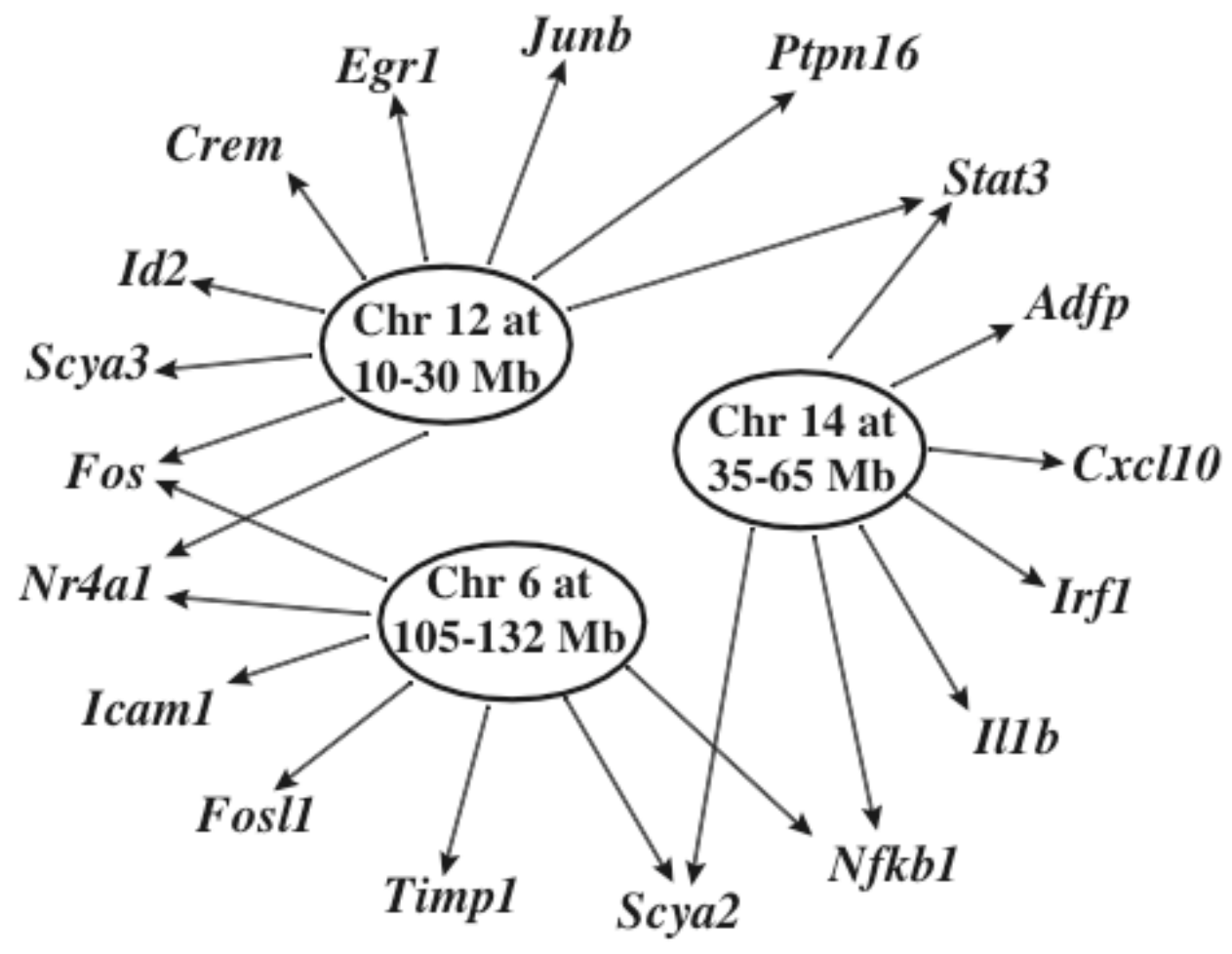

Figure 3.2. Genetic networks controlling acute phase transcripts. Genetic networks were derived from transcripts sharing regulatory loci in mouse brains. As shown in Figure 3.1, acute phase transcripts Fos, Egr1, Nr4a1, Junb, Stat3, Id2, Crem, Ptpn16, and Scay3 are controlled by a genomic locus on chromosome 12. We also showed in Table 3.2 that these transcripts are part of a common phase response to injury in retina, brain, and spinal cord. Transcripts genetically linked to the same regulatory loci and highly regulated in injured CNS are hypothesized to be part of a genetic network. In this diagram, lines connecting specific genes to loci represent a correlation between transcript expression and the regulatory locus. The diagram also illustrates two networks controlled by genomic loci on chromosomes 6 and 14 . 
programs that predict whether SNPs affect known DNA binding sites or protein sequence motifs [Knuppel et al., 1994]. The vast biological content within online resources provides a powerful tool to identify the regulatory element controlling the expression of genes within a biological pathway, in our case the response of the transcriptome to retinal injury. We present our analysis of candidate regulators for several of the loci. We conclude with evidence for a strong candidate gene for the regulatory locus on chromosome 12 .

The regulatory locus on chromosome $14,35-65 \mathrm{Mb}$, modulates the transcript variability of transcription factors Stat3, Nfkb1, and IrfI; cytokines Illb and Scya2; and development-related gene Adfp (Figure 3.3A; probe sets 102736_at, 103486_at, 161281_f_at, 161443_r_at, 93858_at, 98427_s_at, and 99099_at). This is a trans-regulatory locus for these genes, as they all lie outside this locus. A genomic element within the locus is a trans-regulator for these acute phase genes. Within this locus, there were 315 positional candidate genes (Figure 3.3B and 3.3C). A simple procedure to weigh the relevance of a positional gene is to determine whether it displays significant transcript variability in BXD RI mouse strains and whether its variability maps to its gene location - that is, does the gene display a cis-regulatory locus? Highly variable transcripts included Rnase 4, AW045965, M6a, Boct, Dad1, Ctsb, Cnih, and Ndrg2 ( $\left.\mathrm{F}_{34,99}>4\right)$. Of these, Rnase4, AW045965, M6a, Boct, Boct, Cnih, and Ndrg2 linked strongly to the regulatory locus on chromosome 14 (LRSs > 17; Figure 3.3D, data for Ndrg2 shown, probe set 96088_at). To further evaluate the role of these positional genes, we determined whether their self-regulatory role extended to the cerebellum of BXD RI mouse strains. Rnase 4, AW045965, M6a, and Ndrg2 displayed significant cis-regulatory loci on chromosome 14, 35-65 Mb (LRSs > 12, data not shown; probe sets 96038_at, 100073_at, 104456_at, and 161610_at). These data suggested that the regulatory role of the locus on chromosome 14 is conserved across CNS tissues. Rnase4, AW045965, $M 6 a$, and $N d r g 2$ are candidate upstream regulators because they display cis-regulatory loci. 
Figure 3.3. Evaluating candidate genes within chromosome 14 locus. A: The transcript variability of Adfp, Scya2, Irfl, Nfkb1, Illb, Stat3, and CxcllO in brains from BXD RI mouse strains is genetically linked to a regulatory locus on chromosome 14. This network was generated by comparing genome-wide scans that measure the linkage of transcript variability across the mouse genome, as described in Figure 3.1. These transcripts are also upregulated as acute phase transcripts in retina, brain and spinal cord (Table 3.2). Transcripts genetically linked to the same regulatory loci and highly regulated in injured CNS are hypothesized to be part of a genetic network. B: The combined genome-wide map shows that their transcript expression has a strong genetic linkage to a locus within chromosome 14 . The genome wide scan, zoomed at chromosome 14, shows the genetic linkage (y-axis, likelihood ratio statistic [LRS]) across the chromosome (x-axis, Megabase pairs [Mb]). The locus (35 to $65 \mathrm{Mb}$ ) includes 315 genes, some of which have a high or low single nucleotide polymorphism (SNP) density. SNP density is denoted by the height of the orange lines on the $\mathrm{x}$-axis. Several rules may help in selecting candidate gene responsible for this regulatory locus. The first rule is there have to be SNPs present within the coding or regulatory region of the gene of interest. As a first approximation we used SNP density that is displayed at the bottom of panel $\mathbf{B}$ and $\mathbf{D}$. The second rule is a high degree of transcript abundance variability in the BXD RI strains (illustrated in C). C: Transcript abundance variability in normal forebrains of BXD RI mouse strains is due to genetic polymorphisms between the parental C57BL/6 and DBA/2J mouse strains. The graph illustrates transcript abundance variability (yaxis) for genes (dots) within the 35 to $65 \mathrm{Mb}$ interval of chromosome 14 (x-axis). For a transcript to be mapped its abundance must vary across the BXD RI mouse strains. The higher the variation in transcript abundance the more likely that the gene is a candidate. Furthermore we expect that the variability is due to a polymorphism in the candidate gene, suggesting that the polymorphic gene is modulating its own expression level (that is, a "cis-regulatory locus"). We measured transcript variability using an analysis of variance (ANOVA) that tests the between-strain variance compared with the total variance for 100 arrays from 35 mouse strains. The degrees of freedom for the between-group and total variance are 34 and 99. Strain-specific variation is significant ( $p$ $<0.05$ ) when $\mathrm{F}_{34,99}>1.5$. In the graph, each dot represents a gene with its variability measured by the ANOVA F-statistic (y-axis) and its genomic location within chromosome 14 described in Mb. Rnase4, AW045965, M6a, Boct, Dad1, Ctsb, Cnih, and Ndrg2 are polymorphic genes that displayed significant transcript variability and cis-regulatory loci in normal forebrains of BXD mouse strains. D: The third criterion identifies the genes that display self regulation across CNS tissues. For example, the transcript variability of $\mathrm{Ndrg} 2$ (red circle in $\mathbf{C}$ ) displayed a cisregulatory locus in brain (LRS=38, probe set 96088_at) and cerebellum (LRS=13, probe set 1448154_at_A) of BXD RI mouse strains. Here we illustrate linkage of Ndrg2 transcript variability in BXD RI mouse strains across chromosome 14. Red triangle indicates gene location. Rnase4, AW045965, and M6a also displayed cis-regulatory loci in brain and cerebellum (data available at GeneNetwork). E: The fourth criterion deals with the biology of the system. Metaanalyses of transcript levels during retinal development and retinal injury can help determine if a candidate gene is a potential regulator in the retina. For example in murine models of retinal development, $\mathrm{Ndrg} 2$ displayed moderate transcript levels as early as postnatal day 4 and was highly expressed in the adult retina. In contrast, Rnase4, AW045965, and M6a were absent from birth through adult stages. Normalized expression data for $N d r g 2$ (y-axis) during murine retinal development (x-axis) is available at Retina Developmental Gene Expression [Dorell et al., 2004]. 

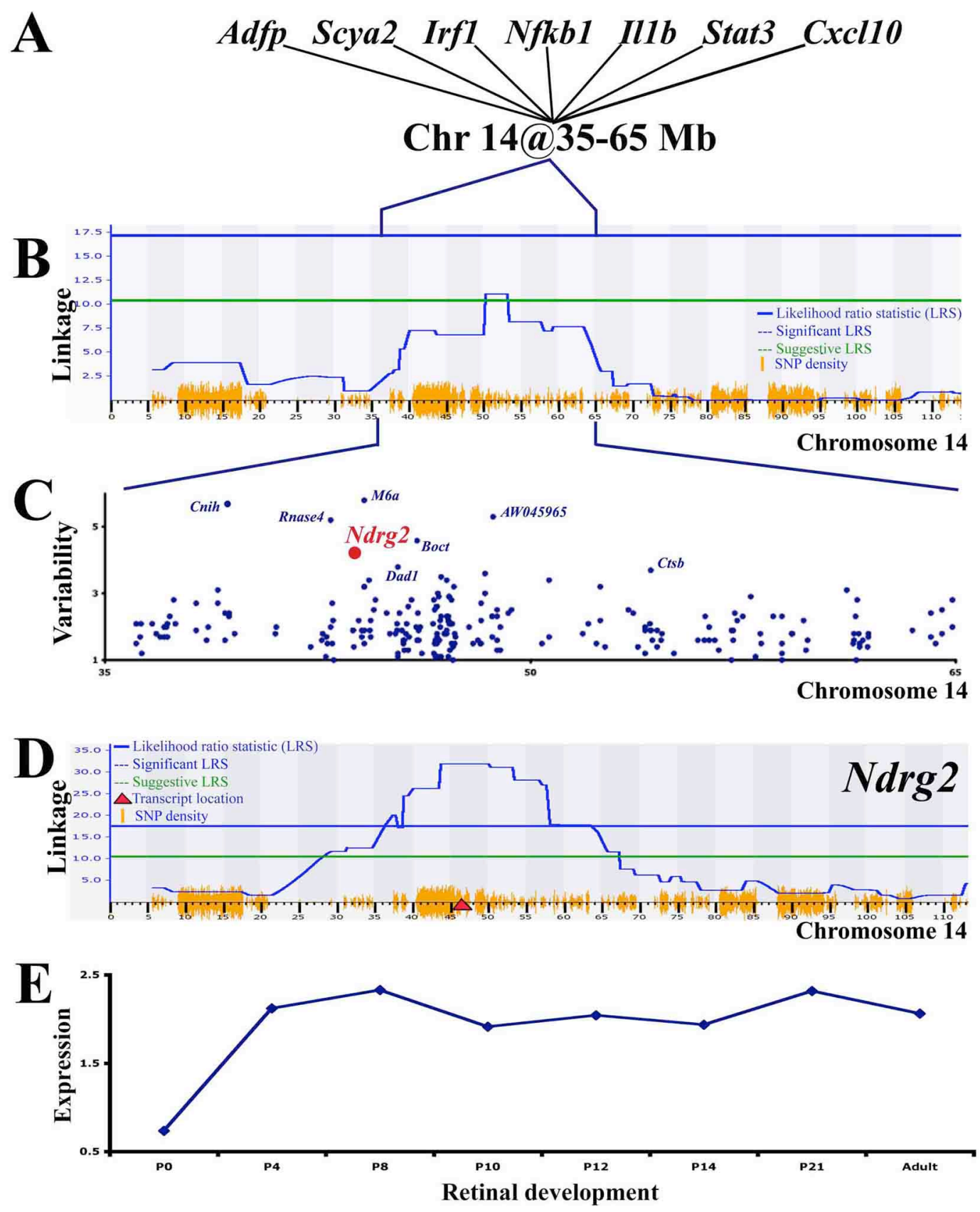
Identifying polymorphic genes also helps to identify the candidate upstream regulator. Sequences for Rnase 4, AW045965, M6a, and Ndrg2 had multiple SNPs between the C57BL/6 and DBA/2J strains. For example, $N d r g 2$ had a high density of SNPs: 7 within the promoter (Celera SNPs IDs mCV23555818, mCV23556083-5, mCV23556089-91), and 25 within exons and introns (Celera SNPs IDs mCV23555752, 756-8, 762-3, 768-70, 774-6, 780-1, 788, 792-4, 804-6, 811-2, 816-7). The presence of SNPs further supported the role of Rnase4, AW045965, $M 6 a$, and Ndrg2 as candidate upstream regulators. The use of transcriptome data from developing mouse retina [Dorell et al., 2004; Blackshaw et al., 2004] or injured rat retina [Vazquez et al., 2004] can be valuable in determining whether a candidate gene plays a role in controlling acute phase genes in the retina. In murine models of retinal development [Dorell et al., 2004; Blackshaw et al., 2004], Ndrg2 displayed moderate transcript levels as early as postnatal day 4 and was expressed in the adult retina (Figure 3.3E; data available at Retina Developmental Gene Expression [Dorell et al., 2004] and Mouse Retina SAGE Library [Blackshaw et al., 2004]). In contrast, Rnase4, AW045965, and M6a were absent from birth through adult retinal stages [data available at Retina Developmental Gene Expression]. Using a suite of computational tools and transcriptome data from CNS gene regulation and development, we defined Ndrg2 ( -myc downstream regulated gene 2) as a positional candidate that displayed a cis-regulatory locus in the mouse brain and cerebellum and that has a high SNP density. At the present time, Ndrg2 is the best candidate gene for the regulatory locus on chromosome 14 and for potentially being a regulator of retinal acute genes. $\mathrm{Ndrg} 2$ displays a conserved self-regulatory role in mouse brain and cerebellum. It is expressed in the developing mouse retina during the critical time of cell proliferation and cell specification. It also displays persistent transcript expression in the adult mouse retina.

The regulatory locus on chromosome 6 at 105-132 Mb also controls the expression of acute phase genes such as Fosl1, Scya2, Nr4al, Fos, Nfkb1, Icam1, and Timpl (Figure 3.4A; probe sets 102371_at, 102736_at, 160901_at, 99835_at, 101141_at, 98427_s_at, and 101464_at). Within the locus, there were 315 positional genes (Figure $3.4 \mathrm{~B}$ ). Using the computational and comparative techniques described above, $C c n d 2$ (Cyclin D2) best met the criteria for a candidate 
Figure 3.4. Evaluating candidate genes within chromosome 6 locus. A: The transcript variability of Scya2, Nfkb1, Fosl1, Timpl, Fos, Nr4al and Icaml in brains from BXD RI mouse strains is genetically linked to the regulatory locus on chromosome 6 . This network was generated by comparing genome-wide scans that measure the linkage of transcript variability across the mouse genome, as described in Figure 3.1. These transcripts are also upregulated as acute phase transcripts in retina, brain and spinal cord (Table 3.2). Transcripts genetically linked to the same regulatory loci and highly regulated in injured CNS are hypothesized to be part of a genetic network. B: The combined genome-wide map shows that their transcript expression has a strong genetic linkage to a locus within chromosome 6,105 to $132 \mathrm{Mb}$. The genome wide scan, zoomed at chromosome 6, shows the genetic linkage (y-axis, likelihood ratio statistic [LRS]) across the chromosome (x-axis, Megabase pairs [Mb]). This locus includes over 200 positional genes, some of which have a high or low single nucleotide polymorphism (SNP) density. SNP density is denoted by the height of the orange lines on the x-axis. Several rules may help in selecting candidate gene responsible for this regulatory locus. The first rule is there have to be SNPs present within the coding or regulatory region of the gene of interest. As a first approximation we used SNP density that is displayed at the bottom of panel $\mathbf{B}$ and $\mathbf{D}$. The second rule is a high degree of transcript abundance variability in the BXD RI strains (illustrated in C). C: Transcript abundance variability in normal forebrains of BXD RI mouse strains is due to genetic polymorphisms between the parental C57BL/6 and DBA/2J mouse strains. The graph illustrates transcript abundance variability (y-axis) for genes (dots) within the 105 to $132 \mathrm{Mb}$ interval of chromosome 6 (xaxis). The higher the variation in transcript abundance the more likely that the gene is a candidate. Furthermore we expect that the variability is due to a polymorphism in the candidate gene, suggesting that the polymorphic gene is modulating its own expression level (that is, a " $\mathrm{cis}$ regulatory locus"). We measured transcript variability using an analysis of variance (ANOVA) that tests the between-strain variance compared with the total variance for 100 arrays from 35 mouse strains. The degrees of freedom for the between-group and total variance are 34 and 99. Strain-specific variation is significant $(\mathrm{p}<0.05)$ when $\mathrm{F}_{34,99}>1.5$. In the graph, each dot represents a gene with its variability measured by the ANOVA F-statistic (y-axis) and its genomic location within chromosome 6 described in Mb. Tulp3, Ccnd2, Tead4, and Atp2b2 are polymorphic genes that displayed significant transcript variability and cis-regulatory loci in normal forebrains of BXD mouse strains. D: The third criterion identifies the genes that display self regulation across CNS tissues. For example, the transcript variability of $C c n d 2$ (red circle in $\mathbf{C}$ ) displays a strong cis-regulatory locus in the forebrain (LRS=25, probe set 97504_at) and cerebellum (LRS=14, probe set probe set 1416122_at_A) of BXD RI mouse strains. Here we illustrate linkage of $C$ cnd 2 transcript variability in BXD RI mouse strains across chromosome 6 . Red triangle indicates gene location. Atp2b2, Tulp3, and Tead4 also displayed cis-regulatory loci in brain and cerebellum (data available at GeneNetwork). The role of a candidate gene as a modulator of the chromosome 6 and wound healing is further strengthen if its transcript is differentially expressed in injured CNS. Ccnd2 displays increased transcript levels in injured rat retina [GEO data set GSE1001, probe set rc_AA899106_at; Vazquez et al., 2004]. 

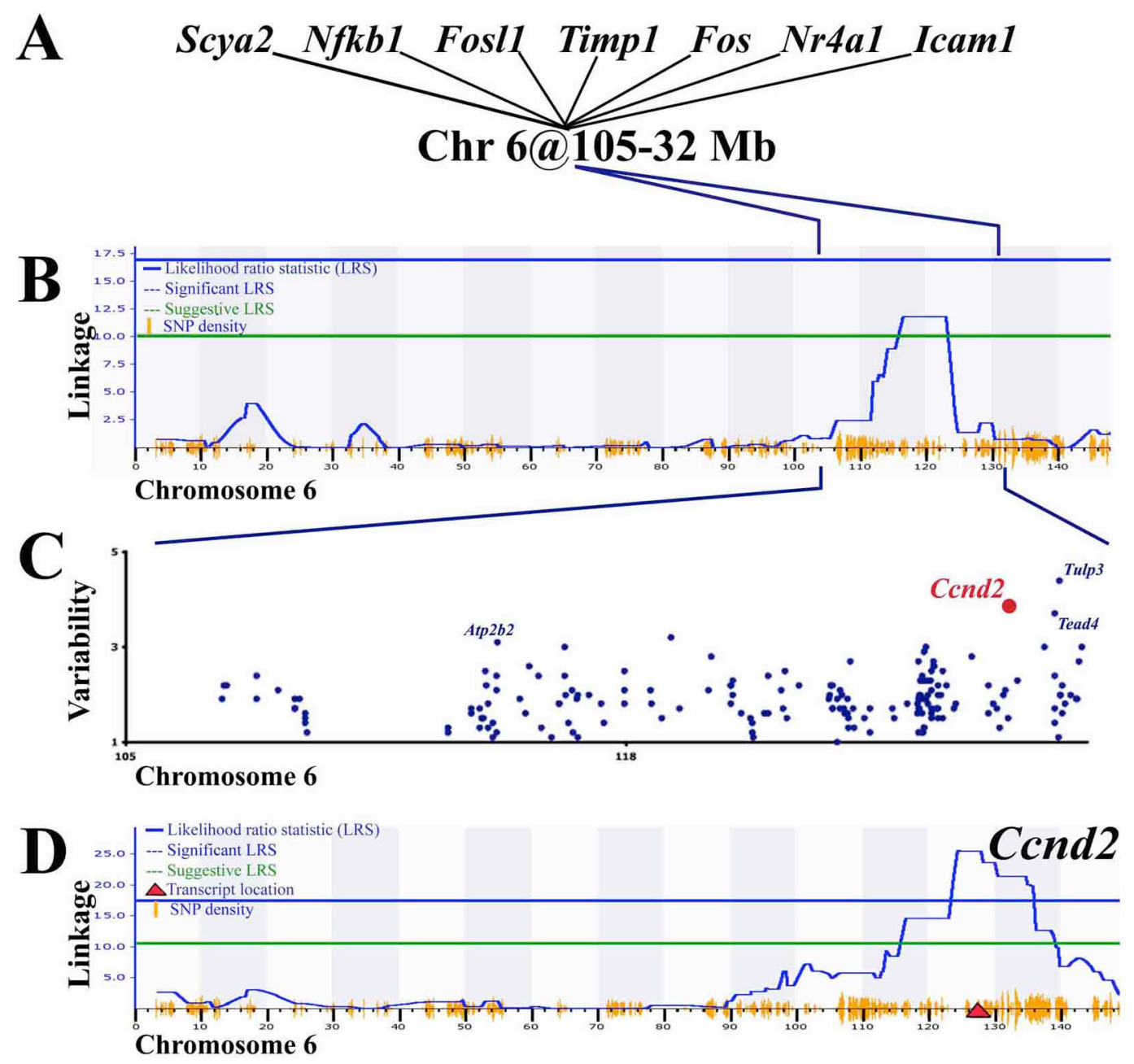
upstream regulator. $C c n d 2$ displayed significant transcript abundance variability $\left(\mathrm{F}_{34,99}=3.8\right.$, Figure 3.4C, probe set 97504_at) and a strong cis-regulatory locus on chromosome 6 (LRS = 29, Figure 3.4D) in brains from BXD RI mouse strains. We also observed a strong cis-regulatory locus for $C c n d 2$ in the cerebellum (LRS = 15; probe set 1416122_at_A; data not shown). Multiple SNPs spanned the transcribed region of Ccnd2 (Celera SNP IDs mCV24153285, 558, 560, 570, $580-2,592-4,615-6)$. These data suggested that $C$ cnd 2 controls the expression of classic acute genes in the CNS of BXD RI mouse strains. In mouse retina, Ccnd2 is expressed during development and adult stages [Mouse Retina SAGE Library, probe set 97504_at; and GENSAT Image 14340]. Moreover, $C c n d 2$ displayed chronic upregulation in the injured rat retina [GEO data set GSE1001, probe set rc_AA899106_at; Vazquez et al., 2004]. Several data defined Ccnd2 as the best current candidate gene for the regulatory locus on chromosome 6 and for potentially being a regulator of retinal reactive genes. It displays conserved self-regulatory role in mouse brain and cerebellum. It is expressed in the developing mouse retina during the critical time of cell proliferation and cell specification. It also displays chronic upregulation in the injured rat retina.

For the network defined by the regulatory locus on chromosome 12 (Figure 3.5A; probe sets 93013_at, 161802_i_at, 161716_at, 160526_s_at, 104598_at, 102424_at, 102371_at, and 102362_i_at), the Inhibitor of DNA binding 2 (Id2) is the best current candidate upstream regulator. First, the positional candidate $I d 2$ (Figure 3.5B; probe set 93013_at) showed significant variability and strong cis-regulatory locus in brains from BXD RI mouse strains $\left(\mathrm{F}_{34,99}=5.2\right.$, Figure 3.5C; and LRS = 44, Figure 3.5D). In the cerebellum, we also observed a strong cisregulatory locus for Id2 (LRS = 13; probe set 1453596_at_A; data not shown). Second, while several cis-regulated genes were located within the regulatory locus on chromosome 12 (for example, Lpin1 [probe set 98892_at]), only Id2 was upregulated in rat retina, brain, and spinal cord after injury (Table 3.2). In injured rat retina, $I d 2$ also displayed a chronic upregulation even after 30 days postinjury (Table 3.2; [GEO data set GSE1001, probe set rc_AI230256_at]). Id2 was also highly expressed during mouse retinal development at the time of high levels of cell proliferation and remained present in adult mouse retina [Retina Developmental Gene Expression, 
Figure 3.5. Evaluating candidate genes within chromosome 12 locus. A: The transcript variability of Id2, Crem, Egrl, Fos, Nr4al, Junb, Ptpn16 and Icaml in brains from BXD RI mouse strains is genetically linked to the regulatory locus on chromosome 12 . This network was generated by comparing genome-wide scans that measure the linkage of transcript variability across the mouse genome, as described in Figure 3.1. These transcripts are also upregulated as acute phase transcripts in retina, brain and spinal cord (Table 3.2). Transcripts genetically linked to the same regulatory loci and highly regulated in injured CNS are hypothesized to be part of a genetic network. B: The combined genome-wide map shows that their transcript expression has a strong genetic linkage to a locus within chromosome 12,10 to $30 \mathrm{Mb}$. The genome wide scan, zoomed at chromosome 12, shows the genetic linkage (y-axis, likelihood ratio statistic [LRS]) across the chromosome (x-axis, Megabase pairs [Mb]). This locus includes over 60 positional genes, some of which have a high or low single nucleotide polymorphism (SNP) density. SNP density is denoted by the height of the orange lines on the $\mathrm{x}$-axis. Several rules may help in selecting candidate gene responsible for this regulatory locus. The first rule is there have to be SNPs present within the coding or regulatory region of the gene of interest. As a first approximation we used SNP density that is displayed at the bottom of panel $\mathbf{B}$ and $\mathbf{D}$. The second rule is a high degree of transcript abundance variability in the BXD RI strains (illustrated in C). C: Transcript abundance variability in normal forebrains of BXD RI mouse strains is due to genetic polymorphisms between the parental C57BL/6 and DBA/2J mouse strains. The graph illustrates transcript abundance variability ( $\mathrm{y}$-axis) for genes (dots) within the 10 to $30 \mathrm{Mb}$ interval of chromosome 12 (xaxis). For a transcript to be mapped its abundance must vary across the BXD RI mouse strains. The higher the variation in transcript abundance the more likely that the gene is a candidate. Furthermore we expect that the variability is due to a polymorphism in the candidate gene, suggesting that the polymorphic gene is modulating its own expression level (that is, a "cisregulatory locus"). We measured transcript variability using an analysis of variance (ANOVA) that tests the between-strain variance compared with the total variance for 100 arrays from 35 mouse strains. The degrees of freedom for the between-group and total variance are 34 and 99. Strain-specific variation is significant $(\mathrm{p}<0.05)$ when $\mathrm{F}_{34,99}>1.5$. In the graph, each dot represents a gene with its variability measured by the ANOVA F-statistic (y-axis) and its genomic location within chromosome 12 described in Mb. Lpin1 and Id2 are polymorphic genes that displayed significant transcript variability and cis-regulatory loci in normal forebrains of BXD mouse strains. D: The third criterion identifies the genes that display self regulation across CNS tissues. For example, the transcript variability of $I d 2$ (red circle in $\mathbf{C}$ ) displayed a cis-regulatory locus in the brain (LRS=44, probe set 93013_at) and cerebellum (LRS=14; 1453596_at_A) of BXD RI mouse strains. Here we illustrate linkage of Id2 transcript variability in BXD RI mouse strains across chromosome 12. Red triangle indicates gene location. Lpin1 also displayed cisregulatory loci in brain and cerebellum (data available at GeneNetwork). E: The structure of the Id2 gene illustrates a SNP at the promoter region and four SNPs within the second intron. F: The SNP within the promoter region (Ensembl SNPView ID rs4229289 and Celera SNP ID $\mathrm{mC22302957)}$ is located within a highly conserved region and is adjacent to a nuclear transcription factor Y (NF-Y) binding site (TRANSFAC ID M00185). Gene structure was obtained from Ensembl Genome Browser. The TRANSFAC 5.0 database was accessed through the MOTIF website. Highly conserved regions were defined using the Genome Browser Conservation tool [Mouse May 2004 Assembly]. 

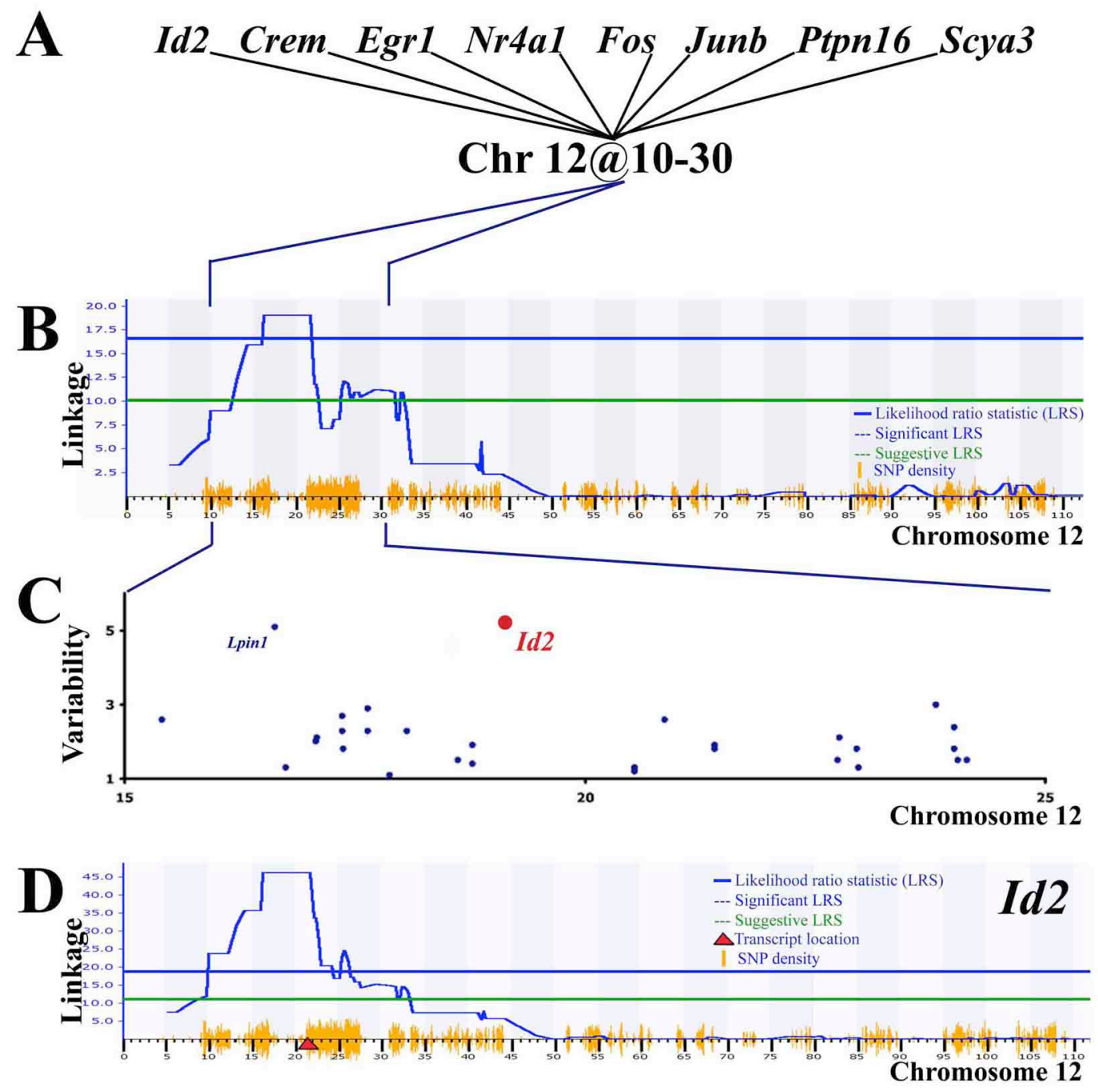

$\mathbf{E}$

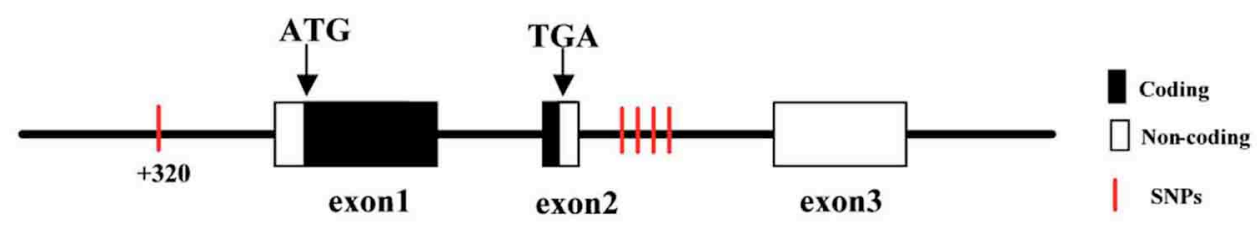

F

DBA2 GGTTCTTCCGCGTTCGCAGCCAATACC

B6 GGTTCTTCCGCGTTCGCAGCCAATGCC

Rn. GGTTCTTCCGCGTTCGCAGCCAATGCC

Hs. GGTTCTTCCGCGTTCGCAGCCAATCCC

NF-Y binding site GCCAATC

GCCAATG 
probe set rc_AI230256_at; and Mouse Retina SAGE Library, Mm.34871]. Third, we located one SNP for Id2 within the promoter and four SNPs within the second intron (Figure 3.5D; Ensembl gene ID ENSMUSG00000020644). An SNP within the promoter region of Id2 may alter the ability of a transcription factor to modulate $I d 2$ transcription.

We used in silico promoter analysis to define the transcription factor binding site affected by the SNP (Ensembl SNPView ID rs4229289 and Celera SNP ID mC22302957). Located 320 base pairs upstream of the starting codon, the SNP consisted of an adenine-guanine (A/G) substitution between the DBA/2J and C57BL/6. The SNP was also located in a highly conserved region across mouse, rat, and human orthologs (Figure 3.5F). Using the MOTIF database (www.motif.genome.jp), we determined that the SNP sits next to a CCAAT binding site (TRANSFAC ID M00254), a likely binding site for nuclear transcription factor (NF)-Y (TRANSFAC ID M00185). Based on the TRANSFAC 5.0 database available through the MOTIF website, NF-Y binds to the CCAAT motif when the adjacent 3'-nucleotide is either G or $\mathrm{C}$ as seen in the C57BL/6, rat, and human sequences for Id2 (Figure 3.5F). The nucleotide substitution of an A for a G/C may account for the lower Id2 levels seen in the DBA/2J and in all the BXD strains carrying the DBA/2J haplotype. Several lines of evidence indicate that Id2 is a good candidate gene for the regulatory locus on chromosome 12. Id2 is conserved and has a selfregulatory role in mouse brain and cerebellum. It is expressed in the developing retina during the critical time of cell proliferation and cell specification. It is upregulated immediately following injury to rat retina, brain, and spinal cord.

\section{Discussion}

Discovering mechanisms controlling retinal wound healing from gene expression data is a promising and challenging task. The method of extracting genetic networks and gene regulation presented here involves (1) highlighting robust injury genes in multiple models of CNS trauma, (2) 
defining regulatory loci using genetic analysis of transcript data (that is, expression genetics [Broman, 2005]) from CNS tissues, and (3) predicting candidate regulators using bioinformatic resources that are available online. Using this approach, we defined a group of acute phase genes that is commonly expressed in the retina, brain, and spinal cord after traumatic injury. Our expression genetic analyses revealed that regulatory loci on chromosomes 6,12 , and 14 control the expression of acute phase genes in brains from BXD RI mouse strains. With the help of an array of online bioinformatic tools, we identified three genetic networks and candidate upstream regulators: $N d r g 2, C c n d 2$, and $I d 2$.

Surprisingly, the changes controlled by these three loci are present in a variety of different CNS tissues: retina, brain, and spinal cord. This begs the question, are the networks controlled by the loci on chromosomes 6,12 and 14 unique to the CNS? If one examines normal tissues, these regulatory loci appear to be unique to the CNS. For example, expression genetics of hematopoietic stem cells from BXD RI mouse strains showed completely different sets of regulatory loci [Bystrykh et al., 2005]. This is not surprising given the difference in expression patterns between CNS tissues and hematopoietic stem cells [Chesler et al., 2005; Bystrykh et al., 2005]. The same is true of the liver where Schadt and colleagues [Schadt et al., 2003] identified a different set of regulatory loci that did not correlate with our observations in the CNS. Although the specificity of expression-related regulatory loci in CNS, hematopoietic stem cells, and liver are different in normal resting conditions, we cannot exclude the possibility that some regulatory mechanism are similar following trauma. Based upon our current data the loci at chromosomes 6,12 and 14 identified appear specific to the CNS.

Our method complements approaches that identify modules of co-regulated genes, shared cis-regulatory motifs, and transcriptional factors binding to cis-regulatory motifs [Livesey et al., 2000]. In reality, transcriptome changes during the wound-healing response are controlled by a much more complex network [Ridet et al., 1997; Geller et al., 2001; Bringmann and Reichenbach, 2001]. Our method holds the potential for identifying members of genetic networks and regulators that may include gene products involved in mRNA stability, heterochromatin remod- 
eling, transcriptional repression, and other regulatory mechanisms [Chesler et al., 2005; Bystrykh et al., 2005; Schadt et al., 2003]. Indeed, even indirect modifiers of the transcriptional response to injury that are not transcription factors can be identified using a genetic approach. Our analysis suggests, for example, that the expression of acute phase genes is genetically linked to the transcript expression of three non-transcription factors including the hydrolase NDRG2, the cyclin CCND2, and the dominant negative helix-loop-helix protein ID2.

Key to defining modulators of regulatory networks in BXD RI mouse strains is the genetic differences between parental strains. Genetic differences between C57BL/6 and DBA/2J strains provide a powerful system to study regulators and their targets, but this approach is limited to the detection of polymorphisms between these strains. For some genes believed to be involved in the response of the CNS to injury there are no genetic differences between the BXD parental strains. For example, STAT3 (a potent activator of acute phase genes such as Fos [May et al., 2003; Yang et al., 2003]) has no SNPs in the coding or regulatory regions when the sequence of Stat3 of C57BL/6 strain is compared to that of the DBA/2J strain. Consequently, expression genetics of BXD RI mouse strains revealed no genetic linkage between the Stat3 locus and genes known to be associated with its regulation such as Fos. To provide a fuller examination of the networks controlling retinal wound healing the BXD RI strains can be supplemented by studying additional strains. Other RI strains of mice can be equally valuable. The BXA and AXB RI strains were produced from crosses of the C57BL/6 (B) and A/J (A) mouse strains. Genetic differences between these two strains can be used to map different regulatory loci. One prime example of the use of the AXB RI strains comes from a study of horizontal cell density. A two-fold difference in horizontal cell density was observed between C57BL/6 and A/J mouse strains [Williams et al., 1998]. The genetic backgrounds of the A/J mouse strains and RI strains derived from the $\mathrm{C} 57 \mathrm{BL} / 6$ and $\mathrm{A} / \mathrm{J}$ mouse strains make it possible to map genetic loci controlling horizontal cell number and potentially the response of horizontal cells to retinal injury. Undoubtedly as more genetically diverse sets of strains become available, transcriptome-wide analysis combined with genetic analysis will yield additional regulatory loci. This type of analysis and the 
development of additional expanded RI strains of mice hold great promise in the search for genetic networks controlling complex biological processes such as wound healing in the retina and CNS.

Typically, a candidate upstream modulator within regulatory loci will display (1) high transcript variation that maps onto its transcript location, that is, a cis-regulatory locus, (2) polymorphisms between parental strains, and (3) a function related to CNS disease or expression changes after CNS injury. $N d r g 2, C c n d 2$, and $I d 2$ met the candidate gene criteria for the regulatory loci on chromosomes 14,6 , and 12, respectively. N-myc downstream regulated gene 2 (NDRG2) is found at high protein levels in neurogenic regions of the adult rat brain. In these regions, NDRG2 localizes to GFAP-positive astrocytes or radial glia [Nichols, 2003]. In humans, down-regulation of $\mathrm{Ndrg} 2$ is associated with glioblastoma [Deng et al., 2003]. High levels of NDRG2 are often associated with senile plaques of Alzheimer's patients and cellular processes of dystrophic neurons [Mitchelmore et al., 2004]. Our meta-analyses of Ndrg2 using transcript data from developing mouse retina showed that $N d r g 2$ is expressed during the time of cell proliferation and cell specification. These data suggest that NDRG2 may play a key role in the proliferation of glia cells and the degeneration of neurons.

A second likely upstream candidate of CNS injury is cyclin D2 (CCND2). Cyclin D2 phosphorylates the $\mathrm{Rb}$ protein of the RB-E2F complex. Cyclin D2 binding to the Rb protein allows the transcription factor E2F to enter the nucleus and stimulate proliferation. During the development of the cerebellum, cyclin D2 is a marker of proliferating granule cell precursors. Granule neurons are the principal neuronal component of cerebellum, and mice deficient in cyclin D2 show decreases in the number of granule cells and stellate interneurons [Diaz et al., 2002; Huard et al., 1999]. Our meta-analyses of $C c n d 2$ also indicated that $C c n d 2$ is moderately expressed in cultured astrocytes from the cortex and optic nerve, in the mouse developing retina, and injured rat retina. The enhanced expression of cyclin D2 in the retina following trauma may contribute to reactive responses such as cellular proliferation. 
The third and most promising upstream candidate of CNS injury is the inhibitor of DNA binding 2 (ID2). Our analyses suggest that changes in Id2 expression are a common theme in many reactive CNS tissues. Based on our meta-analyses of microarray data, $I d 2$ is upregulated in rat retina, brain, and spinal cord after mechanical injury. In rat models of glaucoma [Ahmed et al., 2004], the dystrophic retina also upregulates Id2 [personal communication, Farid Ahmed and Stanislav Tomarev, NEI, NIH]. We have confirmed upregulation of $I d 2$ transcript in mouse retina after a mechanical and toxic injury [Vazquez-Chona personal observation]. Optic nerve head astrocytes from patients suffering glaucoma display higher Id2 transcript levels than astrocytes from non-glaucomatous patients [Yokota and Mori, 2002]. The role of ID2 as a positive regulator of cell-cycle progression [Yokota and Mori, 2002; Toma et al., 2000] is consistent with the proliferative response that occurs in retinal injury. A potential source for dividing cells in the inner nuclear layer is the Muller cells [Dyer and Cepko, 2000; Fischer and Reh, 2003]. When Muller cells enter the cell cycle, they decrease the expression of cell cycle inhibitors, including the cyclin-dependent kinase inhibitor p27/Kip1 [Dyer and Cepko, 2000]. ID2 is known to negatively regulate the transcription of cell cycle inhibitor genes, including p15, p16, and p21 [Yokota and Mori, 2002]. ID2 downregulates expression of cell cycle inhibitors by preventing the interaction of basic helix-loop-helix (bHLH) transcription factors that promote the transcription of cell cycle inhibitor genes [Yokota and Mori, 2002]. Alternatively, ID2 may stimulate proliferation by binding to the unphosphorylated $\mathrm{Rb}$ protein, allowing the release of the transcription factor E2F. E2F in turn activates genes involved in G1-S transition and hence proliferation [Yokota and Mori, 2002]. Re-entry into the cell cycle may also indicate apoptotic activity [Klein and Ackerman, 2003]. ID2 is known to play a role in neuronal apoptosis [Gleichmann et al., 2002]. Cerebellar granule neurons upregulate transcript and protein levels of ID2 during the onset of apoptosis [Gleichmann et al., 2002]. The expression of Id2 antisense RNA in neurons protects from apoptosis. Together these data suggest that the increase in ID2 may stimulate cells of the INL into the G1-S transition of the cell cycle either for proliferation or apoptosis. We are currently using the genetic background offered by the BXD RI mouse strains to determine the role of the 
chromosome 12 locus and ID2 in retinal wound healing. We have confirmed the variability of ID2 at the transcript and protein level in retinas of BXD RI mouse strains [Vazquez-Chona personal observation]. We are using high and low Id2 expressers to see the effect of $I d 2$ dose effect on wound healing events following retinal trauma.

The novel combination of microarray analysis, expression genetics, and bioinformatics provides a new and powerful approach to defining regulatory elements in the genome. Using this approach, we were able to generate specific, testable hypotheses defining pathways that regulate proliferative and reactive responses in the retina and elsewhere in the CNS. As more diverse gene expression data sets become available, it is our belief that comparison of gene expression and regulation in different biological contexts will help identify the regulatory elements controlling the reactive response in the retina. 


\title{
Chapter 4
}

\section{Genetic Influences on Gene Expression and}

\author{
Retinal Wound Healing
}




\section{Introduction}

Discovering the mechanisms that control retinal wound healing from large-scale analysis of gene expression is a promising and challenging task. Gene expression profiling of retinal wound healing revealed that changes in the transcriptome have specific spatial and temporal patterns [Vazquez et al., 2004; Chen et al., 2004; Yoshimura et al., 204; Wilson et al., 2003; Ahmed, et al., 2004; Gerhardinger et al., 2005; Rattner and Nathans, 2005]. The retinal response to trauma involves changes that start at the stress site and then spread to include the entire retina [Vazquez et al., 2004]. Transcriptome-wide changes are highly regulated into three temporal patterns of expression-early acute, delayed subacute, and late chronic phases. Genes within each phase are functionally related and reflect the known cellular changes. Transcriptome profiling of injured retina also revealed that global changes are highly similar across different injury models, including mechanical trauma, ischemia, and increased intraocular pressure [Vazquez et al., 2004; Yoshimura et al., 204; Ahmed, et al., 2004]. Together the growing collection of retinal transcriptome profiles is cataloging the genes that underlie the biochemical and cellular changes of wound healing. The next level of analyses for gene expression data involves defining the networks and regulators that control specific wound healing processes such as cell death and tissue remodeling.

Our approach to defining regulatory mechanisms from transcriptome data involves (1) highlighting networks of genes sharing similar patterns of expression across injury models and (2) defining the location of gene expression modulators using genetic analyses of genes expression (that is, expression genetics). Comparative analysis of gene expression and gene regulation across different biological contexts and different tissues can help define key networks, as well as, define tissue specific networks [Segal et al., 2005]. Using this approach, we previously defined a group of acute phase transcripts that is commonly upregulated in injured retina, brain, spinal cord. In this group we found transcription factors and transcription repressors as well as cell activating factors such as chemokines [Vazquez et al., 2005]. In contemporary transcriptome research, observations of highly co-expressed genes are supplemented by higher-level analyses 
aiming at discovering the networks controlling gene expression. One approach combines transcriptome profiling with genetic linkage analysis to reveal the location of the genetic determinants that modulate gene expression variability. Expression genetics has been instrumental in identifying genetic determinants and gene expression phenotypes underlying complex traits, including disease and behavior [Aitman et al., 1999; Karp et al., 2000; Berge et al., 2000]. In an example of gene expression genetics, Chesler and colleagues [2005] identified genome-wide loci that modulate gene expression phenotypes in forebrains of a genetically variable population, the $\mathrm{BXD}$ recombinant inbred $(\mathrm{RI})$ mouse strains. BXD RI strains were derived from experimental crosses between the D57BL/6 and DBA/2J strains. Using their database available at GeneNetwork [www.genenetwork.org], we made the surprising finding that a group of genes upregulated in the retina, brain, and spinal cord share co-regulation of their expression levels in forebrains of BXD RI mouse strains. Expression of acute phase genes in BXD RI mouse forebrains was modulated by genetic differences within the three loci on chromosomes 6, 12, and 14 [Vazquez et al., 2005]. This finding raised the hypothesis that these loci modulate the acute phase response of the CNS.

In this study, we specifically test the hypothesis that loci on chromosomes 6,12 , and 14 modulate retinal wound healing. We determine the specificity of the loci by comparing regulatory loci across functional groups and tissues. We define candidate genes using an integrated bioinformatic approach that combines online databases, defining single nucleotide polymorphisms and gene expression profiling in the injured and developing CNS. We test the hypothesis by examining the direction of flow and targets in wound healing models where predicted modulators are expressed at different expression levels. This approach illustrates an integrated bioinformatic approach that generates specific, testable hypothesis from gene expression data. 


\section{Methods}

\section{Meta-analyses of Microarray Data Sets}

This study was designed to define networks and regulators from our gene expression data of injured rat retina [Vazquez et al., 2004]. The data set is available online at Gene Expression Omnibus (GEO, www.ncbi.hlm.nih.gov/geo) as the group series GSE1001. This data set surveyed the temporal gene expression profiles of rat retinal wound healing ( $4 \mathrm{~h}$ and $1,3,7$, and 30 days) after a mechanical tear. Our working group of wound-healing genes was defined by comparing injured retinal time points versus normal retina and using three criteria: fold changes $>| \pm 2|$, Student's ttest $(\mathrm{P}<0.05)$, and genes with moderate-to-high expression levels (please see Chapter 2 for discussion on expression thresholds). To complement our analysis of wound-healing genes, we obtained publicly available microarray analyses that examined the transcriptome response of developing mouse retina [Dorell et al., 2004]; the transcriptome of cultured mouse neurons [Kraft et al., 2004], mouse astrocytes [Kraft et al., 2004] rat Muller cells [Gerhardinger et al., 2005], human optic nerve astrocytes [Yang et al., 2004], human U87 glioma cells [unpublished data, Eldon Geisert], and rat RPE cells [unpublished data, Eldon Geisert]; and the genetics of gene expression in forebrains from 35 BXD RI mouse strains (available at GeneNetwork, [Chesler et al., 2005]). We made clear in Chapter 3 (Table 3.1) the details and availability of each microarray data set. For these analysis, we obtained the raw data (CEL files), determined signal values using Microarray Suite 5.0 (MAS 5.0; Affymetrix, Santa Clara, CA), transformed signals to a log scale (base 2), and normalized microarray mean intensity to 8 as described previously [Vazquez et al., 2004]. These transformations yielded signal intensities ranging from 1 to 18 relative units of fluorescence. Further analyses included only transcripts with medium to high abundance (that is, signals greater than 8.64 [Vazquez et al., 2004]).

The use of microarray data sets involved three different meta-analyses. First, we used GeneNetwork to define regulatory loci shared by wound-healing genes (details below). Second, we examined temporal expression patterns of wound-healing genes in the developing mouse ret- 
ina [Dorell et al., 2004] and in the injured rat retina [Vazquez et al., 2004]. Third, we determined the expression level of wound-healing genes in cultured astrocytes, cultured Muller cells, cultured microglia, and cultured neurons. A wound-healing gene was considered expressed in a tissue if it displayed medium to high abundance. Comparison of rat genes across species was assayed by finding the corresponding ortholog's probe set(s) using Affymetrix NetAffex (June 2005 Annotation) and Ensembl AffyProbe (v33) database. Since gene names may differ across species and multiple probe sets may be available, we include the probe set identifier when necessary.

\section{Online Regulatory Locus Analyses}

Regulatory loci modulating gene expression in forebrains of BXD RI mouse strains were defined using GeneNetwork [www.genenetwork.org]. Details on the methods, data, and analyses are available at GeneNetwork [Chesler et al., 2005; Bystrykh et al., 2005]. We described the BXD RI mouse strains and GeneNetwork in Chapter 3. Briefly, GeneNetwork is a suite of databases and analysis software that identifies genomic loci that control transcriptome differences in brain regions in a panel of $32 \mathrm{BXD}$ RI mouse strains derived from C57BL/6 (B) and DBA/2J (D) mice, both parental strains, and the F1 hybrid (a total of 35 isogenic lines [Chesler et al., 2005; Bystrykh et al., 2005]). Using quantitative genetic analysis and a genetic map consisting of 779 fully genotyped markers, GeneNetwork correlates expression variability to genotypes at locations throughout the mouse genome. The average distance between adjacent markers is approximately 4 megabases $(\mathrm{Mb})$. Variability across strains was measured using analysis of variance (ANOVA) testing the between-strain variance compared with the total variance for 100 arrays from 35 mouse strains. The degrees of freedom for the between-group and total variance are 34 and 99 . Strain-specific variation is significant $(\mathrm{p}<0.05)$ when $\mathrm{F}_{34,99}>1.5$. We used the published, expression genetics data of mouse forebrain [UTHSC Brain mRNA U74Av2 HWT1PM, December 2003] and of mouse hematopoietic stem cells [GNF Hematopoietic Cells U74Av2 RMA, March 2004] to identify common and unique regulatory loci for wound-healing genes. We also supple- 
ment the forebrain data with unpublished expression genetics data of mouse striatum (HBP/Rosen Striatum M430V2 RMA. April 2005) and mouse cerebellum [SJUT Cerebellum mRNA M430 RMA, March 2005]. We also relate gene expression phenotypes to mouse neurological phenotypes using the BXD Published Phenotypes Database also available at GeneNetwork [database queried on November 5, 2005)]

\section{Online Bioinformatic Resources}

We used online databases and computational tools to identify candidate genes within a regulatory locus, to determine functional themes within a network of genes, and to query literature abstract databases for biological interactions. To identify candidate genes within a regulatory locus, we located genes within the appropriate genomic interval using the Genome Browser [http://genome.ucsc.edu, Mouse May 2004 assembly]. Single nucleotide polymorphisms (SNPs) were identified by comparing mouse strain genome sequences in the Celera SNP [Celera Discovery Systems, CA; July 2003 Assembly], Ensembl Mouse SNPView [v33], and Entrez SNP databases. Biological significance of a SNP was determined if the SNP affected a biological motif (for example, transcription factor binding sites and protein-protein interaction sites) using the MOTIF database [http://motif.genome.jp/]. Several online databases provide gene expression data or expression distribution in the developing and injured CNS. Gene Expression Omnibus (GEO) is a high-throughput gene expression abundance data repository, as well as an online resource for gene ex-pression data browsing, query, and retrieval [www.ncbi.nlm.nih.gov/geo]. Retina Developmental Gene Expression describes the gene expression profile of thousands of genes in the developing postnatal C57BL/6 mouse retina that were analyzed by hybridization to Affymetrix Mu74 Av2 [www.scripps.edu/cb/friedlander/gene_expression; Dorrell et al., 2004]. Mouse Retina SAGE Library provides serial analysis of gene expression tags or in situ hybridization images for a limited number of transcripts expressed in the developing and adult mouse retina [https://bricweb.partners.org/cepko/default.asp; Blackshaw et al., 2004]. Gene Expression Nervous System Atlas (GENSAT) maps the transcript distribution in the mouse CNS using in 
situ hybridiza-tion [www.ncbi.nlm.nih.gov/gensat]. To determine functional themes within a network of genes, we retrieved the Gene Ontology annotation data using WebGestalt (WEBbased GEne SeT AnaLysis Toolkit; [http://genereg.ornl.gov/webgestalt]). To mine literature abstracts for biological interactions such activation and inhibition, and directionality, we used an online tool Chilibot [http://www.chilibot.net, Chen and Sharp, 2004].

\section{Animals, Anesthesia, and Surgery}

Four strains of mice were used for this study $(n=48$ mice). These included the DBA/2J strain $(n$ $=20)$, BXD38 strain $(n=20)$, C57BL/6 strain $(n=4)$, and F1 progeny from a DBA/2J and C57BL/6 cross $(n=4)$. Strains were purchased from The Jackson Laboratory (Bar Harbor, ME) and colonies were maintained. The BXD38 strain is an inbred line derived from brother-sister matings starting from an F2 intercross [Taylor et al., 1999]. For all experiments we used adult, male mice (10 weeks old, 22-25 g). All protocols used in this study were approved by the Animal Care and Use Committee of the University of Tennessee Health Science Center and were in accordance with the Institute for Laboratory Animal Research and with the ARVO Statement for the Use of Animals in Ophthalmic and Vision Research. Prior to any retinal injuries, mice were deeply anesthetized with avertin (1.25\% 2,2,2-tribromoethanol and $0.8 \%$ tert-pentyl alcohol in water; 0.8-1.0 ml, intraperitoneal injection). Injured animals and non-injured animals used for gene and protein expression were deeply anesthetized with $\mathrm{CO}_{2}$. Animals used for immunohistochemistry were deeply anesthetized with avertin (1.0-1.2 ml, intraperitoneal injection) prior to perfusion. Retinal wound healing was induced via a mechanical tear, toxic injury, and optic nerve crushes. A tear was induced by penetrating the pars plana with a 27 -gauge needle and scraping the superior nasal retina, as previously described [Vazquez et al., 2004]. A toxic injury was induced by injecting kanaic acid into the vitreous ( $2 \mathrm{ng}$ diluted in sterilized saline). An optic nerve crush was performed using a binocular operating microscope, incising the conjunctiva of eye, exposing the optic nerve, and pressing on the nerve with a cross-action forceps for 15 seconds. 


\section{Cell Culture Studies}

Mouse astrocytic C8-D1A cells (ATCC, American Tissue Culture collection; CRL-2541) were routinely maintained in Basal Medium Eagle (GIBCO, Carlsbad, California) supplemented with 5\% Fetal Bovine Serum (Intergen, Logan, Utah), D-Glucose (Sigma-Aldrich, St Louis, MO), Penstrep Antibiotic-Antimycotic (GIBCO), Gentamicin Sulfate (Sigma-Aldrich), and Sodium Bicarbonate (Sigma-Aldrich). Cells were maintained in an incubator at $37^{\circ} \mathrm{C}$ in $5 \% \mathrm{CO}_{2}$. Wound healing was modeled in culture by growing cell monolayers on 24-well plates and then scratching the monolayer with a sterile plastic $10-\square$ l pipette (Fisher Scientific, Hampton, NH). Morphologic response of astrocytes along the margin of the wound was followed by taking phase contrast micrographs at different time intervals following the wound. The rate of migration was measured by the distance that cells migrate into the wound at different time points. Cell cycle dynamics were analyzed with flow cytometry. Cells in single cell suspension were fixed with ice cold $70 \%$ ethanol. After the ethanol was washed off, cells were treated with $1 \%$ BSA in PBS to block nonspecific binding. To stain DNA, cells were incubated with propidium iodine $(10 \mathrm{ug} / \mathrm{mL}$; SigmaAldrich) and RNAse A (1 mg/mL; Sigma-Aldrich) in PBS at $37^{\circ} \mathrm{C}$ for $30 \mathrm{~min}$. Samples were then analyzed with a cytometer (Becton Dickinson FACSCalibur, BD Biosciences). Cell viability was assayed with $0.4 \%$ trypan blue and examined by low-power microscopy.

\section{RNA Interference}

Using conventional criteria, we designed 21-base short interfering RNAs (siRNAs) that (1) avoid the 75 bases downstream of the start codon, (2) form a 19-base core duplex with 3'TT overhangs, (3) contain approximately 50\% G/C content, and (4) display target specificity. siRNAs targeted three sites of the Id2 mRNA (NM_010496, at 568, 898, and 1131; Table 4.1). Sense and antisense strands were annealed as recommended by manufacturer (Integrated DNA Technologies, Coralville, IA). Control siRNA sequences included a scrambled siRNA duplex and a Cy3-labeled scrambled siRNA duplex (Dharmacon, Lafayette, CO). siRNA duplexes were transfected using Oligofectamine (Invitrogen, Carlsbad, CA) as recommended by the manufacturer. Briefly, $2 \times 10^{5}$ 
Table 4.1. Oligonucleotides used for RNA interference and real-time RT-PCR.

\begin{tabular}{|c|c|c|}
\hline Targets & Accession No. & Sequence $(5 \mathbb{W}$ 3ם \\
\hline Id2_siRNA_l & NM_010496 & AUUCAACGUGUUCUCCUGGdTdT \\
\hline$I d 2 \_s i R N A \_2$ & NM_010496 & $\begin{array}{l}\text { CCAGGAGAACACGUUGAAUdTdT } \\
\text { ACAGCAUUCAGUAGGCUCGdTdT }\end{array}$ \\
\hline Id2_siRNA_3 & NM_010496 & $\begin{array}{l}\text { CAAAGCACUGGUUGUCUGAdTdT } \\
\text { UCAGACAACCAGUGCUUUGdTdT }\end{array}$ \\
\hline $\operatorname{Id} 2$ & NM_010496 & $\begin{array}{l}\text { GTCCTTGCAGGCATCTGAAT } \\
\text { CTTAGTTTTCCTTCCGCTTTCTT }\end{array}$ \\
\hline Lpin1 & NM_015763 & $\begin{array}{l}\text { CCCCATTCCTCATAGCTCAA } \\
\text { CACTAGTGGCTCCTCCTTGC }\end{array}$ \\
\hline Fos & NM_010234 & $\begin{array}{l}\text { AGAATCCGAAGGGAACGGAA } \\
\text { GGTCGTTGAGAAGGGGCAG }\end{array}$ \\
\hline Stat3 & NM_21365 & $\begin{array}{l}\text { TGTTGGAGCAGCATCTTCAG } \\
\text { CTTGGCTCTTGAGGGTTTTG }\end{array}$ \\
\hline Casp3 & NM_00981 & $\begin{array}{l}\text { CCTCAGAGAGACATTCATGGC } \\
\text { TCGGCTTTCCAGTCAGACTC }\end{array}$ \\
\hline Crygd & NM_007776 & $\begin{array}{l}\text { AGCAGTGGATGGGTTTCAG } \\
\text { GTGGAATCGGTCCTGGAG }\end{array}$ \\
\hline$C d 81$ & NM_133655 & $\begin{array}{l}\text { CTGTTTGCCTGTGAGGTGG } \\
\text { TCAGTGTGGTCAGTGCGTT }\end{array}$ \\
\hline Gfap & NM_010277 & $\begin{array}{l}\text { AGGGACAATCTCACACAGGAC } \\
\text { CTCCAGCGACTCAACCTTC }\end{array}$ \\
\hline Gapdh & NM_001001303 & $\begin{array}{l}\text { TCCCACTCTTCCACCTTCGATG } \\
\text { GTCCACCACCCTGTTGCTGTA }\end{array}$ \\
\hline Rps18 & XM_215328 & $\begin{array}{l}\text { CTCGCTCCTCTCCTACTTGG } \\
\text { ACCGGGTTGGTTTTGATCT }\end{array}$ \\
\hline
\end{tabular}


cells and $1 \times 10^{5}$ cells were seeded in 12 -well and 24-well plates. Transfection was optimized using $50 \%$ confluent cells and $50 \mathrm{nM}$ siRNA duplex concentration. For functional studies, we used a second siRNA transfection $24 \mathrm{hrs}$ after the first transfection.

\section{Real Time RT-PCR}

Total RNA from was isolated and treated with DNase using the SV Total RNA Isolation kit (Promega, Madison, WI). Transcript levels were measured using quantitative real-time reverse transcription-polymerase chain reaction (qRT-PCR). We performed primer design, qRT-PCR reaction, and RT-PCR analysis as described in Chapter 2. Nucleotide sequences are shown in Table 4.1. We performed qRT-PCR reactions in the iCycler (BioRad, Richmond, CA) using the reagents in the SYBR Green RT-PCR kit (Applied BioSystems, Warrington, UK). To determine the relative change in gene expression, we compared the number of cycles $(\mathrm{Ct})$ needed to reach the midpoint of the linear phase using the iCycler analysis software. All observations were normalized either to the housekeeping genes Gapdh or Rps18.

\section{Protein Expression and Localization}

To quantify expression levels, we used standard immunoblot methods [Vazquez and Geisert, 2000]. Proteins were extracted from cells and tissue by homogenization in $2 \%$ Sodium Dodecyl

Sulfate (SDS) in 50nM TRIS buffer $(\mathrm{pH}=7.5)$. Equal quantities of proteins were separated on 4$15 \%$ SDS-polyacrylamide gel electrophoresis (SDS-PAGE) and transferred to nitrocellulose membranes. Primary antibodies included hamster anti-CD81 (Eat2; Becton Dichinson, San Jose, CA), rabbit anti-GFAP (Company), rabbit-anti ID2 (Santa Cruz Biotechnology, Santa Cruz, CA), rabbit anti-ezrin (Santa Cruz Biotechnology), rabbit anti-STAT3 (Santa Cruz Biotechnology), and rabbit anti-PCNA (Santa Cruz Biotechnology). Primary antibodies were detected with peroxidase-labeled secondary anti-rabbit, anti-mouse, and anti-hamster antibodies (Jackson ImmunoResearch, West Grove, PA). 
Indirect immunohistochemical methods were used to define the cellular localization of GFAP [Vazquez and Geisert, 2000]. For the purpose of immunohistochemistry, deeply anesthetized animals were perfused through the heart with saline and $4 \%$ paraformaldehyde in $0.1 \mathrm{M}$ phosphate buffer. Eyes were removed and fixed in $4 \%$ paraformaldehyde, stored in $30 \%$ sucrose, and sectioned to obtain $16-\mu \mathrm{m}$ frozen sections. To retrieve cross-linked cytoplasmic antigens, we treated retinal sections with either a solution of $1 \mathrm{mmol} / \mathrm{L}$ ethylene glycol-bis (2aminoethylether)-N,N,N',N'- tetraacetic acid (EGTA, pH 8.4; Sigma-Aldrich, St. Louis, MO) and $0.3 \mathrm{M}$ sucrose for $20 \mathrm{~min}$ at $80^{\circ} \mathrm{C}$. For immunohistochemistry of cells, cells were grown and transfected on 8-well Poly-D-Lysine Coated Culture Slide (BD Biosciences, Bedford, MA). Cells were fixed with $4 \%$ paraformaldehyde and permeabilized with $5 \%$ acetic acid in ethanol. Sections and cells were washed in borate-buffered saline (BBS, pH 8.0; Sigma-Aldrich) and blocked in $4 \%$ bovine serum albumin (BSA; Sigma-Aldrich) and 0.1\% Triton X-100 (Sigma-Aldrich). Rabbit polyclonal anti-GFAP (1:100 dilutions; Company) and FITC donkey anti-rabbit IgG (1:200 dilutions; Jackson ImmunoResearch, West Grove, CA) were dissolved in BBS, 0.4\% BSA, and 0.1\% Triton X-100. DNA was stained with 4,6-diamino -2-phenylindole (DAPI; Sigma-Aldrich), and TOPRO-3 (Invitrogen). For fluorescence microscopy, sections were examined with a confocal laser-scanning microscope (MRC-1024; Bio-Rad, Hercules, CA). No immunoreactivity was observed in normal or injured retina when primary antibodies were omitted.

\section{Results}

\section{Specificity and Targets of Chromosome 12 Locus}

We examined regulation genes occurring during the process of CNS wound-healing (acute, subacute, and chronic phase genes) using gene expression genetics of forebrains in 35 BXD RI strains. Our meta-analyses revealed that a group of wound-healing genes (Appendix C, Table C.1) shared genetic linkages to loci on chromosomes 6, 12, and 14 (Figure 4.1A). The linkage 
Figure 4.1. Chromosome 12 locus modulates wound-healing mechanisms in the CNS. Genetic linkage maps show the regulation of gene expression in BXD recombinant inbred (RI) strains. This regulation is based on the genetic correlation of gene expression (individual rows on the $y$ axis) to genomic markers across the mouse genome (x-axis) as described in Chapter 3. Blue hues represent correlations for elevated gene expression in mice with C57BL/6 allele at a given locus, and orange hues represent correlation for elevated gene expression in mice with DBA/2J allele.

A: Expression of wound healing genes in mouse forebrains is controlled by three regulatory loci on chromosomes 6, 12, and 14. B: Synaptic-related genes in mouse forebrains are also controlled by regulatory loci on chromosomes 6 and 14. C: Wound-healing genes shared no regulatory loci in hematopoietic stem cells. D: Published data from phenotypes in BXD RI strains further support that chromosome 12 locus also associates with neurological phenotypes. The C57BL/6 allele for the locus is one of the loci associated with resistance to noised-induced cell death of spiral ganglion cells, the cells innervating auditory receptors cells [GeneNetwork ID 10652 and 10655; Willott and Erway, 1998]. The DBA/2J allele of this locus also predisposes susceptibility to generalized convulsions when exposed to intense auditory stimulation [GeneNetwork ID 10434-5, 10437-8, and 10504; Neumann and Collins, 1991]. In contrast, the C57BL/6 allele is one of the loci that confers enhanced neurogenesis and survival of new neurons and astrocytes in adult hippocampus [GeneNetwork ID 10338, 10340-1, and 10378; Kempermann and Gage, 2002]. Linkage maps were generated using the Interval Mapping and Cluster Tree tools at GeneNetwork [UTHSC Brain mRNA U74Av2 HWT1PM, December 2003; GNF Hematopoietic Cells U74Av2 RMA, March 2004; HBP/Rosen Striatum M430V2 RMA, April 2005; SJUT Cerebellum mRNA M430 RMA, March 2005; BXD Published Phenotypes, queried November 2005]. 

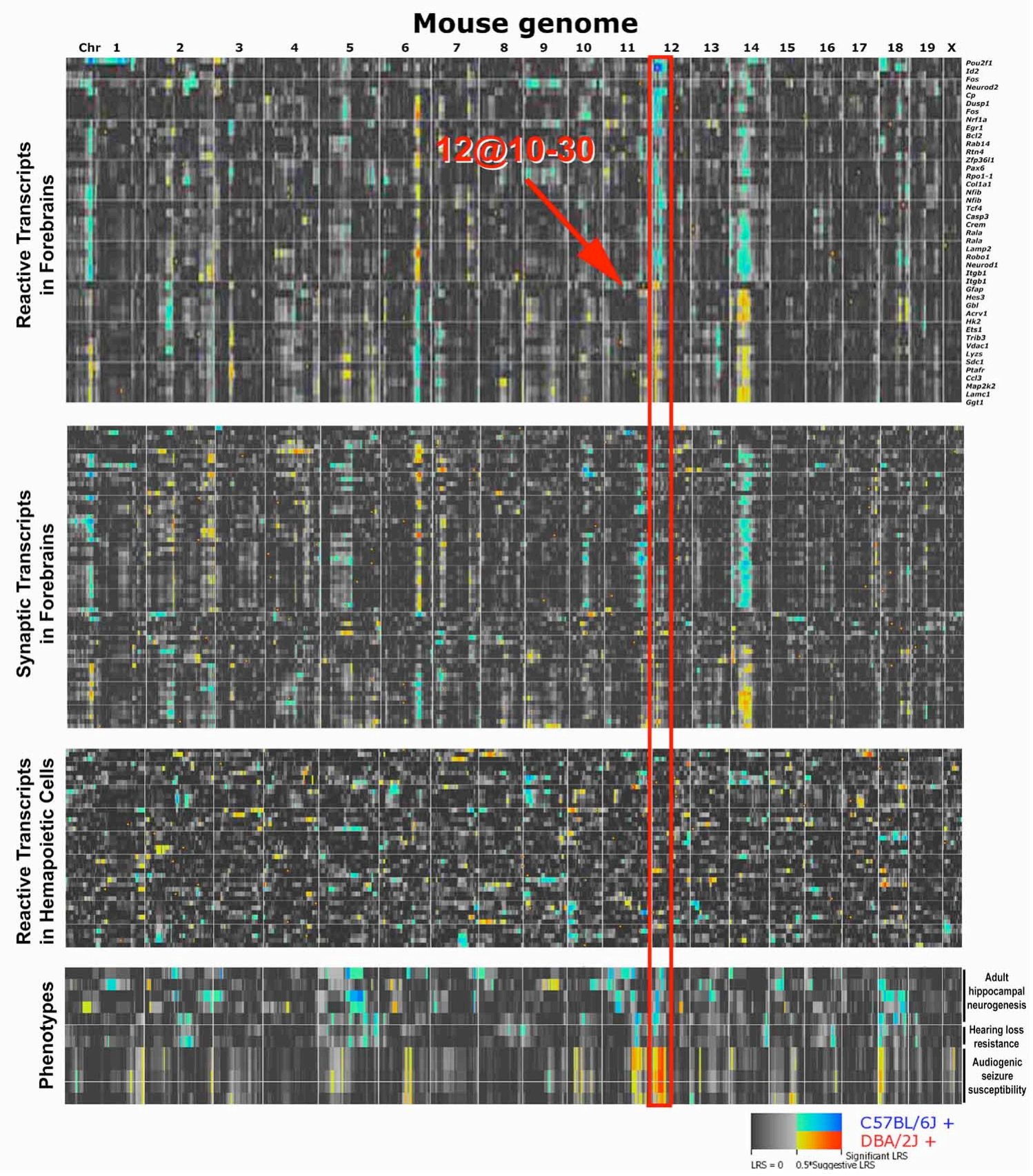
mapping of these genes suggest that gene expression is a complex trait with multiple loci affecting gene expression phenotype. To define the specificity of regulatory loci, we compared the loci controlling the expression of wound-healing genes to the loci controlling synaptic-related genes. Wound-healing and synaptic-related genes shared regulatory loci on chromosomes 6 and 14 (Figure 4.1A and 4.1B). The only locus unique to wound-healing genes was chromosome 12 locus. The uniqueness of this locus was further tested by comparing the loci controlling woundhealing genes in mouse forebrains and in hematopoietic stem cells. Wound-healing genes shared no regulatory loci in hematopoietic stem cells (Figure 4.1C). We also observed that chromosome 12 locus modulates three neurological phenotypes in BXD RI strains: audiogenic seizures, hearing loss based on spiral ganglion cell number, and neurogenesis of the adult hippocampus (Figure 4.1D). The comparison of regulatory loci across functional groups and across tissues reveals that the chromosome 12 locus specifically regulates the transcript expression of reactive transcripts in mouse forebrains.

The majority of the transcripts modulated by the chromosome 12 locus have their gene location outside this locus. Regulation of gene expression by an element outside the gene location is known as trans-regulation. Some of these trans-regulated genes are associated with the CNS response to injury: Fos, Nr4al, and Gfap. Regulation of gene expression by a genomic element within or adjacent to the gene location is termed cis-regulation. For example Id2 and Lpin1 lie within the locus (Figure 4.1A). Despite their diverse genomic locations, the expression of these genes is controlled by the segregating pattern of the C57BL/6 and DBA/2J alleles corresponding to chromosome 12, 10 to $30 \mathrm{Mb}$ (Figure 4.1A). On average when the C57BL/6 alleles are present, Fos, Nr4al, and Id2 are expressed at higher levels than when the DBA/2J alleles are present. The converse is true for Gfap: when the DBA/2J alleles are present Gfap is expressed at higher levels than when the C57BL/6 alleles are present. Since the pattern of expression of these transcripts in forebrains is correlated to the chromosome 12 locus, it follows that their expression patterns are correlated to each other. Indeed transcripts positively correlated to the same parental allele are positively correlated. For example, $\mathrm{Fos}, \mathrm{Nr} 4 \mathrm{al}$, and $\mathrm{Id} 2$ are positively co-regulated by 
C57BL/6 alleles and are also positively correlated with each other, whereas they are negatively correlated with Gfap, a transcript positively correlated to the DBA/2J allele (Appendix C, Table C.2). Gene expression associations such as co-regulation by the same genomic loci and significant expression correlation define genetic networks [Chesler et al., 2005]. Therefore, the woundhealing genes that are linked to chromosome 12 locus are part of a genetic network that controls their expression due to genetic differences between the parental strains (Figure 4.2A). Since these transcripts are also differentially expressed in injured retina, this network may also control wound-healing events in the retina and elsewhere in the CNS.

\section{Biological Processes Controlled by Chromosome 12 Network}

The most obvious approach to understanding the functional role of a genetic network is to examine the functions of gene products associated with the network. For the chromosome 12 network four functional themes emerged: regulation of transcription, cell death, cell proliferation, and neural development and differentiation (Figure 4.2B). A non-biased, statistical approach to defining the function of the network ( $\mathrm{n}=41$ genes) is to compare the observed number of regulated genes as compared to the expected number in a population belonging to a particular functional category. For the network we observed 27\% (11 out of 41 genes) of genes to be related to the regulation of neural development and differentiation. This percentage is higher than the percentage (7\%) observed among the total population of retinal reactive transcripts, and much higher than the percentage of expected genes in the entire genome. These bioinformatic-based results document that genes in chromosome 12 network participate in the regulation of transcription, cell death, cell proliferation, and neural development and differentiation. The functional significance of the network is further validated by defining known molecular associations among these gene products in each of these molecular processes. We queried the biological literature using textmining tools to illustrate networks of known biological interactions. Within the transcripts grouped into the neural development and differentiation category, the literature documents 

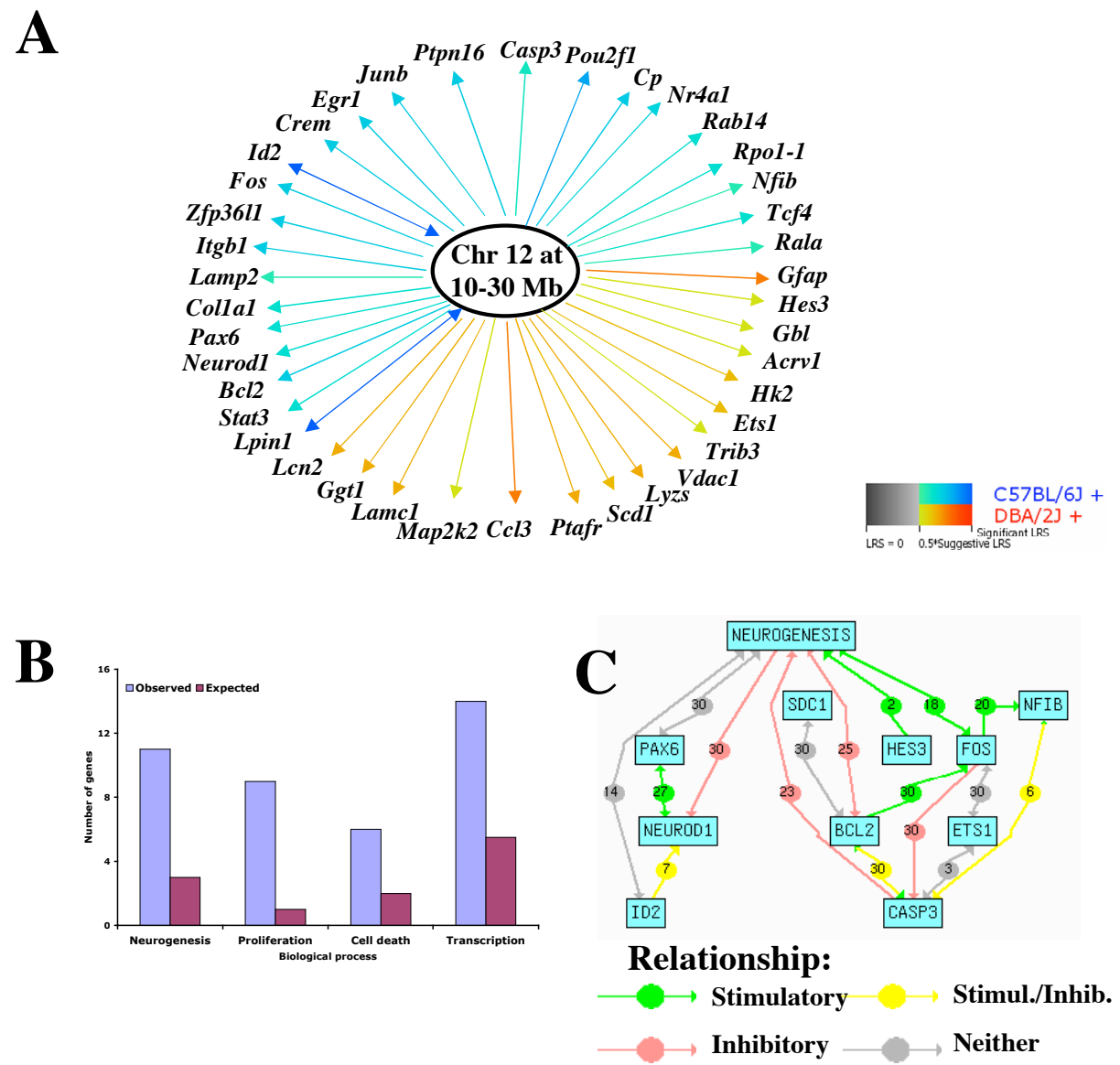

Figure 4.2. Chromosome 12 locus modulates transcription, differentiation, proliferation, and apoptotic mechanisms. A: Genetic networks were derived from transcripts sharing regulatory loci as shown in Figure 4.1. Blue lines connecting specific genes to loci represent correlations for elevated gene expression in mice with $\mathrm{C} 57 \mathrm{BL} / 6$ allele, and orange lines represent correlation for elevated gene expression in mice with DBA/2J allele. Genes located within the regulatory loci (cis-regulated) are indicated with a two-arrow line. B: The major functional themes described by the network's gene functions are the regulation of transcription, differentiation, proliferation, and cell death. A non-biased, statistical approach to defining the function of the network $(n=41$ genes) is to compare the observed number of regulated genes as compared to the expected number in a population belonging to a particular functional category. For the network we observed $27 \%$ (11 out of 41 genes) of genes to be related to the regulation of neural development and differentiation. This percentage is higher than the percentage (7\%) observed among the total population of retinal reactive transcripts, and much higher than the percentage of expected genes in the entire genome. To determine functional themes within a network of genes, we retrieved the Gene Ontology annotation data using WebGestalt (WEB-based GEne SeT AnaLysis Toolkit; http://genereg.ornl.gov/webgestalt). C: We queried the biological literature using text-mining tools to illustrate networks within the transcripts grouped into the neurogenesis category (Pax6, Neurod1, Id2, Nfib, Egr1, Hes3, Stat3, Ets1, Sox11, Casp3, Itgb1, and Sdc1). The literature documents known molecular interactions these genes, including activation and inhibition that occur during neurogenesis. To mine literature abstracts we used the online tool Chilibot [www.chilibot.net]. 
known molecular interactions such as activation and inhibition that occur during neurogenesis (Figure 4.2A). These literature-based associations illustrate that transcription factor NeuroD1 activates pro-neural transcription factor Pax6. The network also shows that transcription repressor ID2 modulates NeuroD1. All these relationship are well established during neurogenesis. Additional content-rich networks can be derived for transcripts involved in the regulation of transcription, cell cycle, and cell death. Text-mining approaches support our hypothesis and provide direction of potential molecular interactions. Data mining of biological concepts, gene function, and protein interactions suggests that the chromosome 12 network may regulate transcription, proliferation, apoptosis, and changes in phenotype (that is, de-differentiation) during retinal wound healing.

\section{Candidate Upstream Modulators}

Having determined the network's targets and biological significance, the next level of analysis defines the upstream modulator responsible for the chromosome 12 locus. The correlation of reactive genes to the interval on chromosome $12(10$ to $30 \mathrm{Mb})$ suggests that within the locus there are one or more polymorphic genes controlling the expression of genes in this network. This chromosomal interval has a high degree of homology (that is, synteny) with intervals on the human chromosome 2 ( 1 to $18 \mathrm{Mb}$ or $\mathrm{p} 25$ to $\mathrm{p} 24.3$ ) and on the rat chromosome 6 (34 to $48 \mathrm{Mb}$ or q14 to q16) [NCBI MapViewer, http://www.ncbi.nlm.nih.gov/mapview]. In these syntenic regions, the gene order and gene homology is highly conserved. Within the locus there are over sixty known genes (Figure 4.3A). A review of how this network was derived is helpful in determining the search criteria for candidate genes. The network was derived from transcripts that are differentially expressed in the retina after trauma and from transcripts that are co-regulated in mouse forebrains due to genetic differences between the C57BL/6 and DBA/2J mouse strains. Consequently, a candidate gene must have genetic polymorphisms that result in the expression variability of its own transcript (self-regulation). In addition, the candidate gene's function must be consistent with molecular events that occur during retinal wound healing. Our analyses to 
Figure 4.3. Candidate genes for chromosome 12 network. A candidate gene must have genetic polymorphisms that result in the expression variability of its own transcript (cis-regulation). In addition, the candidate gene's function must be consistent with molecular events that occur during retinal wound healing. A: Within the locus there are over sixty positional genes. This diagram summarizes our computational work to examine each gene's single nucleotide polymorphism density; self-regulation in CNS tissues; gene expression pattern during the developing retina and injured retina; and known function in CNS pathology and development. Within the zoomed interval, bars represent genes and their spacing represents approximate location. Red bars represent genes that meet criteria. Genes location was determined using Genome Browser [http://genome.ucsc.edu, Mouse May 2004 assembly]. B: Polymorphisms for Id2 and Lpin1. The C57BL/6 and DBA/2J sequences for Id 2 display four genetic variants and for Lpin1 over140 variants. As a complementing guide to defining gene variants that are responsible for susceptibility to CNS injury, we compared the gene sequences of the DBA/2J to sequences from two resistant strains, the C57BL/6 and 129/svj [Schauwecker and Steward, 1997; Inman et al., 2002]. This approach identified three nucleotides in the Lpin1 sequence of the DBA/2J that are different from sequences of both the C57BL/6 and 129/svj (Celera SNP IDs mCV22346966, mCV22347703, and mCV22347384). Single nucleotide polymorphisms (SNPs) were identified using the Celera SNP database available through the SNP Browser tool in GeneNetwork. C: We identified genes whose expression patterns in forebrain, cerebellum, and striatum of BXD mouse strains map to their gene locations. Within the interval we identified in red genes that appear to self-regulate their own expression in at least two brain regions. Linkage maps were generated using the Interval Mapping and Cluster Tree tools at GeneNetwork (UTHSC Brain mRNA U74Av2 HWT1PM, December 2003; GNF Hematopoietic Cells U74Av2 RMA, March 2004; HBP/Rosen Striatum M430V2 RMA, April 2005; SJUT Cerebellum mRNA M430 RMA, March 2005). D. We determined genes that are differentially expressed during the developing retina and injured retina (in red). Gene expression patterns in the developing mouse retina were obtained using Retina Developmental Gene Expression [www.scripps.edu/cb/friedlander/gene_expression/]. Gene expression patterns in the injured rat retina were obtained using our data available online at Gene Expression Omnibus (GEO, www.ncbi.hlm.nih.gov) as the group series GSE1001. Gene expression levels from cultured mouse neurons [Kraft et al., 2004], mouse astrocytes [Kraft et al., 2004], rat Muller cells [Gerhardinger et al., 2005], human optic nerve astrocytes [Yang et al., 2004], human U87 glioma cells [unpublished data, Eldon Geisert], and rat RPE cells [unpublished data, Eldon Geisert] were obtained from published and unpublished data. Asterisk represent genes with no probe set available. We also examined if any of the genes in the locus were involved in gliosis, neurodegeneration, neurogenesis in the CNS using the text-mining tool Chilibot [www.chilibot.net/]. We used the following Affymetrix probe sets: for Id2 were U74v2 93103_at, M430 1435176_a_at_A, and U34A rc_AI230256_at; for Lpin1 U74v2 98892_at, and M430 1426516_a_at_A; for Sox11 U74v2 101631_at and M430 1431225_at_B, and M430 1453002_at_B; and for AL024210 M430 1438758_at_A and M430 1449076_x_at_A. AL024210 is highly homologous to human MTCBPl (NP_060739) and rat Alpl (NP_954528). 


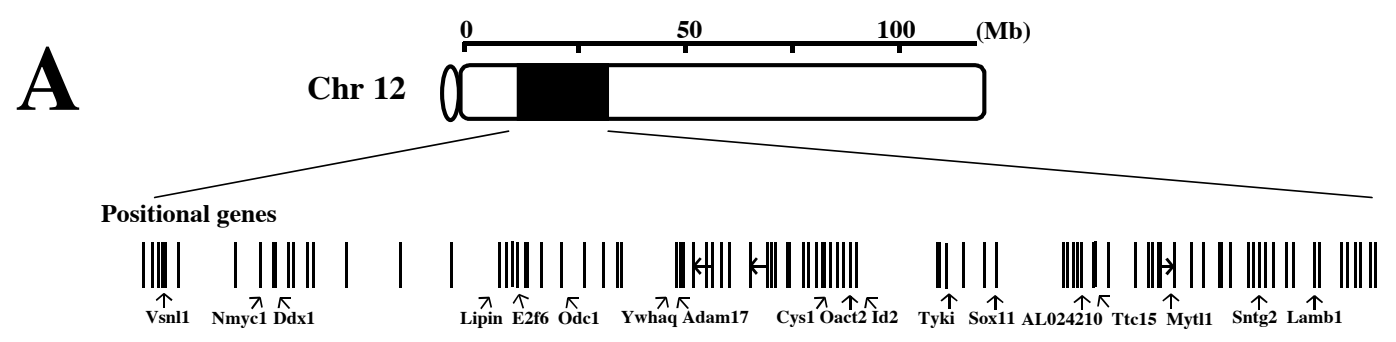

cis-regulation in CNS tissues

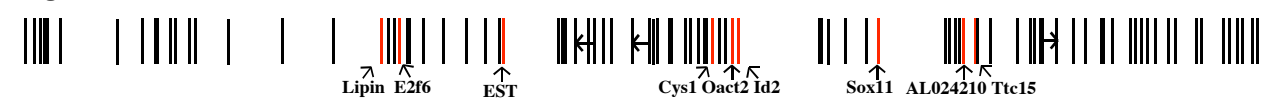

CNS pathology/development

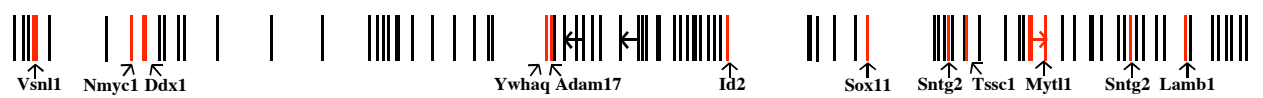

Differentially expressed in injured/developing retina

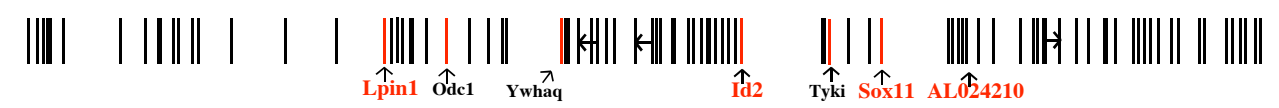

B

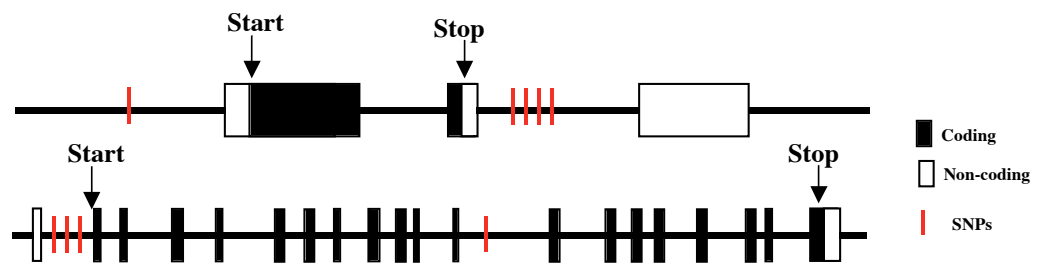

1
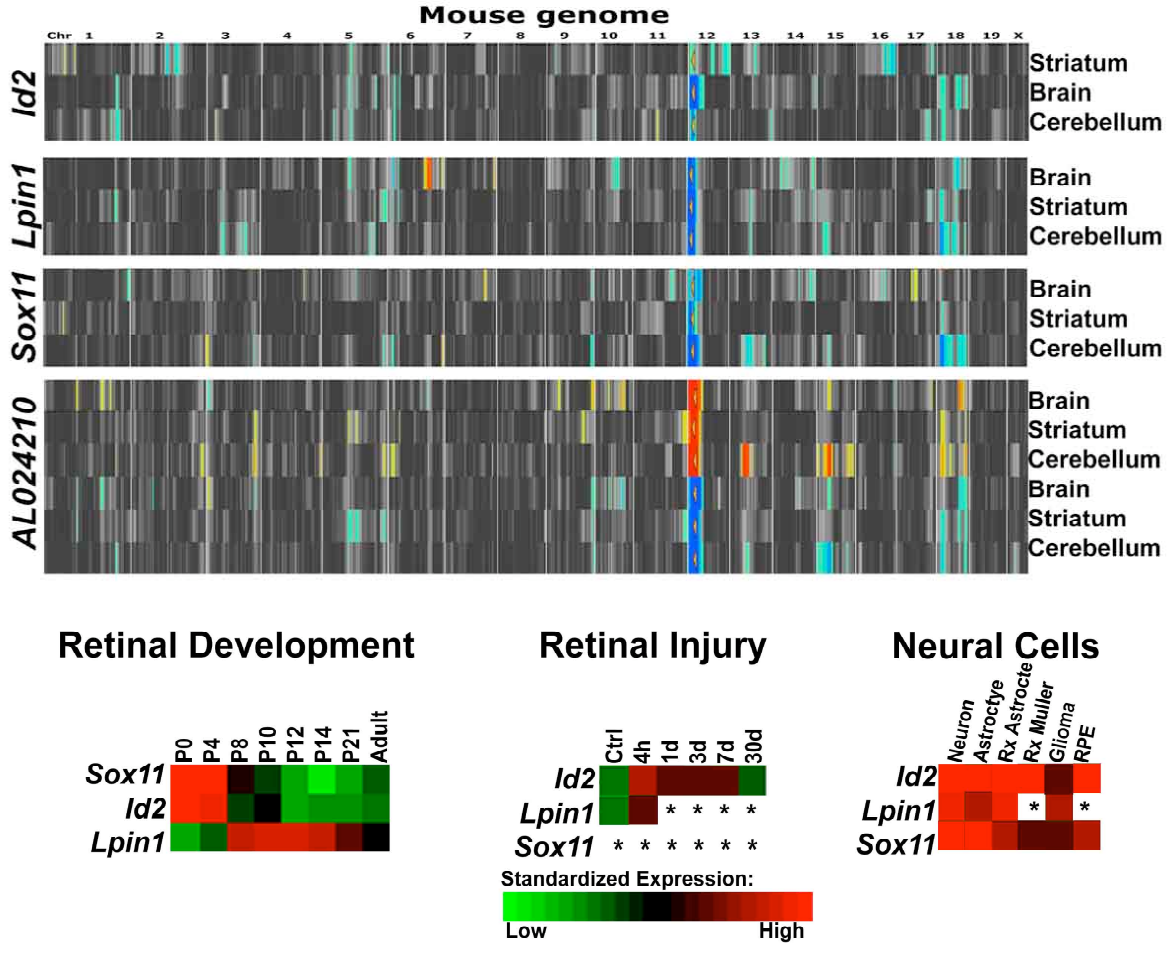
identify the upstream regulator for the network includes expression profiles from injured retina, brain, and spinal cord; gene expression genetics of mouse forebrain, cerebellum, and striatum; as well as, gene expression atlases and text-mining of biological literature.

We identified genes whose expression patterns in forebrain, cerebellum, and striatum of BXD mouse strains map to their gene locations. Using this criterion, we identified the known genes Lpin1, E2f6, Cys1, Oact2, Id2, and Sox11, as well as the predicted genes AL024210 and Tct15 as polymorphic genes that display significant self-regulation of their transcript expression in at least two CNS tissues. For example, Lpin1, Sox11, AL024210, and Id2 have their gene location on chromosome 12 at 15.9, 23.9, 25.2, and 21.6, respectively. Their DNA sequences between the C57BL/6 and DBA/2J genomes display single-nucleotide polymorphisms (SNPs) in their coding and regulatory regions (Figure 4.3B, Lpin1 and Id2 SNPs only shown). Lpin1 has over140 SNPs within the transcribed regions most of them located within introns. Within the exons there are missense mutations (Celera SNP ID mCV22640829) and several silent point mutations (Celera SNP IDs mCV22640556-7). Sox11 has a missense mutation (Celera SNP ID mCV22347029) and several silent point mutations (Celera SNP IDs mCV22345285-7030). AL024210 has SNPs in the first intron (Celera SNP ID mCV23344577) and in the 5' untranslated region (Celera SNP IDs mCV23344575-6). Id2 also has SNPs on its promoter (Celera SNP ID mC22302957), introns (Celera SNP IDs mCV22302969, 77-79, 89), and third exon (mCV22302990, 2991, 3002, 3003). These polymorphisms might explain the strong linkage of expression patterns of Lpin1, Sox 11, AL024210, and Id2 to their gene locations (cis-regulation). The self-regulatory role of these genes extends across brain tissues including forebrain, cerebellum, and striatum (Figure 4.3C). To further explain this self-regulatory function, we examined if the polymorphisms can alter functional motifs or splice sites. For example, Id2 has a SNP on the promoter (Celera SNP ID mC22302957) that is adjacent to a putative nuclear factor Y (NF-Y) transcription factor-binding site [Vazquez et al., 2005]. Lpinl has exonic SNPs near the 5'splice site of intron 11 (Celera SNP ID mCV22346966) and near two spliced mouse expressed sequenced tags on exon 1 (Celera SNP IDs mCV22347703 and mCV22347384). Genetic variants 
in a transcription factor binding site and at splice locations can lead to difference in mRNA levels. These differences make the genetic mapping possible. Of interest to CNS wound healing are three Lpin1 SNPs in DBA/2J sequence. They are different to the nucleotides in C57BL/6 and $129 /$ svj sequences. These two latter strains are known to be resistant to trauma-induced cell death in brain and spinal cord [Schauwecker and Steward, 1997; Inman et al., 2002]. In silico analysis of SNPs is valuable in defining candidate genes and predicting potential mechanism by which the SNPs interfere with the expression of candidate genes.

The role of a gene as candidate regulator of chromosome 12 network, and potentially of retinal wound healing, is bolstered by determining if its gene product is known to play a role CNS development and disease. Using the publicly available data on mouse retinal development [Dorell et al., 2004], we determined that Lpin1, Id2, and Sox11 are expressed by the developing retina and have well defined patterns of expression (Figure 4.3C; note that $A L 024210$ levels were below detectable thresholds). Using our microarray and RT-PCR data on rat retinal injury [Vazquez, et al., 2004], we found that Lpin1 and Id2 are acute phase genes during retinal injury (Figure 4.3C; the Sox11 U34 probe set displayed signals below noise thresholds and there is no probe set for AL024210). Lpin1, Id2, and Sox11 can be expressed by cultured neural cells including quiescent cortical neurons and astrocytes, quiescent retinal pigment epithelium. Glial cells cultured from reactive optic nerve head and diabetic retinas also expressed Id 2 and Lpin1 at high levels of expression, and Sox11 at low to moderate levels of expression. Together these results document that Lpin1, Id2, and Sox11 are candidate genes because they display (1) selfregulation in the brain, cerebellum, and striatum; (2) polymorphisms between parental strains; (3) differential expression during the developing retina and/or injured retina; and (4) moderate to high levels of expression in normal and reactive neural cells. However, we cannot exclude the role of predicted genes (AL024210 and Tct15), or an unknown gene within the locus as potential regulators of chromosome 12 network. As a first step toward documented the effect of the chromosome 12 locus on wound healing events, we focus on the candidate genes Id2 and Lpin1. 


\section{Chromosome 12 Network Modulates Retinal Wound Healing}

Our work has led to the hypothesis that the $10-30 \mathrm{Mb}$ locus on chromosome 12 modulates wound healing in the CNS. The segregating pattern of the C57BL/6 and DBA/2J alleles for the chromosome 12 locus in BXD RI strain background generate a natural range of transcript expression (low to high) for candidate genes. We utilize this system of gene expression dosage to test hypotheses and to further define expression relationships within the network. We specifically focus on gene dosage effects of candidate genes Id2 and LpinI on the expression of acute phase genes Fos and Stat3, apoptosis gene Casp3, gliosis genes Gfap and Cd81, and survival gene Crygd.

We confirmed dose levels for candidate genes Id2 and Lpin1 in retinas from BXD RI strains at the transcript and protein level. In forebrains of BXD RI mouse strains, normal Id2 and Lpin1 transcript levels are highly variable $\left(\mathrm{F}_{34,99}=5.2\right.$ and $\mathrm{F}_{34,99}=5.1, \mathrm{p}<10^{-9}$; Figure 4.4A, expression pattern for $I d 2$ shown). C57BL/6 forebrains expressed higher levels of $I d 2$ and LpinI than DBA/2J forebrains, whereas, the F1cross displayed Id2 and Lpin1 levels between those of the parental strains. In BXD RI strain forebrains, high Id2 and LpinI levels correlate with the segregating pattern of the C57BL/6 allele, whereas, low Id2 and Lpin1 levels correlate with the DBA/2J allele. Similar patterns of Id2 and Lpin 1 variability was observed in the striatum and cerebellum of BXD RI mouse strains [GeneNetwork, probe sets 1435176_a_at_A and 1426516_a_at_A]. To test this relationship in the retina, we measured transcript levels at both ends of the Id2/Lpin1 expression spectrum. For a high Id2/Lpin1 expresser we used the BXD38 strain. For the low Id2/Lpin1 expresser we used the DBA/2J mouse strain. Qualitative RT-PCR confirmed that the BXD38 retina expressed higher transcript levels of Id2 and Lpin1 than DBA/2J retina ( $p<0.05$; Figure 4.4B). We also confirmed the protein levels for ID2 in selected BXD RI mouse strains. Protein levels documented that ID2 protein levels in BXD38 and C57BL/6 retinal samples were higher than in DBA/2J retinal samples, and the protein levels for F1 cross were in between those of the parental stains (Figure 4.4B). In protein samples we also noted low variability within strain samples; but in general the samples confirmed the ID2 dosage predicted by the microarray analysis of BXD RI strain forebrains. Protein levels were not 

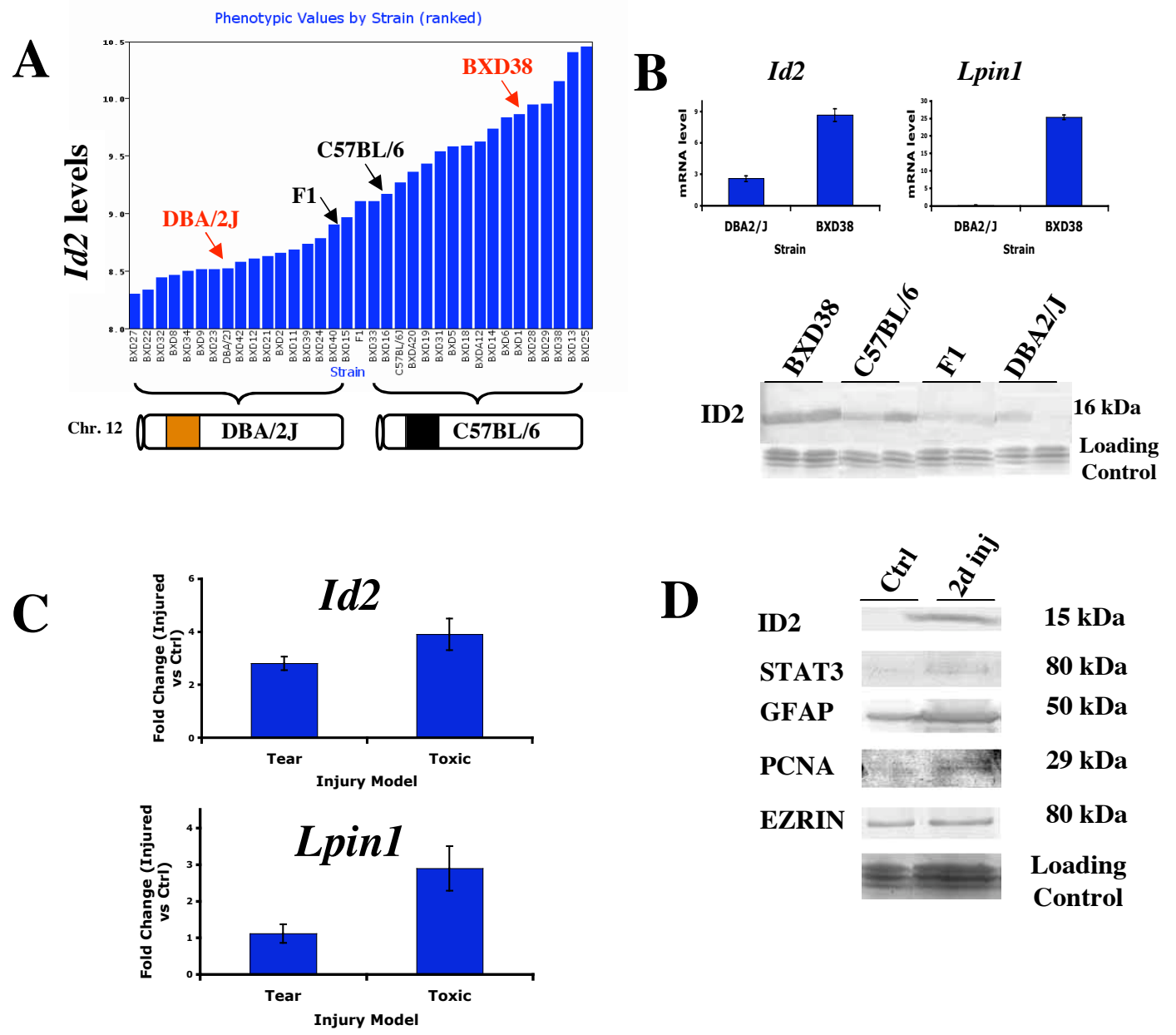

Figure 4.4. Id 2 and Lpin1 are differentially expressed in response to genetic differences and trauma. A: Gene expression pattern for $I d 2$ across forebrains from BXD recombinant mouse strains. Id 2 expression is highly heritable $\left(\mathrm{F}_{34,99}=5.2, \mathrm{p}<10^{-9}\right)$. High $I d 2$ expression levels correlated with the C57BL/6 allele of the chromosome 12 locus (at 10-30 Mb); whereas, low Id2 expression levels correlated with the DBA/2J allele. Similar results were also obtained for Lpin I $\left(\mathrm{F}_{34.99}=5.1, \mathrm{p}<10^{-9}\right.$; data not shown). Data obtained from GeneNetwork [UTHSC Brain mRNA U74Av2 HWT1PM, December 2003]. B: Transcripts levels for Id2 and Lpin1 in the retina of BXD RI strains were measured with real time RT-PC. For a high Id2/Lpin1 expresser we used the BXD38 strain. For the low Id2/Lpinl expresser we used the DBA/2J mouse strain. We also confirmed the protein levels for ID2 in selected BXD RI mouse strains. Protein levels were not measured for Lpin1. C: Transcripts levels for Id2 and Lpin1 after retinal trauma. Transcripts were measured in DBA/2J retina four hours after a mechanical tear or vitreal injections of kanaic acid. D: Protein levels from injured retina also confirmed the upregulation of ID2. The upregulation of ID2 in injured retina is consistent with the upregulation of classic wound healing factors such as the acute phase factor STAT3, the reactive gliosis marker GFAP, and the proliferative maker PCNA. Ezrin (villin 2) is a house-keeping gene product. For each protein and RNA sample, retinas from two normal or injured animals were pooled ( $n=4$ retinas per sample). 
measured for Lpin1. These results suggest BXD38 retina expresses higher levels of Id2 and Lpin1 than DBA/2J retina.

We also confirmed the response of $I d 2$ and Lpin1 to retinal trauma. Microarray analyses revealed that rat retina, brain, and spinal cord upregulate $I d 2$ after a mechanical injury [Vazquez et al., 2005]. In the rat retina, $I d 2$ is upregulated during the acute phase (6 fold change, $\mathrm{p}<0.03$; 4 hours after injury). During the delayed acute and late chronic phase $I d 2$ is also upregulated but at lower levels ( 3 fold change, $\mathrm{p}<0.05 ; 7$ hours after injury). The rat U34 Affymetrix array does not have a Lpin 1 probe set. Here we measured Id2 and Lpinl upregulation in mouse retina after different models of retinal trauma. Id2 is upregulated as an acute phase gene in response to a retinal tear and toxic injury in DBA/2J retina $(2.8 \pm 0.2$ and $3.9 \pm 0.3$ fold changes, $\mathrm{p}<0.05$; Figure 4.4C). Similar changes were observed in the injured BXD38 retina (data not shown). Lpin1 is

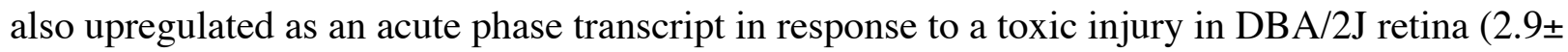
0.25 fold changes, $\mathrm{p}<0.05$; Figure $4.4 \mathrm{C})$. No significant changes were observed for Lpin1 in the injured BXD38 retina (data not shown). Protein levels from injured retina also confirmed the upregulation of ID2 (Figure 4.4D). The upregulation of ID2 in injured retina is consistent with the upregulation of classic wound healing factors such as the acute phase factor STAT3, the reactive gliosis marker GFAP, and the proliferative maker PCNA (Figure 4.4D). These results confirm that $I d 2$ and Lpin 1 are acute phase genes of the injured mouse retina.

To investigate the role of chromosome 12 locus during retinal injury, we examined the wound-healing response of retinas that expresses high levels of Id2/Lpin1 (strains with the C57BL/6 allele) and compared this to low levels of Id2/Lpinl (strains with the DBA/2J allele). We induced retinal wound healing by exposing the retina with kanaic acid, which results in glutamate-induced neurotoxicity. The neurotoxin induced significant upregulation of candidate genes and of retinal markers of acute phase, gliosis, apoptosis, and survival in BXD38 and DBA/2J retina (Figure 4.5A). Expression differences between strains were measured by a ratio with the injured BXD38 retina as the numerator and injured DBA/2J retina as the denominator. We observed distinct expression differentials for candidate genes, wound-healing genes, and 

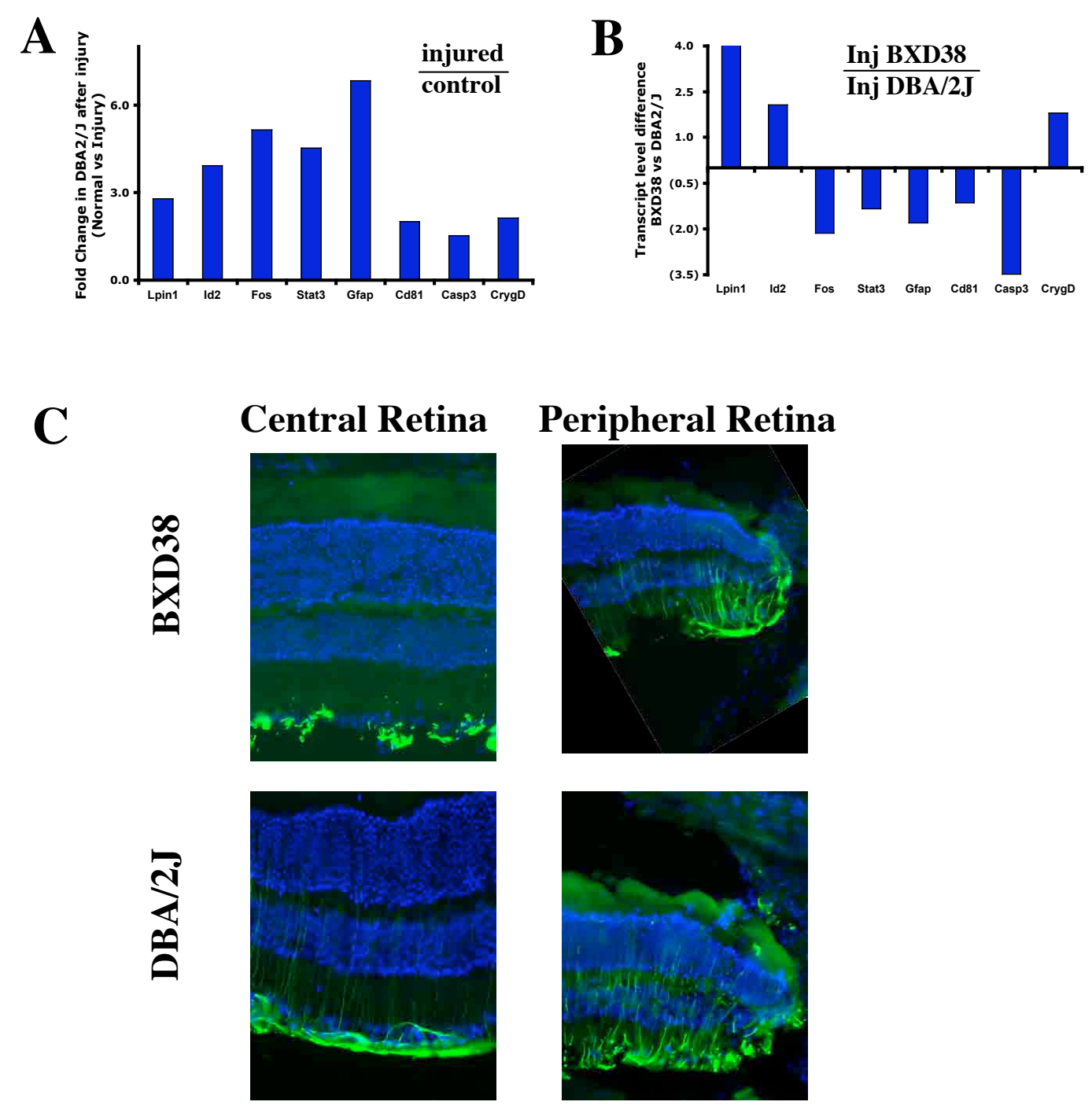

Figure 4.5. Higher levels of $I d 2$ and Lpin 1 correlated with lower retinal stress markers. To investigate the role of chromosome 12 locus during retinal injury, we examined the wound-healing response of retinas that expresses high levels of Id2/Lpinl (BXD38 strain with the C57BL/6 allele) and compared this to low levels of Id2/Lpinl (strains with the DBA/2J allele). A: Acutephase levels for markers of acute phase (Fos and Stat3), gliosis (Stat3, Gfap, and Cd81), apoptosis (Casp3), and survival (Crygd) were measured in BXD38 and DBA/2J retinas of fours after a vitreal injection of kanaic acid. Data for DBA/2J retina shown. B: Expression differences between strains were measured by a ratio with the injured BXD38 retina as the numerator and injured DBA/2J retina as the denominator. For each RNA sample, retinas from two injured animals were pooled ( $\mathrm{n}=4$ retinas per sample). C: We extended these findings by using an optic nerve crush model, a retinal injury that produces a slow progressing wound-healing response. Six days after an optic nerve crush, we measured stress levels using the classic stress marker GFAP, a cytoskeletal protein normally expressed by astrocytes and the end-feet of Muller cells ( 2 animals per strain). 
survival genes (Figure 4.5B). Candidate genes Id2 and Lpinl were expressed more highly in the injured BXD38 retina than in the injured DBA/2J retina $(2.1 \pm 0.5$ and $144 \pm 2.1$ fold differences, $\mathrm{p}<0.05)$. Expression of acute phase genes Fos and Stat3, apoptosis gene Casp3, gliosis genes Gfap and $C d 81$ were lower in the injured BXD38 retina than in the injured DBA/2J retina (Figure 4.5B). As a measure of neuroprotection, we surveyed the transcript levels of the chaperone crystallin gamma (Crygd). The high Id2/Lpin1 expresser strain (strains with the C57BL/6 allele) displayed higher transcript levels of the survival gene Crygd (Figure 4.5B). Our comparison of wound-healing gene expression in the high and low Id2/Lpin1 expressers, suggest that higher levels of Id2/Lpin1 expression and the C57BL/6 allele correlate with lower levels of acute phase, gliosis, and apoptosis markers. Higher levels of Id2/Lpin1 expression and the C57BL/6 allele also correlated with increased expression of survival gene Crygd.

We extended these findings by using an optic nerve crush model, a retinal injury that produces a slow progressing wound-healing response. An optic nerve crush results in a woundhealing process that involves the death of retinal ganglion cells and activation of Muller cells [Wax and Tezel, 2002]. We measured levels of stress using the classic stress marker GFAP, a cytoskeletal protein normally expressed by astrocytes and the end-feet of Muller cells (2 animals per strain). Six days after an optic nerve crush, the central and the peripheral retina of the low Id2/Lpin1 expresser (DBA/2J strain) displayed increased GFAP immunoreactivity: Muller cells increased the expression of GFAP, as seen by the increased immunoreactivity along the radial branches of Muller cells (Figure 4.5C). In contrast the high Id2/Lpin1 expresser (the BXD38 strain) at six days after injury displayed GFAP labeling of Muller cells only at the peripheral retina and a delayed upregulation of GFAP in the central retina. The differences in GFAP immunoreactivity between the high and low Id2/Lpin1 expressers (Figure 4.5C) were consistent with the mRNA results (Figure 4.5B): higher levels of Id2/Lpin1 (the C57BL/6 allele) confer a decrease reactive response by Muller cells. These results suggest that the C57BL/6 allele may confer resistance to retinal injury whereas the DBA/2J allele may confer susceptibility. 


\section{Id2 Silencing Decreases Wound Healing Response of Cultured Astrocytes}

Candidate upstream modulator ID2 is known to be involved in proliferation and differentiation of neural precursor cells [Yokota and Mori, 2002]. In CNS pathology, ID2 also has been described in reactive glia [Tzeng et al., 1999; Tzeng et al., 2001; Aronica et al., 2001; Hernandez et al., 2002]. We investigated the function of ID2 in glial cells by silencing the $I d 2$ transcript in a mouse astrocyte cell line, the C8D1A astrocytes. C8D1A astrocytes efficiently uptake short inhibitor RNAs (siRNAs) and transport them near the nucleus (Figure 4.6A, red channel). We designed three siRNAs to target the Id2 mRNA (Figure 4.6B) and compared their silencing efficiency against a scrambled siRNA. Off-target silencing was determined by measuring the transcript and protein changes of the reactive gliosis molecule, CD81. After a $24 \mathrm{~h}$ transfection, the siRNAs successfully reduced $I d 2$ transcript to levels below $30 \%$. The siRNA with the best silencing efficiency was used for all experiments (93\% silencing, $p<0.001$; Figure 4.6B). This siRNA targets the 3' untranslated region of Id 2 at about 568 nucleotides from the starting site. Id 2 siRNAs produced minimal off-target effects, as shown by the non-significant changes in $C d 81$ transcript levels (Figure 4.6B). Reductions in $I d 2$ expression also resulted in a 33\% decrease in ID2 protein levels to $(p<0.02$, Figure 4.6C). Protein change for off-target genes was minimal. CD81 protein levels were virtually unchanged (Figure 4.6C). Therefore, our RNA interference technique successfully decreased the expression of $I d 2$ with minimal off-target effects.

To determine functional changes due to lower ID2 levels, we used a cell culture model of wound healing induced by the loss of contact inhibition. In this model, astrocytes at the leading edge upregulate GFAP, migrate, and proliferate to repair the wound. Twenty-four hours after transfection, we induced the loss of contact inhibition by scrapping cells off at the center of the monolayer. We followed morphological changes of the reactive astrocytes entering the wound using time-lapse photography. At 24 and $48 \mathrm{~h}$ there was a significant decrease in the rate of wound-healing closure (Figure 4.6D). For example, migration distance of the $I d 2$-siRNA treated cells was $54.8 \%\left(\mathrm{p}<10^{-6}\right)$ of control-siRNA treated cells at $24 \mathrm{~h}$ after scratch (Figure 4.6D). To determine if the decrease in wound healing was due changes in cell cycle dynamics, we measured 


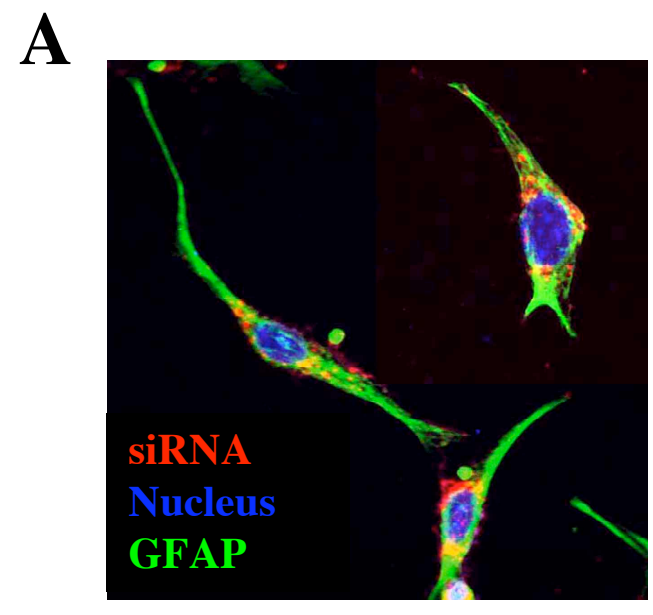

C
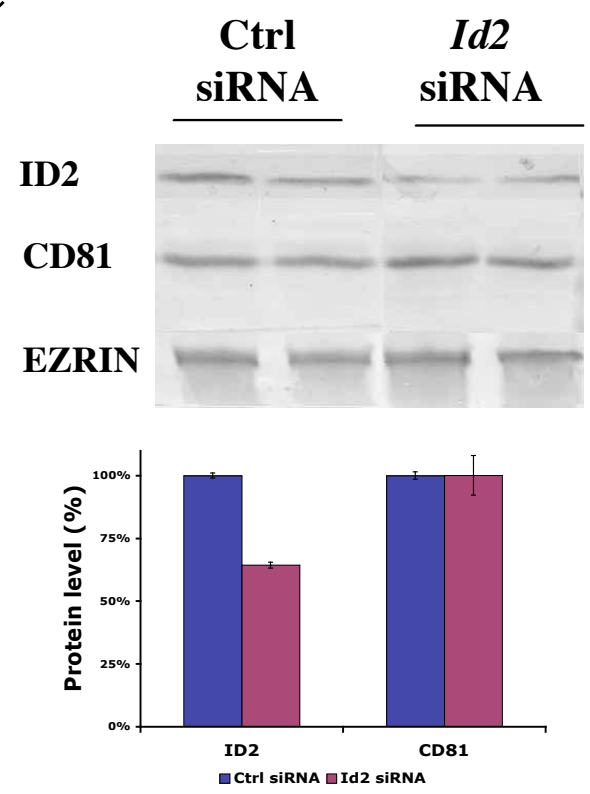

$\mathbf{E}$

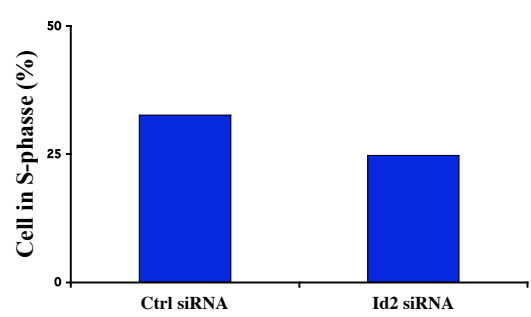

B
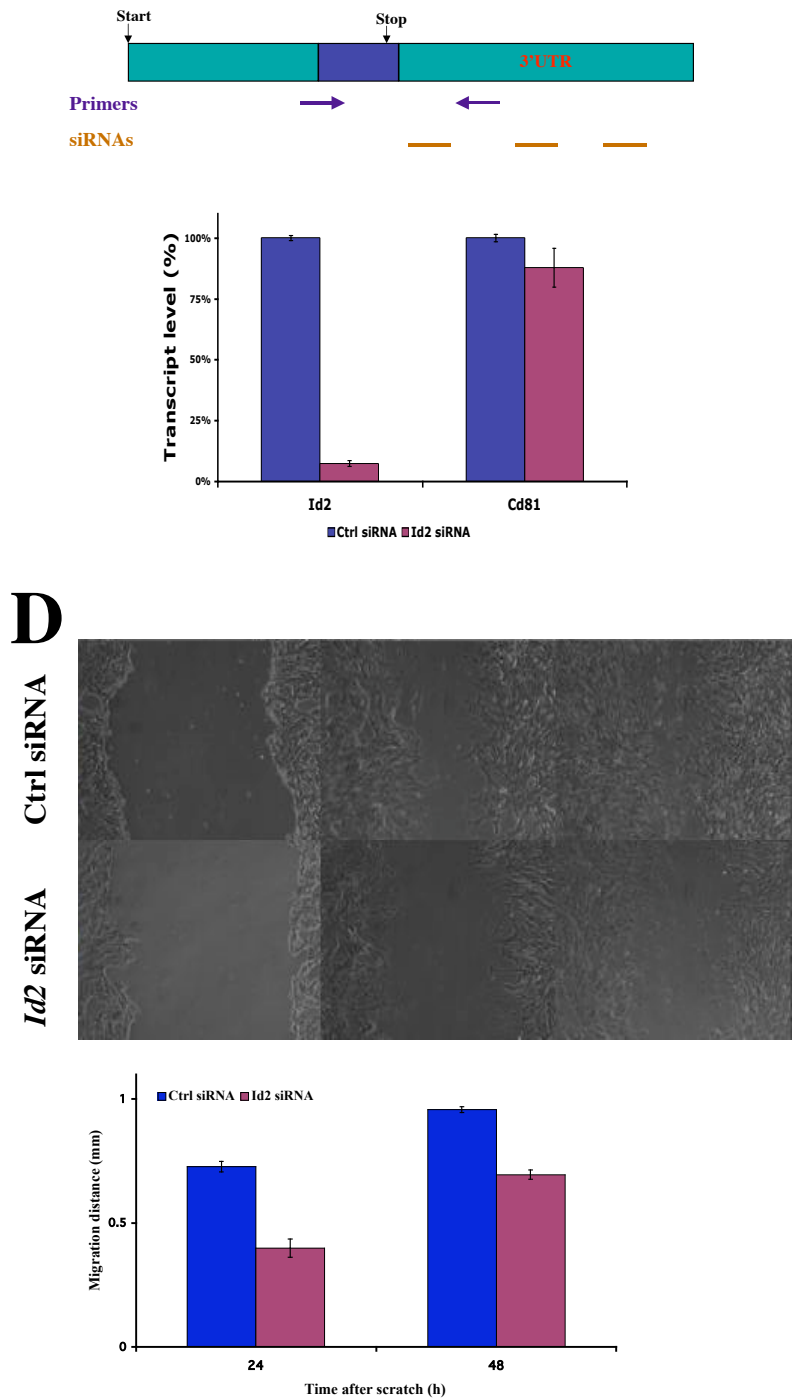

Figure 4.6. Id 2 silencing decreases the mitotic activity and healing response of cultured astrocytes. 
changes in mitotic rate using DNA cell cycle analysis. DNA content measurements with flow cytometry revealed that there was a $32.5 \%$ decrease in the number of cells in the S-phase (from 32.6 to $24.7 \%$ ). Together these data suggest that ID2 controls the progression of astrocytes through the cell cycle.

\section{Discussion}

The present study defined a genetic network and the location of its upstream modulator using gene expression profiling and bioinformatic tools. Our approach to defining networks controlling retinal wound healing involves (1) highlighting networks of highly co-expressed genes in different models of injury and different CNS tissues; (2) defining the location of an upstream regulator to a specific genomic locus using expression genetics; (3) predicting upstream modulators and targets using bioinformatic tools; and (4) testing the effect of candidate gene dosage on wound healing using animal and cell culture systems. Here we predict, refine, and test a genetic network that modulates the expression of genes involved in the early CNS wound-healing response. Upstream regulators for this network are located within the regulatory locus on chromosome 12,10 $30 \mathrm{Mb}$ away from centromere. Dosage levels for candidate genes within the locus (Id2 and Lpin1) correlated positively with lower expression levels for markers of acute phase response, gliosis, and apoptosis. Our results support the convergence of gene expression profiling, expression genetics, and bioinformatics as a rational approach to discover molecular pathways controlling retinal wound healing.

Previous studies identified three genetic networks that modulate the gene expression of wound healing genes [Vazquez et al., 2005]. Only one of these networks, chromosome 12 network, appears specific to the response of the CNS to injury. Our comparison of regulatory loci across tissues and functional groups documents that chromosome 12 locus specifically regulates the expression of wound healing genes in mouse brains. Our data documents that this network 
modulates genes known to regulate of transcription, differentiation, proliferation, and apoptosis of neural cells. Expression patterns of chromosome-12-modulated genes in the injured retina reveal that most of these genes are differentially expressed during the early acute and delayed subacute phases (Appendix C, Figure C.1). The function and temporal expression of the network's genes are consistent with early wound healing events in the injured retina. The early wound healing phases are characterized by neuronal cell death and glial proliferation at the site injury, as well as by the early activation of neuronal and glial cells leading to phenotypic changes (that is, de-differentiation). Published data also support the hypothesis that chromosome 12 network modulates the CNS response to trauma. In BXD RI mouse strains, the DBA2/J allele for the chromosome 12 locus is one of the loci associated with susceptibility to noised-induced cell death of spiral ganglion cells, the cells innervating auditory receptors cells [Willott and Erway, 1998]. The DBA/2J allele also predisposes susceptibility to generalized convulsions when exposed to intense auditory stimulation [Neumann and Collins, 1991]. In contrast, the C57BL/6 allele is one of the loci that confers enhanced neurogenesis and survival of new neurons and astrocytes in adult hippocampus [Kempermann and Gage, 2002]. Together these data suggest that the chromosome 12 network displays a prominent role in CNS wound healing.

To test the hypothesis that chromosome 12 network modulates the wound healing response in the retina, we examined the acute phase expression of classic immediate early genes Fos and Stat3, apoptotic gene Casp3, and reactive gliosis genes Stat3, Gfap, and Cd81 in one BXD RI strain segregating the C57BL/6 allele (BXD38 strain) and another segregating the DBA/2J allele (DBA/2J strain). The C57BL/6 allele correlated with low expression levels of genes involved in the immediate-early response, cell death, and reactive gliosis. The C57BL/6 allele also correlated with increased levels of the survival gene Crygd. In contrast, the DBA/2J allele correlated with higher levels of immediate-early response, cell death, and reactive gliosis genes, as well as, with lower levels of the survival genes. Our immunohistochemistry of injured retina confirmed that the $\mathrm{C} 57 \mathrm{BL} / 6$ allele and $\mathrm{DBA} / 2 \mathrm{~J}$ allele correlated with lower and greater levels of the classic marker of retinal stress, glial fibrillary acidic protein (GFAP). These data 
suggest that C57BL/6 allele confers protection and DBA/2J allele confers susceptibility to retinal damage. Our results are consistent with published data. The C57BL/6 background confers resistance to apoptosis and seizures in the auditory system; resistance to glutamate-induced neurotoxicity in brain and spinal cord; enhanced neurogenesis and survival of new neurons and astrocytes in hippocampus [Willott and Erway, 1998; Neumann and Collins, 1991; Kempermann and Gage, 2002; Schauwecker and Steward, 1997; Inman et al., 2002]. These data document that chromosome 12 locus can modulate the acute-phase expression of apoptotic, gliosis-related, and survival genes; and that within this locus there is a master regulator of the CNS response to injury.

A genetic variant located on chromosome 12 locus is responsible for the phenotypic differences in the wound healing response of BXD RI mouse strains. Using an integrated bioinformatic approach, we examined each polymorphic gene within the locus to determine the best candidate genes that can self-regulate its expression (that is, cis-acting) and regulate the expression of wound healing genes. Id2, Lpin1, Sox 11, and AL024210 displayed cis-acting regulation in brain, cerebellum, and striatum of BXD RI mouse strains. This means that a genetic variant (or variants) within or near the loci of these genes affect their own expression. Id2, Lpin1, Sox11, and AL024210 are candidate genes because they display highly conserved self-regulation across CNS tissues. Of these candidate genes, $I d 2$ is expressed by reactive glia in brain, spinal cord, and optic nerve head [Tzeng et al., 1999; Tzeng et al., 2001; Aronica et al., 2001; Hernandez et al., 2002]. $I d 2$ is also upregulated as an immediate early gene in injured retina, brain, and spinal cord [Vazquez-Chona et al., 2005]. Such strong circumstantial evidence is not available for Lpin1, Sox 11, and AL024210. As a complementing guide to defining gene variants that are responsible for susceptibility to CNS injury, we compared the gene sequences of the DBA/2J to sequences from two resistant strains, the C57BL/6 and 129/svj [Schauwecker and Steward, 1997; Inman et al., 2002]. This approach identified three nucleotides in the Lpin1 sequence of the DBA/2J that are different from sequences of both the C57BL/6 and 129/svj (Celera SNP IDs mCV22346966, mCV22347703, and mCV22347384). Our computational analyses of SNPs show that SNPs in Id2 and Lpin1 may affect DNA binding sites or splice variants. Using these approaches we fo- 
cused on transcription regulator Id2 and the nuclear protein Lpin 1 as the best, current candidate genes. Additional studies are required to determine if transcription factor Sox 11 and expressed sequence $A L 024210$ participate in early signaling of retinal wound healing. In normal and injured retinas, Id2 and Lpin1 were more highly expressed in strains carrying the C57BL6 allele than strains carrying the DBA/2J allele. Higher levels of Id2 and Lpin1 correlated with higher levels of survival gene Crygd and lower levels of acute-phase genes Fos and Stat3, apoptotic genes Casp3, and gliosis-related genes Gfap and Cd81. These results document that DNA variants on Id2 and Lpin1 of DBA/2J allele may result in lower expression of Id2 and Lpin1 and in higher levels of apoptotic genes and gliosis-related genes.

The known functions of ID2 are consistent with the potential roles of chromosome 12 during the early CNS response to injury: regulation of transcription, differentiation, proliferation, and apoptosis. In its classical function, ID2 modulates neural and glial differentiation via the negative regulation of basic helix-loop-helix (bHLH) transcription factors (for example, NeuroD and Olig) [Ross et al., 2003; Liu and Harlan, 2003; Wang et al., 2001]. ID2 binds to bHLH transcription factors to inhibit their binding to E-box sequences, defined by the CANNTG motif [Yokota and Mori, 2002]. E-boxes are present in promoters of many pro-neural differentiation genes (for example, $\mathrm{Pax} 6$ ) [Ross et al., 2003]. These data suggest that ID2 can modulate activation of retinal cells by regulating E-box mediated transcription. Several of the network genes have Eboxes in their promoters including Fos [Chaudhary and Skinner, 1999], Gfap [Tzeng, 2003], Sdc1 [Vihinen et al., 1996], Pax6 [Marsich et al., 2003], and $C p$ [Vazquez-Chona, personal in silico promoter observations]. For example, ID2 modulates the gene expression of Fos during Sertoli cell differentiation [Chaudhary and Skinner, 1999]. ID2 can also promote proliferation by inhibiting the E-box mediated transcription of cell cycle inhibitors (for example, p21CIP1/WAF1, p15INK4B, and p16INK4B) [Pagliuca et al., 2000]. Alternatively, ID2 may stimulate proliferation by binding to the unphosphorylated $\mathrm{Rb}$ protein, allowing the release of the transcription factor E2F. E2F in turn activates genes involved in G1-S phase transition and hence proliferation [Yokota and Mori, 2002]. In our studies, Id2 silencing in cultured astrocytes de- 
creased the number of cells entering the S phase. ID2 can also negatively modulate apoptosis. In animals with $I d 2$ null mutation, Sertoli cells and mammary epithelial cells display increased levels of apoptosis relative to wild-type animals [Yokota and Mori, 2002]. The increased apoptosis in Id2-/- animals suggest that ID2 may be required as a survival factor for some cell types [Yokota and Mori, 2002]. In our studies, higher levels of $I d 2$ correlated with higher levels of survival gene Crygd and lower levels of acute-phase genes Fos and Stat3, apoptotic gene Casp3, and gliosis-associated genes Gfap and Cd81. Together the functions of ID2 and these data suggest that ID2 is an ideal candidate upstream modulator of the chromosome 12 network.

The role of Lipin 1 during retinal wound healing is not clear. Lipin 1 belongs to a novel family of nuclear proteins that are involved in adipose tissue development and insulin resistance. Lipin proteins share three conserved domains: a lipin, N-terminal conserved region, a nuclear localization sequence, and a LNS (Lipin/Ned1/SMP2) conserved region. Mutations in the Lpin1 gene lead to fatty liver dystrophy (fld) in fld mice, characterized by loss of body fat, fatty liver, hypertriglyceridemia, and insulin resistance [Peterfy et al., 2001]. Lpin1 null mutations also lead to peripheral neuropathy due to the dysregulation of a battery of genes required for the regulation of storage lipid metabolism in both the endoneurium and peri/epineurium [Verheijen et al., 2003]. The mechanisms of action by lipin proteins are unknown. A study of yeast lipin protein, Ned1, revealed that lipin proteins can associate with factors involved in nuclear transport and chromosome segregation [Tange et al., 2002]. Gene expression patterns in the developing retina suggest that Lpin1 is expressed during the time of Muller cell birth and differentiation (P8-P14) [Retina Developmental Gene Expression, probe set 98892_at; Mouse Retina SAGE Library, Mm.153625]. We will use in situ hybridization to determine the cellular source of Lpin1 mRNA. We will also use fld mice to determine the role Lipin 1 has during wound healing.

Our work makes two clear contributions to the field of CNS wound healing research. At one level, our work describes an integrated approach of gene expression profiling and higherlevel bioinformatic analyses to define networks and their regulators. This approach is valuable because it suggests how researchers might develop a package of capabilities to enable a system- 
atic reconstruction of pathways related to their field. Our second contribution is the finding of a regulatory locus that modulates the early response of the retina to injury. Computational analyses and molecular manipulation of this locus will help define the regulatory gene(s) modulating the response of mammalian CNS to trauma and chronic stress. 
Chapter 5

Discussion 
This dissertation focused on understanding the response of the retina to injury. The response of the retina to injury is controlled by changes in gene expression and the series of studies presented in this dissertation are one of the first to use a unique combination of microarray studies to define loci controlling the initial events controlling the injury response. By implementing integrated gene expression profiling, expression genetics, and bioinformatics, we revealed three novel as-

pects of this wound healing process. First, groups of functionally related genes underlie the early, delayed, and sustained responses of wound healing (Chapter 2). Second, three specific genomic loci modulate coordinated changes in gene expression in mouse brains: regulatory loci on chromosomes 6, 12, and 14 (Chapter 3). Third, the chromosome 12 locus specifically modulates the expression of a group of genes involved in early wound-healing events. As a result of these studies we have identified two very good candidate genes Id2 and Lpin1 as potential modulators for the chromosome 12 network (Chapter 4). The chromosome 12 network represents one of the first regulatory mechanism derived from gene expression data of injured retina. Previous chapters discussed the biological significance of each of theses findings. In this chapter, I provide a retrospective discussion of the biological significance and scope of my experimental design to catalog global changes (microarrays), to define regulatory loci of gene expression (expression genetics), and to identify candidate genes (bioinformatics).

\section{Cataloging Global Changes During Wound Healing Using Microarrays}

\section{Global View of Gene Activity and Protein Expression}

At the most basic level, microarray analysis measures mRNA abundance. Traditionally, microarrays target the 3' regions of mRNA transcripts [Lipshutz et al., 1999]. Levels of hybridization to 3'regions are used to predict transcript expression levels and differences. We used real-time RTPCR as an independent means to confirm expression changes. Our results describe a strong agreement in direction of change between the Affymetrix microarray platform and the real-time 
RT-PCR method: $100 \%$ agreement for probes with high signals and $85 \%$ agreement for probes of all signal levels ( $\mathrm{n}=22$ genes) [Rogojina et al., 2003; Vazquez et al., 2004]. There is also a moderate correlation $(\mathrm{r}=0.76)$ when comparing the magnitude of changes [Vazquez et al., 2004]. Our results and other studies confirm the power of microarray to describe global gene expression changes in the injured retina [Vazquez et al., 2004; Chen et al., 2004; Yoshimura et al., 204; Wilson et al., 2003; Ahmed, et al., 2004; Gerhardinger et al., 2005; Rattner and Nathans, 2005].

We also confirmed the upregulation of protein changes predicted by our microarray study including GFAP, CD81, ID2, STAT3, as well as crystallins alpha, beta, and gamma (Figures 2.4 and 4.4). The literature provides additional support for protein expression changes for NFKB, CP, CLU, ANXA5, SPARC, and HSP27 [Wu et al., 2002; Wong et al., 2001; Strunnikova et al. 2001; Chen et al., 2003; Gilbert et al., 1999]. Our results demonstrate that changes in transcript levels correlate with immediate changes in protein levels. The silencing of Id2 using RNA interference decreased ID2 protein expression (Figure 4.6). Several studies integrated dynamic expression profiles of transcript and protein and found that the trends of differential expression are maintained across broad transcriptome and proteomic data [Hack, 2004; Beyer et al., 2004; Mijalski et al., 2005; Lin et al., 2004]. Lin and colleagues [2004] further demonstrated that changes in protein levels depend on gene expression changes. They showed that changes in protein levels that occur during the stress response are blocked if gene transcription is inhibited. However, we also found that the correlation between transcript and protein expression is weak to moderate [Rogojina et al., 2003]. When we decreased the $I d 2$ transcript by over $90 \%$, protein changes for ID2 decreased only by $33 \%$. These discrepancies in our study suggest that hybridization to 3'regions provide a limited picture of gene expression control after transcription and translation. Post-transcriptional control of gene expression includes the processing (for example, mRNA splicing), export, localization, turnover, and translation of mRNAs [Mata et al., 2005]. Studies that investigated alternative splicing of mRNA revealed that nearly $80 \%$ of the human genes have alternative isoforms [Johnson et al., 2003]. We conclude that differential splicing might explain the moderate correlation between our microarray and RT-PCR results. Post-translational events 
that could account for differences between our microarray and protein results include modification and degradation. Despite these limitations, microarray analysis was, and continues to be, our method of choice as it has a major advantage over current proteomic approaches. Proteomic approaches (two-dimensional gel and mass spectrometry) survey hundreds of gene products while microarrays can survey thousands of genes in a single experiment. In summary, commercial arrays provided a comprehensive, reliable assessment of global changes in the injured retina; however, the global microarray approach provided a qualitatively incomplete assessment of posttranscriptional and post-translational regulation of specific proteins of interest.

\section{The Challenge of Tissue Heterogeneity}

Transcriptome-wide analyses of injured retina are bulk characterizations of gene expression from diverse cell populations including photoreceptors, interneurons, ganglion cells, glial cells, peripheral blood cells, as well as their sub-populations. This type of cellular heterogeneity posses two major difficulties. First, cellular heterogeneity challenges the technical sensitivity of the system to detect transcripts expressed at low levels in unique cell populations. To enhance the detection and confidence level, we used comparative analyses of expression signatures across species and CNS tissues [Vazquez et al., 2005]. In our initial microarray study, Id2 was overlooked as a wound-healing gene due to the strict statistical criterion of a $98 \%$ confidence level. By highlighting common reactive transcripts in retina, brain, and spinal cord, we decreased technical and tissue specific noise and found that $I d 2$ is a common acute phase gene in CNS injury using a $95 \%$ confidence level.

The second issue raised by tissue heterogeneity is our ability to determine cell sources for specific changes. We determined a potential source by examining the expression of a transcript in profiles from cultured and dissociated CNS cells, including neurons, astrocytes, microglia, Muller glia, and RPE cells [Vazquez et al., 2005]. We found that often transcript expression and cellular source is maintained across tissues. For example, Gfap and Cd81 are expressed by astrocytes in the brain and in Muller glial cells in the retina [Clarke and Geisert, 1998; Gerhardinger et 
al., 2005]. However, making inferences about in vivo expression from cultured cell expression profiles must be made judiciously. Transcript expression is dynamic and certainly some transcript levels measured in these cells are in response to the trauma of dissociation and sorting. The identification of cell-specific genes is possible through the application of computational and genomewide analyses. Bryant Jones [2005] documented that genes involved in the regulation of glial cell development in Drosophila share a binding site for the glial cell missing (GCM) transcription factor. We predict that computational analyses of non-coding regions will predict the cellular source of gene expression. By combining our retinal transcriptome profiles with profiles of injured CNS tissue and cultured neuronal cells we reduced the challenges of tissue heterogeneity.

\section{Inferences About Changes in Function}

Changes in retinal function and regulatory mechanisms can be inferred from the grouping of genes with similar patterns of expression. We examined clustered genes based on their function described by the Gene Ontology database (www.geneontology.org) and from the literature. From these analyses, we determined that temporal clusters show significant enrichment in genes that are known to mediate similar functions (Figure 5.1) [Vazquez et al., 2004]. Transcription factors are expressed during the early response. Apoptosis and proliferation related genes are expressed during the delayed phase. Tissue remodeling genes and reactive gliosis are expressed during the chronic phase. Similar temporal patterns of functional groups were described in the woundhealing response of spinal cord and brain [Di Giovanni et al., 2003; Velardo et al., 2004; Natale et al., 2004]. The ultimate goal of gene expression profiling is to relate specific transcript changes with specific changes in cell function. Our results document that the silencing of $I d 2$ and $C d 81$ decreases astrocyte wound healing by different mechanisms: Id2 affects proliferation (Figure 4.6) and $C d 81$ affects migration (X. Wang and E. Geisert, personal observations). However, microarray analysis provided a limited view of the functional changes during retinal wound healing. We found that our microarray analyses described cultured RPE cells as mitoticly active on the basis of gene expression [Rogojina et al., 2003]. However, differences in proliferation rates between 


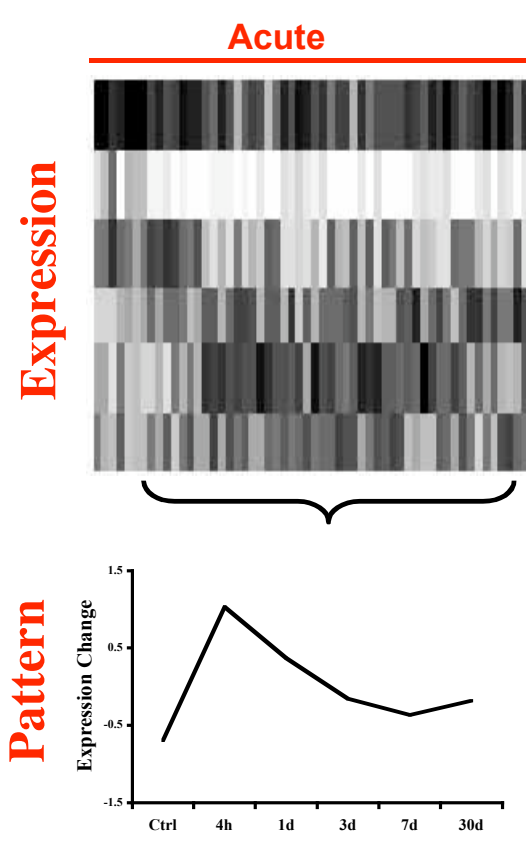

Transcription factors Egrl, Nr4a1, Fos, Junb, Nfkb1, Stat3, Cebpd, Irf1, Id2. प: Nfib, Nfkbia, Hes3

Inflammation-related COX-2, Il-1D, IL-6R

Glial activation pathways IL-6R $\square$ JAK $\square$ Stat 3 Jag2 $\square$ Notch $\square$ Nrap $\square$ Hes MAPK $\square$ Fos/Jun TNF $\square N F-K \square$

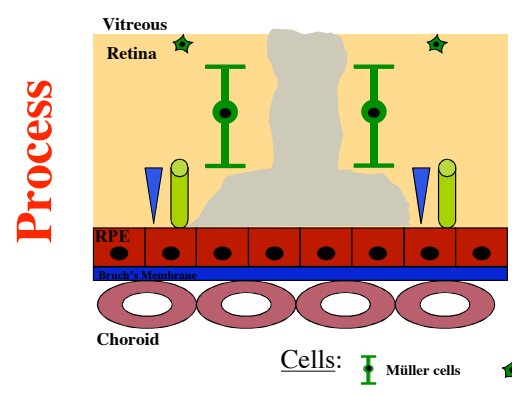

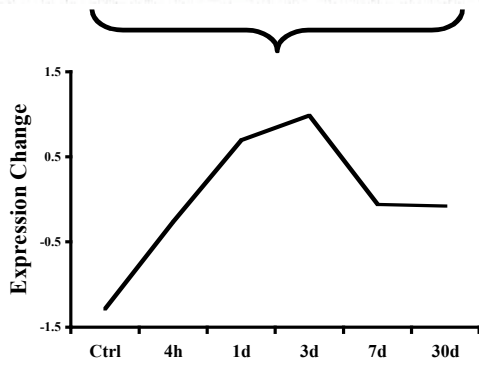

Cell growth Rbl2, Lcn2, Abi2, Arhgap, Btg2, Cd82, Dlgh1, Vhl, Ccnl, D123, Igf1, Igfals, Insig, 14-3-3, Cond1, Ccnd3 $\square$

Migration Cxcl10, Scyb13, Timp1, Plat, Arhgap, Cd48, Cd74, MHC I \& II

\section{Stress}

Snca, Hspala, Cp, Dnajb9, Mt1a

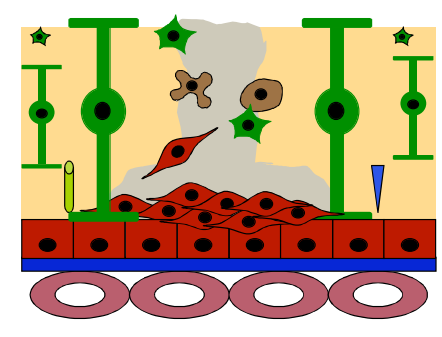

$\nabla 0$

if Microglia/macrophage

Tissue remodeling

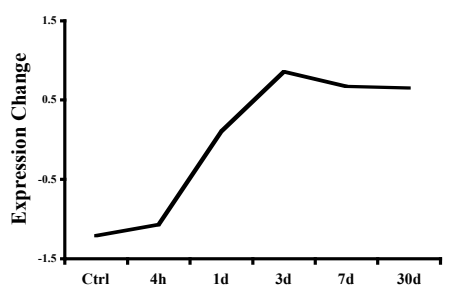

Gliosis

Gfap, Cntf, S100, Pea15, Pmp22, Clu, Cd81, Vcam1, Hsp27, Cryab, Cp, Apoe, A2m, Nfkb1, Stat3 Colla1, Sparc, Krt1-14, Bsp1, Tpm3, IMPG2, Tubb2

Crystallins

Cryaa, Cryab, Cryba, Crybb Crygc, Crygd, Cryge

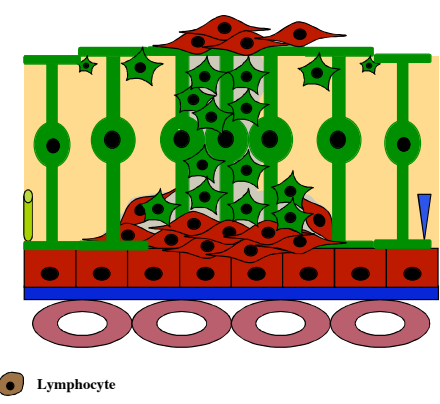

Figure 5.1. Gene expression after injury is temporally regulated and gene product functions are consistent with known the biology of retinal wound healing. 
RPE cell lines are indistinguishable based on gene expression. These results show that posttranslational events (phosphorylation, localization, modification and degradation) modulate cell function and that post-transcriptional regulation is not obvious from gene expression profiles. By providing global views of functional changes, our analysis of expression data is a first step toward the characterization of internal programs underlying retinal wound healing.

We predict that computational analyses will help define the molecular interactions defined by genes that share similar pattern of expression. For example, computational analyses of amino acid sequence analyses revealed that clustered genes share functional domains and display sites of known protein-protein interaction [Novatchkova and Eisenhaber, 2001]. These data suggest that gene clusters represent much more than abundance correlations. Indeed, Fraser and Marcotte [2004] argued that the complete set of relations defined by amino acid and DNA sequences within a cluster provides the most complete and unbiased analysis of gene function and regulation. By integrating gene expression data with additional levels of biological information

(for example, databases about gene function, functional motifs, and molecular pathways), we will infer about specific biological processes underlying wound healing and catalog potential proteinprotein interactions.

\section{Inferences About Regulatory Mechanisms}

Similar patterns of expression by functionally related genes suggested the hypothesis that clustered genes are regulated by common mechanisms. One approach defines modules of coregulated genes based on the sharing of cis-regulatory motifs (transcription factor binding sites, enhancers, etc.). Livesey and colleagues [2000] documented that genes expressed during the terminal differentiation of photoreceptors share promoter binding sites for the transcription factor CRX. The regulatory relationships of genes and factors within a cluster have been built into motifs and networks, yielding clues about transcriptional networks modulating coordinated expression [Lee et al., 2002; Segal et al., 2005]. Gene expression however is modulated by more than transcription factor activity (for example, heterochromatin remodeling and endogenous gene si- 
lencing). Also, gene expression changes are modulated at the level of translation and posttranslation, as described earlier. Thus, transcriptional networks represent one level of gene expression that can be derived from combining expression clusters with computational analyses of promoters. The next section describes an approach that can define transcriptional regulators as well as post-transcriptional and post-translational regulators of co-regulated genes.

\section{Defining Regulatory Loci That Modulate Gene Expression}

\section{Specificity of Regulation by Chromosome 12 locus}

As a first step toward defining regulatory mechanisms from our retinal injury microarray data, we used online expression genetics to define the genomic location for the upstream modulator(s) controlling wound-healing gene expression [Vazquez et al., 2005]. Our meta-analyses revealed that a locus on chromosome 12 (10-30 Mb locus from the centromere) modulates a group of injury genes that are primarily expressed during the early phases of wound healing (Figure 5.2). The discovery of regulatory networks using meta-analyses of brain expression genetics data raises issues about meta-analysis reliability, wound-healing regulation specificity, and regulation relevance to gene expression in the retina. We addressed the reliability of our observations by testing whether genetic associations are replicated in independent cohorts. For example, chromosome 12 locus is a cis-regulatory locus for Id2, Lpinl, and Sox11 using two independent GeneNetwork databases: UTHSC Brain mRNA U74Av2 (Nov05) and the INIA M430 Brain (April05) database (Figure 4.3). Both databases used independent samples and different array platforms (U74Av2 and M430). We addressed specificity of regulatory loci by comparing regulation across tissues (brain versus hematopoietic stem cells) and functional groups (wound healing versus synaptic genes). From these analyses, we concluded that chromosome 12 locus specifically modulates the expression of wound-healing genes in whole brains. We addressed the relevance of brain data to gene regulation in the retina by examining gene regulation in multiple CNS tissues. 


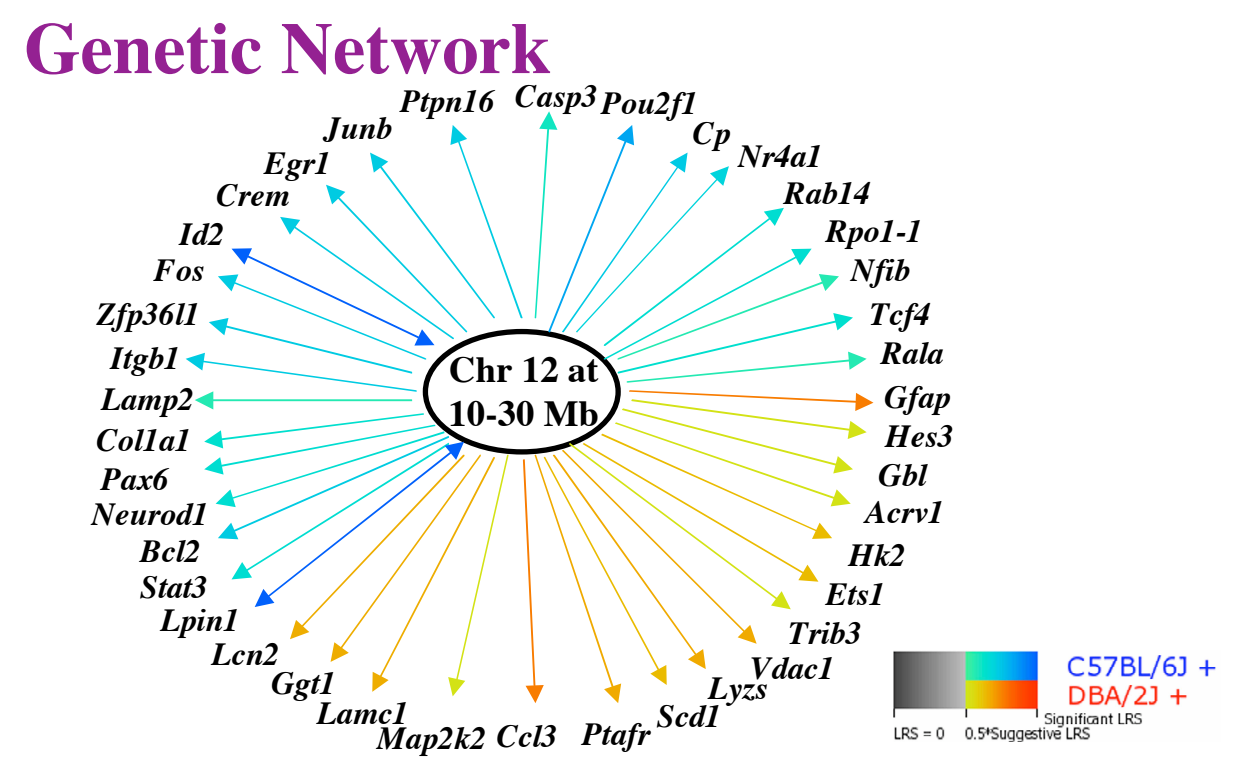

\section{Network's function}

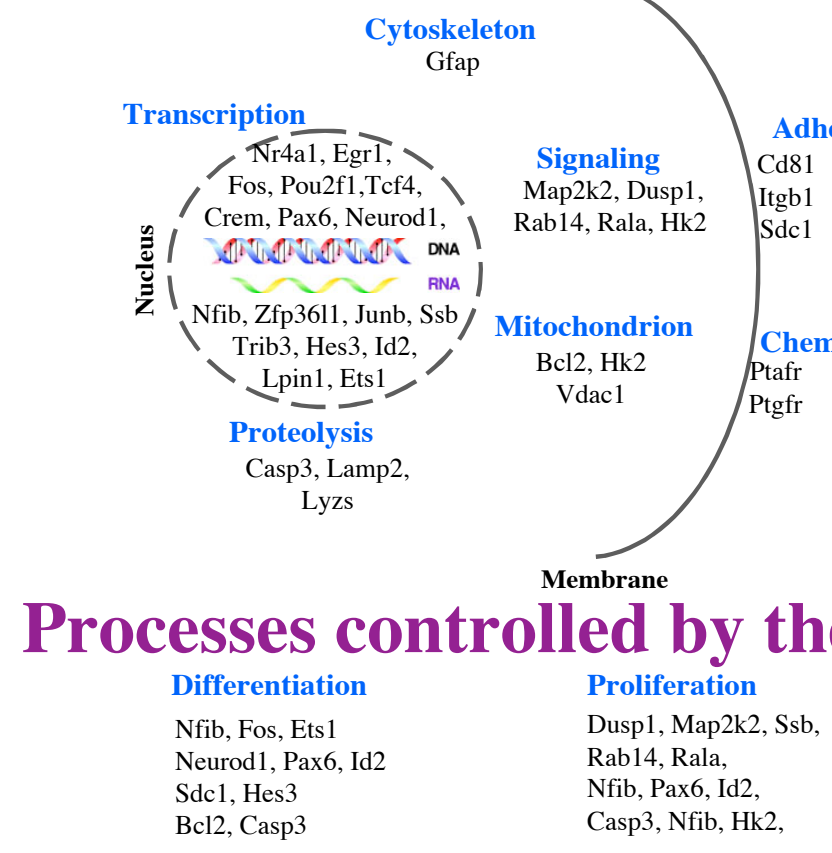

Cell death

Lyzs, Casp3, Bcl2

Nr4a1, Trib3, Id2

Vdac1, Fos

Figure 5.2. Locus on chromosome 12 modulates the expression of genes involved in differentiation, proliferation, and cell death. 
For example, the chromosome 12 locus modulates the expression of Id2, Lpin1, and SoxI1 and in the forebrain, cerebellum, and striatum. The conserved gene regulation across CNS tissues was our motivation for defining regulators for retinal injury genes using available data of genetic interactions from mouse brains. Our RT-PCR data shows that the differential expression of specific transcripts across the retinas of BXD RI strains was in agreement to those displayed in brain. Our analyses of gene expression data injured retina, brain, and spinal cord supports the hypothesis that there is a generalized response to CNS injury [Vazquez et al., 2005]. After injury for example, a group of acute phase genes, mostly transcription factors, are upregulated in injured retina, brain, and spinal cord. Regulation of transcript expression is also known to be maintained across CNS tissues. The transcription factor STAT3 plays an important role in activating cortical astrocytes [Acarin et al., 2001] and retinal Muller glial cells [Wang et al., 2002]. Published neurological phenotypes in BXD RI strains suggest that the chromosome 12 locus can modulate wound healing events across CNS tissues: seizure response of the auditory system, cell death of spiral ganglion cells, and proliferation of adult stem cells in the hippocampus [GeneNetwork IDs 10652, 10655,10434-5, 10437-8, 10504, 10338, 10340-1, and 10378; Willott and Erway, 1998; Neumann and Collins, 1991; Kempermann and Gage, 2002]. These data documents the reliability of our analysis, specificity of chromosome 12 regulation of wound-healing genes, and the relevance of chromosome 12 across CNS tissues including the retina.

We have defined a genetic network that modulates wound-healing gene expression in the CNS. The upstream modulator for this network is located within the 10-30 Mb locus from the centromere of mouse chromosome 12 [Vazquez et al., 2005]. In injured BXD RI strains, the expression of reactive glial genes (Gfap, Fos, Stat3, and Cd81), cell death genes (Casp3), and survival gene (Crygd) correlate with the segregation of C57BL/6 and DBA/2J alleles for the chromosome 12 locus. Reactive glial genes Gfap, Fos, Stat3, and Cd81 and cell death gene Casp3 are expressed at lower levels in strains with the C57BL/6 allele than in strains with the DBA/2J allele; whereas survival gene Crygd is expressed at higher levels in strains with the C57BL/6 allele than in strains with the DBA/2J allele. Published data support the hypothesis that chromosome 12 
network modulates the CNS response to trauma. In BXD RI mouse strains, the DBA2/J allele for the chromosome 12 locus is one of the loci associated with susceptibility to noised-induced cell death of spiral ganglion cells, the cells innervating auditory receptors cells [Willott and Erway, 1998]. The DBA/2J allele also predisposes susceptibility to generalized convulsions when exposed to intense auditory stimulation [Neumann and Collins, 1991]. In contrast, the C57BL/6 allele is one of the loci that confers enhanced neurogenesis and survival of new neurons and astrocytes in adult hippocampus [Kempermann and Gage, 2002]. Moreover, the combined function of genes in the network also suggests that the chromosome12 network can regulate transcription, differentiation, proliferation, and cell death. These processes are characteristic of the early wound-healing response [Vazquez et al., 2004]. Together these data suggest that the chromosome 12 network displays a prominent role in CNS wound healing.

\section{Chromosome 12 Network Modulates Activation of Muller Glia}

Preliminary results suggest that chromosome 12 network modulates transcript changes and phenotypic changes of retinal glial cells either through intrinsic or extrinsic mechanisms. The reactive glial genes Gfap, Fos, Stat3, and Cd81 are expressed at lower levels in strains with the C57BL/6 allele than in strains with the DBA/2J allele. Increased expression and nuclear translocation of transcription factors FOS and STAT3 are characteristic of the early activation of Muller cell signaling cascades [Geller et al., 2001]. Increased expression of cystoskeleton protein GFAP and cell adhesion molecule CD81 are part of a series of phenotypic changes in the Muller cells that include enlargement of cell body, extension of process into areas of retinal damage, and in some cases proliferation [Bignami and Dahl, 1979; Clarke and Geisert, 1998]. Together, these phenotypic changes in glial cells are known as reactive gliosis. The classic marker to measure retinal stress and Muller cell reactivity is GFAP [Lewis and Fisher, 2003]. Immunohistochemistry of GFAP revealed a moderate reactive response by Muller cells in strains with the C57BL/6 allele relative to strains with the $\mathrm{DBA} / 2 \mathrm{~J}$ allele. The chromosome 12 locus also modulates the gene expression of additional reactive gliosis markers, including Cp, Lcn2, Itgbl, and Coll la. 
Several of the chromosome 12 network genes are also differentially expressed in reactive Muller cells including Egr 1, Nr4al, and Lcn2 [GEO data set GSE1979; Gerhardinger et al., 2005]. These data support a model where chromosome 12 locus intrinsically modulates the response of Muller cells to retinal injury by repressing the expression of reactive gliosis genes.

Muller cell reactivity is also extrinsically modulated by the extent of neuronal survival. Experimental models of glaucoma with no direct damage to retina, as in the case of the mechanical crushing of retinal ganglion axons at the optic nerve head, suggest that survival and apoptotic signals are relayed from the ganglion cells to the Muller cells. Indeed, Muller cell reactivity precedes ganglion cell death [Wax and Tezel, 2002]. Injured retina from BXD RI strains display moderate Muller cell reactivity that correlates with lower apoptotic markers (Fos and Casp3) and with higher survival markers (Crygd). Increased expression and activity of transcription factor FOS and protease CASP3 are hallmarks of neuronal cell death in the retina [Hafezi et al., 1999]. Genetic and pharmacological inactivation of FOS and CASP3 protects retinal cells against apoptosis [Wenzel et al., 2000]. Both Fos and Casp3 are controlled by the chromosome 12 network. Additional chromosome 12 network genes involved in the positive regulation of cell death include Nr4a1, Trib3, Vdac1, Lyzs, and Lamp2. Anti-apoptotic genes include Bcl2, Tcf4, and Ptpn16/Duspl. In addition to modulating the expression of apoptosis-related genes, chromosome 12 locus also participates in the regulation of cell death phenotypes in BXD RI mouse strains. The DBA2/J allele is associated with susceptibility to noised-induced cell death of spiral ganglion cells [Willott and Erway, 1998]. Here we showed that the DBA/2J allele correlated with higher levels of apoptotic genes Fos and Casp3 in injured retina. In our studies, the C57BL/6 allele correlated with higher levels of survival gene crystallin gamma $D$. Crystallins are heat-shock like proteins and their upregulation rescues retinal degenerations [Otani et al., 2004]. In injured retina, ganglion cells, photoreceptors, and interneurons upregulate crystallins [Vazquez et al., 2004; Otani et al., 2004]. These data suggest that chromosome 12 can modulate survival and cell death signaling mechanisms of retinal neural cells. The extent of neuronal survival and cell death extrinsically influences the extent of Muller cell reactivity. 


\section{Significance of Chromosome 12 Network in Glaucoma}

The chromosome 12 network represents a novel mechanism that explains differences in the response to retinal injury in murine models. Specifically, our data shows that the DBA/2J allele of the chromosome 12 locus confers susceptibility to neurodegeneration and glial reactivity induced by different experimental models of retinal injury, including toxic injuries and optic nerve crush. Our data and published data [Wax and Tezel, 2002] show that retinal ganglion cells are the most susceptible retinal cells to neurodegeneration after toxic injuries and optic nerve crushes. The work by Simon John's group [2005] has shown that DBA/2J mice are highly susceptible to retinal ganglion cell death due to elevated intraocular pressure, a hallmark of glaucoma. In humans, family history and genetic factors are important in the susceptibility or resistance to glaucoma. Understanding the genetic factors that control neuronal degeneration and glial reactivity in the retinas of BXD RI mice can help elucidate the mechanisms that control susceptibility to glaucoma in humans. The chromosome 12 locus may represent the location for genetic variants responsible for susceptibility to glaucoma. This chromosomal interval has a high degree of homology (that is, synteny) with intervals on the human chromosome 2 (1 to $18 \mathrm{Mb}$ or p25 to p24.3) and on the rat chromosome 6 (34 to $48 \mathrm{Mb}$ or q14 to q16) [NCBI MapViewer, http://www.ncbi.nlm.nih.gov/mapview]. In these syntenic regions, the gene order and gene homology is highly conserved. The next section outlines our work to identify the polymorphic genes responsible for differences in wound-healing response in BXD RI strains.

\section{Finding Regulators of Retinal Wound Healing}

We used gene expression genetics as a guide to identify the location of modulators of woundhealing genes. Regulatory loci can span hundreds of genes (several megabase pairs [Mb]). In our study the regulatory locus spans a region of approximately $20 \mathrm{Mb}$, containing nearly seventy known genes (Figure 5.3). Our approach to define the candidate gene(s) was guided by the biol- 
ogy of the system: co-regulation of wound-healing genes in segregating populations. Therefore, likely candidate modulators are positional, polymorphic genes with expression variability that map to their own locus and with product functions relevant to wound healing. Our data support Id2, Lpin1, and Sox11 as the best current candidate genes.

We searched for genetic variants because that is the variable that accounts for expression phenotypes in the BXD RI strains. Identifying polymorphic genes is assayed by comparing the sequenced genomes of the parental strains. DNA variants within the regulatory region of candidate genes can alter transcription rates by affecting the binding of transcription factors to the promoter. We proposed that the strong self-regulation of Id2 in BXD RI strains is due to a SNP that affects the binding to the CAAT consensus sequence in the promoter (Figure 5.3) [Vazquez et al., 2004]. DNA variants in the transcribed regions also affect hybridization levels of the target to the probes in the array. This is why probes for the same candidate gene but targeting different mRNA regions may provide different hybridization levels. This was illustrated by Aitman and colleagues [1999] who used linkage analysis and microarrays to identify a variant form of $C d 36$ as the fatty acid transporter gene causing insulin resistance in hypertensive rat. The gene in the hypertensive rat has a DNA variant located in the 3' untranslated region that affects its hybridization to the microarray probe derived from the 3' region from wild-type animals. The examples of $I d 2$ and $C d 36$ illustrate that microarray hybridization signals are instructive in suggesting candidate genes. Unfortunately, most commercial microarrays fail to detect DNA variants toward the 5' region due to the 3 ' bias of probes. In these cases, candidate genes can be defined by determining their relevance to wound healing events and determining whether missense mutations alter gene product functions.

A comprehensive and systematic way to determine whether a gene is relevant to wound healing is to examine its expression pattern using the growing collection of gene expression profiling of injured CNS. Transcript information can be complemented and supplemented by mining the literature for a known function in CNS injury and development. One must be careful in 


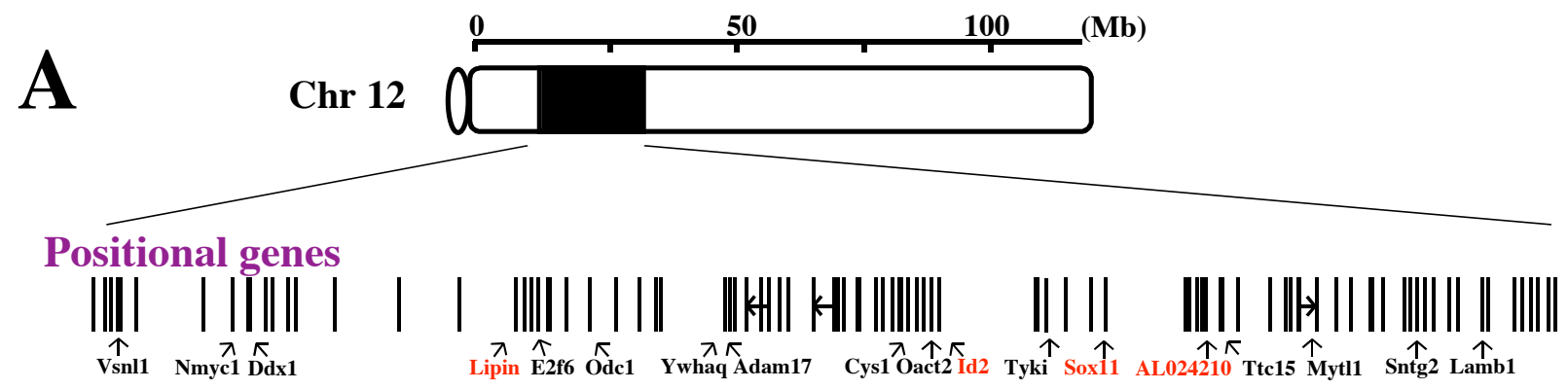

B

Expression variability

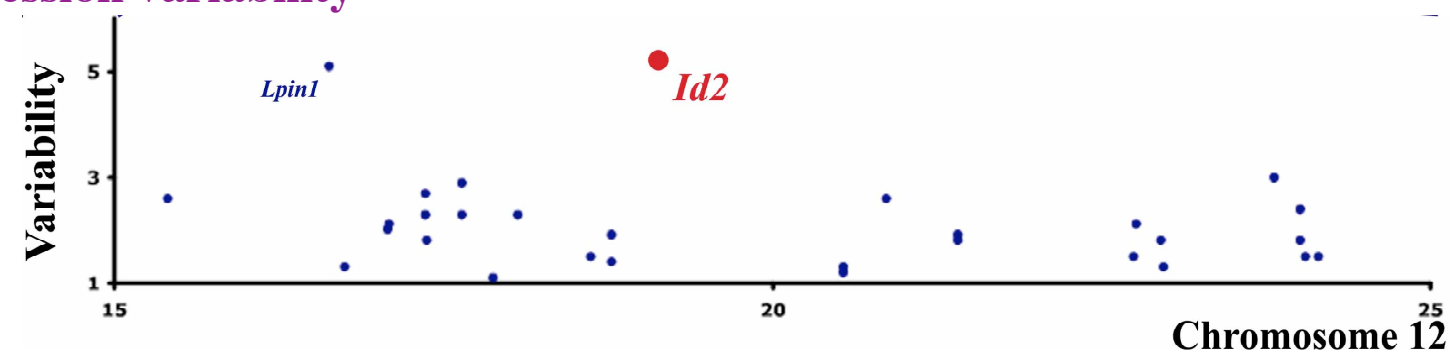

Variability maps to its gene location

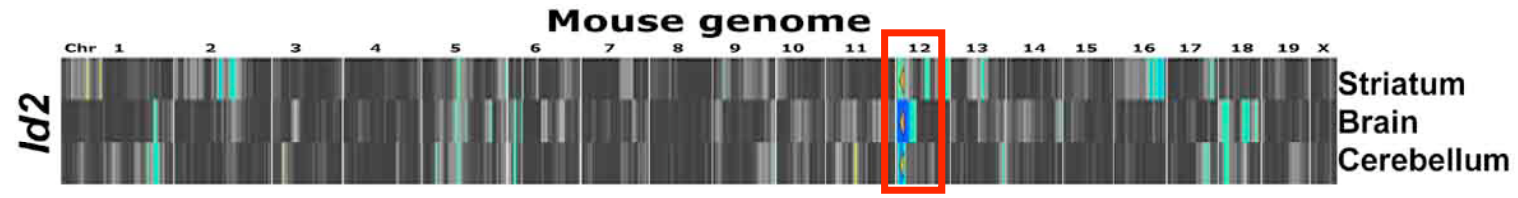

Polymorphic

Relevant SNP

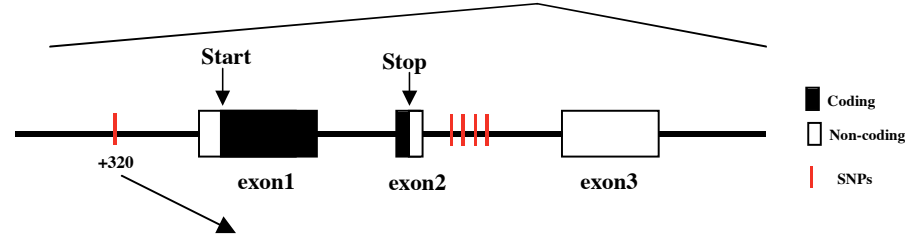

C57BL/ 6 GCCAATG

DBA/2J GCCAATA $\Rightarrow$ LOW Id2 expresser

Network's functional themes:

$\checkmark$ Transcription

$\checkmark$ Neural development

CNS injury:

$\checkmark$ Proliferation

$\checkmark$ Cell death

$\checkmark$ Brain

$\checkmark$ Spinal cord

$\checkmark$ Optic nerve

$\checkmark$ Retina

Figure 5.3. $I d 2$ is a candidate gene modulating the chromosome 12 network. 
ruling out candidate genes based on the absence of information. In our original screen for candidate genes we selected Id2 over Lpin1 based on preponderance of biological data suggesting that the functions of $I d 2$ were consistent with CNS wound healing (Figure 5.3). Post-hoc results with real-time RT-PCR suggest that Lpinl in the retina also displayed high expression differences due to genetic variations and due to trauma (Figure 4.4). Differences in gene product activity in segregating populations, however, can be independent of gene expression differences. A mutation can affect functional domains such as sites for phosphorylation, protein-DNA interaction, and protein-protein interaction that affect the expression of down stream targets without affecting the expression of upstream modulators. Surveys of functional domains are possible by computational analysis of libraries that catalog functional domains based on amino acid sequences. Based on evidence of transcript self regulation, transcript differential expression during retinal development and retinal injury, as well as relevant function of candidates, we have determined that $I d 2$, Lpin1, and Sox11 have strong biological data suggesting their participation in wound healing.

Bioinformatic tools have guided our approach to define hypotheses about modulators of retinal wound healing. This approach is possible with the growing body of biological data that map the global function of cells at different levels (for example, at the level of the genome, transcriptome, proteome, phenome, etc.). High-through put data are, by nature, error prone. Therefore, high-through put data supporting specific hypotheses need to be confirmed by independent means or alternative methods. In this body of work, we illustrate the value of comparative analyses of high-through put data to confirm hypotheses, as we did for our analyses of acute-phase genes in the CNS and regulatory locus analyses [Vazquez et al., 2005]. Skepticism of any data, even published data, is healthy. We initially ignored the use of mice with the Id2-null mutation for studies of adult wound healing because the data by Wang and colleagues [2001] indicated that the $I d 2$-null were post-natal lethal. However, the data by others indicate that $I d 2$-null mice show growth retardation but are viable [Yokota and Mori, 2002]. In perspective, bioinformatic data are reliable when they meet quality control criteria and statistical stringency. In this respect, hypotheses derived from bioinformatic means are no different than those derived from classical means, 
and, should not be required to undergo more stringent, nor less stringent, testing and refinement. Here, we described a method that utilizes a collection of bioinformatic tools to candidate genes responsible for the regulatory locus on chromosome 12 . We also proposed models for the intrinsic and extrinsic modulation of Muller cell reactivity.

\section{Model of Intrinsic Regulation Muller Cell Reactivity}

We integrated computational analyses of biological data (that is, bioinformatics) with the literature to generate potential models of regulation. The known functions of ID2 in the regulation of transcription and neural differentiation are consistent with a model of intrinsic regulation of Muller cell reactivity by the chromosome 12 network. $I d 2$ is expressed by reactive glia in brain, spinal cord, and optic nerve head [Tzeng et al., 1999; Tzeng et al., 2001; Aronica et al., 2001; Hernandez et al., 2002]. Id2 is also upregulated as an immediate early gene in injured retina, brain, and spinal cord [Vazquez et al., 2005]. The retina upregulates Id2 in response to different models of injury including mechanical injury, toxic injury, optic nerve crush, increase intraocular pressure. Expression profiles document that $I d 2$ is highly expressed in reactive Muller cells [GEO data set GSE1979, probe set rc_AI230256_at]. In its classical function, ID2 modulates neural and glial differentiation via the negative regulation of basic helix-loop-helix (bHLH) transcription factors (for example, NeuroD and Olig) [Ross et al., 2003; Liu and Harlan, 2003; Wang et al., 2001]. ID2 binds to bHLH transcription factors to inhibit their binding to E-box sequences, defined by the CANNTG motif [Yokota and Mori, 2002; Ross et al., 2003]. E-boxes are present in promoters of many reactive gliosis markers modulated by chromosome 12 network including Fos [Chaudhary and Skinner, 1999], Stat3 [Shi et al., 1996], Gfap [Tzeng, 2003], Sdc1 [Vihinen et al., 1996], and $C p$ [F. Vazquez, personal in silico promoter observations]. There is evidence that ID2 modulates the gene expression of Fos during Sertoli cell differentiation [Chaudhary and Skinner, 1999]. Based on these data, we proposed a model of an intrinsic program, where ID2 is 
expressed in Muller cells and modulates reactive gliosis markers via E-box mediated transcription (Figure 5.4).

Regulation of Muller cell reactivity by the chromosome 12 network via repression of Ebox mediated transcription is consistent with the known activating signaling pathways of reactive glial markers. Two transcriptional activators of Gfap expression are downstream targets of chro-

mosome 12, Fos and Stat3. Both FOS and STAT3 mediate Gfap expression via activating protein 1 (AP1) binding sites and STAT binding sites (Figure 5.4) [Besnard et al., 1991]. ID2 in turn can mediate control of Fos and Stat3 expression by preventing bHLH from binding to their E-boxes. Thus, ID2 can modulate Gfap expression by negatively regulating Fos and Stat3 expression. Alternatively, ID2 could negatively regulate Gfap expression. Gfap has an E-box in its promoter [Tzeng, 2003]; however, there is no evidence to support that Gfap expression is regulated through the E-box binding site. Repression of E-box mediated transcription of Fos, Stat3, and Gfap, can all contribute to lower levels of Gfap. A similar paradigm might exist for additional chromosome 12 network genes that are expressed in reactive glia. For example, $C p$ has binding sites for FOS and STAT3 as well as E-box binding sites [F. Vazquez, personal in silico promoter observations]. In this model, chromosome12 network modulation of Muller cell reactivity offers a new level of regulation via ID2 that is consistent with current activating mechanisms of retinal glial cells.

\section{Perspectives on Testing Candidate Genes}

\section{Cellular Localization}

Whole genome-wide analyses are powerful approaches to defining regulatory mechanisms. The next level of analyses requires the experimental confirmation of these regulatory mechanisms. A first step toward evidence of causation and mechanism of action is determining cellular localization of candidate modulators. When antibodies are available, immunohistochemistry is an efficient, and cost effective technique to determine the cellular source of gene products. We 


\section{Intrinsic:}
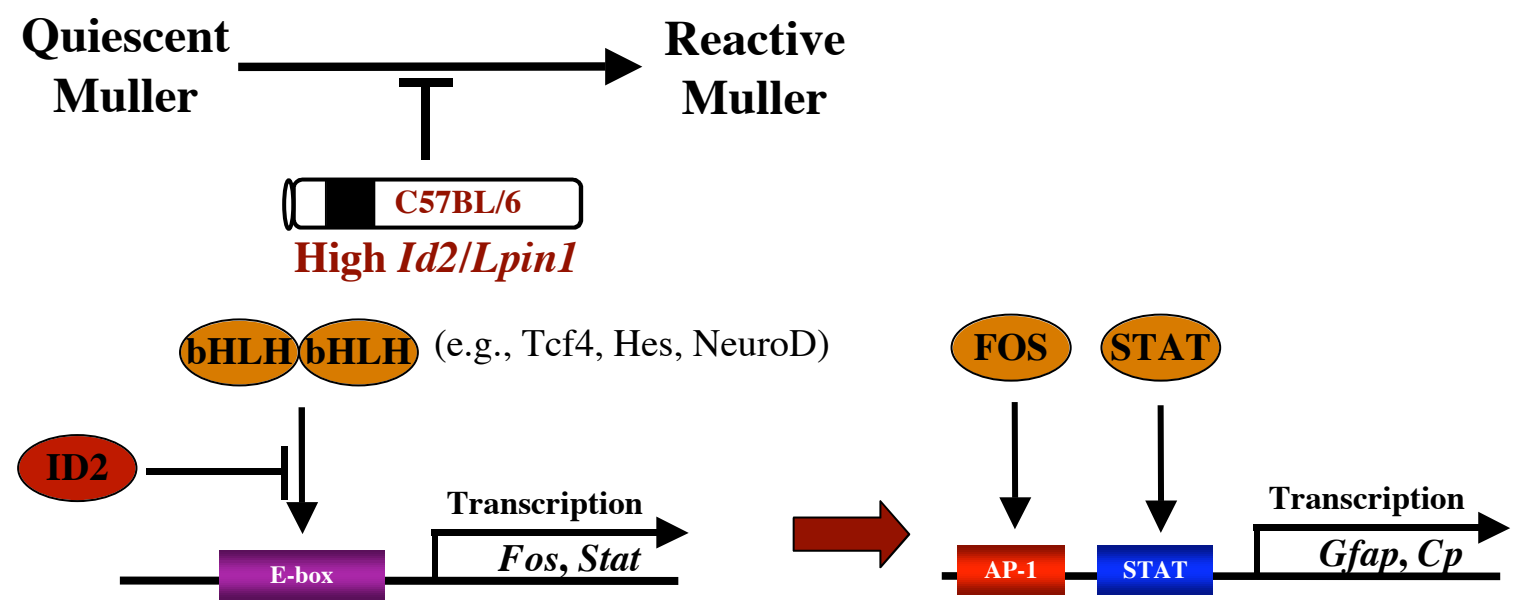

\section{Extrinsic:}

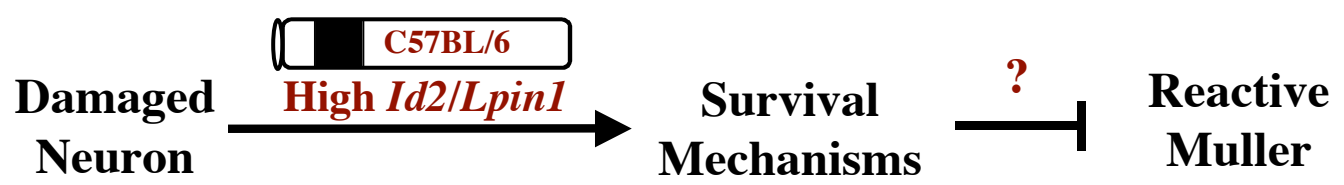

Figure 5.4. Model of Muller cell activation by chromosome 12 network. 
however found that antibodies need appropriate controls to confidently determine the cellular source. For example, we found that an ID2 polyclonal antibody (Santa Cruz Biotechnology, SC489) labels interneurons of the retina. However, when we tested against retina from animals with a null-mutation for $I d 2$ we found virtually no difference between wild-type and knock-out animals (Appendix D, Figure D.1). The non-specificity of the ID2 antibody was surprising as the antibody in western blots recognizes an antigen that corresponds to the molecular weight of ID2, and the intensity of immunoreaction is proportional to transcript levels (Figure 4.4). LPIN1 antibody (Alpha Diagnostic, LPN11-A) is also non-specific in tissue. In both cases, antibodies were generated against small, synthetic peptides that may not reflect the protein conformation in tissues. The limited availability and reliability of antibodies to assess gene function illustrates the current limitation of protein and molecular methods to keep up with gene-discovery approaches.

\section{Gene Dosage Effect Using BXD RI Strains}

We tested the role of candidate genes during wound healing by testing the effect of gene expression dosage in BXD RI mouse strains. The natural range of gene expression in BXD RI mouse strains offers a powerful tool to test and refine hypotheses. Candidate genes whose expression dosage correlate with the degree of resistance or susceptibility of retinal wound healing provide strong evidence of wound healing modulation. In this project, we showed that high levels of expression for Id 2 and Lpinl correlate with lower expression levels for markers of acute phase, reactive gliosis, and cell death. Our results nicely complement published neurological studies which showed that the chromosomal region around the Id2 and Lpin1 loci correlate with the extent of audiogenic seizure, neuronal hearing cell death, and hippocampal adult neurogenesis [Willott and Erway, 1998; Neumann and Collins, 1991; Kempermann and Gage, 2002]. BXD RI strains also offer a system to differentiate the contribution of candidate genes to wound healing by increasing the resolution of the regulatory locus, currently at 10-30 Mb from centromere. The new BXD RI strain set developed by Peirce and colleagues [2004] offer two chromosomal recombinations between genetic markers at 11 and $22 \mathrm{Mb}$ (D12Mit182 and D12Mit84) and be- 
tween genetic markers at 22 and $30 \mathrm{Mb}$ (D12Mit84 and D12Mit153). Additional strains

(BXDA24, BXDA35, BXDA7) could help refine the correlations between candidate genes Lpin1, Id2, Sox11, and AL024210 (located at 15.9, 21.6, 23.9, and 25.2 Mb) and extent of retinal wound healing. There is an important experimental design consideration: Since each BXD RI mouse strain is a unique recombination of the parental genomes, the experiment needs to include sufficient number of strains to significantly correlate gene dosage level effects on wound healing phenotype with the segregation of corresponding alleles. At a minimum, the parental strains, the F1 cross, and an additional strain from each end of the expression range need to be included in the experiment. Our data support that BXD RI strains are valuable in increasing the resolution of linkage mapping and correlating gene dosage with susceptibility or resistance to retinal injury.

\section{In Vitro Testing}

Cell culture systems can also provide evidence of modulation. Our results show that RNA interference in cultured cells is a practical way to decrease transcript levels. Short interfering oligonucleotides that target $I d 2$ and $C d 81$ mRNAs efficiently decreased $I d 2$ and $C d 81$ transcript levels in cultured astrocytes. The RNA interference culture system also facilitates the testing of specific hypotheses. Decreases in the levels of $I d 2$ and $C d 81$ lead to a decrease in wound-healing rates of cultured astrocytes. We also determined that $I d 2$ silencing leads to a decrease in wound-healing rates by altering proliferation of cultured astrocytes. In contrast, the silencing of the cell membrane gene $C d 81$ leads to a decrease of wound healing by altering the migration of cultured astrocytes [X. Wang and E. Geisert, personal observations]. Phenotypes from gene silencing experiments often resembled phenotypes from cells obtained from mice with the null-mutation. Astrocytes from the Cd81-/- mice also display decreased wound-healing rates [X. Wang and E. Geisert, personal observations]. Likewise, our observation that lower $I d 2$ levels decrease proliferation rates is consistent with data from neuronal precursor cells from the Id2-/- mice [Wang et al., 2001]. These observations support the reliability of RNA interference to define the function of a candidate gene in a specific cell type. The reductionist approach of RNA interference in cell cul- 
ture is valuable in identifying downstream and upstream targets [Berns et al., 2004]. Since ID2 can regulate proliferation and differentiation by regulating transcription, future experiments will define the transcripts that are affected by Id2 silencing. As with any gain- or loss-of-function experiments, the observations of cause and effect can be strengthen if the altered phenotype can be reversed either by genetic means or pharmacological means. In the case of RNA interference, the phenotype can be reversed by ectopic expression of the target gene. When testing of hypothesis in cell culture, one must keep in mind that while cell culture systems offer direct cellular accessibility and microenvironmental control, as well as efficient comparison between many experimental conditions, they are in artificial environments that differ from the normal, complex tissue environment. Nonetheless, in vitro systems are a reliable first line of testing that provides a feasible and cost-effective way to screen a list of candidate targets [Leveillard et al., 2004]. Moreover, cell culture systems are also amenable to manipulation to determine the mechanisms of action.

\section{In Vivo Testing}

Powerful approaches to test candidate genes in animal models include the deletion or over expression of candidate genes, as well as, the rescue or introduction of candidate mutations. Reverse genetic approaches provide a powerful way to assess gene function, but interpretations from transgenic models can be complicated by unintended complementation during development, genetic background, and epigenetic influences. One clear example is the $R b$ gene. In humans a mutation in this gene causes retinoblastoma. However in the mouse the $R b$-null mutation fails to produce retinoblastoma due to compensation by the other members of the RB family (p107 or p130) [Dyer and Bremner, 2005]. Another concern with transgenic mice is the genetic background and flanking genes of the animals used for transgenics are important factors that can affect the interpretation of the results [Wolfer et al., 2002]. Geisert and colleagues [2002] documented that when the null mutation of $C d 81$ is placed on different genetic backgrounds, there is a remarkable range in the penetrance of the null allele phenotype, demonstrating that the mutation can be affected by modifier loci. Reverse genetic approaches to determine the gene function dur- 
ing wound healing also need to consider that strains differ in their response to CNS injury. For example, there are strains that display resistance to neurotoxic injury (C57BL/6, BALB/cJ, 129S1/SvImJ, C3H, ICR, SJL) and strains that are susceptible to neurotoxicity (DBA/2J, CD-1, FVB/N, 129T2 Sv/EMS, and C57BL/10) [Inman et al., 2002]. Furthermore, functional consequences of changes in gene activity are often asymmetric, meaning that the over expression and the deletion of a gene may not yield opposite phenotypes. For example, the transgenic mice overexpressing the antioxidant enzyme glutathione peroxidase 1 display increased tolerance to hyperoxia, whereas the mice lacking glutathione peroxidase 1 display no effects on animal survival under hyperoxia [Ho et al., 1998]. These studies suggest the complexity of gene expression regulation by genetic interactions and by mechanisms independent of nucleotide sequence (epigenetics).

\section{Suggested Protocol}

Based on our successes and failures we proposed the following protocol for determining and testing candidate genes: (1) confirm transcript differential due to genetic background and injury; (2) improve the power and resolution of genetic mapping by using new BXD RI strain set developed by Pierce and colleagues [2004]; (3) determine cellular co-localization of candidate gene using in situ hybridization or immunohistochemistry, (4) examine the effect of gene silencing and overexpression in the appropriate cell system and the appropriate functional assay; (5) determine the candidate gene effect on wound healing using transgenics such as mice with gene knock-in and null-mutations; and (6) if transgenics are not available or viable, the natural gene dosage of the BXD RI strains can be used to supplement (and complement) the testing of candidate genes. Of particular interest would be to compare perturbed transcriptional networks and wound-healing response in segregating populations with those in single-gene perturbation experiments. This method can address the weaknesses of forward and reverse genetic approaches [Mehrabian et al., 2005]. Specifically, the segregating population (for example, the BXD RI strain set) is used to define genetic networks associated with the complex regulation of wound healing. Single-gene 
perturbation experiments (for example, the Id2 or Lpin1 null mice) are used to define the effects on specific transcriptional networks. The altered gene expression signature and wound-healing response in transgenics is mapped back into the segregating population and interpreted in the context where the complex trait had initially been observed. Additionally, cell culture systems can help determine the direction of flow and the gene product interactions within the network. Therefore, I propose that observations from different lines of experimental evidence are not mutually exclusive but together can add to our understanding of gene expression regulation and their relationship to the progression of wound healing.

\section{Conclusion}

The present series of studies defined global changes that occur after injury. They also defined a genetic network that modulates the retinal wound healing response. Our data supports that the chromosome 12 network intrinsically or extrinsically modulates early Muller cell activation. The upstream regulator for this network is located on a locus of chromosome 12. Id2 and Lpin1 are current candidate modulators for the network as higher levels of Id2 and Lpin I correlated with higher levels of the survival genes, and with lower levels of reactive gliosis genes and apoptotic genes. In this body of work, I have moved beyond cataloging changes in the transcriptome to identifying candidate genes modulating the retinal response to injury. During this process I developed an integrated approach of gene expression profiling and higher-level bioinformatic analyses to define genetic networks. This work not only advances our understanding of the molecular networks controlling the CNS response to injury, but may also form the basis for interventions that can rescue injured neurons and re-establish lost CNS connections. 


\section{List of References}


Abiola O, Angel JM, Avner P, et al. The nature and identification of quantitative trait loci: a community's view. Nat Rev Genet. 2003;4:911-6.

Acarin L, Gonzalez B, Castellano B. Glial activation in the immature rat brain: implication of inflammatory transcription factors and cytokine expression. Prog Brain Res. 2001:375-89.

Ahmed F, Brown KM, Stephan DA, Morrison JC, Johnson EC, Tomarev SI. Microarray analysis of changes in mRNA levels in the rat retina after experimental elevation of intraocular pressure. Invest Ophthalmol Vis Sci. 2004; 45:1247-58.

Aitman TJ, Glazier AM, Wallace CA, et al. Identification of Cd36 (Fat) as an insulin-resistance gene causing defective fatty acid and glucose metabolism in hypertensive rats. Nat Genet. 1999;21:76-83.

Alge CS, Priglinger SG, Neubauer AS, et al. Retinal pigment epithelium is protected against apoptosis by alphaB-crystallin. Invest Ophthalmol Vis Sci. 2002;43:3575-82.

Anderson DH, Ozaki S, Nealon M, et al. Local cellular sources of apolipoprotein E in the human retina and retinal pigmented epithelium: implications for the process of drusen formation. Am J Ophthalmol. 2001;131:767-81.

Aronica E, Vandeputte DA, van Vliet EA, Lopes da Silva FH, Troost D, Gorter JA. Expression of Id proteins increases in astrocytes in the hippocampus of epileptic rats. Neuroreport. 2001;12:2461-5.

Atanasoski S, Scherer SS, Nave KA, Suter U. Proliferation of Schwann cells and regulation of cyclin D1 expression in an animal model of Charcot-Marie-Tooth disease type 1A. J Neurosci Res. 2002;67:443-9.

Behar-Cohen FF, Thillaye-Goldenberg B, de Bizemont T, et al. EIU in the rat promotes the potential of syngeneic retinal cells injected into the vitreous cavity to induce PVR. Invest Ophthalmol Vis Sci. 2000;41:3915-24.

Berge KE, Tian H, Graf GA, et al. Accumulation of dietary cholesterol in sitosterolemia caused by mutations in adjacent ABC transporters. Science. 2000;290:1771-5.

Berns K, Hijmans EM, Mullenders J, et al. A large-scale RNAi screen in human cells identifies new components of the p53 pathway. Nature. 2004;428:431-7.

Besnard F, Brenner M, Nakatani Y, Chao R, Purohit HJ, Freese E. Multiple interacting sites regulate astrocyte-specific transcription of the human gene for glial fibrillary acidic protein. $\mathrm{J}$ Biol Chem. 1991;266:18877-83.

Beyer A, Hollunder J, Nasheuer HP, Wilhelm T. Post-transcriptional expression regulation in the yeast Saccharomyces cerevisiae on a genomic scale. Mol Cell Proteomics. 2004;3:1083-92.

Bignami A, Dahl D. The radial glia of Müller in the rat retina and their response to injury. An immunofluorescence study with antibodies to the glial fibrillary acidic (GFA) protein. Exp Eye Res. 1979;28:63-9.

Blackshaw S, Harpavat S, Trimarchi J, et a; Genomic analysis of mouse retinal development. PLoS Biol. 2004;2:E247.

Boucheix C, Rubinstein E. Tetraspanins. Cell Mol Life Sci. 2001;58:1189-205.

Bringmann A, Reichenbach A. Role of Müller cells in retinal degenerations. Front Biosci. 2001;6:E72-92.

Broman KW. Mapping expression in randomized rodent genomes. Nat Genet. 2005; 37:209-10.

Burke JM, Smith JM. Retinal proliferation in response to vitreous hemoglobin or iron. Invest Ophthalmol Vis Sci. 1981;20:582-92.

Butte A. The use and analysis of microarray data. Nat Rev Drug Discov. 2002;1:951-60.

Bystrykh L, Weersing E, Dontje B, Sutton S, Pletcher MT, Wiltshire T, Su AI, Vellenga E, Wang J, Manly KF, Lu L, Chesler EJ, Alberts R, Jansen RC, Williams RW, Cooke MP, de Haan G. 
Uncovering regulatory pathways that affect hematopoietic stem cell function using 'genetical genomics'. Nat Genet. 2005; 37:225-32.

Campochiaro PA, Bryan JA 3rd, Conway BP, Jaccoma EH. Intravitreal chemotactic and mitogenic activity. Implication of blood-retinal barrier breakdown. Arch Ophthalmol. 1986;104:1685-7.

Cao W, Wen R, Li F, Cheng T, Steinberg RH. Induction of basic fibroblast growth factor mRNA by basic fibroblast growth factor in Muller cells. Invest Ophthalmol Vis Sci. 1997;38:1358-66.

Chaudhary J, Skinner MK. Basic helix-loop-helix proteins can act at the E-box within the serum response element of the c-fos promoter to influence hormone-induced promoter activation in Sertoli cells. Mol Endocrinol. 1999;13:774-86.

Chaum E. Proliferative vitreoretinopathy. Int Ophthalmol Clin. 1995;35:163-73.

Chen H, Sharp BM. Content-rich biological network constructed by mining PubMed abstracts. BMC Bioinformatics. 2004;5:147.

Chen L, Dentchev T, Wong R, Hahn P, Wen R, Bennett J, Dunaief JL. Increased expression of ceruloplasmin in the retina following photic injury. Mol Vis. 2003;30;9:151-8.

Chen L, Wu W, Dentchev T, et al. Light damage induced changes in mouse retinal gene expression. Exp Eye Res. 2004;79:239-47.

Chesler EJ, Lu L, Wang J, Williams RW, Manly KF. WebQTL: rapid exploratory analysis of gene expression and genetic networks for brain and behavior. Nat Neurosci. 2004;7:485-6.

Chesler EJ, Lu L, Shou S, et al. Complex trait analysis of gene expression uncovers polygenic and pleiotropic networks that modulate nervous system function. Nat Genet. 2005;37:233-42.

Cheung VG, Conlin LK, Weber TM, et al. Natural variation in human gene expression assessed in lymphoblastoid cells. Nat Genet. 2003;33:422-5.

Cho EY, Choi HL, Chan FL. Expression pattern of glycoconjugates in rat retina as analyzed by lectin histochemistry. Histochemical J. 2002;34:589-600.

Chu Y, Humphrey MF, Alder VV, Constable IJ. Immunocytochemical localization of basic fibroblast growth factor and glial fibrillary acidic protein after laser photocoagulation in the Royal College of Surgeons rat. Aust N Z J Ophthalmol. 1998;26:87-96.

Clarke K, Geisert EE Jr. The target of the antiproliferative antibody (TAPA) in the normal and injured rat retina. Mol Vision. 1998;4:3.

Darvasi A. Genomics: Gene expression meets genetics. Nature. 2003;422:269-70.

Dash PK, Kobori N, Moore AN. A molecular description of brain trauma pathophysiology using microarray technology: an overview. Neurochem Res. 2004;29:1275-86.

Deng Y, Yao L, Chau L, et al. N-Myc downstream-regulated gene 2 (NDRG2) inhibits glioblastoma cell proliferation. Int J Cancer. 2003;106:342-7.

Di Giovanni S, Knoblach SM, Brandoli C, Aden SA, Hoffman EP, Faden AI. Gene profiling in spinal cord injury shows role of cell cycle in neuronal death. Ann Neurol. 2003;53:454-68.

Diaz E, Ge Y, Yang YH, et al. Molecular analysis of gene expression in the developing pontocerebellar projection system. Neuron. 2002;36:417-34.

Dijkstra S, Geisert EE Jr, Gispen WH, Bar PR, Joosten EA. Up-regulation of CD81 (target of the antiproliferative antibody; TAPA) by reactive microglia and astrocytes after spinal cord injury in the rat. J Comp Neurol. 2000;428:266-77.

Dorrell MI, Aguilar E, Weber C, Friedlander M. Global gene expression analysis of the developing postnatal mouse retina. Invest Ophthalmol Vis Sci. 2004;45:1009-19.

Duke DC, Moran LB, Turkheimer FE, Banati R, Graeber MB. Microglia in culture: what genes do they express? Dev Neurosci. 2004; 26:30-7. 
Dyer MA, Cepko CL. Control of Muller glial cell proliferation and activation following retinal injury. Nat Neurosci. 2000;3:873-80.

Dyer MA, Bremner R. The search for the retinoblastoma cell of origin. Nat Rev Cancer. 2005;5:91-101.

Evans SJ, Datson NA, Kabbaj M, et al. Evaluation of Affymetrix Gene Chip sensitivity in rat hippocampal tissue using SAGE analysis. Serial Analysis of Gene Expression. Eur J Neurosci. 2002;16:409-13.

Fischer AJ, Reh TA. Potential of Muller glia to become neurogenic retinal progenitor cells. Glia. 2003;43:70-6.

Fisher SK, Stone J, Rex TS, Linberg KA, Lewis GP. Experimental retinal detachment: a paradigm for understanding the effects of induced photoreceptor degeneration. Prog Brain Res. 2001;131:679-98.

Fisher SK, Lewis GP. Muller cell and neuronal remodeling in retinal detachment and reattachment and their potential consequences for visual recovery: a review and reconsideration of recent data. Vision Res. 2003;43:887-97.

Fisher SK, Lewis GP, Linberg KA, Verardo MR. Cellular remodeling in mammalian retina: results from studies of experimental retinal detachment. Prog Retin Eye Res. 2005;24:395-431.

Fraser AG, Marcotte EM. A probabilistic view of gene function. Nat Genet. 2004;36:559-64.

Geisert EE Jr, Yang L, Irwin MH. Astrocyte growth, reactivity, and the target of the antiproliferative antibody, TAPA. J Neurosci. 1996;16:5478-87.

Geller SF, Lewis GP, Fisher SK. FGFR1, signaling, and AP-1 expression after retinal detachment: reactive Muller and RPE cells. Invest Ophthalmol Vis Sci. 2001;42:1363-9.

Gerhardinger C, Costa MB, Coulombe MC, Toth I, Hoehn T, Grosu P. Expression of acute-phase response proteins in retinal Muller cells in diabetes. Invest Ophthalmol Vis Sci. 2005;46:34957.

Gilbert RE, Cox AJ, Kelly DJ, et al. Localization of secreted protein acidic and rich in cysteine (SPARC) expression in the rat eye. Connect Tissue Res. 1999;40:295-303.

Gleichmann M, Buchheim G, El-Bizri H, et al. Identification of inhibitor-of-differentiation 2 (Id2) as a modulator of neuronal apoptosis. J Neurochem. 2002; 80:755-62.

Gregor Z, Ryan SJ. Combined posterior contusion and penetrating injury in the pig eye. II. Histological features. Br J Ophthalmo. 1982;66:799-804.

Grierson I, Hiscott P, Hogg P, Robey H, Mazure A, Larkin G. Development, repair and regeneration of the retinal pigment epithelium. Eye. 1994;8:255-62.

Grimm C, Wenzel A, Hafezi F, Reme CE. Gene expression in the mouse retina: the effect of damaging light. Mol Vis. 2000;6:252-60.

Hack MA, Sugimori M, Lundberg C, Nakafuku M, Gotz M. Regionalization and fate specification in neurospheres: the role of Olig2 and Pax6. Mol Cell Neurosci. 2004;25:664-78.

Hageman GS, Anderson DH, Johnson LV, et al. A common haplotype in the complement regulatory gene factor $\mathrm{H}(\mathrm{HF} 1 / \mathrm{CFH})$ predisposes individuals to age-related macular degeneration. Proc Natl Acad Sci U S A. 2005;102:7227-32.

Hemler ME. Specific tetraspanin functions. J Cell Bio. 2001;55:1103-7.

Hernandez MR, Agapova OA, Yang P, Salvador-Silva M, Ricard CS, Aoi S. Differential gene expression in astrocytes from human normal and glaucomatous optic nerve head analyzed by cDNA microarray. Glia. 2002; 38:45-64.

Heyer LJ, Kruglyak S, Yooseph S. Exploring expression data: identification and analysis of coexpressed genes. Genome Res. 1999;9:1106-15. 
Horvath CM. STAT proteins and transcriptional responses to extracellular signals. Trends Biochem Sci. 2000;25:496-502.

Horwitz J. Alpha-crystallin. Exp Eye Res. 2003;76:145-53.

Huard JM, Forster CC, Carter ML, Sicinski P, Ross ME. Cerebellar histogenesis is disturbed in mice lacking cyclin D2. Development. 1999; 126:1927-35.

Hubner N, Wallace CA, Zimdahl H, et al. Integrated transcriptional profiling and linkage analysis for identification of genes underlying disease. Nat Genet. 2005;37:243-53.

Humphrey MF, Constable IJ, Chu Y, Wiffen S. A quantitative study of the lateral spread of Muller cell responses to retinal lesions in the rabbit. J Comp Neurol. 1993;334:545-58.

Inman D, Guth L, Steward O. Genetic influences on secondary degeneration and wound healing following spinal cord injury in various strains of mice. J Comp Neurol. 2002;451:225-35.

Irizarry RA, Bolstad BM, Collin F, Cope LM, Hobbs B, Speed TP. Summaries of Affymetrix GeneChip probe level data. Nucleic Acids Res. 2003;31:e15.

Izumi Y, Shimamoto K, Benz AM, Hammerman SB, Olney JW, Zorumski CF. Glutamate transporters and retinal excitotoxicity. Glia. 2002;39:58-68.

John SW. Mechanistic insights into glaucoma provided by experimental genetics the cogan lecture. Invest Ophthalmol Vis Sci. 2005;46:2649-61.

Johnson JM, Castle J, Garrett-Engele P, et al. Genome-wide survey of human alternative premRNA splicing with exon junction microarrays. Science 302;2003:2141-2144.

Jones BW, Watt CB, Frederick JM, et al. Retinal remodeling triggered by photoreceptor degenerations. J Comp Neurol. 2003;464:1-16.

Jones BW. Transcriptional control of glial cell development in Drosophila. Dev Biol. 2005;278:265-73.

Karp CL, Grupe A, Schadt E, et al. Identification of complement factor 5 as a susceptibility locus for experimental allergic asthma. Nat Immunol. 2000;1:221-6.

Kempermann G, Gage FH. Genetic influence on phenotypic differentiation in adult hippocampal neurogenesis. Brain Res Dev Brain Res. 2002;134:1-12.

Kim IK, Arroyo JG. Mechanisms in proliferative vitreoretinopathy. Ophthalmol Clin North Am. 2002;15:81-6.

Kinouchi R, Takeda M, Yang L, et al. Robust neural integration from retinal transplants in mice deficient in GFAP and vimentin. Nat Neurosci. 2003;6:863-8.

Klein JA, Ackerman SL. Oxidative stress, cell cycle, and neurodegeneration. J Clin Invest. 2003; 111:785-93.

Knuppel R, Dietze P, Lehnberg W, Frech K, Wingender E. TRANSFAC retrieval program: a network model database of eukaryotic transcription regulating sequences and proteins. J Comput Biol. 1994;1:191-8.

Kraft AD, Johnson DA, Johnson JA. Nuclear factor E2-related factor 2-dependent antioxidant response element activation by tert-butylhydroquinone and sulforaphane occurring preferentially in astrocytes conditions neurons against oxidative insult. J Neurosci. 2004;24:1101-12.

LaVail MM, Unoki K, Yasumura D, Matthes MT, Yancopoulos GD, Steinberg RH. Multiple growth factors, cytokines, and neurotrophins rescue photoreceptors from the damaging effects of constant light. Proc Natl Acad Sci U S A. 1992;89:11249-53.

Lee TI, Rinaldi NJ, Robert F, et al. Transcriptional regulatory networks in Saccharomyces cerevisiae. Science. 2002;298:799-804.

Leveillard T, Mohand-Said S, Lorentz O, et a. Identification and characterization of rod-derived cone viability factor. Nat Genet. 2004;36:755-9. 
Lewis GP, Fisher SK. Up-regulation of glial fibrillary acidic protein in response to retinal injury: its potential role in glial remodeling and a comparison to vimentin expression. Int Rev Cytol. 2003;230:263-90.

Liang FQ, Godley BF. Oxidative stress-induced mitochondrial DNA damage in human retinal pigment epithelial cells: a possible mechanism for RPE aging and age-related macular degeneration. Exp Eye Res. 2003;76:397-403.

Lin Z, Crockett DK, Jenson SD, Lim MS, Elenitoba-Johnson KS. Quantitative proteomic and transcriptional analysis of the response to the p38 mitogen-activated protein kinase inhibitor SB203580 in transformed follicular lymphoma cells. Mol Cell Proteomics. 2004;3:820-33.

Liou GI, Pakalnis VA, Matragoon S, et al. HGF regulation of RPE proliferation in an IL1B/retinal hole-induced rabbit model of PVR. Mol Vis. 2002;8:494-501.

Lipshutz RJ, Fodor SP, Gingeras TR, Lockhart DJ. High density synthetic oligonucleotide arrays. Nat Genet. 1999;21:20-4.

Liu C, Peng M, Laties AM, Wen R. Preconditioning with bright light evokes a protective response against light damage in the rat retina. J Neurosci. 1998;18:1337-44.

Liu KJ, Harland RM. Cloning and characterization of Xenopus Id4 reveals differing roles for Id genes. Dev Biol. 2003;264:339-51.

Livesey FJ, Furukawa T, Steffen MA, Church GM, Cepko CL. Microarray analysis of the transcriptional network controlled by the photoreceptor homeobox gene Crx. Curr Biol. 2000;10:301-10.

Lockhart DJ, Dong H, Byrne MC, et al. Expression monitoring by hybridization to high-density oligonucleotide arrays. Nat Biotechnol. 1996;14:1675-80.

MacLaren RE. Development and role of retinal glia in regeneration of ganglion cells following injury. Br J Ophthalmol. 1996;80:458-64.

Manly KF, Wang J, Williams RW. Weighting by heritability for detection of quantitative trait loci with microarray estimates of gene expression. Genome Biology. 2005;6R27.

Marc RE, Jones BW, Watt CB, Strettoi E. Neural remodeling in retinal degeneration. Prog Retin Eye Res. 2003;22:607-55.

Marsich E, Vetere A, Di Piazza M, Tell G, Paoletti S. The PAX6 gene is activated by the basic helix-loop-helix transcription factor NeuroD/BETA2. Biochem J. 2003;376:707-15.

Mata J, Marguerat S, Bahler J. Post-transcriptional control of gene expression: a genome-wide perspective. Trends Biochem Sci. 2005;30:506-14.

Matzilevich DA, Rall JM, Moore AN, Grill RJ, Dash PK. High-density microarray analysis of hippocampal gene expression following experimental brain injury. J Neurosci Res. 2002;67:646-63.

Mervin K, Valter K, Maslim J, Lewis G, Fisher S, Stone J. Limiting photoreceptor death and deconstruction during experimental retinal detachment: the value of oxygen supplementation. Am J Ophthalmol. 1999;128:155-64.

Mijalski T, Harder A, Halder T, et al. Identification of coexpressed gene clusters in a comparative analysis of transcriptome and proteome in mouse tissues. Proc Natl Acad Sci U S A. 2005;102:8621-6.

Miller NM, Oberdorfer M. Neuronal and neuroglial responses following retinal lesions in the neonatal rats. J Comp Neurol. 1981;202:493-504.

Mitchelmore C, Buchmann-Moller S, Rask L, West MJ, Troncoso JC, Jensen NA. NDRG2: a novel Alzheimer's disease associated protein. Neurobiol Dis. 2004;16:48-58.

Moran LB, Duke DC, Turkheimer FE, Banati RB, Graeber MB. Towards a transcriptome definition of microglial cells. Neurogenetics. 2004; 5:95-108. 
Morley M, Molony CM, Weber TM, et al. Genetic analysis of genome-wide variation in human gene expression. Nature. 2004;430:743-7.

Natale JE, Ahmed F, Cernak I, Stoica B, Faden AI. Gene expression profile changes are commonly modulated across models and species after traumatic brain injury. J Neurotrauma. 2003;20:907-27.

Neumann PE, Collins RL. Genetic dissection of susceptibility to audiogenic seizures in inbred mice. Proc Natl Acad Sci U S A. 1991;88:5408-12.

Nichols NR. Ndrg2, a novel gene regulated by adrenal steroids and antidepressants, is highly expressed in astrocytes. Ann N Y Acad Sci. 2003; 1007:349-56.

Novatchkova M, Eisenhaber F. Can molecular mechanisms of biological processes be extracted from expression profiles? Case study: endothelial contribution to tumor-induced angiogenesis. Bioessays. 2001 Dec;23:1159-75.

Otani A, Dorrell MI, Kinder K, et al. Rescue of retinal degeneration by intravitreally injected adult bone marrow-derived lineage-negative hematopoietic stem cells. J Clin Invest. 2004;114:765-74.

Pagliuca A, Gallo P, De Luca P, Lania L. Class A helix-loop-helix proteins are positive regulators of several cyclin-dependent kinase inhibitors' promoter activity and negatively affect cell growth. Cancer Res. 2000;60:1376-82.

Pastor JC, de la Rua ER, Martin F. Proliferative vitreoretinopathy: risk factors and pathobiology. Prog Retin Eye Res. 2002;21:127-44.

Peterson LE. CLUSFAVOR 5.0 hierarchical cluster and principal component analysis of microarray-based transcriptional profiles. Genome Bio. 2002;3:SOFTWARE0002.

Piatigorsky J. Multifunctional lens crystallins and corneal enzymes: more than meets the eye. Ann N Y Acad Sci. 1998;842:7-15.

Postel EA, Mieler WF. Posterior segment manifestations of blunt truma. In: Guyer DR (ed): Retina-Vitreous-Macula Vol 1. Philadelphia: W.B. Saunders Co.: 1999:831-43.

Quackenbush J. Computational analysis of microarray data. Nat Rev Genet. 2001;2:418-27.

Radner W, Sadda SR, Humayun MS, et al. Light-driven retinal ganglion cell responses in blind rd mice after neural retinal transplantation. Invest Ophthalmol Vis Sci. 2001;42:1057-65.

Rattner A, Nathans J. The genomic response to retinal disease and injury: evidence for endothelin signaling from photoreceptors to glia. J Neurosci. 2005 25:4540-9.

Reed SI. Ratchets and clocks: the cell cycle, ubiquitylation and protein turnover. Nat Rev Mol Cell Biol. 2003;4:855-64.

Reiner A, Yekutieli D, Benjamini Y. Identifying differentially expressed genes using false discovery rate controlling procedures. Bioinformatics. 2003;19:368-75.

Ridet JL, Malhotra SK, Privat A, Gage FH. Reactive astrocytes: cellular and molecular cues to biological function. Trends Neurosci. 1997;20:570-7.

Rogojina AT, Orr WE, Song BK, Geisert EE Jr. Comparing the use Affymetrix to spotted oligonucleotide microarrays using two retinal pigment epithelium cell lines. Mol Vis. 2003;9:482-96.

Ross SE, Greenberg ME, Stiles CD. Basic helix-loop-helix factors in cortical development. Neuron. 2003;39:13-25.

Ryan SJ, Stout JT, Dugel PV. Posterior penetrating ocular trauma. In: Ryan SJ (ed): Retina Vol 3. St Louis, CV Mosby Co.: 1994:2235-50.

Sahel JA, Albert DM, Lessell S, Adler H, McGee TL, Konrad-Rastegar. Mitogenic effects of excitatory amino acids in the adult rat retina. J Exp Eye Res. 1991;53:657-64. 
Sakaguchi H, Miyagi M, Darrow RM, et al. Intense light exposure changes the crystallin content in retina. Exp Eye Res. 2003;76:131-3.

Sarthy V and Ripps H. Role in retinal pathophysiology. In: The Retinal Muller Cell: Structure and Function. New York: Kluwer Academic Publishers; 2001. p. 181-215

Schadt EE, Monks SA, Drake TA, et al. Genetics of gene expression surveyed in maize, mouse and man. Nature. 2003;422:297-302.

Schauwecker PE, Steward O. Genetic determinants of susceptibility to excitotoxic cell death: implications for gene targeting approaches. Proc Natl Acad Sci U S A. 1997;94:4103-8.

Schena M, Shalon D, Davis RW, Brown PO. Quantitative monitoring of gene expression patterns with a complementary DNA microarray. Science. 1995;270:467-70.

Scott RE, White-Grindley E, Ruley HE, Chesler EJ, Williams RW. P2P-R expression is genetically coregulated with components of the translation machinery and with PUM2, a translational repressor that associates with the P2P-R mRNA. J Cell Physiol. 2005;204:99-105.

Segal E, Friedman N, Kaminski N, Regev A, Koller D. From signatures to models: understanding cancer using microarrays. Nat Genet. 2005; 37 Suppl:S38-45.

Sherry DM, Townes-Anderson E. Rapid glutamatergic alterations in the neural retina induced by retinal detachment. Invest Ophthalmol Vis Sci. 2000;41:2779-90.

Song BK, Geisert GR, Vazquez-Chona F, Geisert EE Jr. Temporal regulation of CD81 following retinal injury in the rat. Neurosci Lett. 2003;338:29-32.

Spellman PT, Sherlock G, Zhang MQ, et al. Comprehensive identification of cell cycle-regulated genes of the yeast Saccharomyces cerevisiae by microarray hybridization. Mol Biol Cell. 1998;9:3273-97.

Sullivan CD, Geisert EE Jr. Expression of rat target of the antiproliferative antibody (TAPA) in the developing brain. J Comp Neurol. 1998;396:366-80.

Stroeva OG, Mitashov VI. Retinal pigment epithelium: proliferation and differentiation during development and regeneration. Int Rev Cytol. 1983;83:221-93.

Strunnikova N, Baffi J, Gonzalez A, Silk W, Cousins SW, Csaky KG. Regulated heat shock protein 27 expression in human retinal pigment epithelium. Invest Ophthalmol Vis Sci. 2001;42:2130-8.

Szabo ME, Droy-Lefaix MT, Doly M, Carre C, Braquet P. Ischemia and reperfusion-induced histologic changes in the rat retina. Demonstration of a free radical-mediated mechanism. Invest Ophthalmol Vis Sci. 1991;32:1471-78.

Szollosi J, Horejsi V, Bene L, Angelisova P, Damjanovich S. Supramolecular complexes of MHC class I, MHC class II, CD20, and tetraspan molecules (CD53, CD81, and CD82) at the surface of a B cell line JY. J Immunol. 1996;157:2939-46.

Takeda M, Takamiya A, Yoshida A, Kiyama H. Extracellular signal-regulated kinase activation predominantly in Muller cells of retina with endotoxin-induced uveitis. Invest Ophthalmol Vis Sci. 2002;43:907-11.

Tanaka S, Suzuki K, Watanabe M, Matsuda A, Tone S, Koike T. Upregulation of a new microglial gene, mrf-1, in response to programmed neuronal cell death and degeneration. J Neurosci. 1998;18:6358-69.

Tange Y, Hirata A, Niwa O. An evolutionarily conserved fission yeast protein, Ned1, implicated in normal nuclear morphology and chromosome stability, interacts with Dis3, Pim1/RCC1 and an essential nucleoporin. J Cell Sci. 2002;115:4375-85.

Taylor BA, Wnek C, Kotlus BS, Roemer N, MacTaggart T, Phillips SJ. Genotyping new BXD recombinant inbred mouse strains and comparison of BXD and consensus maps. Mamm Genome. 1999;10:335-48. 
Toma JG, El-Bizri H, Barnabe-Heider F, Aloyz R, Miller FD. Evidence that helix-loop-helix proteins collaborate with retinoblastoma tumor suppressor protein to regulate cortical neurogenesis. J Neurosci. 2000; 20:7648-56.

Trencia A, Perfetti A, Cassese A, et al. Protein kinase B/Akt binds and phosphorylates PED/PEA-15, stabilizing its antiapoptotic action. Mol Cell Biol. 2003;23:4511-21.

Tzeng SF, Kahn M, Liva S, De Vellis J. Tumor necrosis factor-alpha regulation of the Id gene family in astrocytes and microglia during CNS inflammatory injury. Glia. 1999;26:139-52.

Tzeng SF, Bresnahan JC, Beattie MS, de Vellis J. Upregulation of the HLH Id gene family in neural progenitors and glial cells of the rat spinal cord following contusion injury. J Neurosci Res. 2001;66:1161-72.

Tzeng SF. Inhibitors of DNA binding in neural cell proliferation and differentiation. Neurochem Res. 2003;28:45-52.

Vazquez-Chona F, Song BK, Geisert EE. Temporal changes in gene expression after injury in the rat retina. Ophthalmol Vis Sci. 2004;45:2737-46.

Vazquez-Chona FR, Khan AN, Chan CK, et al. Genetic networks controlling retinal injury. Mol Vis. 2005;11:958-70.

Vidaurri-Leal J, Hohman R, Glaser BM. Effect of vitreous on morphologic characteristics of retinal pigment epithelial cells. A new approach to the study of proliferative vitreoretinopathy. Arch Ophthalmol. 1984;102:1220-3.

Vihinen T, Maatta A, Jaakkola P, Auvinen P, Jalkanen M. Functional characterization of mouse syndecan-1 promoter. J Biol Chem. 1996;271:12532-41.

Vinores SA, Derevjanik NL, Vinores MA, Okamoto N, Campochiaro PA. Sensitivity of different vascular beds in the eye to neovascularization and blood-retinal barrier breakdown in VEGF transgenic mice. Adv Exp Med Biol. 2000;476:129-38.

Wang J, Williams RW, and Manly KF. WebQTL: Web-based complex trait analysis. Neuroinformatics 2003; 1:299-308.

Wang S, Sdrulla A, Johnson JE, Yokota Y, Barres BA. A role for the helix-loop-helix protein Id2 in the control of oligodendrocyte development. Neuron. 2001;29:603-14.

Wang Y, Smith SB, Ogilvie JM, McCool DJ, Sarthy V. Ciliary neurotrophic factor induces glial fibrillary acidic protein in retinal Muller cells through the JAK/STAT signal transduction pathway. Curr Eye Res. 2002;24:305-12.

Wax MB, Tezel G. Neurobiology of glaucomatous optic neuropathy: diverse cellular events in neurodegeneration and neuroprotection. Mol Neurobiol. 2002;26:45-55.

Williams RW, Strom RC, Zhou G, Yan Z. Genetic dissection of retinal development. Semin Cell Dev Biol. 1998;9:249-55.

Willott JF, Erway LC. Genetics of age-related hearing loss in mice. IV. Cochlear pathology and hearing loss in 25 BXD recombinant inbred mouse strains. Hear Res. 1998;119:27-36.

Wilson AS, Hobbs BG, Shen WY, Speed TP, Schmidt U, Begley CG, Rakoczy PE. Argon laser photocoagulation-induced modification of gene expression in the retina. Invest Ophthalmol Vis Sci. 2003; 44:1426-34.

Wolfer DP, Crusio WE, Lipp HP. Knockout mice: simple solutions to the problems of genetic background and flanking genes. Trends Neurosci. 2002;25:336-40.

Wong P, Ulyanova T, Organisciak DT, et al. Expression of multiple forms of clusterin during light-induced retinal degeneration. Curr Eye Res. 2001;23:157-65.

Wu T, Chen Y, Chiang SK, Tso MO. NF-kappaB activation in light-induced retinal degeneration in a mouse model. Invest Ophthalmol Vis Sci. 2002;43:2834-40. 
Xi J, Farjo R, Yoshida S, Kern TS, Swaroop A, Andley UP. A comprehensive analysis of the expression of crystallins in mouse retina. Mol Vis. 2003;9:410-9.

Yang E, Lerner L, Besser D, Darnell JE Jr. Independent and cooperative activation of chromosomal c-fos promoter by STAT3. J Biol Chem. 2003; 278:15794-9.

Yang P, Agapova O, Parker A, Shannon W, Pecen P, Duncan J, Salvador-Silva M, Hernandez MR. DNA microarray analysis of gene expression in human optic nerve head astrocytes in response to hydrostatic pressure. Physiol Genomics. 2004; 17:157-69.

Yokota Y, Mori S. Role of Id family proteins in growth control. J Cell Physiol. 2002; 190:21-8.

Yoshimura N, Kikuchi T, Kuroiwa S, Gaun S. Differential temporal and spatial expression of immediate early genes in retinal neurons after ischemia-reperfusion injury. Invest Ophthalmol Vis Sci. 2003;44:2211-20.

Zhao S, Rizzolo LJ, Barnstable CJ. Differentiation and transdifferentiation of the retinal pigment epithelium. Int Rev Cytol. 1997;171:225-66.

Zhou X, Li F, Kong L, Tomita H, Li C, Cao W. Involvement of inflammation, degradation, and apoptosis in a mouse model of glaucoma. J Biol Chem. 2005;280:31240-8. 
Appendix A

Minimum Information About a Microarray Experiment (MIAME) Report 
Array design description

Array used: Affymetrix rat genome U34A (see details of this array at www.affymetrix.com)

\section{Experimental design}

1) Authors and contact information: Félix Vázquez-Chona, Bong K. Song, and Eldon E. Geisert, $\mathrm{Jr}$.

Eldon E. Geisert, Jr.

Department of Ophthalmology

University of Tennessee Health Science Center

855 Monroe Avenue

Memphis, Tennessee 38163, USA

Phone (901) 448-7740

Fax (901) 448-5028

Email: egeisert@utmem.edu

2) Type of experiment:

a. Expression of gene in normal retina vs. injured retina

b. Time course expression

3) Experimental factors:

a. Effect of injury on gene expression

b. Effect of survival time on gene expression

4) Number of hybridizations: 18

5) Quality controls:

a. The expression profiles ( $\mathrm{n}=18$ arrays) met a strict set of quality-control parameters. Housekeeping genes displayed consistent values and 5' to 3' ratios of less than 3 . The average percentage of "Affymetrix present" calls across all arrays was $40.0 \pm 1.1 \%$. The intensity profiles ( $\log _{2}$ scale) for all the arrays showed a normal distribution with a mean at $6.59 \pm 0.04$ and an average standard deviation $\left(\square^{2}\right)$ of $2.32 \pm 0.04$.

Comparison of expression profiles among arrays from independent replicates of the same experimental group ( $\mathrm{n}=3$ arrays/group) showed a within-group average correlation of $0.92 \pm 0.01$.

b. All arrays were done in triplicate with biological replicates (i.e., RNA from a separate pool of animals).

c. Real-time PCR reactions validated results for the following genes: Fos, Fosll (Fra-1), Illb, Irfl, Cryab, Crygd, Cd81, and Gfap.

6) Description of experiments: We examined gene expression levels in the normal rat retina, and injured rat retinas with 4 hours and 1, 3, 7, and 30 day survival time.

\section{Sample description}

1) Bio-source properties:

a. For gene expression studies, we used 36 adult male Sprague Dawley rats.

b. We anesthetized 36 experimental animals by intraperitoneal injection of a mixture of xylazine $(13 \mathrm{mg} / \mathrm{kg})$ and ketamine $(87 \mathrm{mg} / \mathrm{kg})$, then induced a retinal tear in both eyes of each rat. For this purpose, we used a 27 -gauge needle to penetrate the pars plana, then scraped the superior temporal retina medially to laterally. Care was taken to prevent lens and sclera damage. 
c. Twelve control animals received no injury.

d. All animals were sacrificed by intraperitoneal injection of a mixture of xylazine (26 $\mathrm{mg} / \mathrm{kg}$ ) and ketamine (174 $\mathrm{mg} / \mathrm{kg})$.

2) Biomaterial manipulations:

a. We anesthetized 36 experimental animals by intraperitoneal injection of a mixture of xylazine $(13 \mathrm{mg} / \mathrm{kg})$ and ketamine $(87 \mathrm{mg} / \mathrm{kg})$, then induced a retinal tear in both eyes of each rat. For this purpose, we used a 27-gauge needle to penetrate the pars plana, then scraped the superior temporal retina medially to laterally. Care was taken to prevent lens and sclera damage.

b. Experimental animals were split in groups of 6 and had survival times of 4 hours and $1,3,7$, and 30 days.

c. Twelve control animals received no injury.

d. Control and injured animals were sacrificed by intraperitoneal injection of a mixture of xylazine $(26 \mathrm{mg} / \mathrm{kg})$ and ketamine $(174 \mathrm{mg} / \mathrm{kg})$.

e. Retinas used for expression studies were dissected and examined under a stereomicroscope, SXZ12 (Olympus, Japan), and immediately processed.

3) Extract preparation: For total RNA extraction, we used the TRIzol method (Life Technologies, Carlsbad, CA) and confirmed the integrity of RNA with the Agilent 2100 bioanalyzer (Agilent Technologies, Palo Alto, CA).

4) cDNA synthesis, labeling, and hybridization:

a. Using Genome Explorations (Memphis, TN), we synthesized, labeled, and hybridized cRNA onto arrays according to standard Affymetrix (Santa Clara, CA) methods previously described by Rogojina et al. in Mol Vis. (2003) 9:482-96.

b. For additional information on hybridization procedures and reagents please visit www.affymetrix.com.

\section{Data analysis}

1) Measurements: We scanned arrays on the Agilent Gene Array scanner (Affymetrix).

2) Image analysis: We measured gene expression levels with Microarray Suite 5.0 (MAS 5.0; Affymetrix) using a target signal of 250. For details on the MAS 5.0 algorithms please see www.affymetrix.com. We also obtained Robust Multi-array Average (RMA) values using RMAexpress (http://stat-www.berkeley.edu/ bolstad/RMAExpress/RMAExpress.html). For details on RMA algorithms please see Irizarry RA, et al. (2003) Nucleic Acids Res. 31:e15.

3) Transformation and Normalization: The most widely used alternative transformation of the signals and ratios is the logarithm base 2 , which has the advantage of producing a continuous and broad spectrum of values and treating up- and downregulated genes in a similar fashion [Quackenbush J, (2001) Nat Rev Genet. 2:418]. For temporal analysis of the data and comparison between data extraction methods, the mean intensity for each microarray was normalized to 8 ( $\log _{2}$ scale). Total intensity normalization is a well-established method to normalize microarrays [Quackenbush J, (2001) Nat Rev Genet. 2:418; Di Giovanni S et al. (2003) Ann Neurol. 53:454]. These transformations yielded MAS 5.0 signal intensities ranging from 1 to 18 relative units of fluorescence.

4) Data filtering: To determine a "present" signal threshold in our system, we plotted the coefficient of variation versus the averaged signal value and determined the threshold signal yielding stable coefficients of variation. From this analysis, the "present" signal threshold was set at 8.64. Below this threshold, signals displayed coefficients of variation greater than $10 \%$, while their variance increased exponentially (Supplemental Fig. 2.1). Using this 
criterion, 4,480 (50.9\%) of the genes met the "present" criterion in at least one condition and were considered for further analysis.

5) Normalized MAS 5.0 and RMA comparison: We compared the "present" and normalized MAS 5.0 signals to RMA values and found an average correlation of $0.92 \pm 0.01$ (Table B.3). This result is consistent with studies comparing the standard deviation of RMA and MAS 5.0 to absolute expression [Fig. 1 in Irizarry RA, et al. Nucleic Acids Res. 2003;31:e15]. We also compared the MAS 5.0, RMA, and RT-PCR fold changes for Fos, Fosl1 (Fra-1), Illb, Irfl, Cryab, Crygd, Cd81, and Gfap at 4 hours, 3 days and 30 days after injury (Table 3 within paper). For these genes, fold changes measured with normalized MAS 5.0 and RMA values were highly similar $(r=0.89)$. Both MAS 5.0 and RMA fold changes were similar in magnitude and direction to fold changes obtained with RT-PCR. However, RT-PCR fold changes had higher correlations with normalized MAS $5.0(r=0.76)$ than with RMA $(r=$ 0.58). Together, the high correlation between normalized MAS 5.0 values and RMA values, and the similarity between microarray fold changes and RT-PCR fold changes, confirm the reliability of our "present" signals, which eliminate genes with low expression signals. Thus, we have used the normalized MAS 5.0 values for our subsequent analysis.

6) Differentially expressed genes: We used several statistical tools and stringent criteria to identify differentially expressed genes. First, we eliminated genes with low expression values from our analysis. Previous studies comparing MAS 5.0 results to serial analysis of gene expression (SAGE) results have shown that MAS 5.0 and SAGE reliably detect medium- to high-abundance transcripts in complex tissues like the brain [Evans SJ, et al. (2002) Eur J Neurosci. 16:409]. Our criterion for selecting reliable (i.e., present) signals for further analysis is described in the Results section under the heading Data extraction and normalization. Second, we considered a gene as differentially expressed if the fold change was $>1.7$ and if the change was significant at the $P<0.02$ level using Student's $t$-test (Table 2 ). Third, we determined the expected proportion of significant changes by using the false discovery rate (FDR) of Benjamini and Hochberg (Table 2). The average FDR for our injured vs. control comparisons was $21 \pm 5.1 \%$. This FDR level is consistent with studies in our lab comparing the reliability of MAS 5.0 signals to detect true biological changes [Rogojina A, et al. (2003) Mol Vis. 9:482]. Fourth, to further eliminate false positives we present only genes whose expression pattern have well-defined pattern across 5 survival times (4 hours, and 1, 3, 7, and 30 days; Fig. 2.2; Tables B.4 and B.5). Thus, looking at genes that are clustered (i.e., cooexpressed) decreases further the potential for false positives [Heyer LJ, et al. 1999 Genome Res. 9:1106].

Availability of microarray data

The raw MAS 5.0 data set is publicly available at Gene Expression Omnibus (GEO, www.ncbi.nlm.nih.gov/geo/), a gene expression and hybridization array data repository. A list of experimental conditions, samples and GEO accession numbers is provided in Table B.1. 
Appendix B

Supplemental Material For Chapter 2 
Table B.1. Experimental conditions, samples, and GEO accession numbers.

\begin{tabular}{lcc}
\hline $\begin{array}{c}\text { Experimental } \\
\text { condition }\end{array}$ & $\begin{array}{c}\text { Sample and } \\
\text { array code }\end{array}$ & Accession No. \\
\hline Control & EEG034 & GSM15760 \\
& EEG036 & GSM15761 \\
EEG094 & GSM15762 \\
& EEG078 & GSM15763 \\
& EEG081 & GSM15764 \\
1d injury & EEG082 & GSM15765 \\
& EEG076 & GSM15766 \\
& EEG079 & GSM15768 \\
3d injury & EEG080 & GSM15769 \\
& EEG069 & GSM15770 \\
& EEG070 & GSM15772 \\
7d injury & EEG075 & GSM15773 \\
& EEG011 & GSM15779 \\
& EEG012 & GSM15780 \\
30d injury & EEG077 & GSM15781 \\
& EEG018 & GSM15782 \\
& EEG019 & GSM15783 \\
& EEG093 & GSM15784 \\
\hline
\end{tabular}

The raw MAS 5.0 data set is publicly available at Gene Expression Omnibus (GEO, www.ncbi.nlm.nih.gov/geo/) under the GEO accession numbers listed here and as the group series GSE1001. Each sample represents a biological replicate with eyes pooled from 2 animals. 
Table B.2. Oligonucleotides used for real-time RT-PCR.

\begin{tabular}{|c|c|c|c|}
\hline Gene & Accession No. & Sequence $(5 \mathbb{W}$ 3D & Product (bp) \\
\hline Fos & XM_234422 & $\begin{array}{l}\text { AGAATCCGAAGGGAAAGGAA } \\
\text { GGTCATTGAGAAGAGGCAGG }\end{array}$ & 319 \\
\hline Fosll & NM_012953 & $\begin{array}{l}\text { GGATGGTGCAGCCTCATTT } \\
\text { CATCCTCCAACTTGTCGGTC }\end{array}$ & 273 \\
\hline$I l 1 b$ & NM_031512 & $\begin{array}{l}\text { ACCCAAGCACCTTCTTTTCC } \\
\text { AGACAGCACGAGGCATTTTT }\end{array}$ & 149 \\
\hline $\operatorname{Irfl}$ & NM_012591 & $\begin{array}{l}\text { CAGGGCTGAGCTGGATCAAT } \\
\text { CGGAACAGACAGGCATCCTT }\end{array}$ & 103 \\
\hline Cryab & NM_012935 & $\begin{array}{l}\text { TCCTTTCCACTCCCCAAG } \\
\text { TGTCCTTCTCCATACGCATC }\end{array}$ & 176 \\
\hline Crygd & NM_033095 & $\begin{array}{l}\text { AGCAGTGGATGGGTTTCAG } \\
\text { GTGGAATCGGTCCTGGAG }\end{array}$ & 152 \\
\hline$C d 81$ & XM_346575 & $\begin{array}{l}\text { CTGTTTGCCTGTGAGGTAGC } \\
\text { TCAGTGTGGTCAGCGTATTG }\end{array}$ & 196 \\
\hline Gfap & NM_017009 & $\begin{array}{l}\text { AGGGACAATCTCACACAGGAC } \\
\text { CTCCAGCGACTCAACCTTC }\end{array}$ & 162 \\
\hline Rps18 & XM_215328 & $\begin{array}{l}\text { CTCGCTCCTCTCCTACTTGG } \\
\text { ACCGGGTTGGTTTTGATCT }\end{array}$ & 119 \\
\hline
\end{tabular}




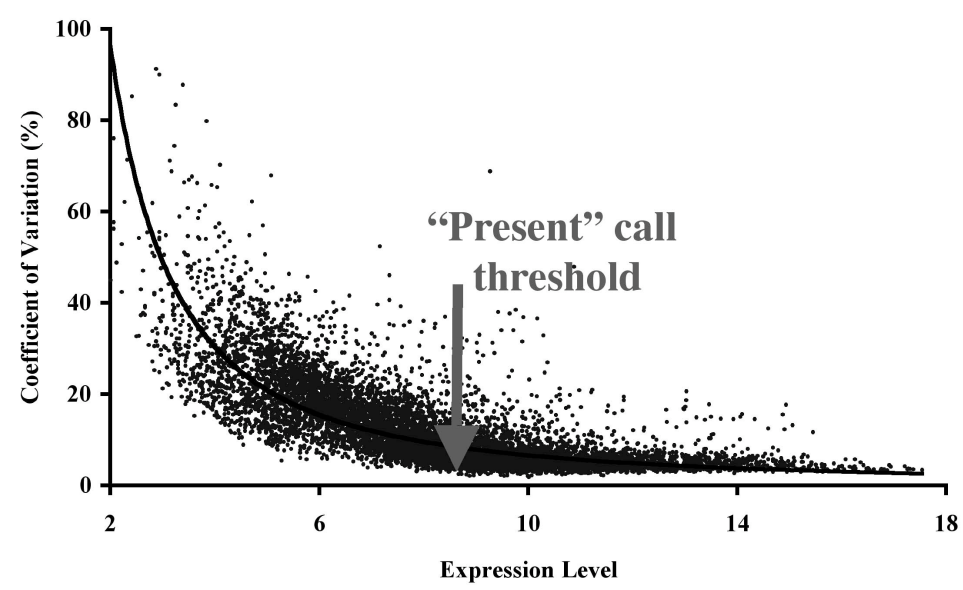

Figure B.1. Effect of expression level on coefficient of variation. To determine a "present" signal threshold in our system, we plotted the coefficient of variation versus the averaged signal value and determined the threshold signal yielding stable coefficients of variation. From this analysis, the "present" signal threshold was set at 8.64. Below this threshold, signals displayed coefficients of variation greater than $10 \%$, while their variance increased exponentially. Using this criterion, 4,480 (50.9\%) of the genes met the "present" criterion in at least one condition and were considered for further analysis. 
Table B.3. Correlation between normalized MAS 5.0 and RMA signals.

\begin{tabular}{cc}
\hline Experiment & Correlation \\
\hline Ctrl & 0.91 \\
$4 \mathrm{~h}$ & 0.91 \\
$1 \mathrm{~d}$ & 0.92 \\
$3 \mathrm{~d}$ & 0.93 \\
$7 \mathrm{~d}$ & 0.91 \\
$30 \mathrm{~d}$ & 0.93 \\
Average & $0.92 \pm 0.01$ \\
\hline
\end{tabular}


Table B.4. Gene clusters in the injured retina.

\begin{tabular}{|c|c|c|c|c|c|c|c|c|c|c|c|c|}
\hline$\Xi$ & $\tilde{F}$ & 푸 & లె & F & ప్ల & \multicolumn{7}{|c|}{ Fold Change } \\
\hline & & & & & & 1 & Pak3 & Early & 6.0 & 3.9 & 2.9 & rc_AA997367_s_at \\
\hline & & & & & & 2 & Pde4b & Early & 1.8 & 1.8 & 1.7 & rc_AA799729_g_at \\
\hline & & & & & & 3 & Plat & Early & 7.8 & 2.5 & 1.9 & M23697_at \\
\hline & & & & & & 4 & Cebpd & Early & 2.5 & 2.2 & 3.0 & M65149_at \\
\hline & & & & & & 5 & RT-BM1 & Early & 2.1 & 2.0 & 2.1 & AF029240_g_at \\
\hline & & & & & & 6 & Krt1-14* & Early & 2.4 & 2.0 & 2.5 & rc_AI639415_at \\
\hline & & & & & & 7 & Akap12 & Early & 2.3 & 1.1 & 1.6 & U41453_at \\
\hline & & & & & & 8 & Nfkb1 & Early & 3.8 & 1.7 & 2.0 & L26267_at \\
\hline & & & & & & 9 & Cxcl10 & Early & 5.5 & 1.0 & 2.9 & U17035_s_at \\
\hline & & & & & & 10 & Btg2 & Early & 2.6 & 1.2 & 1.6 & M60921_g_at \\
\hline & & & & & & 11 & Zfp3611 & Early & 2.5 & 1.5 & 1.7 & rc_AI136891_at \\
\hline & & & & & & 12 & Ssecks322 & Early & 3.6 & 2.0 & 1.6 & U75404UTR\#1_s_at \\
\hline & & & & & & 13 & Il6r & Early & 2.1 & 1.3 & 1.4 & M58587_at \\
\hline & & & & & & 14 & Kenj1 & Early & 1.9 & 1.2 & 1.1 & S78154_at \\
\hline & & & & & & 15 & EST & Early & 1.8 & 1.3 & $(1.2)$ & rc_H33084_at \\
\hline & & & & & & 16 & $\mathrm{Cbfb}$ & Early & 2.0 & 1.5 & 1.2 & AF087437UTR\#1_s_at \\
\hline & & & & & & 17 & Igfals & Early & 2.0 & 1.5 & 1.0 & S46785_at \\
\hline & & & & & & 18 & Nr4a1 & Early & 2.4 & (1.6) & (1.6) & U17254_g_at \\
\hline & & & & & & 19 & Mat2a & Early & 3.0 & 1.5 & (1.0) & J05571_s_at \\
\hline & & & & & & 20 & Arhgap7 & Early & 2.4 & 1.3 & 1.2 & D31962cds_at \\
\hline & & & & & & 21 & PDP1 & Early & 1.9 & 1.5 & 1.0 & AF062740_at \\
\hline & & & & & & 22 & Egr1 & Early & 3.4 & (2.9) & (3.4) & rc_AI176662_s_at \\
\hline & & & & & & 23 & Fos & Early & 2.6 & (1.1) & (1.1) & X06769cds_g_at \\
\hline & & & & & & 24 & Ugt1a7 & Early & 1.9 & 1.7 & 1.2 & AF039212mRNA_s_at \\
\hline & & & & & & 25 & Ndufa6* & Early & 1.7 & 1.3 & 1.1 & rc_AI229924_at \\
\hline & & & & & & 26 & EST & Early & 1.7 & 1.7 & 1.2 & U21721mRNA_at \\
\hline & & & & & & 27 & $\mathrm{Cd} 3612$ & Early & 1.7 & 1.4 & 1.2 & D10587_at \\
\hline & & & & & & 28 & Qpct & Early & 2.5 & 1.5 & $(1.0)$ & AF039308_at \\
\hline & & & & & & 29 & Mak & Early & 2.0 & 1.8 & 1.3 & M35862_at \\
\hline & & & & & & 30 & Egr1 & Early & 6.6 & (1.3) & $(1.7)$ & U75397UTR\#1_s_at \\
\hline & & & & & & 31 & Slc2a3 & Early & 2.6 & 1.4 & 1.2 & D13962_g_at \\
\hline & & & & & & 32 & Insig1 & Early & 2.5 & 1.4 & 1.2 & L13619_g_at \\
\hline & & & & & & 33 & EST & Early & 2.6 & (1.9) & (1.8) & X03347cds_g_at \\
\hline & & & & & & 34 & Cst4 & Early & 2.5 & 1.8 & 1.3 & M75281_at \\
\hline & & & & & & 35 & Crem & Early & 2.9 & $(1.2)$ & (1.4) & U04835_at \\
\hline & & & & & & 36 & Nup153 & Early & 2.1 & 1.8 & $(1.2)$ & rc_AI177052_at \\
\hline & & & & & & 37 & Insig1 & Early & 2.0 & 1.2 & 1.1 & L13619_at \\
\hline & & & & & & 38 & Jag1 & Early & 2.9 & 1.4 & 1.0 & L38483_at \\
\hline & & & & & & 39 & Stc1 & Early & 5.0 & 1.6 & 1.1 & U62667_at \\
\hline & & & & & & 40 & AF-6 & Early & 2.0 & 1.2 & 1.3 & rc_AA859702_at \\
\hline & & & & & & 41 & Gnaq & Early & 2.1 & 1.3 & 1.3 & Y17164UTR\#1_at \\
\hline & & & & & & 42 & Drd5 & Early & 1.8 & 1.4 & 1.1 & M69118cds_at \\
\hline & & & & & & 43 & Gucy2e & Early & 2.6 & 1.7 & 1.5 & L36029_at \\
\hline & & & & & & 44 & p65 & Early & 2.7 & 1.7 & 1.3 & X52772cds_at \\
\hline & & & & & & 45 & ESTs & Early & 2.2 & 1.8 & 1.5 & rc_H31078_at \\
\hline & & & & & & 46 & PLA2IR & Early & 2.3 & 1.4 & 1.2 & D30781_at \\
\hline & & & & & & 47 & Fosl1 & Early & 41.9 & 1.6 & $(1.0)$ & M19651_at \\
\hline & & & & & & 48 & Zfp3611 & Early & 2.3 & 1.1 & 1.4 & rc_AI112516_at \\
\hline & & & & & & 49 & EST & Early & 2.0 & 1.3 & 1.6 & rc_AI176491_at \\
\hline & & & & & & 50 & Pex11a & Early & 2.1 & 1.8 & 1.6 & AJ224120_at \\
\hline & & & & & & 51 & Atf3 & Early & 3.3 & 1.3 & 1.7 & M63282_at \\
\hline & & & & & & 52 & Pde4b & Early & 2.2 & 1.8 & 1.5 & M25350_s_at \\
\hline & & & & & & 53 & Igf1 & Early & 2.1 & 1.6 & 1.4 & M15481_g_at \\
\hline & & & & & & 54 & Mt1a & Early & 2.0 & (1.3) & 1.1 & rc_AI102562_at \\
\hline & & & & & & 55 & Cish3 & Early & 5.7 & 3.0 & 1.9 & AF075383_at \\
\hline & & & & & & 56 & Ctsl & Early & 2.4 & 2.1 & 1.4 & S85184_g_at \\
\hline & & & & & & 57 & ESTs & Early & 1.8 & 1.6 & 1.3 & rc_AA875633_at \\
\hline & & & & & & 58 & Bax & Early & 1.9 & 1.4 & 1.7 & S78284_s_at \\
\hline & & & & & & 59 & Rnf4 & Early & 1.8 & 2.0 & 1.2 & AF022081_at \\
\hline & & & & & & 60 & Tpm3 & Early & 3.8 & 2.2 & 1.7 & L24776_at \\
\hline & & & & & & 61 & Camk2d & Early & 2.4 & 2.5 & 1.2 & rc_AI009268_at \\
\hline & & & & & & 62 & Prkacb & Early & 3.1 & 2.7 & 1.6 & D10770_s_at \\
\hline
\end{tabular}


Table B.4. (continued). Gene clusters in the injured retina.

\begin{tabular}{|c|c|c|c|c|c|c|c|c|c|c|c|c|}
\hline \multirow{2}{*}{$\Xi$} & \multirow{2}{*}{$f$} & \multirow{2}{*}{$\Xi$} & \multirow{2}{*}{$\bar{m}$} & \multirow{2}{*}{ 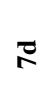 } & \multirow{2}{*}{ క్లి } & \multicolumn{7}{|c|}{ Fold Change } \\
\hline & & & & & & & Symbol & Response & Early & Delayed & Late & ProbsetID \\
\hline & & & & & & 63 & Ttgn1 & Delayed & 1.7 & 2.2 & 1.3 & X53565_at \\
\hline & & & & & & 64 & Vcam1 & Delayed $^{\dagger}$ & 5.1 & 14.0 & 7.6 & M84488_at \\
\hline & & & & & & 65 & EST & Delayed & 2.0 & 2.6 & 1.1 & rc AI 236484 at \\
\hline & & & & & & 66 & Illb & Delayed $^{\dagger}$ & 3.9 & 2.4 & 1.7 & M98820_at \\
\hline & & & & & & 67 & P2ry4 & Delayed $^{\dagger}$ & 6.6 & 4.4 & 4.2 & Y11433_s_at \\
\hline & & & & & & 68 & Tubb1 & Delayed & 1.2 & 2.3 & 1.5 & AB011679_at \\
\hline & & & & & & 69 & EST & Delayed & 1.5 & 2.0 & 1.6 & rc_AA 893743 _g_at \\
\hline & & & & & & 70 & Agtpbp $1 *$ & Delayed & 1.3 & 3.7 & 2.2 & rc_AA891864_at \\
\hline & & & & & & 71 & Stat3 & Delayed $^{\dagger}$ & 5.2 & 3.7 & 2.1 & X91810_at \\
\hline & & & & & & 72 & Dnajb9 & Delayed & 1.7 & 2.5 & 1.2 & rc_AI011998_at \\
\hline & & & & & & 73 & Pros1 & Delayed & 1.3 & 2.9 & 1.5 & U06230_s_at \\
\hline & & & & & & 74 & Irf1 & Delayed & 3.8 & 3.8 & 3.0 & M34253_g_at \\
\hline & & & & & & 75 & Ywhae & Delayed & 2.2 & 2.5 & 1.1 & rc_AA965154_at \\
\hline & & & & & & 76 & Len2 & Delayed & 9.8 & 35.5 & 28.0 & rc_AA946503_at \\
\hline & & & & & & 77 & Ptn & Delayed & 1.6 & 2.5 & 2.2 & rc_AI102795_at \\
\hline & & & & & & 78 & $\mathrm{~A} 2 \mathrm{~m}$ & Delayed & 27.9 & 54.7 & 43.0 & M22670cds_at \\
\hline & & & & & & 79 & Itgb1 & Delayed & 2.1 & 3.9 & 2.4 & rc_AI177366_at \\
\hline & & & & & & 80 & D123 & Delayed & 1.8 & 2.1 & 1.5 & U378433_g_at \\
\hline & & & & & & 81 & Dlg1 & Delayed & 1.6 & 4.3 & 1.9 & U14950_at \\
\hline & & & & & & 82 & Ar15 & Delayed & 2.1 & 2.4 & 1.5 & rc_AA 875135 at \\
\hline & & & & & & 83 & Crot & Delayed & 1.6 & 2.9 & 1.4 & U26033_at \\
\hline & & & & & & 84 & $\operatorname{Cox} 7 \mathrm{a}$ & Delayed & 2.8 & 2.6 & 1.6 & U75927ŪTR\#1_at \\
\hline & & & & & & 85 & Ywhaq & Delayed & 2.8 & 2.8 & 1.5 & rc_AI229407_at \\
\hline & & & & & & 86 & Ctsc & Delayed & 1.5 & 4.1 & 2.2 & D90404_g_at \\
\hline & & & & & & 87 & Akap11 & Delayed & 1.9 & 2.8 & 1.4 & U48288_at \\
\hline & & & & & & 88 & unr & Delayed & 2.7 & 3.0 & 1.6 & rc_AA957961_at \\
\hline & & & & & & 89 & Cntn1 & Delayed & 2.2 & 3.3 & 1.6 & D38492_at \\
\hline & & & & & & 90 & Eif5 & Delayed & 2.6 & 3.0 & 1.4 & K01677_at \\
\hline & & & & & & 91 & EST & Delayed & 1.8 & 2.4 & 1.6 & rc_AI639367_at \\
\hline & & & & & & 92 & Lman1 & Delayed & 2.0 & 3.1 & 1.5 & U44129_at \\
\hline & & & & & & 93 & Ania4 & Delayed & 8.4 & 7.8 & 2.7 & AF030089UTR\#1_at \\
\hline & & & & & & 94 & Lamp2 & Delayed & 1.8 & 3.3 & 1.9 & D90211_s_at \\
\hline & & & & & & 95 & Litaf & Delayed & 3.7 & 5.5 & 4.1 & U53184_at \\
\hline & & & & & & 96 & Pik3r3 & Delayed & 3.1 & 3.4 & 2.5 & D64047_at \\
\hline & & & & & & 97 & Eef1a2 & Delayed & 3.4 & 4.9 & 1.9 & rc_AI008852_at \\
\hline & & & & & & 98 & EST & Delayed & 2.6 & 4.5 & 2.0 & rc_AA892522_at \\
\hline & & & & & & 99 & Casp3 & Delayed & 1.8 & 4.6 & 1.9 & U459930_at \\
\hline & & & & & & 100 & Gludins & Delayed & 3.1 & 4.6 & 1.8 & D11439_s_at \\
\hline & & & & & & 101 & Casp3 & Delayed & 3.2 & 6.9 & 3.0 & $\mathrm{U} 84410 \mathrm{~s}$ at \\
\hline & & & & & & 102 & IMPG2 & Delayed & 3.5 & 6.0 & 2.1 & U76717_at \\
\hline & & & & & & 103 & $\mathrm{Gp} 38$ & Delayed & 2.4 & 3.7 & 2.2 & U92081mRNA_s_at \\
\hline & & & & & & 104 & EST & Delayed & 1.3 & 1.8 & 1.5 & rc_AA800016_at \\
\hline & & & & & & 105 & Vhl & Delayed & 1.2 & 1.8 & 1.5 & U14746_at \\
\hline & & & & & & 106 & Arl1 & Delayed & 1.2 & 2.0 & 1.4 & rc_AA875253_at \\
\hline & & & & & & 107 & Sv2b & Delayed & 1.3 & 1.7 & 1.4 & rc_AI639484_at \\
\hline & & & & & & 108 & EST & Delayed & 1.3 & 1.8 & 1.6 & rc_AA866369_at \\
\hline & & & & & & 109 & EST & Delayed & 1.2 & 2.0 & 1.3 & rc_AA891978_at \\
\hline & & & & & & 110 & Impa1 & Delayed & 1.3 & 2.4 & 1.6 & rc_AA801286_at \\
\hline & & & & & & 111 & Plrg1 & Delayed & 1.4 & 2.2 & 1.5 & rc_AI639353_at \\
\hline & & & & & & 112 & Inpp4 & Delayed & 1.3 & 1.8 & 1.1 & U96921_at \\
\hline & & & & & & 113 & Gif & Delayed & 1.9 & 4.1 & 2.0 & D45199cds_s_at \\
\hline & & & & & & 114 & Fuca & Delayed & 1.4 & 2.3 & 1.9 & X16145_at \\
\hline & & & & & & 115 & Nap111 & Delayed & 1.4 & 2.6 & 1.8 & rc_AA859920_at \\
\hline & & & & & & 116 & Ap $3 \mathrm{~m} 1$ & Delayed & 1.5 & 2.2 & 1.3 & L07073_at \\
\hline & & & & & & 117 & EST & Delayed & 1.3 & 2.1 & 1.6 & rc_AA800034_at \\
\hline & & & & & & 118 & EST & Delayed & $(1.2)$ & 1.9 & 1.5 & rc_AA859933_at \\
\hline & & & & & & 119 & Abi2 & Delayed & 1.2 & 1.8 & 1.8 & U94904_s_at \\
\hline & & & & & & 120 & EST & Delayed & 1.8 & 2.1 & 1.4 & rc_AA891220_at \\
\hline & & & & & & 121 & EST & Delayed & $(1.0)$ & 1.7 & 1.5 & rc_AA892270_g_at \\
\hline & & & & & & 122 & $\mathrm{Cd} 82$ & Delayed $^{\dagger}$ & 1.0 & 1.8 & 1.4 & rc $\mathrm{AI} 231213$ at \\
\hline & & & & & & 123 & $\mathrm{P} 4 \mathrm{hb}$ & Delayed $^{\dagger}$ & 1.1 & 1.7 & 1.5 & X02918_at \\
\hline & & & & & & 124 & Ppp2rb & Delayed & 1.2 & 2.2 & 1.5 & rc_AI103238_at \\
\hline & & & & & & 125 & $\operatorname{Lim} 2$ & Delayed $^{\dagger}$ & 1.1 & 2.5 & 2.0 & S55224_s_at \\
\hline
\end{tabular}


Table B.4. (continued). Gene clusters in the injured retina.

\begin{tabular}{|c|c|c|c|c|c|c|c|c|c|c|c|c|}
\hline \multirow{2}{*}{$\Xi$} & \multirow{2}{*}{$\tilde{F}$} & \multirow{2}{*}{$\Xi$} & \multirow{2}{*}{$\vec{m}$} & \multirow{2}{*}{$\approx$} & \multirow{2}{*}{ ల్లి } & \multicolumn{7}{|c|}{ Fold Change } \\
\hline & & & & & & & Symbol & Response & Early & Delayed & Late & ProbsetID \\
\hline & & & & & & 126 & RT1Aw2 & Late $^{\dagger}$ & $(1.0)$ & 2.0 & 1.9 & M24026_f_at \\
\hline & & & & & & 127 & $\operatorname{Lhx} 2$ & Late $^{\dagger}$ & (1.7) & 2.3 & 1.7 & L06804_at \\
\hline & & & & & & 128 & Lip1 & Late $^{\dagger}$ & (1.1) & 3.4 & 2.0 & S81497_s_at \\
\hline & & & & & & 129 & Lip1 & Late & (1.5) & 2.4 & 1.9 & S81497_i_at \\
\hline & & & & & & 130 & Tpm3 & Late & 1.5 & 2.0 & 1.9 & S82383_s_at \\
\hline & & & & & & 131 & Hsd17b4 & Late & 1.1 & 2.0 & 1.9 & rc_AI013834_s_at \\
\hline & & & & & & 132 & $\mathrm{Cp}$ & Late $^{\dagger}$ & 2.1 & 13.3 & 5.9 & rc_AA817854_s_at \\
\hline & & & & & & 133 & Gtpi* & Late $^{\dagger}$ & 2.1 & 11.4 & 5.3 & rc_AA891944_at \\
\hline & & & & & & 134 & Mx3 & Late $^{\dagger}$ & 2.7 & 4.8 & 8.7 & X $\overline{5} 2713$ _at \\
\hline & & & & & & 135 & Fcgr3 & Late $^{\dagger}$ & 1.7 & 11.5 & 6.0 & M32062_g_at \\
\hline & & & & & & 136 & Anxa5 & Late $^{\dagger}$ & 1.4 & 2.7 & 2.3 & D42137exon_s_at \\
\hline & & & & & & 137 & $\mathrm{Cd} 82$ & Late $^{\dagger}$ & 1.3 & 3.1 & 2.2 & rc_AI231213_g_at \\
\hline & & & & & & 138 & Spin2c & Late & 2.8 & 32.9 & 8.7 & D00753_at \\
\hline & & & & & & 139 & EST & Late & 1.1 & 1.5 & 1.7 & rc_AA800199_at \\
\hline & & & & & & 140 & Cntf & Late & 1.5 & 3.0 & 2.6 & rc_AA892559_at \\
\hline & & & & & & 141 & Snn & Late & (1.1) & 1.7 & 2.0 & M81639_at \\
\hline & & & & & & 142 & Aprt & Late & 1.7 & 3.2 & 3.1 & rc_AI177096_at \\
\hline & & & & & & 143 & Gsta1 & Late & 1.5 & 2.3 & 2.5 & S72505_f_at \\
\hline & & & & & & 144 & Prkr & Late & 1.3 & 3.7 & 2.6 & L29281_at \\
\hline & & & & & & 145 & $\mathrm{Cd} 81$ & Late & 1.1 & 1.8 & 1.8 & rc_AI103957_g_at \\
\hline & & & & & & 146 & Sparc & Late & 2.0 & 5.5 & 5.8 & rc_AA891204_s_at \\
\hline & & & & & & 147 & Sparc & Late & 1.6 & 3.3 & 3.3 & Y13714_at \\
\hline & & & & & & 148 & Mgp & Late & 1.3 & 3.0 & 5.5 & rc_AI012030_at \\
\hline & & & & & & 149 & Slc1a4 & Late & 1.1 & 2.8 & 3.1 & S59158_at \\
\hline & & & & & & 150 & Lyz & Late & 4.8 & 37.4 & 31.3 & rc_AA892775_at \\
\hline & & & & & & 151 & Idh2 & Late & 1.0 & 2.0 & 1.9 & rc_AA891785_g_at \\
\hline & & & & & & 152 & Arpc1b & Late & 1.4 & 4.1 & 3.2 & AF083269_g_at \\
\hline & & & & & & 153 & Slc25a20 & Late & (1.3) & 2.0 & 1.8 & rc_AA800120_at \\
\hline & & & & & & 154 & Ctsh & Late & $(1.0)$ & 2.5 & 2.4 & M38135_at \\
\hline & & & & & & 155 & Fcgr3 & Late & 1.9 & 18.9 & 13.6 & M32062_at \\
\hline & & & & & & 156 & $\mathrm{Cp}$ & Late & 2.0 & 10.2 & 9.4 & L33869_at \\
\hline & & & & & & 157 & Apoe & Late & 1.1 & 1.8 & 2.7 & X04979_at \\
\hline & & & & & & 158 & RT1.Da u & Late & 3.2 & 14.0 & 13.9 & M15562_g_at \\
\hline & & & & & & 159 & Pmp22 & Late & (1.1) & 1.8 & 2.0 & S55427_s_at \\
\hline & & & & & & 160 & Рap2b & Late & (1.3) & 4.9 & 3.5 & rc_AA924925_at \\
\hline & & & & & & 161 & $\mathrm{C} 1 \mathrm{~s}$ & Late & $(9.1)$ & 3.8 & 3.9 & D88250_at \\
\hline & & & & & & 162 & Apobec1 & Late & (1.7) & 11.8 & 5.7 & L07114_at \\
\hline & & & & & & 163 & Cd9 & Late & 1.1 & 2.7 & 2.5 & X76489cds_g_at \\
\hline & & & & & & 164 & RT1.Da u & Late & 1.3 & 6.1 & 4.7 & M15562_at \\
\hline & & & & & & 165 & Ctss & Late & (1.1) & 2.6 & 5.8 & L03201_at \\
\hline & & & & & & 166 & Cryba1 & Late & 1.1 & 6.0 & 6.2 & AF013248_at \\
\hline & & & & & & 167 & $\mathrm{Cd} 48$ & Late & $(1.3)$ & 5.3 & 3.8 & X13016_at \\
\hline & & & & & & 168 & Snca & Late & 1.5 & 2.4 & 2.6 & S73007_g_at \\
\hline & & & & & & 169 & Cryab & Late & 1.6 & 6.3 & 7.4 & X60351cds_s_at \\
\hline & & & & & & 170 & $\mathrm{C} 1 \mathrm{r}$ & Late & (1.1) & 4.2 & 5.0 & rc_AA799803_at \\
\hline & & & & & & 171 & RT1.Mb & Late & (1.3) & 6.4 & 5.7 & rc_AI171966_at \\
\hline & & & & & & 172 & Crygd & Late & (1.8) & 5.0 & 15.1 & X577169_f_at \\
\hline & & & & & & 173 & Cryge & Late & (1.4) & 3.6 & 9.0 & M19357cds_f_at \\
\hline & & & & & & 174 & $\mathrm{~A} 2 \mathrm{~m}$ & Late & 2.0 & 5.4 & 6.1 & M23566exon_s_at \\
\hline & & & & & & 175 & Ppicap & Late & $(1.1)$ & 2.5 & 2.8 & AF065438_at \\
\hline & & & & & & 176 & $\mathrm{~A} 2 \mathrm{~m}$ & Late & 1.4 & 5.7 & 5.9 & rc_AA900582_at \\
\hline & & & & & & 177 & Crygd & Late & (1.4) & 7.9 & 29.6 & X57169_i_at \\
\hline & & & & & & 178 & Ptgfr & Late & 1.5 & 4.8 & 6.0 & rc_AI170268_at \\
\hline & & & & & & 179 & Gfap & Late & 1.4 & 7.8 & 16.8 & AF028784cds\#1_s_at \\
\hline & & & & & & 180 & Crybb2 & Late & 1.3 & 7.4 & 14.7 & X83671cds_i_at \\
\hline & & & & & & 181 & Bfsp1 & Late & $(1.1)$ & 20.1 & 27.7 & AB003104_at \\
\hline & & & & & & 182 & Cryaa & Late & 1.0 & 6.5 & 8.3 & U47921_s_at \\
\hline & & & & & & 183 & Grifin & Late & 1.1 & 11.1 & 13.9 & AF082160_at \\
\hline & & & & & & 184 & $\mathrm{C} 1 \mathrm{qb}$ & Late & 1.1 & 29.1 & 72.6 & X71127_g_at \\
\hline & & & & & & 185 & RT1.C/E & Late & 1.2 & 3.5 & 5.1 & rc_AI235890_s_at \\
\hline & & & & & & 186 & Gfap & Late & 1.3 & 10.4 & 19.1 & AF028784mRNA\#1_s_at \\
\hline & & & & & & 187 & $\mathrm{Cd} 74$ & Late & $(1.0)$ & 18.5 & 37.2 & X14254cds_g_at \\
\hline & & & & & & 188 & $\operatorname{mrf}-1^{*}$ & Late & (1.3) & 13.3 & 33.7 & U10894_s_at \\
\hline & & & & & & 189 & $\mathrm{C} 3$ & Late & $(5.8)$ & 8.2 & 33.6 & M29866_s_at \\
\hline
\end{tabular}


Supplementary Table B.4. (continued). Upregulated genes and their clusters

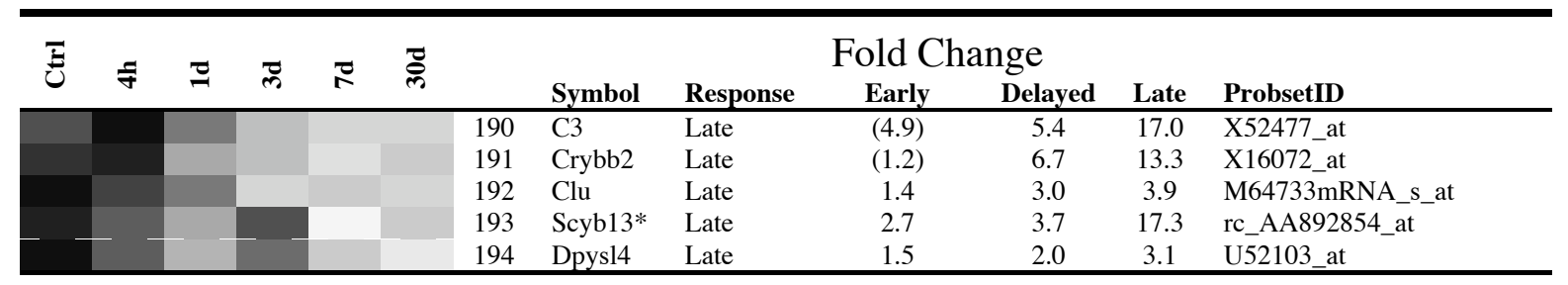

Upregulated genes (fold change $>1.7$ and $P<0.02$ ) were clustered with CLUSFAVOR 6.0 [Peterson, 2002] using principal component analysis. Using the first 3 components (at least $98 \%$ of the variability) and correlations $\geq \pm 0.6$, we identified 3 major responses: the early, delayed, and late, sustained response. The early fold change represents the expression change at 4 hours. The delayed fold change corresponds to the average change at 1 and 3 days. The late change is the averaged fold change at 7 and 30 days. Values in parentheses represent negative fold changes.

*Expressed sequence tags (ESTs) whose identity was defined by gene homology.

${ }^{\dagger}$ Clustered genes whose correlations were high on adjacent clusters.

Shades of color represent standardized levels of expression as follows:

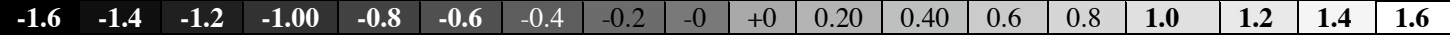


Table B.5. Gene functional clusters in injured retina

\begin{tabular}{|c|c|c|c|c|c|c|}
\hline \multirow[b]{2}{*}{ Symbol } & \multirow[b]{2}{*}{ Name } & \multirow[b]{2}{*}{ GenBank } & \multirow[b]{2}{*}{ Cluster $^{\dagger}$} & \multicolumn{3}{|c|}{ Fold Change } \\
\hline & & & & Early & Delayed & Late \\
\hline \multicolumn{7}{|c|}{1 Transcription } \\
\hline Atf3 & Activating transcription factor 3 & M63282 & E & 3.3 & 1.3 & 1.7 \\
\hline $\mathrm{Cbfb}$ & Core binding protein beta & AF087437 & E & 2.0 & 1.5 & 1.2 \\
\hline Cebpd & CCAAT/enhancer binding protein delta & M65149 & $\mathrm{E}$ & 2.5 & 2.2 & 3.0 \\
\hline Crem & CAMP responsive element modulator & U04835 & E & 2.9 & $(1.2)$ & $(1.4)$ \\
\hline Egr1 & Early growth response 1 (Krox-24) & AI176662 & E & 3.4 & (2.9) & (3.4) \\
\hline Fos & c-fos & X06769 & E & 2.6 & (1.1) & (1.1) \\
\hline Fosl1 & Fos-like antigen 1 (Fra-1) & M19651 & $\mathrm{E}$ & 41.9 & 1.6 & $(1.0)$ \\
\hline Irf1 & Interferon regulatory factor 1 & M34253 & $\mathrm{D}$ & 3.8 & 3.8 & 3.0 \\
\hline Isl1 & ISL1 transcription factor & S69329 & E & 1.7 & 1.7 & 1.7 \\
\hline Lhx2 & LIM homeobox protein 2 & L06804 & $\mathrm{D}, \mathrm{L}$ & (1.7) & 2.3 & 1.7 \\
\hline $\mathrm{Nfkb} 1$ & Nuclear factor kappa B p105 & L26267 & $\mathrm{E}$ & 3.8 & 1.7 & 2.0 \\
\hline $\mathrm{Nr} 4 \mathrm{a} 1$ & Nuclear receptor subfamily $4 \mathrm{a} 1$ & U17254 & $\mathrm{E}$ & 2.4 & $(1.6)$ & $(1.6)$ \\
\hline Plrg1 & Pleiotropic regulator 1 & AI639353 & $\mathrm{D}$ & 1.4 & 2.1 & 1.5 \\
\hline Rnf4 & Ring finger protein 4 & AF022081 & $\mathrm{E}$ & 1.8 & 2.0 & 1.2 \\
\hline Stat3 & Stat3 & X91810 & $\mathrm{D}$ & 5.2 & 3.7 & 2.1 \\
\hline Zfp3611 & Zinc finger protein $36, \mathrm{C} 3 \mathrm{H}$ type-like 1 & AI136891 & $\mathrm{E}$ & 2.5 & 1.5 & 1.7 \\
\hline \multicolumn{7}{|c|}{2 Cell cycle and growth } \\
\hline Abi2 & abl-interactor 2 & U94904 & $\mathrm{D}$ & 1.2 & 1.8 & 1.8 \\
\hline Akap11 & A kinase anchor protein 11 & U48288 & $\mathrm{D}$ & 1.9 & 2.7 & 1.4 \\
\hline Akap12 & A kinase anchor protein 12 & U41453 & $\mathrm{E}$ & 2.3 & 1.1 & 1.6 \\
\hline Arhgap7 & Rho GTPase activating protein 7 & D31962 & $\mathrm{E}$ & 2.4 & 1.3 & 1.2 \\
\hline Btg2 & B-cell translocation gene 2 & M60921 & $\mathrm{E}$ & 2.6 & 1.2 & 1.6 \\
\hline $\mathrm{Cd} 82$ & Cd82 (Kai1) & AI231213 & $\mathrm{D}, \mathrm{L}$ & 1.3 & 3.1 & 2.2 \\
\hline D123 & Cell cycle related D123 & U34843 & $\mathrm{D}$ & 1.8 & 2.1 & 1.5 \\
\hline Dlg1 & Discs-large homolog 1 & U14950 & $\mathrm{D}$ & 1.6 & 4.3 & 1.9 \\
\hline Igf1 & Insulin-like growth factor 1 & M15481 & $\mathrm{E}$ & 2.1 & 1.6 & 1.4 \\
\hline Igfals & IGF binding protein complex acid-labile & S46785 & $\mathrm{E}$ & 2.0 & 1.5 & 1.0 \\
\hline Insig1 & Growth response protein CL-6 & L13619 & $\mathrm{E}$ & 2.5 & 1.4 & 1.2 \\
\hline Itgb1 & Integrin, beta 1 & AI177366 & $\mathrm{D}$ & 2.1 & 3.8 & 2.4 \\
\hline Len2 & Lipocalin 2 & AA946503 & $\mathrm{D}$ & 9.8 & 35.5 & 28.0 \\
\hline Nap111 & Nucleosome assembly protein 1 -like 1 & AA859920 & $\mathrm{D}$ & 1.4 & 2.6 & 1.8 \\
\hline Pak3 & p21 CDKN1A activated kinase 3 & AA997367 & $\mathrm{E}$ & 6.0 & 3.9 & 2.9 \\
\hline Ppap2b & Phosphatidic acid phosphatase type $2 \mathrm{~B}$ & AA924925 & $\mathrm{L}$ & $(1.3)$ & 4.9 & 3.5 \\
\hline Prkr & interferon-inducible Eif2ak2 & L29281 & $\mathrm{L}$ & 1.3 & 3.7 & 2.6 \\
\hline Ptn & Pleiotrophin (Hbnf) & AI102795 & $\mathrm{D}$ & 1.6 & 2.5 & 2.2 \\
\hline Ssecks322 & Src- and ras-suppressed gene, 322 & U75404 & $\mathrm{E}$ & 3.6 & 2.0 & 1.5 \\
\hline Vhl & Von Hippel-Lindau syndrome & U14746 & $\mathrm{D}$ & 1.2 & 1.8 & 1.5 \\
\hline Ywhae & $14-3-3 E$ & AA965154 & $\mathrm{D}$ & 2.2 & 2.5 & 1.1 \\
\hline Ywhaq & $14-3-3 Q$ & AI229407 & $\mathrm{D}$ & 2.8 & 2.8 & 1.5 \\
\hline \multicolumn{7}{|c|}{3 Apoptosis and cell death } \\
\hline Apobec1 & Apolipoprotein B editing protein & L07114 & $\mathrm{L}$ & $(1.7)$ & 11.8 & 5.7 \\
\hline Bax & Bcl2-associated X protein & S78284 & $\mathrm{E}$ & 1.9 & 1.4 & 1.7 \\
\hline Casp3 & Caspase 3 & U49930 & $\mathrm{D}$ & 1.8 & 4.6 & 1.8 \\
\hline Ctsl & Cathepsin L & S85184 & $\mathrm{E}$ & 2.4 & 2.1 & 1.4 \\
\hline Eefla2 & Euk. translation elongation factor $1 \mathrm{a} 2$ & AI008852 & $\mathrm{D}$ & 3.4 & 4.9 & 1.9 \\
\hline Litaf & LPS-induced TNF-alpha factor & U53184 & $\mathrm{D}$ & 3.7 & 5.5 & 4.1 \\
\hline Mx3 & Myxovirus resistance 3 & X52713 & $\mathrm{D}, \mathrm{L}$ & 2.7 & 4.8 & 8.7 \\
\hline Tnfrsf $21 *$ & TNF receptor $21 *$ & AA891842 & $\mathrm{D}$ & 1.2 & 1.6 & 2.0 \\
\hline Vdac1 & Voltage-dependent anion channel 1 & AF048828 & $\mathrm{E}, \mathrm{L}$ & 1.7 & 1.2 & 1.8 \\
\hline
\end{tabular}


Table B.5. (continued). Gene functional clusters in injured retina

\begin{tabular}{|c|c|c|c|c|c|c|}
\hline \multirow[b]{2}{*}{ Symbol } & \multirow[b]{2}{*}{ Name } & \multirow[b]{2}{*}{ GenBank } & \multirow[b]{2}{*}{ Cluster $^{\dagger}$} & \multicolumn{3}{|c|}{ Fold Change } \\
\hline & & & & Early & Delayed & Late \\
\hline \multicolumn{7}{|c|}{4 Immune/inflammation/Stress } \\
\hline $\mathrm{C} 1 \mathrm{qb}$ & Complement component $1 \mathrm{qb}$ & X71127 & $\mathrm{L}$ & 1.1 & 29.1 & 72.6 \\
\hline $\mathrm{C} 1 \mathrm{r}$ & Complement component $1 \mathrm{r}^{*}$ & AA799803 & $\mathrm{L}$ & $(1.1)$ & 4.2 & 5.0 \\
\hline $\mathrm{C} 1 \mathrm{~s}$ & Complement component $1 \mathrm{~s}$ & D88250 & $\mathrm{L}$ & $(9.1)$ & 3.8 & 3.9 \\
\hline $\mathrm{C} 3$ & Complement component 3 & $\mathrm{X} 52477$ & $\mathrm{~L}$ & (4.9) & 5.4 & 17.0 \\
\hline $\mathrm{Cd} 48$ & $\mathrm{Cd} 48$ & X13016 & $\mathrm{L}$ & (1.3) & 5.3 & 3.8 \\
\hline $\mathrm{Cd} 74$ & $\mathrm{Cd} 74$ & X14254 & $\mathrm{L}$ & $(1.0)$ & 18.5 & 37.2 \\
\hline Cish3 & Cytokine inducible SH2-containing 3 & AF075383 & $\mathrm{E}$ & 5.7 & 3.0 & 1.8 \\
\hline Cst4 & Cystatin S & M75281 & $\mathrm{E}$ & 2.5 & 1.8 & 1.2 \\
\hline Ctsc & Cathepsin $\mathrm{C}$ & D90404 & $\mathrm{D}$ & 1.5 & 4.1 & 2.2 \\
\hline Cxcl10 & Chemokine (C-X-C motif) ligand 10 & U17035 & $\mathrm{E}$ & 5.5 & 1.0 & 2.9 \\
\hline Dnajb9 & DnaJ (Hsp40) homolog, subfamily b9 & AI011998 & $\mathrm{D}$ & 1.7 & 2.5 & 1.2 \\
\hline Edn2 & Endothelin-2 & U59510 & $\mathrm{L}$ & 2.1 & 2.9 & 5.1 \\
\hline Fcgr3 & Fc receptor $\operatorname{IgG}$, low affinity 3 & M32062 & $\mathrm{D}, \mathrm{L}$ & 1.9 & 18.9 & 13.6 \\
\hline Fcgr3 & Fc receptor IgG, low affinity 3 & M32062 & $\mathrm{D}, \mathrm{L}$ & 1.7 & 11.5 & 6.0 \\
\hline Gsta1 & Glutathione-S-transferase, alpha 1 & S72505 & $\mathrm{L}$ & 1.5 & 2.3 & 2.5 \\
\hline Gtpi* & Interferon-g induced GTPase* & AA891944 & $\mathrm{D}, \mathrm{L}$ & 2.1 & 11.4 & 5.3 \\
\hline Hspa1a & Heat shock 70kD 1A & AA818604 & $\mathrm{L}$ & 1.6 & 1.7 & 2.1 \\
\hline Ifitm31 & Interferon induced transmembrane 31 & X61381 & $\mathrm{D}, \mathrm{L}$ & 2.4 & 4.3 & 3.9 \\
\hline $\mathrm{Il} 1 \mathrm{~b}$ & Interleukin 1 , beta & M98820 & $\mathrm{D}$ & 3.9 & 2.4 & 1.7 \\
\hline $\mathrm{Il} 2 \mathrm{rb}$ & Interleukin 2 receptor beta (CD122) & M55050 & $\mathrm{E}$ & 5.5 & 1.3 & 3.8 \\
\hline Il6r & Interleukin 6 receptor & M58587 & $\mathrm{E}$ & 2.1 & 1.2 & 1.4 \\
\hline Lman1 & Lectin mannose-binding 1 & U44129 & $\mathrm{D}$ & 2.0 & 3.1 & 1.5 \\
\hline Mtla & Metallothionein & AI102562 & $\mathrm{E}$ & 2.0 & (1.3) & 1.1 \\
\hline Pros 1 & Protein S & U06230 & $\mathrm{D}$, & 1.3 & 2.9 & 1.5 \\
\hline Ptgs 2 & Prostaglandin synthase 2 (COX-2) & S67722 & $\mathrm{E}$ & 2.1 & (2.6) & $(3.2)$ \\
\hline RT1.C/E & MHC class Ib (RT1.C/E) & AI235890 & $\mathrm{L}$ & 1.2 & 3.5 & 5.1 \\
\hline RT1.Da u & MHC class IIa RT1.u-D-alpha & M15562 & $\mathrm{L}$ & 3.2 & 14.0 & 13.9 \\
\hline $\mathrm{RT} 1 . \mathrm{Mb}$ & MHC class II DM beta & AI171966 & $\mathrm{L}$ & $(1.3)$ & 6.4 & 5.7 \\
\hline RT1Aw2 & MHC class Ib alpha & M24026 & $\mathrm{D}, \mathrm{L}$ & $(1.0)$ & 2.0 & 1.9 \\
\hline RT-BM1 & MHC class Ib BM1k antigen & AF029240 & $\mathrm{E}$ & 2.1 & 2.0 & 2.1 \\
\hline Scyb13* & Small inducible cytokine B13* & AA892854 & $\mathrm{L}$ & 2.7 & 3.7 & 17.3 \\
\hline Snn & Stannin & M81639 & $\mathrm{L}$ & $(1.1)$ & 1.7 & 2.0 \\
\hline Sod2 & Superoxide dimutase 2 mitochondrial & Y00497 & $\mathrm{E}$ & 2.6 & 2.0 & 1.8 \\
\hline \multicolumn{7}{|c|}{5 Neural development/degeneration \& Gliosis } \\
\hline $\mathrm{A} 2 \mathrm{~m}$ & Alpha-2-macroglobulin & AA900582 & $\mathrm{D}, \mathrm{L}$ & 1.4 & 5.7 & 5.8 \\
\hline Ania4 & Activity \& neurotransmitter-induced 4 & AF030089 & $\mathrm{D}$ & 8.4 & 7.8 & 2.7 \\
\hline Anxa5 & Annexin V & D42137 & $\mathrm{L}$ & 1.4 & 2.7 & 2.3 \\
\hline Apoe & Apolipoprotein E & X04979 & $\mathrm{L}$ & 1.1 & 1.7 & 2.7 \\
\hline $\mathrm{Cd} 81$ & Cd81 (TAPA) & AI103957 & $\mathrm{L}$ & 1.1 & 1.8 & 1.8 \\
\hline Clu & Clusterin & M64733 & $\mathrm{L}$ & 1.4 & 3.0 & 3.9 \\
\hline Cntf & Cntf & AA892559 & $\mathrm{L}$ & 1.5 & 3.0 & 2.6 \\
\hline Cntn1 & Contactin 1 & D38492 & $\mathrm{D}$ & 2.2 & 3.3 & 1.6 \\
\hline $\mathrm{Cp}$ & Ceruloplasmin (ferroxidase) & L33869 & $\mathrm{L}$ & 2.0 & 10.2 & 9.4 \\
\hline Cryab & Crystallin alpha B & M55534 & $\mathrm{L}$ & 1.9 & 6.0 & 4.7 \\
\hline Cstb & Cystatin beta & AI008888 & $\mathrm{E}$ & 1.5 & 1.7 & 1.3 \\
\hline Dpys14 & Dihydropyrimidinase-like 4 (Crmp3) & U52103 & $\mathrm{L}$ & 1.5 & 2.0 & 3.1 \\
\hline Drd5 & Dopamine receptor D5 & M69118 & $\mathrm{E}$ & 1.8 & 1.4 & 1.1 \\
\hline Gfap & Gfap & AF028784 & $\mathrm{L}$ & 1.4 & 7.8 & 16.8 \\
\hline Jag1 & Jagged 1 & L38483 & $\mathrm{E}$ & 2.9 & 1.4 & 1.0 \\
\hline mrf-1* & Microglia response factor (Aif1)* & U10894 & $\mathrm{L}$ & $(1.3)$ & 13.3 & 33.7 \\
\hline
\end{tabular}


Table B.5. (continued). Gene functional clusters in injured retina

\begin{tabular}{|c|c|c|c|c|c|c|}
\hline \multirow[b]{2}{*}{ Symbol } & \multirow[b]{2}{*}{ Name } & \multirow[b]{2}{*}{ GenBank } & \multicolumn{4}{|c|}{ Fold Change } \\
\hline & & & Cluster $^{\dagger}$ & Early & Delayed & Late \\
\hline Ninj1 & Ninjurin & U72660 & $\mathrm{D}$ & 1.3 & 1.5 & 1.4 \\
\hline Pea15 & Phosphoprotein in astrocyte 15 & AA894345 & $\mathrm{L}$ & 1.0 & 1.4 & 1.8 \\
\hline Pmp22 & Peripheral myelin protein 22 (Gas-3) & S55427 & $\mathrm{L}$ & $(1.1)$ & 1.8 & 2.0 \\
\hline S100a1 & S100 A1 & U26356 & $\mathrm{L}$ & 1.3 & 1.3 & 1.7 \\
\hline Snca & Synuclein 1 & S73007 & $\mathrm{L}$ & 1.5 & 2.4 & 2.5 \\
\hline Sparc & Sparc (osteonectin) & AA891204 & $\mathrm{L}$ & 2.0 & 5.5 & 5.8 \\
\hline Spin $2 c$ & Serine protease inhibitor & D00753 & $\mathrm{L}$ & 2.8 & 32.9 & 8.7 \\
\hline Vcam1 & Vcam 1 & M84488 & $\mathrm{D}$ & 5.1 & 14.0 & 7.6 \\
\hline \multicolumn{7}{|c|}{6 Structure \& Tissue remodeling } \\
\hline Arpc1b & Actin-related protein complex $1 \mathrm{~b}$ & AF083269 & $\mathrm{L}$ & 1.4 & 4.1 & 3.2 \\
\hline Bfsp1 & Filensin & AB003104 & $\mathrm{L}$ & $(1.1)$ & 20.1 & 27.7 \\
\hline Ctsh & Cathepsin $\mathrm{H}$ & M38135 & $\mathrm{L}$ & $(1.0)$ & 2.5 & 2.4 \\
\hline Ctss & Cathepsin S & L03201 & $\mathrm{L}$ & (1.1) & 2.6 & 5.8 \\
\hline Fmod & Fibromodulin & X82152 & $\mathrm{L}$ & 1.2 & 3.9 & 5.4 \\
\hline Fuca & Fucosidase alpha-L-1 tissue & $\mathrm{X} 16145$ & $\mathrm{D}, \mathrm{L}$ & 1.4 & 2.3 & 1.9 \\
\hline Grifin & Galectin-related inter-fiber & AF082160 & $\mathrm{L}$ & 1.1 & 11.1 & 13.9 \\
\hline IMPG2 & Proteoglycan 10.2 & U76717 & $\mathrm{D}$ & 3.5 & 6.0 & 2.1 \\
\hline Krt1-14* & Keratin complex-1, acidic, gene $14^{*}$ & AI639415 & E & 2.4 & 1.9 & 2.5 \\
\hline Lim2 & Lens intrinsic membrane protein 2 & S55224 & $\mathrm{D}$ & 1.1 & 2.5 & 2.0 \\
\hline Lip1 & Lysosomal acid lipase & S81497 & $\mathrm{D}, \mathrm{L}$ & $(1.5)$ & 2.4 & 1.8 \\
\hline Lyz & Lysozyme & AA892775 & $\mathrm{L}$ & 4.8 & 37.4 & 31.3 \\
\hline Mgp & Matrix Gla protein & AI012030 & $\mathrm{L}$ & 1.3 & 3.0 & 5.5 \\
\hline Myo5b & Myosin Vb & U60416 & $\mathrm{L}$ & 2.2 & 2.7 & 2.2 \\
\hline Nedd4a & Ubiquitin ligase & U50842 & $\mathrm{L}$ & 1.5 & 1.9 & 1.9 \\
\hline Plat & Plasminogen activator tissue & M23697 & $\mathrm{E}$ & 7.8 & 2.5 & 1.9 \\
\hline Tpm3 & Tropomyosin 3 gamma & L24776 & $\mathrm{E}$ & 3.8 & 2.2 & 1.7 \\
\hline Tpm3 & Tropomyosin isoform 3 & S82383 & $\mathrm{L}$ & 1.5 & 2.0 & 1.9 \\
\hline Tubb1 & Tubulin, beta 5 & AB011679 & $\mathrm{D}$ & 1.2 & 2.3 & 1.5 \\
\hline \multicolumn{7}{|l|}{7 Crystallins } \\
\hline Cryaa & Crystallin alpha A & U47921 & $\mathrm{L}$ & 1.0 & 6.5 & 8.3 \\
\hline Cryab & Crystallin alpha B & M55534 & $\mathrm{L}$ & 1.9 & 6.0 & 4.7 \\
\hline Crybal & Crystallin beta $\mathrm{A} 1 / \mathrm{A} 3$ & AF013248 & $\mathrm{L}$ & 1.1 & 6.0 & 6.2 \\
\hline Cryba4 & Crystallin beta $\mathrm{A} 4^{\S}$ & AF013247 & & $(1.1)$ & 5.9 & 8.8 \\
\hline Crybb1 & Crystallin beta $\mathrm{B} 1^{\S}$ & X06377 & & $(1.2)$ & 6.5 & 7.2 \\
\hline Crybb2 & Crystallin beta B2 & X83671 & $\mathrm{L}$ & 1.3 & 7.4 & 14.7 \\
\hline Crybb3 & ${\text { Crystallin beta } \mathrm{B} 3^{\S}}^{\S}$ & M15901 & & 1.3 & 4.9 & 7.4 \\
\hline Crygc & Crystallin gamma $C^{\S}$ & J00717 & & $(1.6)$ & 5.6 & 14.3 \\
\hline Crygd & Crystallin gamma D & X57169 & $\mathrm{L}$ & (1.8) & 5.0 & 15.1 \\
\hline Cryge & Crystallin gamma $\mathrm{E}$ & M19357 & $\mathrm{L}$ & (1.4) & 3.6 & 9.0 \\
\hline
\end{tabular}

Differentially expressed genes that met the clustering criteria (see Figure 3.2 and Table B.4) were classified into 11 functional categories. The list here illustrates the major changes in gene expression, and excludes genes in the following functional categories: signal transduction, metabolism and transport/transmission, and unknown. The early fold change represents the expression change at 4 hours. The delayed fold change corresponds to the average change at 1 and 3 days. The late change is the averaged fold change at 7 and 30 days.

*Expressed sequence tags (ESTs) whose identity was defined by gene homology.

Clusters E, D, and L represent the early, delayed, and late response, respectively.

${ }^{\S}$ Differentially expressed genes that are at the margin of filtering threshold and discussed in the Results and Discussion sections. 
Appendix C

Supplemental Material for Chapter 4 
Table C.1. Wound-healing genes modulated by chromosome 12 locus.

\begin{tabular}{|c|c|c|c|c|c|c|c|c|}
\hline \multirow[b]{2}{*}{ U34 Probe Set ID } & \multirow[b]{2}{*}{ Symbol } & \multicolumn{3}{|c|}{ Fold Change } & \multicolumn{3}{|c|}{ Cellular Expression } & \multirow[b]{2}{*}{ U74 Probe Set ID } \\
\hline & & Early & Delayed & Chronic & Muller & Astrocyte & Neuron & \\
\hline rc_AI639153_at & Acrvl & $(1.2)$ & $(1.6)$ & $(2.2)$ & + & - & + & 92897_at \\
\hline L14680_g_at & $b c l 2$ & (1.4) & (1.4) & $(1.2)$ & + & + & + & 98868_at \\
\hline U49930_at & casp3 & 1.8 & 4.6 & 1.8 & - & ++ & ++ & 98436_s_at \\
\hline U22414_at & $\operatorname{Ccl3}($ Scya3) & 3.4 & $(1.7)$ & $(1.8)$ & ++ & + & ++ & 102424_at \\
\hline rc_AI103957_g_at & $C d 81$ & 1.0 & 1.8 & 1.8 & +++ & ++ & +++ & 101495_at \\
\hline U75405UTR\#1_f_at & Collal & 1.5 & 12.5 & 37.8 & + & +++ & +++ & 103709_at \\
\hline L33869_at & $C p$ & 2.0 & 10.1 & 9.4 & +++ & + & ++ & 92851_at \\
\hline S66024_g_at & Crem & 2.7 & 1.7 & 1.5 & ++ & + & + & 160526_s_s_at \\
\hline U02553cds_s_at & Duspl (Ptpn16) & 4.2 & $(1.7)$ & $(2.1)$ & ++ & +++ & ++ & 104598_at \\
\hline rc_AI176662_s_at & Egrl & 3.4 & (2.9) & $(3.4)$ & ++ & + & ++ & 161802_i_at \\
\hline L20681_at & Etsl & $(1.8)$ & (1.7) & $(2.2)$ & + & + & + & $94720 \_$at \\
\hline X06769cds_g_at & Fos & 2.6 & $(1.1)$ & $(1.1)$ & +++ & +++ & +++ & 160901_at \\
\hline AF028784mRNA\#1_s_at & Gfap & 1.3 & 10.4 & 19.1 & +++ & ++ & + & 94143_at \\
\hline M57672mRNA\#2_s_at & Ggt1 & $(1.2)$ & $(1.5)$ & $(2.1)$ & + & + & + & 100085_at \\
\hline D13418_at & Hes3 & (1.5) & $(2.0)$ & $(2.2)$ & + & + & + & 97156_at \\
\hline D26393exon_s_at & $H k 2$ & (1.4) & (3.3) & (1.5) & ++ & + & - & 161313_at \\
\hline rc_AI230256_at & $\operatorname{Id} 2$ & 6.3 & 3.2 & 2.2 & ++ & +++ & +++ & 93013_at \\
\hline rc_AI177366_at & Itgbl & 2.1 & 3.9 & 2.4 & ++ & +++ & +++ & 100123_f_at \\
\hline X54686cds_at & Junb & 1.5 & $(2.3)$ & $(1.2)$ & +++ & + & + & 102362_i_at \\
\hline X94551_at & Lamcl & $(1.2)$ & (1.4) & (1.7) & + & + & - & 102404_at \\
\hline D90211_s_at & Lamp2 & 1.8 & 3.3 & 1.9 & ++ & ++ & +++ & 101590_at \\
\hline rc_AA 892775 at & Lyz & 4.8 & 37.5 & 31.3 & + & - & + & 100611_at \\
\hline rc_AA963674_at & $\operatorname{Map} 2 k 2$ & $(1.1)$ & $(1.5)$ & $(1.3)$ & +++ & ++ & ++ & 92543_at \\
\hline D82074_g_at & Neurodl & 1.7 & 2.4 & 1.3 & + & ++ & + & 92717_at \\
\hline rc_AI176488_at & $N f i b$ & $(2.1)$ & $(1.2)$ & $(1.2)$ & ++ & +++ & +++ & 99440_at \\
\hline U17254_at & Nr4al & 2.7 & (1.4) & (1.3) & ++ & ++ & ++ & 102371_at \\
\hline S74393_s_at & Pax6 & 1.7 & 2.4 & 1.4 & + & ++ & ++ & 92271_at \\
\hline U17013_at & Pou 2fl & $(1.3)$ & $(2.1)$ & $(1.0)$ & + & + & + & 102893_at \\
\hline U04740_at & Ptafr & 1.2 & (1.3) & (1.6) & + & ++ & + & 94158_f_at \\
\hline rc_AI170268_at & Ptgfr & 1.5 & 4.8 & 5.9 & +++ & + & + & 161713_f_at \\
\hline M83680_at & Rabl4 & $(2.4)$ & $(2.6)$ & $(1.5)$ & + & +++ & +++ & 97301_at \\
\hline L19698_at & Rala & $(1.7)$ & $(2.2)$ & (1.5) & + & +++ & ++ & 94998_at \\
\hline rc_AA799724_at & Rpol & $(2.1)$ & (1.9) & $(2.2)$ & - & ++ & ++ & 161347_r_at \\
\hline S61865_s_at & $S d c 1$ & $(56.5)$ & $(5.0)$ & (7.2) & + & ++ & + & 161370_f_at \\
\hline S59893_f_at & $S s b$ & 1.1 & 1.0 & (1.6) & + & ++ & ++ & 92579_at \\
\hline X91810_at & Stat3 & 5.2 & 3.7 & 2.1 & + & ++ & ++ & 99099_at \\
\hline U09228_at & $T c f 4$ & (1.6) & $(1.5)$ & $(1.2)$ & ++ & +++ & +++ & 160483_at \\
\hline rc_H31287_g_at & Trib3 & $(1.2)$ & (2.7) & 1.1 & + & + & + & 161067_at \\
\hline AF 048828 g_at & Vdacl & 1.6 & (1.1) & 1.7 & ++ & ++ & ++ & 98139_at \\
\hline rc_AI136891_at & Zfp36l1 & 2.5 & 1.5 & 1.7 & +++ & ++ & +++ & 93324_at \\
\hline
\end{tabular}




\title{
Retinal Injury
}

\begin{tabular}{lcl} 
Activation & De-differentiation & Remodeling \\
\hline Acute phase & Sub-acute phase & Chronic phase \\
Fos, Egr1, Nr4a1, & Id2, Pax6, Neurod1, Crem, & Ptgfr, Gfap, Cp, Col1a1, \\
Junb, Dusp1, Stat3 & Lamp2, Casp3, Itgb1 & Cd81, Lyz \\
Zfp3611, Id2
\end{tabular}

Nfib, Rala, Rab14, Bcl2, Hk2,

Tcf4, Pou2f1, Hes3, Ssb, Sox 11

\section{Retinal Development}

\section{Proliferation Differentiation}

\begin{abstract}
P4
\end{abstract}
Zfp3611, Id2, Pax6, Tcf4, Ssb1, Nfib, Casp3, Sox11

\section{Differentiation}

$\begin{array}{llll}\text { P8 } & \text { P10 } & \text { P12 } & \text { P14 }\end{array}$

\section{Maturation}

\section{P21 Adult}

\author{
Rala, Rab14, Lamp2, Dusp, Stat3, Gfap, Junb, Fos, \\ Ssb1, Itgb1, Casp3, Lyzs \\ Ets1, Egr1, Pou2f1, Bcl2, \\ Hes3
}

Crem, Lpin1, Cd81, Mapk2k2,

Nr4a1, Egr1, Neurod1

Trib3, Acrv1, Lamc1, Bcl2, Rpo1-1, Fos, Rab14, Casp3

Figure C.1. Expression patterns for chromosome 12 network genes during retinal development and retinal injury. A survey of temporal expression during retinal development [Dorell et al., 2004] and wound healing [Vazquez-Chona et al., 2004] is helpful in establishing flows of action within the network. For example the genes involved in regulating proliferation and migration in the postnatal retina (postnatal days zero through four) include Id2, Zfp361l, Pax6, Tcf4, Ssb1, $N f i b, C a s p 3$, and Rala; whereas genes involved in Muller glia differentiation (post-natal days eight through fourteen) include the glial genes Stat3, Gfap, Ets1, and Hes3. The pattern of expression in the injured retina reveal that most of the chromosome 12 network genes are differentially expressed during the early acute and delayed subacute phases of wound healing. Network genes underlying the acute phase include the transcription regulators Id2, Zfp36l1, Fos, Egrl, Nr4al, Junb, and Stat3. Network genes underlying the subacute response include xxxx. A select number of network genes are also differentially expressed during the chronic response. The chronic response is characterized by tissue remodeling including retinal re-wiring and the formation of ectopic glial scar tissue. Chronic genes in the network include the glial markers $G$ fap, $C p$, and $C d 81$. Expression patterns of the network genes during development and wound healing support the hypothesis that the chromosome 12 locus regulates early wound-healing events such as the activation of pathways that lead to apoptosis, proliferation, and dedifferentiation of cells. 
Appendix D

Supplemental Material for Chapter 5 

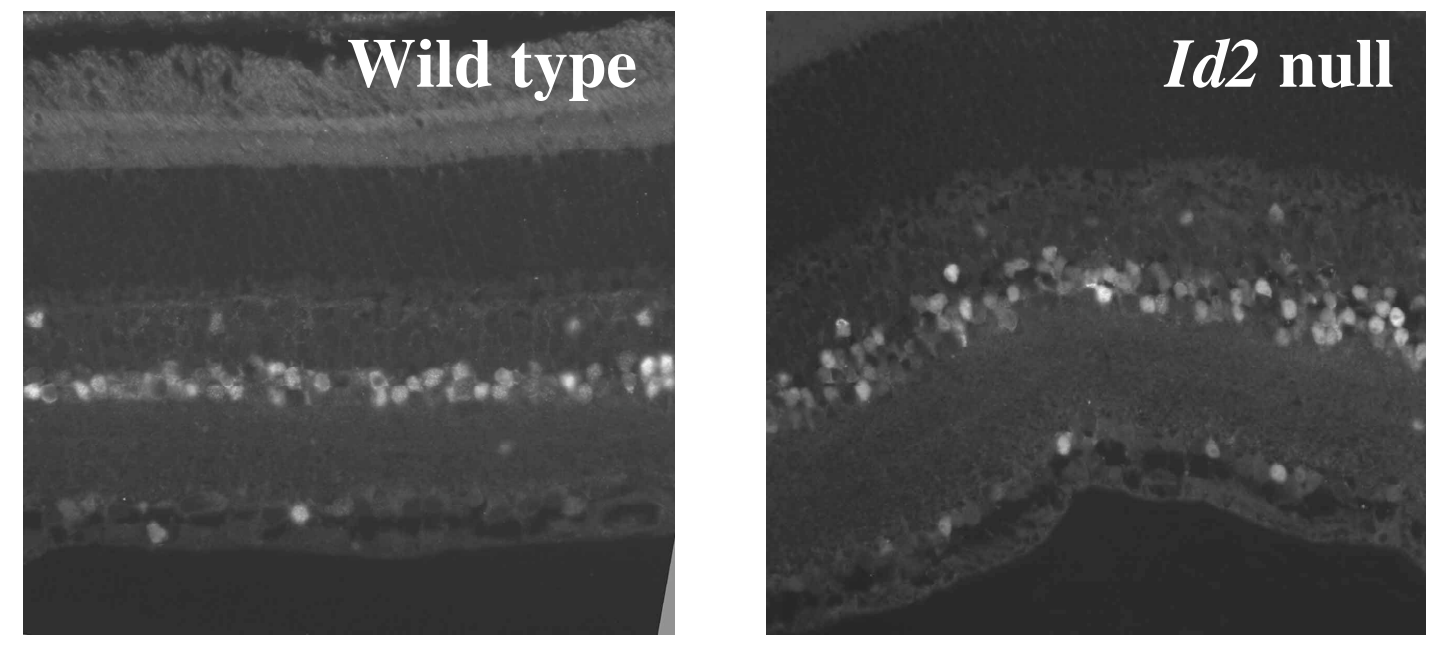

Figure D.1. ID2 antibody control using retina from Id2-null mice. 
Appendix E

Glossary 


\begin{tabular}{|c|c|}
\hline $\mathrm{A} 2 \mathrm{~m}$ & Alpha-2-macroglobulin \\
\hline Abi2 & abl-interactor 2 \\
\hline Agtpbp1* & ATP/GTP binding protein $1 *$ \\
\hline Aif1* & Alograft inflamatory factor $1 *$ \\
\hline Akap11 & A kinase anchor protein 11 \\
\hline Akap12 & A kinase anchor protein 12 \\
\hline Ania4 & Activity \& neurotransmitter-induced 4 \\
\hline Anxa5 & Annexin V \\
\hline Ap3m1 & Adaptor-related protein complex 3 , mu 1 \\
\hline Apobec1 & Apolipoprotein B editing protein \\
\hline Apoe & Apolipoprotein E \\
\hline Aprt & Adenine phosphoribosyltransferase \\
\hline Arhgap7 & Rho GTPase activating protein 7 \\
\hline Arl1 & ADP-ribosylation factor-like 1 \\
\hline Arl5 & ADP-ribosylationfactor-like5 \\
\hline Arpc1b & Actin-related protein complex $1 \mathrm{~b}$ \\
\hline Atf3 & Activating transcription factor 3 \\
\hline Bax & Bcl2-associated $\mathrm{X}$ protein \\
\hline $\mathrm{Bcl} 2$ & B-cell leukemia/lymphoma 2 \\
\hline Bfsp1 & Filensin \\
\hline Btg2 & B-cell translocation gene 2 \\
\hline $\mathrm{Clqb}$ & Complement component $1 \mathrm{qb}$ \\
\hline C1r & Complement component $1 \mathrm{r}^{*}$ \\
\hline C1s & Complement component $1 \mathrm{~s}$ \\
\hline $\mathrm{C} 3$ & Complement component 3 \\
\hline Camk2d & Ca calmodulin-dependent protein kinase $2 \mathrm{~d}$ \\
\hline Casp3 & Caspase 3 \\
\hline $\mathrm{Cbfb}$ & Core binding protein beta \\
\hline $\mathrm{Cd} 3612$ & Cd36-like 2 \\
\hline $\mathrm{Cd} 48$ & $\mathrm{Cd} 48$ \\
\hline $\mathrm{Cd} 74$ & $\mathrm{Cd} 74$ \\
\hline $\mathrm{Cd} 81$ & Cd81 (TAPA) \\
\hline CD82 & Cd82 (Kai1) \\
\hline $\mathrm{Cd} 9$ & $\mathrm{Cd} 9$ \\
\hline Cebpd & CCAAT/enhancer binding protein delta \\
\hline Cish3 & Cytokine inducible SH2-containing 3 \\
\hline $\mathrm{Clu}$ & Clusterin \\
\hline Cntf & Cntf \\
\hline Cntn1 & Contactin 1 \\
\hline Cox $7 \mathrm{a}$ & Cytochrome oxidase subunit VIIa \\
\hline $\mathrm{Cp}$ & Ceruloplasmin (ferroxidase) \\
\hline Crem & CAMP responsive element modulator \\
\hline Crot & Carnitine O-octanoyltransferase \\
\hline Cryaa & Crystallin alpha A \\
\hline Cryab & Crystallin alpha B \\
\hline Cryba1 & Crystallin beta A1/A3 \\
\hline Crybb2 & Crystallin beta B2 \\
\hline
\end{tabular}




\begin{tabular}{|c|c|}
\hline Crygd & Crystallin gamma D \\
\hline Cryge & Crystallin gamma-E \\
\hline Cts4 & Cystatin S \\
\hline Ctsc & Cathepsin C \\
\hline Ctsh & Cathepsin $\mathrm{H}$ \\
\hline Ctsl & Cathepsin L \\
\hline Ctss & Cathepsin S \\
\hline D123 & Cell cycle related D123 \\
\hline Dlg1 & Discs-large homolog 1 \\
\hline Dnajb9 & DnaJ (Hsp40) homolog, subfamily b9 \\
\hline Dpys14 & Dihydropyrimidinase-like 4 \\
\hline Drd5 & Dopamine receptor D5 \\
\hline Eef1a2 & Eukaryotic translation elongation factor $1 \mathrm{a} 2$ \\
\hline Egr1 & Early growth response 1 (Krox-24) \\
\hline Eif5 & Eukaryotic initiation factor 5 \\
\hline Fcgr3 & Fc receptor IgG, low affinity 3 \\
\hline Fos & c-fos \\
\hline Fos11 & Fos-like antigen 1 (Fra-1) \\
\hline Fuca & Fucosidase alpha-L-1 tissue \\
\hline Gfap & Gfap \\
\hline Gif & Intrinsic factor \\
\hline Gludins & Glucose-dependent insulinotropic \\
\hline Gp38 & Gycoprotein 38 \\
\hline Gq-alpha & Gq-alpha subunit \\
\hline Grifin & Galectin-related inter-fiber \\
\hline Gsta1 & Glutathione-S-transferase, alpha 1 \\
\hline Gtpi* & Interferon-g induced GTPase* \\
\hline Gucy $2 \mathrm{e}$ & Guanylyl cyclase $2 \mathrm{e}$ \\
\hline Hes3 & hairy and enhancer of split 3 (Drosophila) \\
\hline Hsd17b4 & Hydroxysteroid 17-b dehydrogenase 4 \\
\hline $\mathrm{Id} 2$ & inhibitor of DNA binding 2 \\
\hline $\operatorname{Idh} 2$ & Isocitrate dehydrogenase 2 \\
\hline Igf1 & Insulin-like growth factor 1 \\
\hline Igfals & IGF binding protein complex acid-labile \\
\hline $\mathrm{Il} 1 \mathrm{~b}$ & Interleukin 1 , beta \\
\hline Il6r & Interleukin 6 receptor \\
\hline Impa1 & Inositol Monophosphatase \\
\hline IMPG2 & Proteoglycan 10.2 \\
\hline Inpp4 & Inositol polyphosphate 4-phosphatase IIa \\
\hline Insig1 & Growth response protein CL-6 \\
\hline Irf1 & Interferon regulatory factor 1 \\
\hline Itgb1 & Integrin, beta 1 \\
\hline Jag1 & Jagged 1 \\
\hline Kcnj & Potassium inwardly-rectifying channel \\
\hline Krt1-14* & Keratin complex-1, acidic, gene $14^{*}$ \\
\hline Lamp2 & Lysosomal membrane glycoprotein 2 \\
\hline $\operatorname{Lcn} 2$ & Lipocalin 2 \\
\hline
\end{tabular}




\begin{tabular}{|c|c|}
\hline Lhx2 & LIM homeobox protein 2 \\
\hline $\operatorname{Lim} 2$ & Lens intrinsic membrane protein $2(19 \mathrm{kDa})$ \\
\hline Lip1 & Lysosomal acid lipase \\
\hline Litaf * & LPS-induced TNF-alpha factor* \\
\hline Lman1 & Lectin mannose-binding 1 \\
\hline Lyz & Lysozyme \\
\hline Lyzs & lysozyme \\
\hline Mak & Male germ cell-associated kinase \\
\hline Mat2a & Methionine adenosyltransferase IIa \\
\hline Mgp & Matrix Gla protein \\
\hline Mllt4 & Afadin \\
\hline Mt1a & Metallothionein \\
\hline Mx3 & Myxovirus resistance 3 \\
\hline Nap111 & Nucleosome assembly protein 1-like 1 \\
\hline Ndufa6* & NADH dehydrogenase $1 \mathrm{a} 6 *$ \\
\hline Neurod1 & neurogenic differentiation 1 \\
\hline Neurod2 & neurogenic differentiation 2 \\
\hline Nfib & nuclear factor I/B \\
\hline Nfkb1 & Nuclear factor kappa B p105 \\
\hline $\mathrm{Nr} 4 \mathrm{a} 1$ & nuclear receptor subfamily 4 , group A, member 1 \\
\hline $\mathrm{Nr} 4 \mathrm{a} 1$ & Nuclear receptor subfamily $4 \mathrm{a} 1$ \\
\hline Nup153 & Nucleoporin $153 \mathrm{kD}$ \\
\hline P2ry4 & Pyrimidinergic receptor P2Y 4 \\
\hline P4hb & Prolyl 4-hydroxylase beta \\
\hline p65 & p65 protein \\
\hline Pak3 & p21 CDKN1A activated kinase 3 \\
\hline Pax6 & paired box gene 6 \\
\hline PDE4 & cAMP phosphodiesterase (PDE4) \\
\hline Pde4b & Phosphodiesterase 4B cAMP-specific \\
\hline PDP1 & Pyruvate dehydrogenase phosphatase 1 \\
\hline Pex11a & Peroxisomal biogenesis factor $11 \mathrm{~A}$ \\
\hline Pik3r3 & Phosphatidylinositol 3-kinase p55 \\
\hline PLA2IR & Phospholipase A2 receptor \\
\hline Plat & Plasminogen activator tissue \\
\hline Plrg1 & Pleiotropic regulator 1 \\
\hline Pmp22 & Peripheral myelin protein 22 \\
\hline Pou2f1 & POU domain, class 2 , transcription factor 1 \\
\hline Ppap2b & Phosphatidic acid phosphatase type 2B \\
\hline Ppicap & Peptidylprolyl isomerase C-associated \\
\hline Ppp2rb & Phosphatase 2, regulatory subunit B \\
\hline Prkacb & cAMP-dependent protein kinase, beta \\
\hline Prkr & ElF2alpha kinase, interferon-inducible \\
\hline Pros 1 & Protein $\mathrm{S}$ \\
\hline Ptgfr & Prostaglandin F receptor \\
\hline Ptn & Pleiotrophin \\
\hline Qpct & Glutaminyl cyclase \\
\hline Rnf4 & Ring finger protein 4 \\
\hline
\end{tabular}




$\begin{array}{ll}\text { Robo1 } & \text { roundabout homolog 1 (Drosophila) } \\ \text { Rpo1-1 } & \text { RNA polymerase 1-1 } \\ \text { RT1.Da u } & \text { MHC class IIa RT1.u-D-alpha } \\ \text { RT1Aw2 } & \text { MHC class Ib alpha (RT1Aw2) } \\ \text { RT1-Mb } & \text { MHC class II DM beta } \\ \text { RT1-S3 } & \text { MHC class Ib (RT1-S3) } \\ \text { RT-BM1 } & \text { MHC class Ib BM1k antigen } \\ \text { Rtn4 } & \text { reticulon 4 } \\ \text { Scyb10 } & \text { Small inducible cytokine B10 } \\ \text { Scyb13 } * & \text { Small inducible cytokine B13* } \\ \text { Slc1a4 } & \text { Solute carrier family 1, member 4 } \\ \text { Slc25a20 } & \text { Solute carrier family 25, member 20 } \\ \text { Slc2a3 } & \text { Solute carrier family 2, member 3 } \\ \text { Snca } & \text { Synuclein 1 } \\ \text { Snn } & \text { Stannin } \\ \text { Sparc } & \text { Sparc } \\ \text { Spin2c } & \text { Serine protease inhibitor } \\ \text { Ssb } & \text { Sjogren syndrome antigen B } \\ \text { Stat3 } & \text { Stat3 } \\ \text { Stc1 } & \text { Stanniocalcin 1 } \\ \text { Sv2b } & \text { Synaptic vesicle glycoprotein 2b } \\ \text { Symbol } & \text { Name } \\ \text { Tcf4 } & \text { transcription factor 4 } \\ \text { Tpm3 } & \text { Tropomyosin 3 gamma } \\ \text { Trib3 } & \text { tribbles homolog 3 (Drosophila) } \\ \text { Ttgn1 } & \text { Trans-golgi network protein 1 } \\ \text { Tubb1 } & \text { Tubulin, beta 5 } \\ \text { UGT1A7 } & \text { UDP-glucuronosyltransferase 1A7 } \\ \text { unr } & \text { Unr protein } \\ \text { Vcam1 } & \text { Vcam 1 } \\ \text { Vhl } & \text { Von Hippel-Lindau syndrome } \\ \text { Ywhae } & \text { Tyrosine 3/tryptophan 5-monooxygenase E } \\ \text { Ywhaq } & \text { Tyrosine 3/tryptophan 5-monooxygenase theta } \\ \text { Zfp36l1 } & \text { Zinc finger protein 36, C3H type-like 1 } \\ & \end{array}$




\section{Vita}

\section{EDUCATION:}

Doctor of Philosophy, Expected graduation date, May 2006

University of Tennessee, Memphis, Tennessee

Departments: Anatomy \& Neurobiology, and Ophthalmology

Passed Defense: December 1, 2005

Thesis: Genetic networks controlling retinal injury

Bachelor of Science, Biology 1998, Christian Brothers University, Memphis, Tennessee

Bachelor of Arts, Economics 1996, Rhodes College, Memphis, Tennessee

Honors:

- Cum Laude Graduate

- Alpha Chi (Scholarship)

- First Year Calculus Award, May 1993

\section{AWARDS:}

Travel Award, University of Tennessee, 2005

Fight For Sight Summer Student Fellowship. Summer 2004

Tennessee Academy of Sciences Collegiate Division, Research presentation, $3^{\text {rd }}$ place, 1998

\section{RESEARCH BACKGROUND:}

Glia cell proliferation and reactivity

Microarray analysis of retinal and CNS wound healing

RNA interference as a tool to modulate reactive gliosis in culture

\section{POST-DOCTORAL RESEARCH INTERESTS:}

Role of glia cells during retinal development and disease

Transcriptional regulation of reactive and developmental retinal genes

Molecular and genetic approaches to treat retinal conditions: RNA interference, gene therapy, and cell transplantation.

\section{PUBLICATIONS:}

Vázquez-Chona FR, Wang XD, Wang XF, Geisert EE Jr. Genetic influences on gene expression and retinal wound healing. Manuscript in preparation.

Vázquez-Chona FR, Khan AN, Chan CK, Moore AN, Dash, PK, Hernandez MR, Lu L, Chesler EJ, Manly KF, Williams RW, Geisert EE Jr. Genetic networks controlling retinal injury. Mol Vis. 11:958-70.

Vázquez-Chona F, Song BK, Geisert EE Jr. Temporal Changes in Gene Expression After Injury in the Rat Retina. Invest Opthal Vis Sci, 2004;45:2737-46.

Song BK, Geisert GR, Vázquez-Chona F, Geisert EE Jr. Temporal Regulation of CD81 following retinal injury in the rat. Neuro Lett. 2003;20:29-32.

Vázquez-Chona F, Geisert EE Jr. N-cadherin at the glial scar in the rat. Brain Res. 1999;838:45-50. 


\section{TEACHING EXPERIENCE:}

Dental Histology Lab Assistant. University of Tennessee, Memphis, Tennessee, Fall 2002

Biology, Anatomy \& Physiology, and Physics Instructor. Memphis Catholic High School, Memphis,

Tennessee, Fall 1998 - Spring 2000

\section{ABSTRACTS:}

Vázquez-Chona FR, Geisert EE Jr. Genetic networks controlling retinal injury. Ocular cell and Molecular Biology meeting, 2005

Vázquez-Chona FR, Geisert EE Jr. Genetic networks controlling retinal injury. Neuroscience Meeting, 2005

Vázquez-Chona F, Khan AN, Geisert EE Jr. Transcription regulator ID2 controls glia cell reactivity. Neuroscience Meeting, 271.1, 2004

Geisert EE, Khan AN, Lu L, Chesler EJ, Manly KF, Williams RW, Vázquez-Chona F. Master Regulators of Glia Cell Reactivity. Neuroscience Meeting, 271.2, 2004

Vázquez-Chona F, Lu L, Chesler EJ, Williams RW, Geisert EE Jr. Common gene expression and regulation following injury in the retina, brain, and spinal cord. ARVO, 2004

Geisert EE Jr, Vázquez-Chona F, Lu L, Chesler EJ, Williams RW. The control of gene expression following injury in the rat retina: QTL and PCA. ARVO, 2003.

Vázquez-Chona F, Geisert EE Jr, Geisert G. Changes in gene expression following injury in the retina. Neuroscience Meeting, 2002

Geisert EE Jr, Vázquez-Chona F, Geisert G. Tetraspanins in rat retina injury. ARVO, 2002

Vázquez-Chona F, Geisert EE Jr, Geisert G. Changes in gene expression following injury in the retina. Spring Brain Conference, 2002

Vázquez-Chona F, Geisert EE Jr. Differential gene expression in the developing and injured mouse visual cortex. Spring Brain Conference, 2001

\section{SCHOLARLY PRESENTATIONS:}

$N$-cadherin at the glial scar in the rat. Presented at the Alpha Chi regional meeting in Montgomery, Alabama, April 1998

$\mathrm{N}$-cadherin at the glial scar in the rat. Presented at the Tennessee Academy of Sciences Collegiate Division Research, Jackson, Tennessee, March 1998

\section{LAB TECHNIQUE EXPERIENCE:}

Molecular. RNA interference, cDNA \& Affymetrix microarrays, qRT-PCR

Histology \& protein methods. Immunohistochemistry, western blotting, ELISA, and flow cytometry

Cell culture. Harvesting and maintenance of retinal explants and cortical astrocytes

Basic mouse husbandry.

Surgery. Retinal tears, vitreal injections, optic nerve crush, rat cortical stabs

\section{PERSONAL INFORMATION:}

Place and Date of Birth. Mexico City, 1974

Marital Status: Married_December 2004 — to Sara Vazquez, Pharmacy Resident, June '05 - June '06 Immigration Status: Applied for U.S. permanent residency January 2005. 